University of South Florida

DIGITAL COMMONS

@ UNIVERSITY OF SOUTH FLORIDA
Digital Commons @ University of

South Florida

$10-2000$

\title{
1999 Transit Customer Satisfaction Index for Florida Transit Properties
}

CUTR

Follow this and additional works at: https://digitalcommons.usf.edu/cutr_nctr

\section{Scholar Commons Citation}

CUTR, "1999 Transit Customer Satisfaction Index for Florida Transit Properties" (2000). Research Reports.

2.

https://digitalcommons.usf.edu/cutr_nctr/2

This Technical Report is brought to you for free and open access by the National Center for Transit Research (NCTR) Archive (2000-2020) at Digital Commons @ University of South Florida. It has been accepted for inclusion in Research Reports by an authorized administrator of Digital Commons @ University of South Florida. For more information, please contact digitalcommons@usf.edu. 


\title{
1999 TRANSIT CUSTOMER SATISFACTION INDEX
}

\author{
Final Report \\ Results of Survey and Conclusions
}

\author{
Prepared for: \\ Department of Transportation \\ State of Florida \\ By: \\ Center for Urban Transportation Research \\ College of Engineering \\ University of South Florida
}

October 2000 

Florida Department of Transportation

605 Suwannee Street

Tallahassee, Florida 32399-0450

(904) 488-7774

Fax (904) 922-4942

Project Manager: Jon Ausman

\section{Center for Urban Transportation Research}

University of South Florida

4202 E. Fowler Avenue, CUT 100

Tampa, Florida 33620-5350

(813) 974-3120

Suncom 574-3120

Fax (813) 974-5168

Principal Investigators: $\quad$ Francis Cleland

Brenda Thompson

The opinions, findings and conclusions expressed in this publication are those of the authors and not necessarily those of the State of Florida Department of Transportation.

Prepared in cooperation with the State of Florida Department of Transportation. 


\section{TABLE OF CONTENTS}

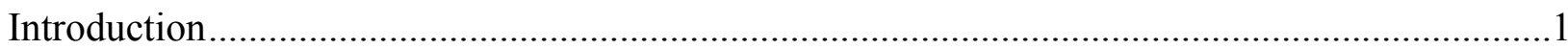

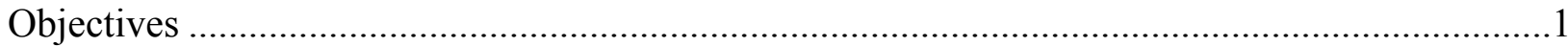

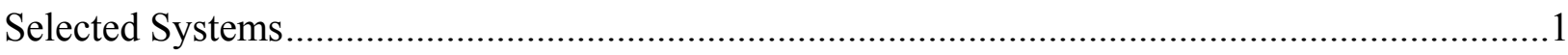

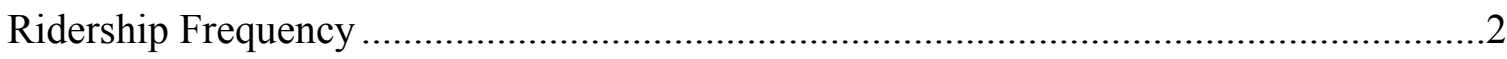

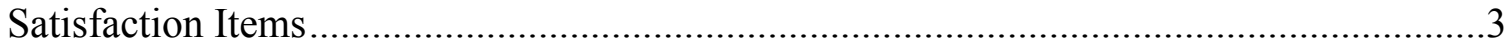

Handling of the Ridership Frequency Response Bias..............................................

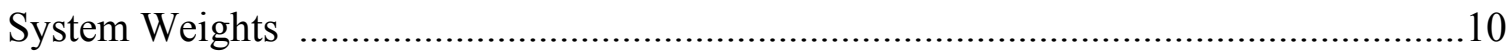

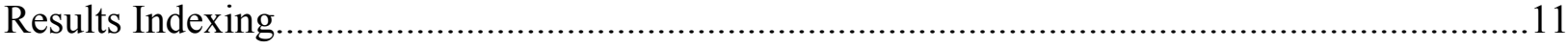

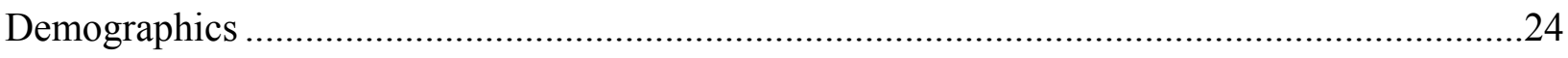

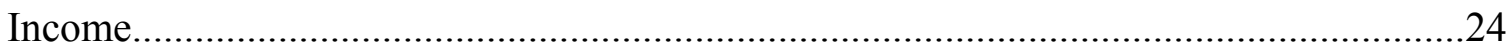

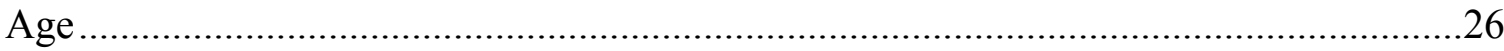

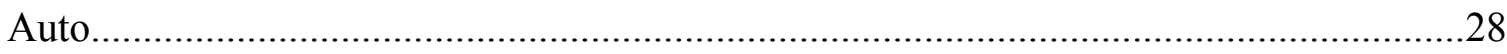

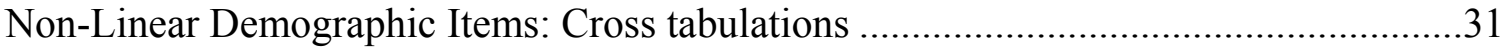

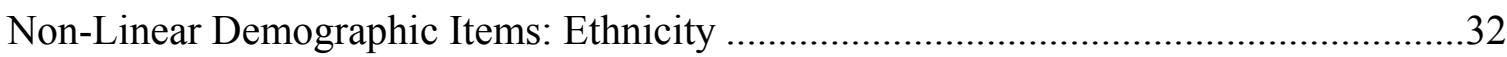

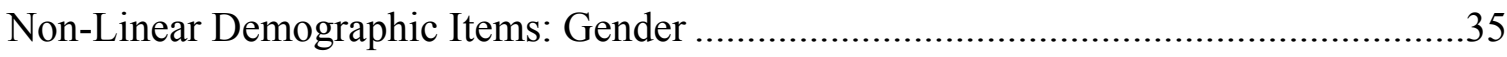

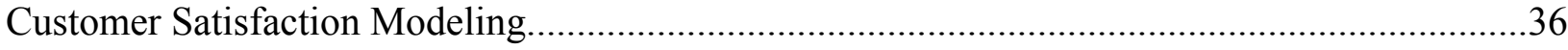

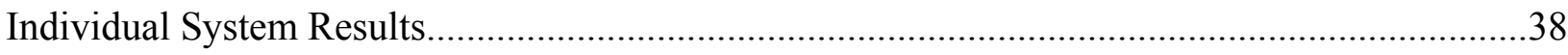

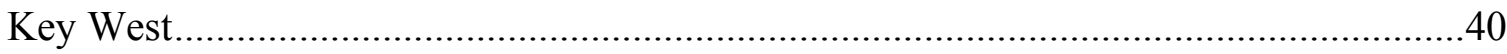

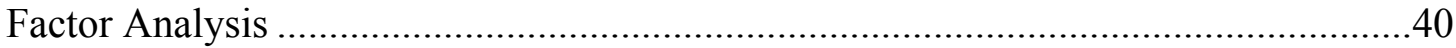

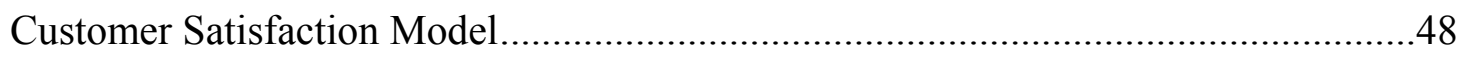

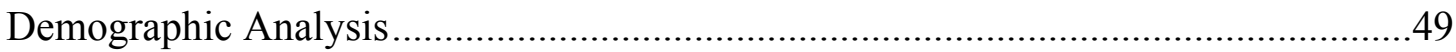

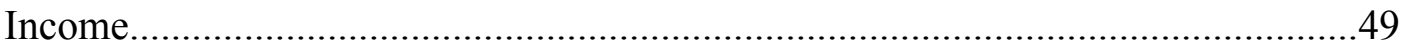

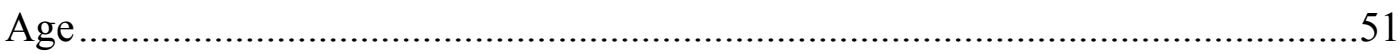

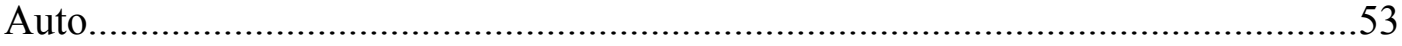

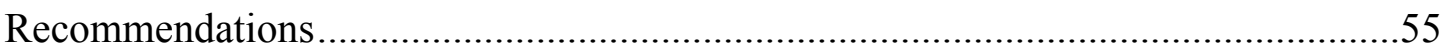

JTA

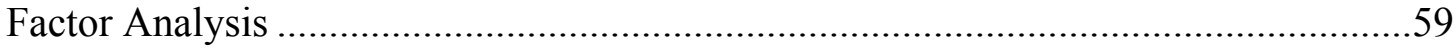

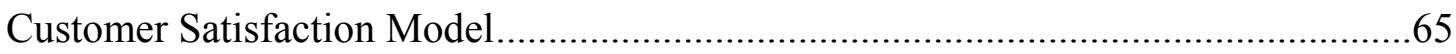

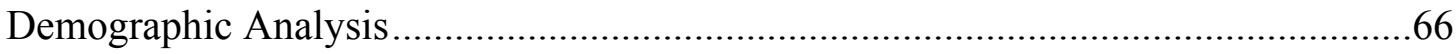

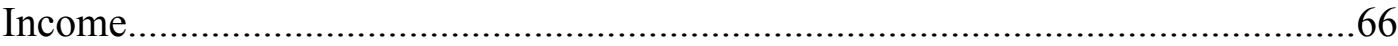

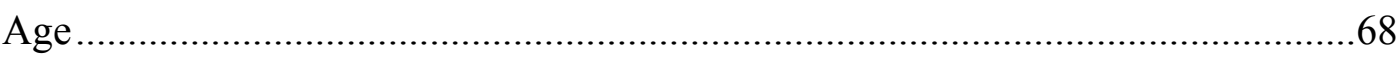

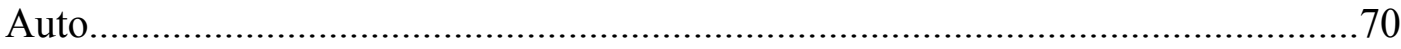

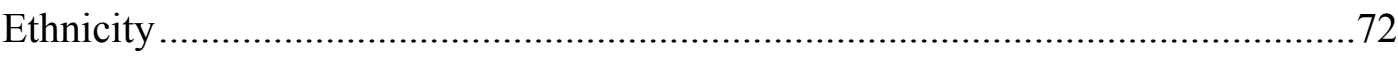

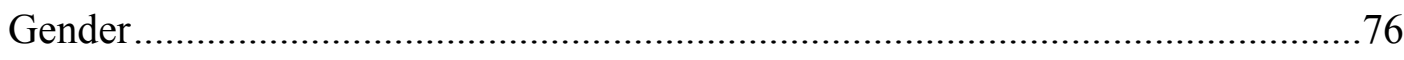

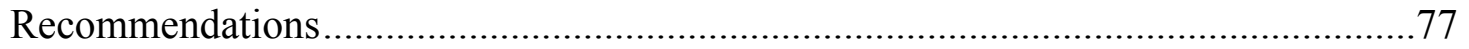




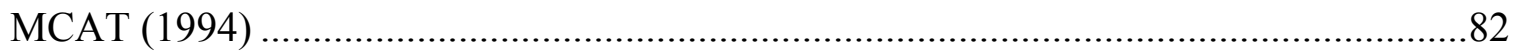

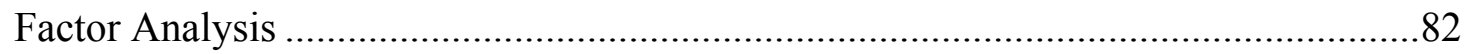

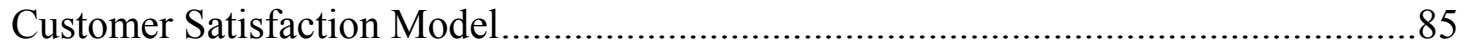

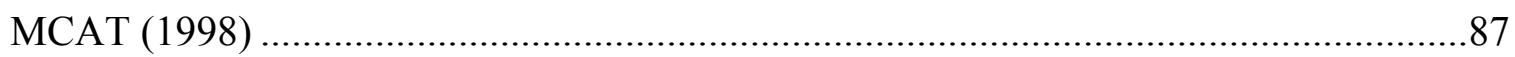

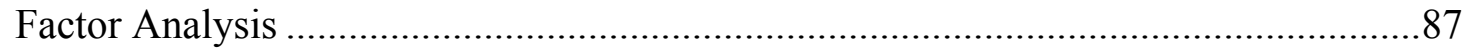

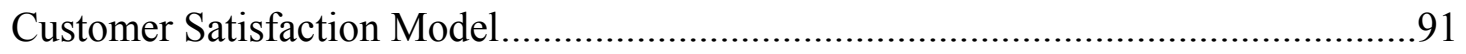

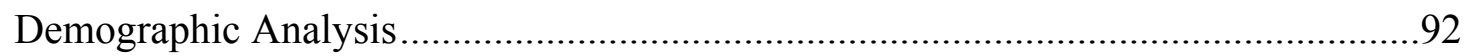

Income

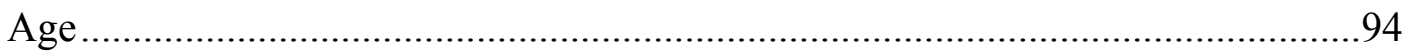

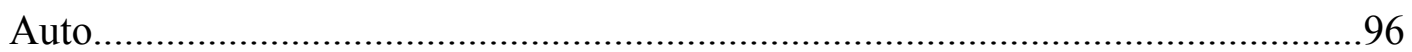

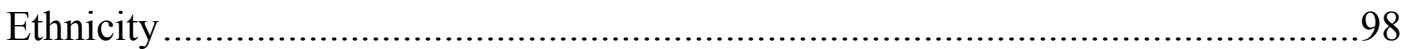

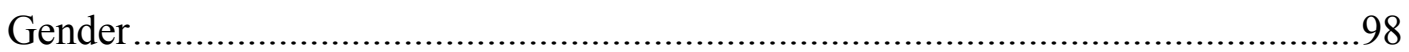

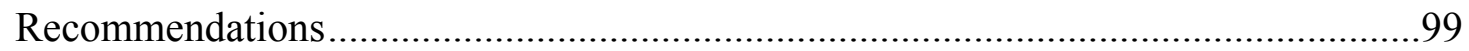

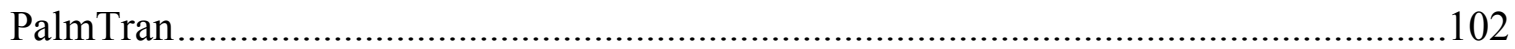

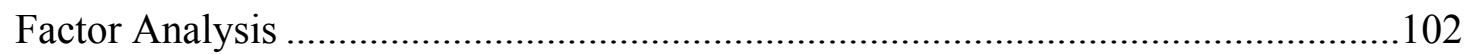

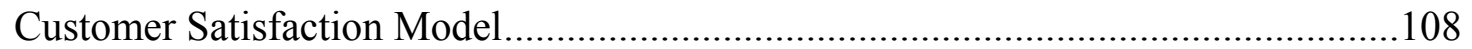

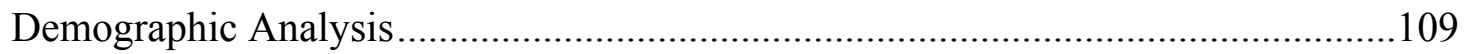

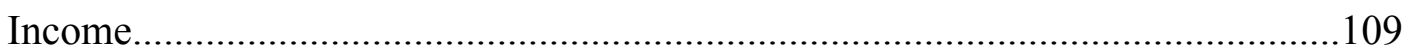

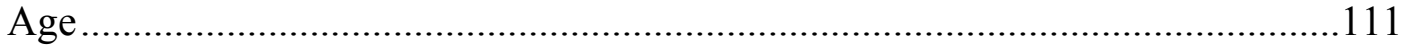

Auto

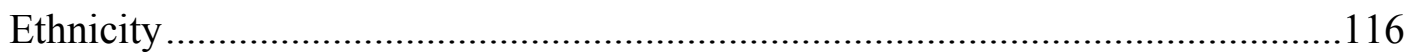

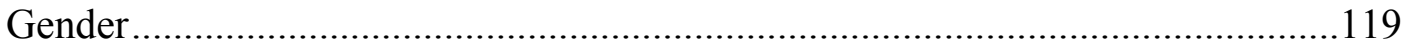

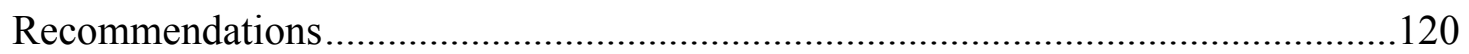

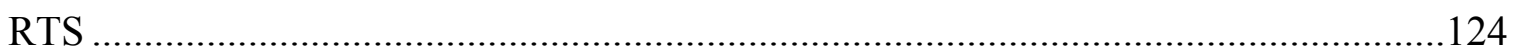

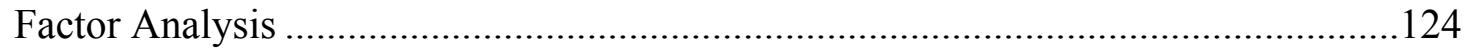

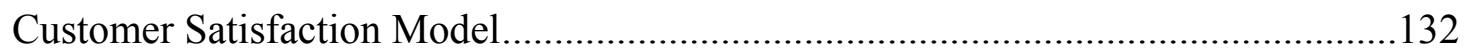

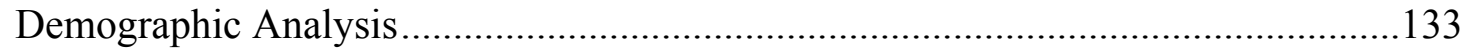

Income

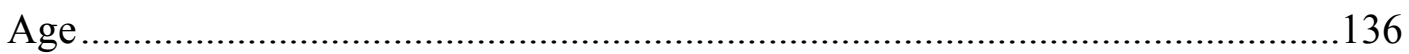

Auto

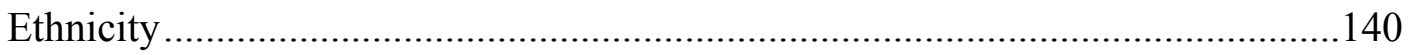

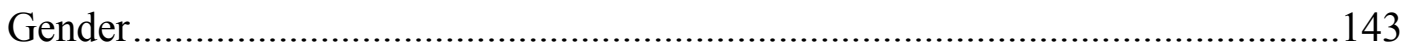

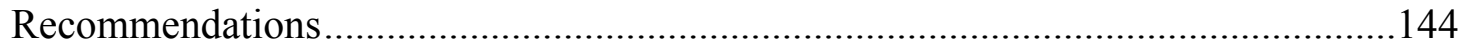

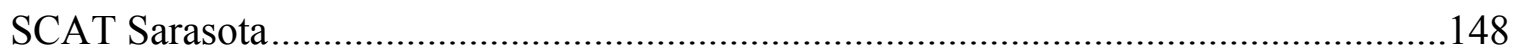

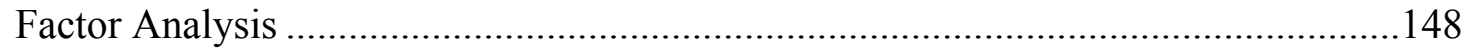

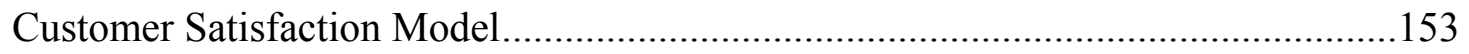

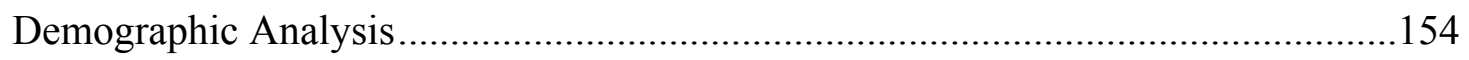




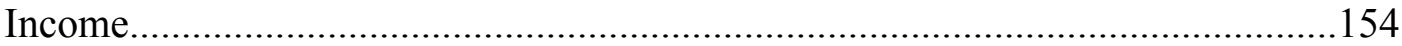

Age

Auto

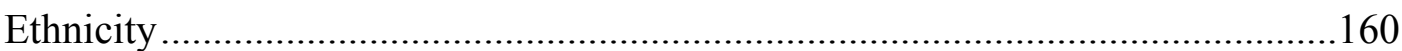

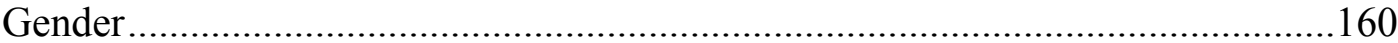

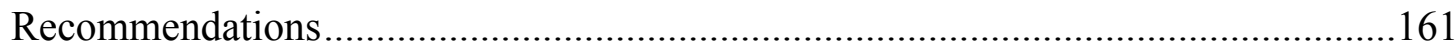

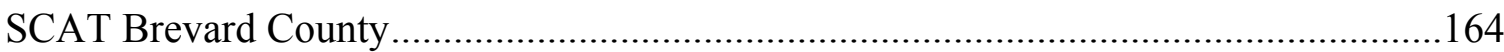

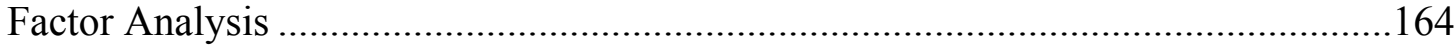

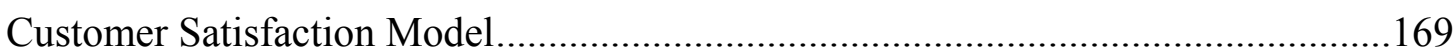

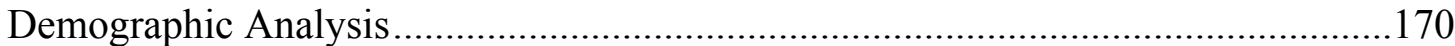

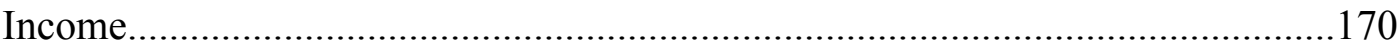

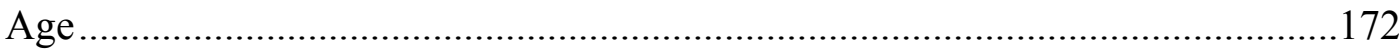

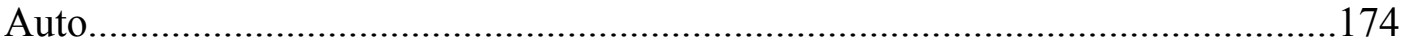

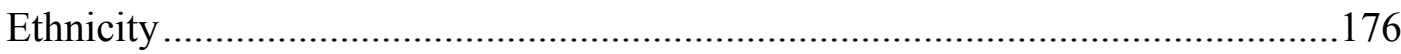

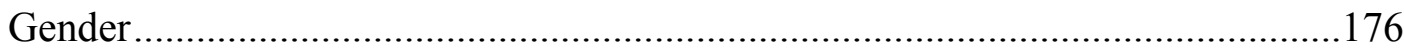

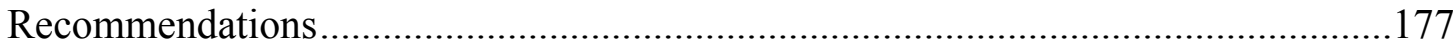

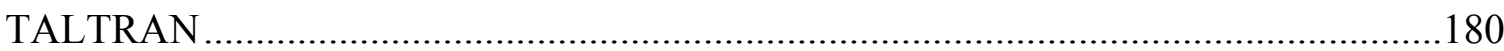

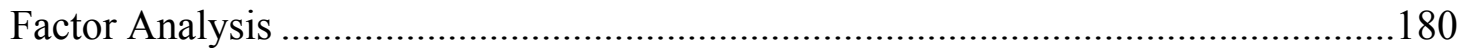

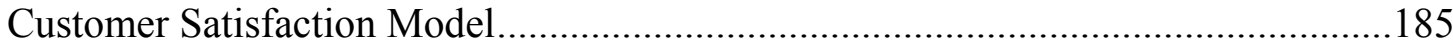

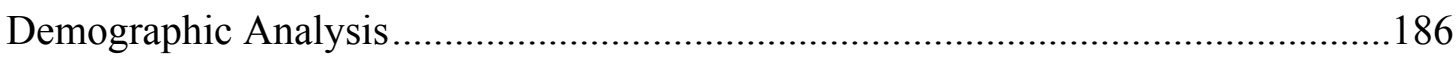

Income

Age

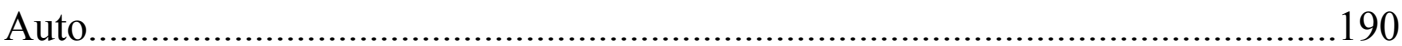

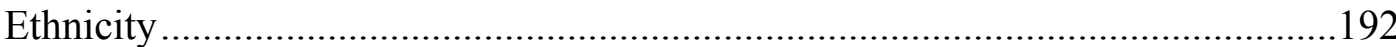

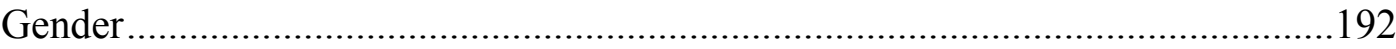

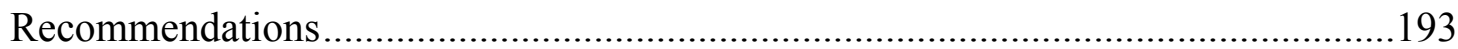

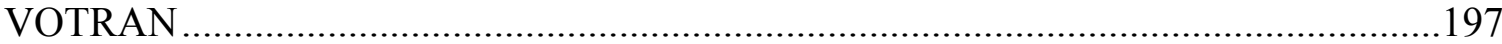

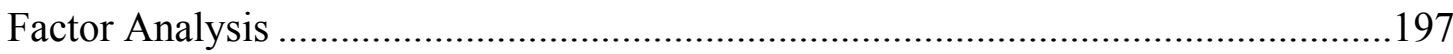

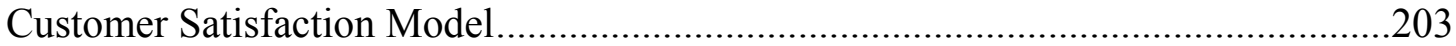

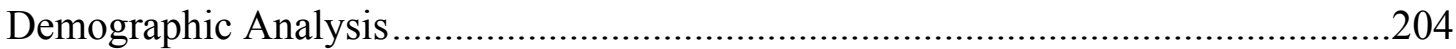

Income

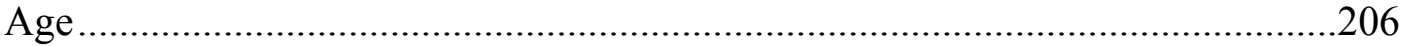

Auto

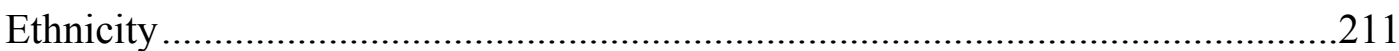

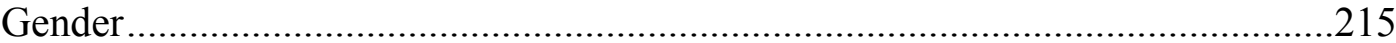

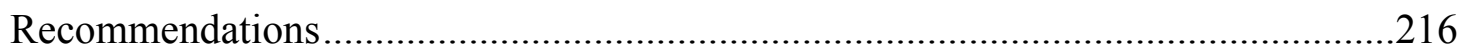




\section{List of Tables}

Table 1: On-Board Questionnaire Item Matrix......................................................................4

Table 2: Relationship of Rider Use Frequency to Percentage of Trips Taken ............................9

Table 3: 1999 Overall Transit Customer Satisfaction Index Summary .......................................12

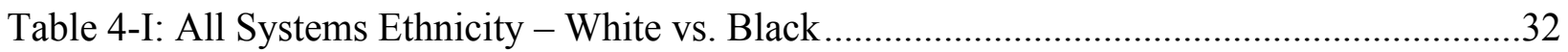

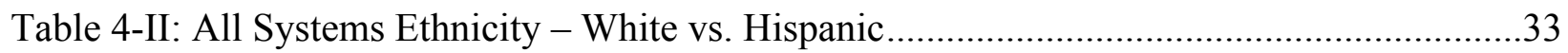

Table 4-III: All Systems Ethnicity - Black vs. Hispanic .........................................................33

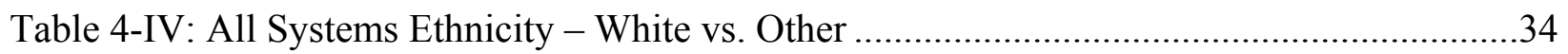

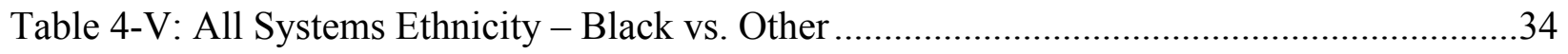

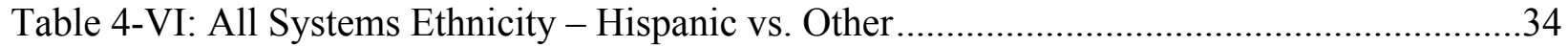

Table 5-I: Key West Factor 1 - System Design ........................................................................40

Table 5-II: Key West Factor 2 - Span of Service..................................................................41

Table 5-III: Key West Factor 3 - Perceptions of Safety........................................................42

Table 5-IV: Key West Factor 4 - Transfers.........................................................................43

Table 5-V: Key West Factor 5 - Value ....................................................................................44

Table 5-VI: Key West Factor 6 - Comfort of Ride ................................................................45

Table 5-VII: Key West Factor 7 - Schedule..........................................................................46

Table 5-VIII: Key West Factor 12 - Cleanliness/Safety .....................................................47

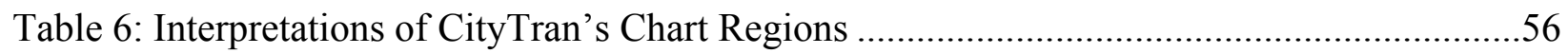

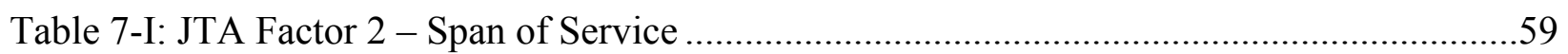

Table 7-II: JTA Factor 3 - Perceptions of Safety ….........................................................6

Table 7-III: JTA Factor 4 - Transfers ....................................................................................61

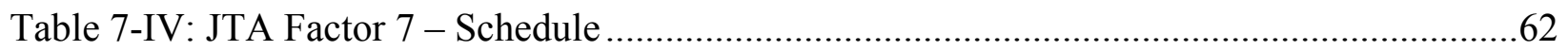

Table 7-V: JTA Factor 8 - Value/Timeliness of Service ......................................................63

Table 7-VI: JTA Factor 9 - Experience of the Bus Ride.........................................................64

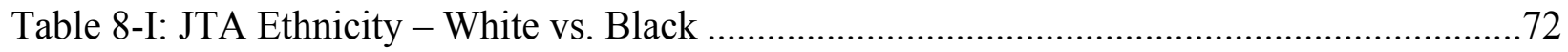

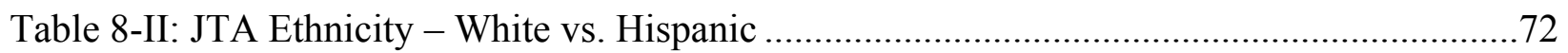

Table 8-III: JTA Ethnicity - Black vs. Hispanic ..................................................................73

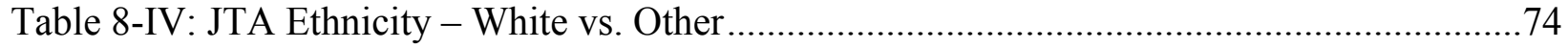

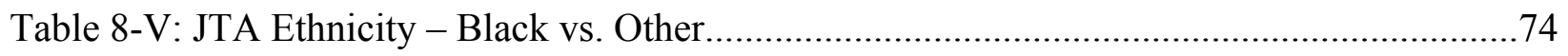

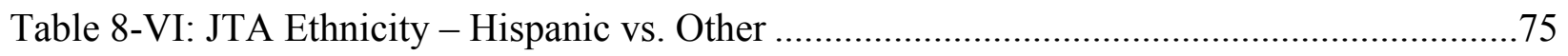

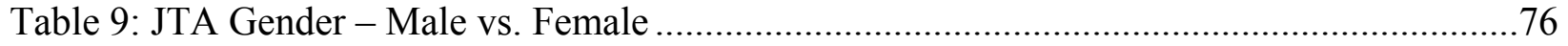

Table 10: Interpretations of JTA's Chart Regions ............................................................... 78

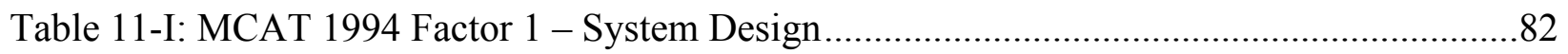

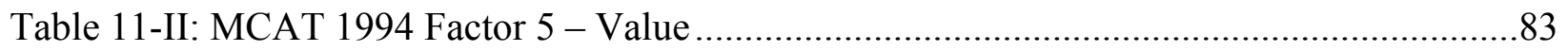

Table 11-III: MCAT 1994 Factor 9 - Experience of the Bus Ride ............................................83 
Table 11-IV: MCAT 1994 Factor 10 - Timeliness of Service ...................................................84

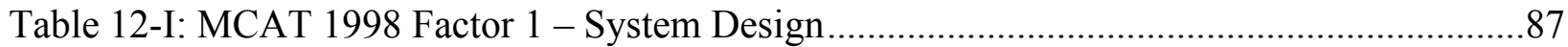

Table 12-II: MCAT 1998 Factor 7 - Printed Schedules.............................................................8

Table 12-III: MCAT 1998 Factor 8 - Value/Timeliness ........................................................89

Table 12-IV: MCAT 1998 Factor 9 - Experience of the Bus Ride ............................................90

Table 13: MCAT 1998 Ethnicity - White vs. Black ...............................................................98

Table 14: MCAT 1998 Gender - Male vs. Female ...................................................................98

Table 15: Interpretations of MCAT's Chart Regions ..........................................................100

Table 16-I: PalmTran Factor 1 - System Design.....................................................................102

Table 16-II: PalmTran Factor 2 - Span of Service .................................................................103

Table 16-III: PalmTran Factor 3 - Perceptions of Safety ........................................................104

Table 16-IV: PalmTran Factor 5 - Value ........................................................................ 105

Table 16-V: PalmTran Factor 6 - Comfort of Ride..............................................................106

Table 16-VI: PalmTran Factor 7 - Schedule ........................................................................106

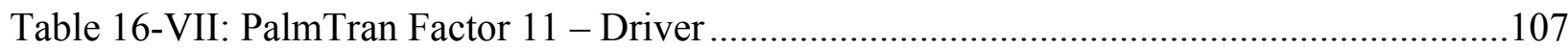

Table 17-I: PalmTran Ethnicity - White vs. Black...............................................................115

Table 17-II: PalmTran Ethnicity - White vs. Hispanic ......................................................116

Table 17-III: PalmTran Ethnicity - Black vs. Hispanic ..........................................................116

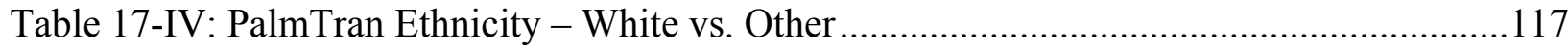

Table 17-V: PalmTran Ethnicity - Black vs. Other.............................................................. 117

Table 17-VI: PalmTran Ethnicity - Hispanic vs. Other .....................................................118

Table 18: PalmTran Gender - Male vs. Female ...................................................................119

Table 19: Interpretations of PalmTran's Chart Regions .......................................................121

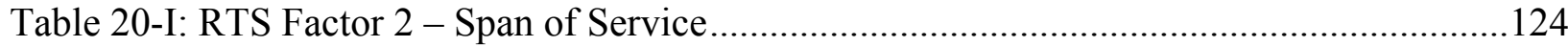

Table 20-II: RTS Factor 3 - Perceptions of Safety...............................................................125

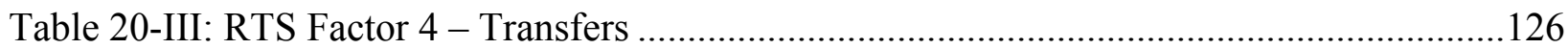

Table 20-IV: RTS Factor 5 - Value...................................................................................127

Table 20-V: RTS Factor 6 - Comfort of Ride .....................................................................128

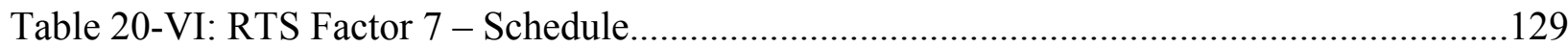

Table 20-VII: RTS Factor 10 - Timeliness of Service ............................................................130

Table 20-VIII: RTS Factor 11 - Driver .................................................................................131

Table 21-I: RTS Ethnicity - White vs. Black ........................................................................ 140

Table 21-II: RTS Ethnicity - White vs. Hispanic ................................................................ 141

Table 21-III: RTS Ethnicity - Black vs. Hispanic .........................................................141

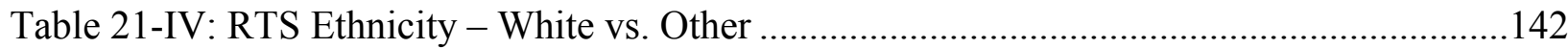

Table 21-V: RTS Ethnicity - Black vs. Other ..................................................................142

Table 21-VI: RTS Ethnicity - Hispanic vs. Other .................................................................142

Table 22: RTS Gender - Male vs. Female........................................................................143 
Table 23: Interpretations of RTS Chart Regions 145

Table 24-I: SCAT Sarasota Factor 1 - System Design.............................................................148

Table 24-II: SCAT Sarasota Factor 2 - Span of Service ..........................................................149

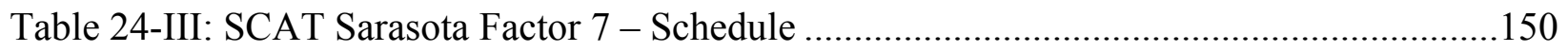

Table 24-IV: SCAT Sarasota Factor 8 - Value/Timeliness.......................................................151

Table 24-V: SCAT Sarasota Factor 9 - Experience of the Bus Ride ……………………….......152

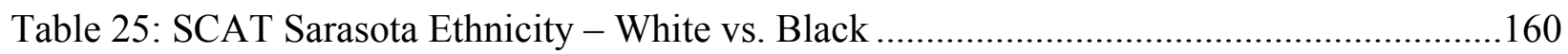

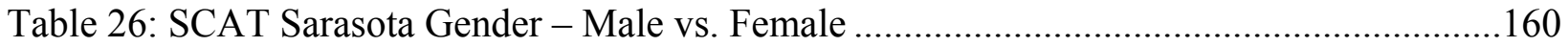

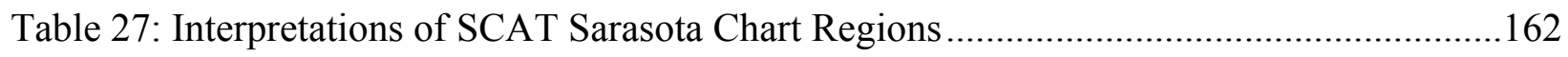

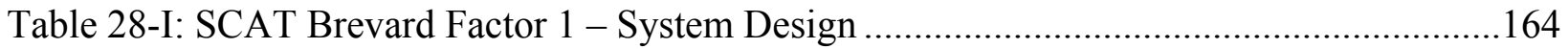

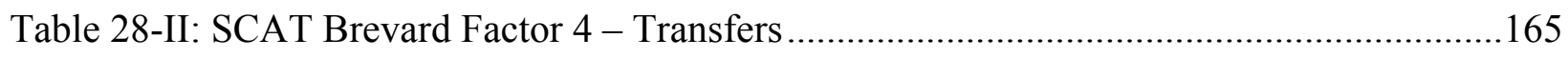

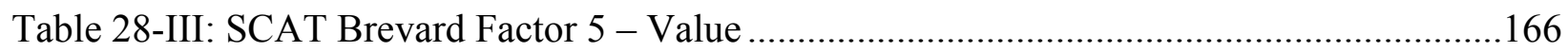

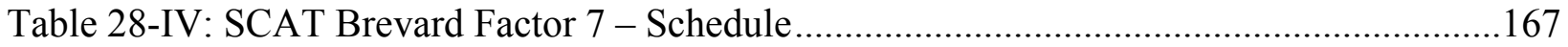

Table 28-V: SCAT Brevard Factor 9 - Experience of the Bus Ride...........................................168

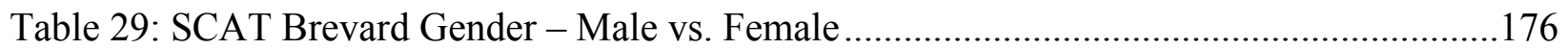

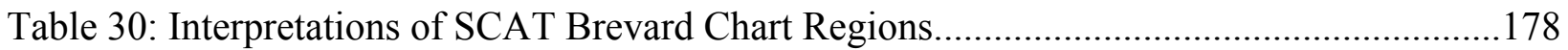

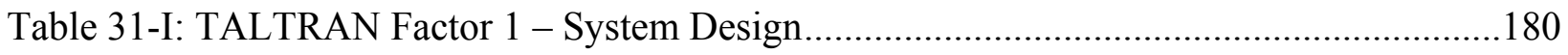

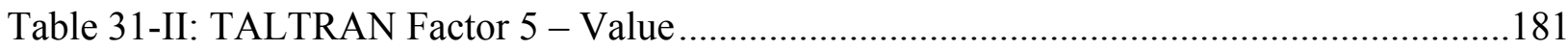

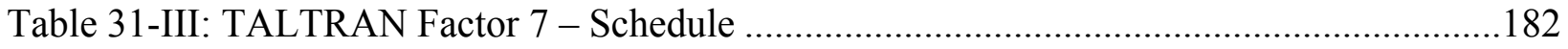

Table 31-IV: TALTRAN Factor 7 - Experience of the Bus Ride ...........................................183

Table 31-V: TALTRAN Factor 10 - Timeliness of Service .....................................................184

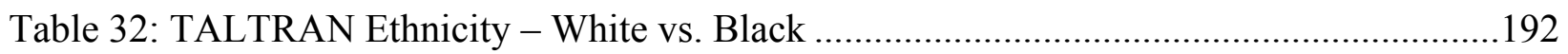

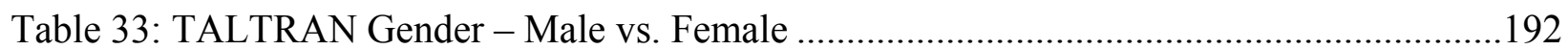

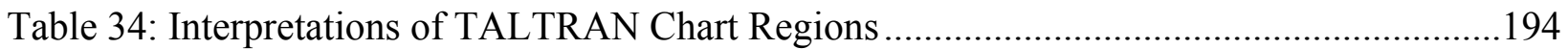

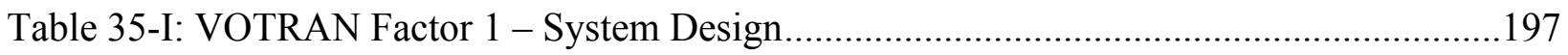

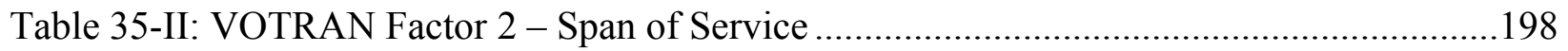

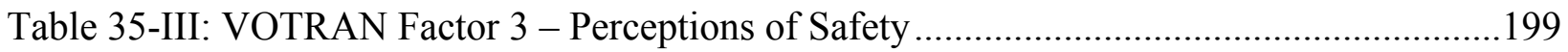

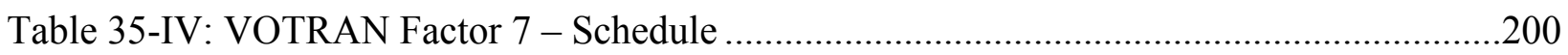

Table 35-V: VOTRAN Factor 9 - Experience of the Bus Ride ………………………...............201

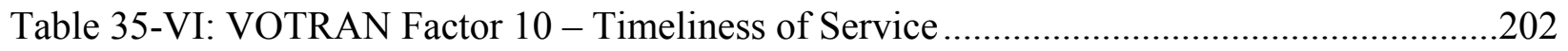

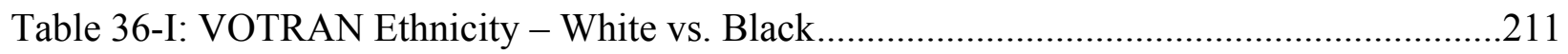

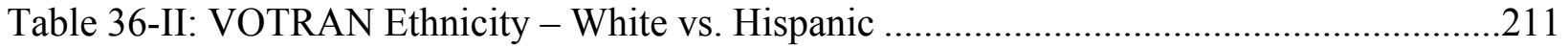

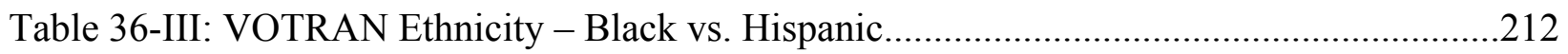

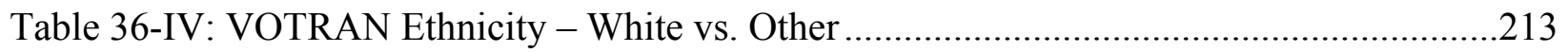

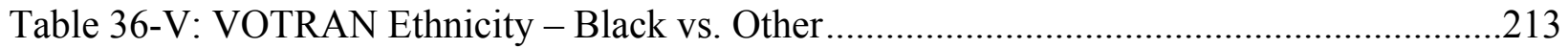

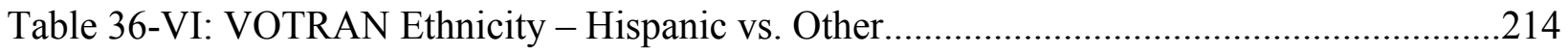

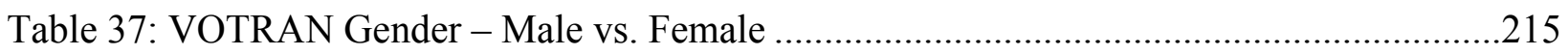

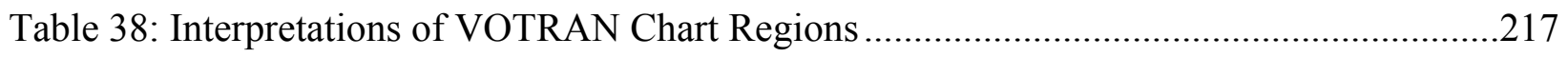


List of Figures

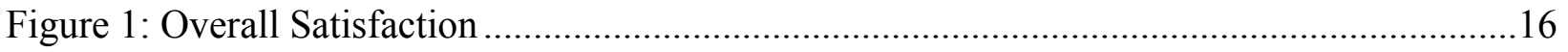

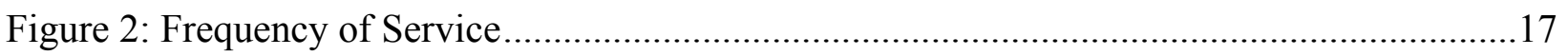

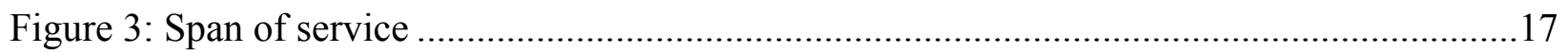

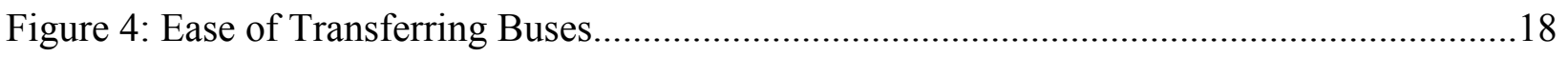

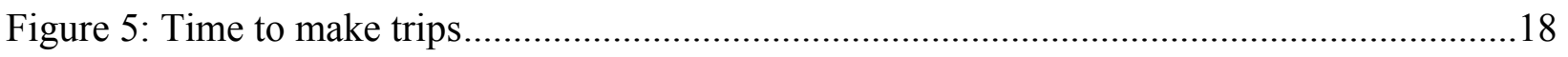

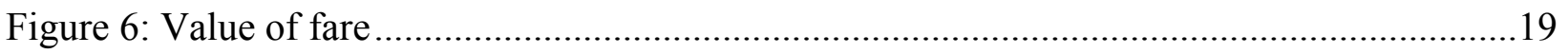

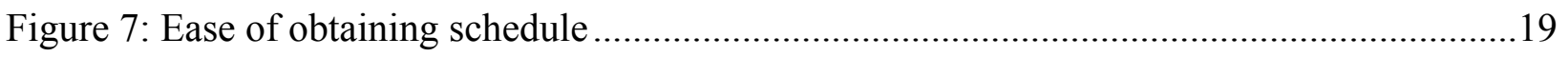

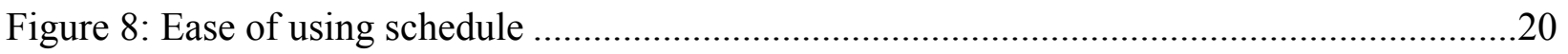

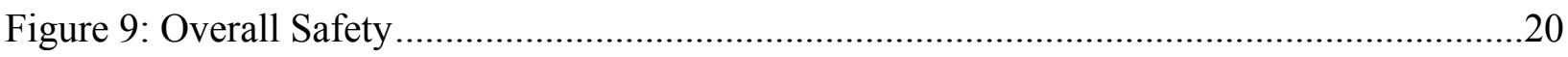

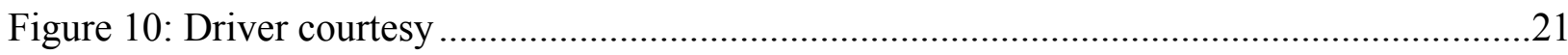

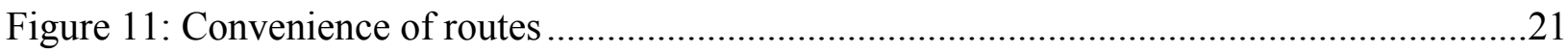

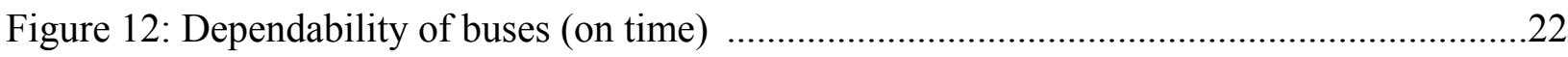

Figure 13: Cleanliness \& Comfort..............................................................................22

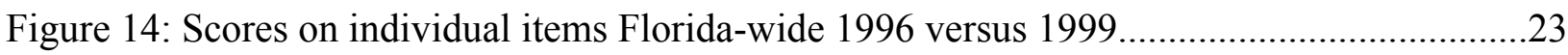

Figure 15: All Systems - Correlation of Income with satisfaction items..................................24

Figure 16: All Systems - Correlation of Age with satisfaction items .......................................26

Figure 17: All Systems - Correlation of Auto ownership with satisfaction items .....................28

Figure 18: Key West Customer Satisfaction Model ...........................................................48

Figure 19: Key West Correlation of income with satisfaction items .......................................49

Figure 20: Key West Correlation of age with satisfaction items ..............................................51

Figure 21: Key West Correlation of auto ownership with satisfaction items .............................53

Figure 22: Key West Importance / Performance Matrix ........................................................55

Figure 23: JTA Customer Satisfaction Model ......................................................................65

Figure 24: JTA Correlation of income with satisfaction items ..............................................66

Figure 25: JTA Correlation of age with satisfaction items ...................................................68

Figure 26: JTA Correlation of auto ownership with satisfaction items .....................................70

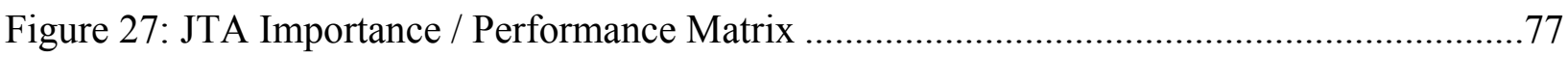

Figure 28: MCAT 1994 Customer Satisfaction Model...............................................................85

Figure 29: MCAT 1994 Importance / Performance Matrix.........................................................86

Figure 30: MCAT 1998 Customer Satisfaction Model.........................................................91

Figure 31: MCAT 1998 Correlation of income with satisfaction items ...................................92

Figure 32: MCAT 1998 Correlation of age with satisfaction items ..........................................94

Figure 33: MCAT 1998 Correlation of auto ownership with satisfaction items .......................96

Figure 34: MCAT 1998 Importance / Performance Matrix ....................................................99

Figure 35: PalmTran Customer Satisfaction Model ..............................................................108

Figure 36: PalmTran Correlation of income with satisfaction items .....................................109 
Figure 37: PalmTran Correlation of age with satisfaction items

Figure 38: PalmTran Correlation of auto ownership with satisfaction items .112

Figure 39: PalmTran Importance / Performance Matrix .120

Figure 40: RTS Customer Satisfaction Model.... 132

Figure 41: RTS Correlation of income with satisfaction items 133

Figure 42: RTS Correlation of age with satisfaction items. 136

Figure 43: RTS Correlation of auto ownership with satisfaction items. 138

Figure 44: RTS Importance / Performance Matrix 144

Figure 45: SCAT Sarasota Customer Satisfaction Model 153

Figure 46: SCAT Sarasota Correlation of income with satisfaction items ..............................154

Figure 47: SCAT Sarasota Correlation of age with satisfaction items ....................................156

Figure 48: SCAT Sarasota Correlation of auto ownership with satisfaction items ...................158

Figure 49: SCAT Sarasota Importance / Performance Matrix.................................................161

Figure 50: SCAT Brevard Customer Satisfaction Model .....................................................169

Figure 51: SCAT Brevard Correlation of income with satisfaction items...............................170

Figure 52: SCAT Brevard Correlation of age with satisfaction items ....................................172

Figure 53: SCAT Brevard Correlation of auto ownership with satisfaction items....................174

Figure 54: SCAT Brevard Importance / Performance Matrix .................................................177

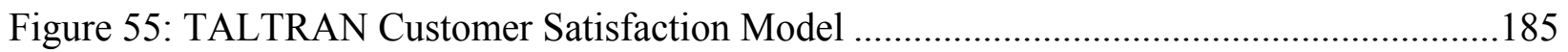

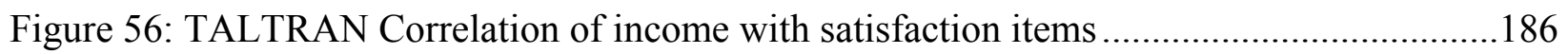

Figure 57: TALTRAN Correlation of age with satisfaction items ........................................188

Figure 58: TALTRAN Correlation of auto ownership with satisfaction items .........................190

Figure 59: TALTRAN Importance / Performance Matrix........................................................193

Figure 60: VOTRAN Customer Satisfaction Model ..........................................................203

Figure 61: VOTRAN Correlation of income with satisfaction items ......................................204

Figure 62: VOTRAN Correlation of age with satisfaction items .......................................206

Figure 63: VOTRAN Correlation of auto ownership with satisfaction items ...........................208

Figure 64: VOTRAN Importance / Performance Matrix...................................................216 



\section{INTRODUCTION}

The purpose of this memorandum is provide an overview of the results and analysis of the data collected in the 1999 Florida Transit Properties Customer Satisfaction Index project, as well as to briefly review the data sources and the weighting methodologies.

\section{OBJECTIVES}

The objectives of the Transit Customer Satisfaction Index project were to provide:

- a systematic evaluation of each participating transit authority's customer satisfaction;

- insight into which factors drive customer satisfaction;

- a comparison of customer satisfaction data from each system with data from other Florida transit systems and other systems in the nation, which will enhance understanding of each system's relative performance; and,

- recommendations for how to increase customer satisfaction.

\section{SELECTED SYSTEMS}

For this study, on-board surveys that had been conducted recently for state of Florida transit properties were used in the development of this index.

The available surveys for this project were:

$\begin{array}{llc}\text { System } & \text { Location } & \text { Date of Survey } \\ \text { MCAT } & \text { Bradenton } & 1994 \\ \text { MCAT } & \text { Bradenton } & 1998 \\ \text { Okaloosa } & \text { Okaloosa County } & 1996 \\ \text { Okaloosa } & \text { Okaloosa County } & 1998 \\ \text { ECAT } & \text { Pensacola } & 1996 \\ \text { LYNX (10 routes) } & \text { Orlando } & 1996 \\ \text { LeeTran } & \text { Fort Myers } & 2000 \\ \text { JTA } & \text { Jacksonville } & 1999 \\ \text { City Transit } & \text { Key West } & 1999 \\ \text { PalmTran } & \text { West Palm Beach } & 1999 \\ \text { SCAT } & \text { Sarasota } & 1999 \\ \text { SCAT (Space Coast) } & \text { Cocoa } & 1999 \\ \text { VOTRAN } & \text { Daytona Beach } & 1999 \\ \text { RTS } & \text { Gainesville } & 1999 \\ \text { TALTRAN } & \text { Tallahassee } & 1999\end{array}$


The survey instruments used in each of the systems are included as Appendix 1.

The approach of using existing on-board surveys removed the need to conduct a massive data collection effort and hence improved the efficiency of this project greatly. As might be expected, there were several data inconsistencies between the surveys that had to be resolved to create indexes. In some cases, the inconsistencies were large enough to cause rejection of survey efforts for inclusion in the index. In other cases, the difficulties were resolved through analysis of the differences between the surveys. This process is detailed below.

\section{$\underline{\text { Ridership Frequencies }}$}

There are several different ways that ridership frequencies were recorded on these surveys. Since one of the initial steps in developing the Transit CSI was to account for different probabilities of sampling people who have different levels of frequency of use (see 1996 Transit Customer Satisfaction index, technical memorandum No. 2), these differences had to be resolved in order to proceed.

The different recording methods arise from the response categories permitted for the question, "On average, how many days a week do you ride the bus?" The different response formats in the various surveys are:

Once per month to 7 days per week

Once per month to 6 days per week

Less than 1 day per week to 6 days per weekRTS

$1,2-3,4+$ days per week or once every
TALTRAN, City Transit, VOTRAN, JTA

SCAT Sarasota, SCAT Brevard,

\section{_ weeks}

MCAT, PalmTran, LYNX,

Okaloosa 
The greatest difficulty rests with the systems where only '4+' days was recorded. Analysis of the data from systems where people were asked if they rode the bus 0 to 7 days per week provides the following data:

Percent Ride

$\underline{\text { System }} \underline{0 \text { days }} \underline{1 \text { day }} \underline{2 \text { days }} \underline{3 \text { days }} \underline{4 \text { days }} \underline{5 \text { days }} \underline{6 \text { days }} \underline{7 \text { days }}$

$\begin{array}{lllllllll}\text { JTA } & 2.5 & 3.4 & 6.1 & 7.8 & 9.2 & 40.4 & 16.9 & 13.8\end{array}$

$\begin{array}{llllllll}\text { Key West } & 4.1 & \text { Comb. } 11.8 & 13.3 & 10.7 & 24.0 & 10.7 & 19.4\end{array}$

$\begin{array}{llllllll}\text { TALTRAN } & 5.3 & \text { Comb. } 10.1 & 7.7 & 9.6 & 40.6 & 12.6 & 14.1\end{array}$

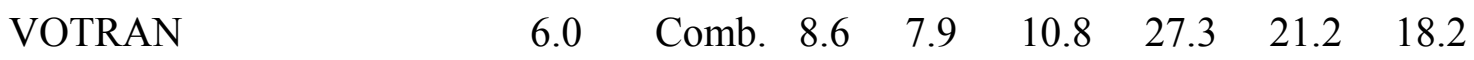

From this data, the best approximation for all riders in systems where anything over 4 days was not specified is approximately 5.5 days for all. Although this is not as precise as one might like, this will serve to provide appropriate probability sampling weights for ridership in those systems. The actual value of the weights only differs slightly in those ranges so the effects should be minimal. Certainly it is not the objective of this project to dismiss data where the frequency of ridership does not match the ideal characteristics, where so much other valuable data is available with these minor adjustments.

\section{$\underline{\text { Satisfaction Items }}$}

The differences in satisfaction items on the surveys provided a potentially much more serious impact on the process of developing the index. The surveys do not contain an identical set of questions, so it is vital to distinguish which questions appear in which surveys and the extent to which non-identical surveys can be used to create the index. 
The table below summarizes the questions asked on the surveys.

Table 1

1999 Transit Customer Satisfaction Index On-Board Questionnaire Item Matrix

\begin{tabular}{|c|c|c|c|c|c|c|c|c|c|c|c|c|}
\hline Question & SCAT & TALTRAN & JTA & $\begin{array}{l}\text { Palm } \\
\text { Tran }\end{array}$ & $\begin{array}{l}\text { Key } \\
\text { West }\end{array}$ & VOTRAN & RTS & $\begin{array}{l}\text { MCAT } \\
94 \\
\end{array}$ & $\begin{array}{l}\text { MCAT } \\
98 \\
\end{array}$ & LYNX & Okaloosa & SCATBrev \\
\hline $\begin{array}{l}\text { satisfaction } \\
\text { beginning }\end{array}$ & & & $\mathrm{x}$ & & $\mathrm{x}$ & $\mathrm{x}$ & $\mathrm{X}$ & & & & & \\
\hline $\begin{array}{l}\text { satisfaction @ } \\
\text { end }\end{array}$ & $\mathrm{x}$ & $\mathrm{x}$ & & $\mathrm{x}$ & & & & $\mathrm{X}$ & $\mathrm{x}$ & $\mathrm{X}$ & $\mathrm{x}$ & $\mathrm{x}$ \\
\hline days of service & $\mathrm{x}$ & $\mathrm{x}$ & & & & & & $\mathrm{x}$ & $\mathrm{x}$ & & & $\mathrm{X}$ \\
\hline hours of service & $\mathrm{x}$ & $\mathrm{x}$ & & & & & & $\mathrm{x}$ & $\mathrm{x}$ & $\mathrm{x}$ & $\begin{array}{c}\mathrm{x} \\
\text { (runs when } \\
\text { I need it) }\end{array}$ & $\mathrm{x}$ \\
\hline $\begin{array}{l}\text { time of day the } \\
\text { earliest buses run } \\
\text { on weekdays }\end{array}$ & & & $\mathrm{x}$ & $\mathrm{x}$ & $\mathrm{x}$ & $\mathrm{x}$ & $\mathrm{x}$ & & & & & \\
\hline $\begin{array}{l}\text { time of day the } \\
\text { latest buses run } \\
\text { on weekdays }\end{array}$ & & & $\mathrm{x}$ & $\mathrm{x}$ & $\mathrm{x}$ & $\mathrm{x}$ & $\mathrm{x}$ & & & & & \\
\hline $\begin{array}{l}\text { time of day the } \\
\text { earliest buses run } \\
\text { on weekends }\end{array}$ & & & $\mathrm{x}$ & $\mathrm{x}$ & $\mathrm{x}$ & $\mathrm{x}$ & $\mathrm{x}$ & & & & & \\
\hline $\begin{array}{l}\text { time of day the } \\
\text { latest buses run } \\
\text { on weekends }\end{array}$ & & & $\mathrm{x}$ & $\mathrm{x}$ & $\mathrm{x}$ & $\mathrm{x}$ & $\mathrm{x}$ & & & & & \\
\hline $\begin{array}{ll}\text { frequency } \\
\text { service }\end{array}$ & $\mathrm{x}$ & $\mathrm{x}$ & $\mathrm{x}$ & $\mathrm{x}$ & $\mathrm{x}$ & $\mathrm{x}$ & $\mathrm{x}$ & $\mathrm{x}$ & $\mathrm{x}$ & $\mathrm{x}$ & $\begin{array}{l}\mathrm{x} \text { (wait } \\
\text { time) }\end{array}$ & $\mathrm{x}$ \\
\hline $\begin{array}{l}\text { convenience of } \\
\text { routes }\end{array}$ & $\mathrm{x}$ & $\mathrm{x}$ & & & & & & $\mathrm{X}$ & $\mathrm{x}$ & $\mathrm{X}$ & $\begin{array}{l}\mathrm{x} \text { (arrange } \\
\text { trips) }\end{array}$ & $\mathrm{x}$ \\
\hline $\begin{array}{l}\text { your ability to get } \\
\text { where you want } \\
\text { to go }\end{array}$ & & & $\mathrm{x}$ & $\mathrm{x}$ & $\mathrm{x}$ & $\mathrm{X}$ & $\mathrm{x}$ & & & & & \\
\hline
\end{tabular}




\begin{tabular}{|c|c|c|c|c|c|c|c|c|c|c|c|c|}
\hline \multicolumn{13}{|c|}{ 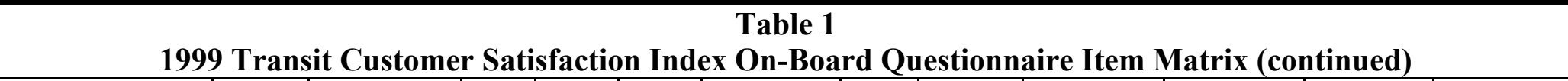 } \\
\hline Question & SCAT & TALTRAN & JTA & $\begin{array}{l}\text { Palm } \\
\text { Tran }\end{array}$ & $\begin{array}{l}\text { Key } \\
\text { West }\end{array}$ & VOTRAN & RTS & $\begin{array}{l}\text { MCAT } \\
94\end{array}$ & MCAT 98 & LYNX & Okaloosa & SCATBrev \\
\hline $\begin{array}{l}\text { dependability of } \\
\text { buses (on time) }\end{array}$ & $\mathrm{x}$ & $\mathrm{x}$ & & & & & & $\mathrm{x}$ & $\mathrm{x}$ & & $\mathrm{x}$ & $\mathrm{x}$ \\
\hline $\begin{array}{l}\text { How regularly } \\
\text { buses arrive on } \\
\text { time }\end{array}$ & & & $\mathrm{x}$ & $\mathrm{x}$ & $\mathrm{x}$ & $\mathrm{x}$ & $\mathrm{x}$ & & & & & \\
\hline $\begin{array}{ll}\text { travel time on } \\
\text { buses }\end{array}$ & $\mathrm{x}$ & $\mathrm{x}$ & $\mathrm{x}$ & $\mathrm{x}$ & $\mathrm{x}$ & $\mathrm{x}$ & $\mathrm{x}$ & $\mathrm{x}$ & $\mathrm{x}$ & $\mathrm{x}$ & & $\mathrm{x}$ \\
\hline $\begin{array}{l}\text { cost of riding the } \\
\text { bus }\end{array}$ & $\mathrm{x}$ & $\mathrm{x}$ & $\mathrm{x}$ & $\mathrm{x}$ & $\mathrm{x}$ & $\mathrm{x}$ & $\mathrm{x}$ & $\mathrm{x}$ & $\mathrm{x}$ & & $\mathrm{x}$ & $\mathrm{x}$ \\
\hline $\begin{array}{l}\text { availability of bus } \\
\text { route } \\
\text { information/maps }\end{array}$ & $\mathrm{x}$ & $\mathrm{x}$ & & $\mathrm{x}$ & $\mathrm{x}$ & $\mathrm{x}$ & $\mathrm{x}$ & $\mathrm{x}$ & $\mathrm{x}$ & & & $\mathrm{x}$ \\
\hline $\begin{array}{l}\text { usefulness of bus } \\
\text { route info/maps }\end{array}$ & $\mathrm{x}$ & $\mathrm{x}$ & $\mathrm{x}$ & $\mathrm{x}$ & $\mathrm{x}$ & $\mathrm{x}$ & $\mathrm{x}$ & & $\mathrm{x}$ & & & $\mathrm{x}$ \\
\hline $\begin{array}{l}\text { Vehicle } \\
\text { cleanliness } \quad \& \\
\text { comfort }\end{array}$ & $\mathrm{x}$ & $\mathrm{x}$ & & & & & & $\mathrm{x}$ & $\mathrm{x}$ & & $\mathrm{X}$ & $\mathrm{x}$ \\
\hline $\begin{array}{l}\text { temperature } \\
\text { inside the bus }\end{array}$ & & & $\mathrm{x}$ & $\mathrm{x}$ & $\mathrm{x}$ & $\mathrm{x}$ & $\mathrm{x}$ & & & & & \\
\hline $\begin{array}{l}\text { how clean bus } \\
\text { stops \& buses are }\end{array}$ & & & $\mathrm{x}$ & $\mathrm{x}$ & & $\mathrm{x}$ & $\mathrm{x}$ & & & & & \\
\hline $\begin{array}{l}\text { availability of } \\
\text { seats on the buses }\end{array}$ & & & $\mathrm{x}$ & $\mathrm{x}$ & $\mathrm{x}$ & $\mathrm{x}$ & $\mathrm{x}$ & & & & & \\
\hline operator courtesy & $\mathrm{x}$ & $\mathrm{x}$ & $\mathrm{x}$ & $\mathrm{x}$ & $\mathrm{x}$ & $\mathrm{x}$ & $\mathrm{x}$ & $\mathrm{x}$ & $\mathrm{x}$ & & $\mathrm{x}$ & $\mathrm{x}$ \\
\hline $\begin{array}{l}\text { safety on bus \& } \\
\text { at bus stops }\end{array}$ & $\mathrm{x}$ & $\mathrm{x}$ & $\mathrm{x}$ & $\mathrm{x}$ & $\mathrm{x}$ & $\mathrm{x}$ & $\mathrm{x}$ & $\mathrm{x}$ & $\mathrm{x}$ & & & $\mathrm{x}$ \\
\hline $\begin{array}{l}\text { safety after } \\
\text { getting off bus }\end{array}$ & & & $\mathrm{x}$ & $\mathrm{x}$ & $\mathrm{x}$ & $\mathrm{x}$ & $\mathrm{x}$ & & & & & \\
\hline $\begin{array}{l}\text { transferring } \quad \mathrm{b} / \mathrm{t} \\
\text { buses }\end{array}$ & $\mathrm{x}$ & $\mathrm{x}$ & $\mathrm{x}$ & $\mathrm{x}$ & $\mathrm{x}$ & $\mathrm{x}$ & $\mathrm{x}$ & & $\mathrm{x}$ & $\mathrm{x}$ & & $\mathrm{x}$ \\
\hline $\begin{array}{l}\text { bus operator's } \\
\text { ability to drive } \\
\text { the bus }\end{array}$ & & & $\mathrm{x}$ & $\mathrm{x}$ & $\mathrm{x}$ & $\mathrm{x}$ & $\mathrm{x}$ & & & & & \\
\hline
\end{tabular}


Many of the differences between the surveys amount to the level of detail for the topic in question. For instance, where some surveys contain questions where the customer responds by asking "days of service" and "hours of service," others require customers to rate their satisfaction with the time of day buses leave earliest and latest on weekdays and weekends, creating four separate ratings instead of two. Another example of this is the question rating 'Vehicle cleanliness and comfort' compared to separate questions on temperature inside the bus, how clean buses and bus stops are, and availability of seats on buses.

Other sources of differences are minor wording changes. So where on set of surveys have questions about "convenience of routes" others have questions rating the customers' satisfaction with "your ability to get where you want to go." Another example is "dependability of buses (on time)" versus "How regularly buses arrive on time."

Finally, some surveys have additional questions on similar topics. One set of surveys has a question about "safety on bus and at bus stops." Another set has both that question and one about "safety after getting off bus."

The factor analysis of surveys with similar question sets should reduce these down to the same dimensions. This hypothesis will be examined through separate factor analyses of the two predominant questionnaire format results. If the same basic factors are arrived at, the factor scores should then be comparable. The only potential problem will be if one of the elements in a detailed set of questions (such as "time latest buses run on weekends") turns out to have a markedly different rating than others, thus resulting in a different factor score. This too will be carefully examined in a comparison of the results.

The factor analysis, conducting parallel analyses between the systems using one type of survey format and the set of systems using the second type, indicates that the safety issues are treated much the same way in the two surveys, as they load on to factors with comfort and driver. The span of service issues, however, are not so simple. The span of service when presented as earliest/latest weekday/weekend, is it's own factor. When span of service is hours of service and days of service, it loads together with frequency of service, as a kind of 'system scheduling' factor. In the first instance, when we have earliest/latest weekday/weekend, the frequency loads on with items on convenience of routes and time to make trip. The differences in how these factors are constructed 
indicate that the two are not directly comparable, and that any comparison between the different forms of measuring span of service using the index could be very misleading. Hence comparison on those items will be limited to comparisons with other systems that used the same question format.

Another aspect of the surveys that will impact the development of the index is the varying response formats for the satisfaction items. Surveys for SCAT, SCATBrev, MCAT 1994 and 1998 have a 5 category response format, "very good," "good," "fair," "poor," and "very poor." While surveys for JTA, City Transit, VOTRAN have a 5 category response format rated from "very satisfied" to "neutral" to "very unsatisfied." However, these two different 5 category response format can be validly compared because the responses are likely to be similarly interpreted by customers.

In contrast, the PalmTran survey is a 6 category response format which include, "very good," "good," "neutral," "poor," "very poor," and "don't know." Although the first 5 response categories seem comparable to the surveys described above, the last response, "don't know" must be carefully examined prior to conducting tests. The proportion of 'don't know responses is very low, however, and generally distribution patterns across the response categories are similar to the surveys where this question format is not used.

Lynx is the only survey with a 4 category response format, "very good," "good," "fair," and "poor." In addition, the Lynx data is a sample of only 10 routes in the entire system and therefore, it will not be used to calculate the index. Other surveys eliminated from the index due to sampling problems are LeeTran, Bay Town, and Okaloosa. Bay Town is a trolley services that are highly utilized by tourist so their responses are not likely to be representative of local opinions about service satisfaction. Okaloosa is a van service that customers contact when rides are needed. This type of service is distinct from the other systems so their customers' interpretation of the satisfaction items are likely to differ from those in the other systems. The LeeTran data turns out to be the same data that was used in the prior CSI effort. 
In summary, the survey efforts used in this analysis were the following:

\begin{tabular}{llcr} 
System & Location & Date of Survey & Total Surveys \\
\cline { 2 - 3 } MCAT & Bradenton & 1994 & 736 \\
MCAT & Bradenton & 1998 & 655 \\
JTA & Jacksonville & 1999 & 4,733 \\
City Transit & Key West & 1999 & 200 \\
PalmTran & West Palm Beach & 1999 & 3,090 \\
SCAT & Sarasota & 1999 & 1,250 \\
SCAT (Space Coast) & Cocoa & 1999 & 422 \\
VOTRAN & Daytona Beach & 1999 & 1,972 \\
RTS & Gainesville & 1999 & 2,107 \\
TALTRAN & Tallahassee & 1999 & 1,446
\end{tabular}

\section{Handling of the Ridership Frequency Response Bias}

In Technical Memorandum Number two for the 1996 Transit CSI project, a particular difficulty in sampling is described, where higher frequency riders are more likely to be surveyed in an on-board surveying effort than low frequency riders. CUTR's analysis of the on-board representation problem yields a simple method for creating a rough estimate of the proper weighting for each response. The problem can be illustrated with the following example.

Suppose bus ridership for a particular route has frequency of use characteristics as described in Table 3 below. If we assume equal trips per day for each category of use, the percentage of all system trips by each category of use can be calculated with the following formula:

(Equation 1): $\quad \%$ of trips by users in category $I=$

\section{(\% of riders in category $I) /($ frequency of use by category $I$ )}

$\Sigma(\%$ of riders in category $I) /($ frequency of use by category $I)$ for all $I$

For those who use the system once per week, the formula would yield the result from Equation 1:

$((35$ percent $) @ 1$ day $/$ week $)) /(.35 * 5+.1 * 4+.1 * 3+.1 * 2+.35 * 1)=(.35 / 3)=11.7 \%$ 
Application of the formula to each category yields the results in the right hand column of Table 2 below.

\begin{tabular}{|c|c|c|}
\hline \multicolumn{3}{|c|}{ Table 2 } \\
Relationship of Rider Use Frequency to Percentage of Trips Taken \\
\hline Frequency of Use & Percentage of Riders & Percentage of Trips \\
\hline 5/week & $35 \%$ & $58 \%$ \\
\hline 4/week & $10 \%$ & $13 \%$ \\
\hline 3/week & $10 \%$ & $10 \%$ \\
\hline 2/week & $10 \%$ & $7 \%$ \\
\hline 1/week & $35 \%$ & $12 \%$ \\
\hline
\end{tabular}

Any sampling plan that distributes surveys randomly to riders on a bus (or people waiting for a bus) will necessarily result in survey returns that are proportional to the trips taken by each category of rider, rather than to the percentage of the overall system ridership. In this admittedly extreme example, it is clear that the ridership would not be properly represented.

To minimize this problem, CUTR utilized a weighting scheme based on the respondents' self-assessment of frequency of bus ridership. Respondents were asked to note on which of the last seven days (Monday through Sunday) they had ridden the bus. Using the answers to these questions, CUTR determined the probability that each frequency category would have been surveyed and, from that probability and the total number of responses for each category, estimated the distribution of riders in each frequency category. Weights were assigned by dividing the estimated number of riders by the actual percentage of responses for each frequency category.

The exact formula for estimating the total distribution of weekday riders is then determined with the following formula:

$\%$ of riders in category $I=$

$\%$ of surveys returned by category $I /$ Frequency of use by category $I$

$\sum((\%$ of surveys returned by category $I) /($ Frequency of use by category $I))$ 
for all $I$

These results were analyzed for the system as a whole only, since route-level results were not required for this project.

\section{System weights}

To compare results across systems, an additional weighting scheme had to be developed to account for differences in number of responses across systems. Three weighting schemes were available:

1. No weighting

2. Weight by system ridership

3. Weight by area population

Among these, it seemed most logical to weight by system ridership, thus allowing larger systems (such as JTA and PalmTran) to affect overall scores more than smaller systems, which in turn is more representative of attitudes among all Florida riders. Ridership data were drawn from CUTR's 1999 peer evaluation of all Florida systems. 


\section{RESULTS INDEXING}

Following the weighting of the survey results, mean scores were calculated for each of the satisfaction items for each system. To create the overall mean between systems, the system weight (described in the previous section) was also used. Each of the individual system means for each item was divided by the overall mean and then multiplied by 100 .

For example, on the row summarizing Overall Satisfaction results, the weighted mean for all six systems was a 3.88 average. For BCT, the mean was 3.59, which resulted in an index score of $3.59 / 3.88$, or 0.9261 , which is then converted to 92.61 for the index score. Index scores for the other systems and on the other items were calculated in a similar fashion.

The results are summarized in the Table 3 on the following page. 


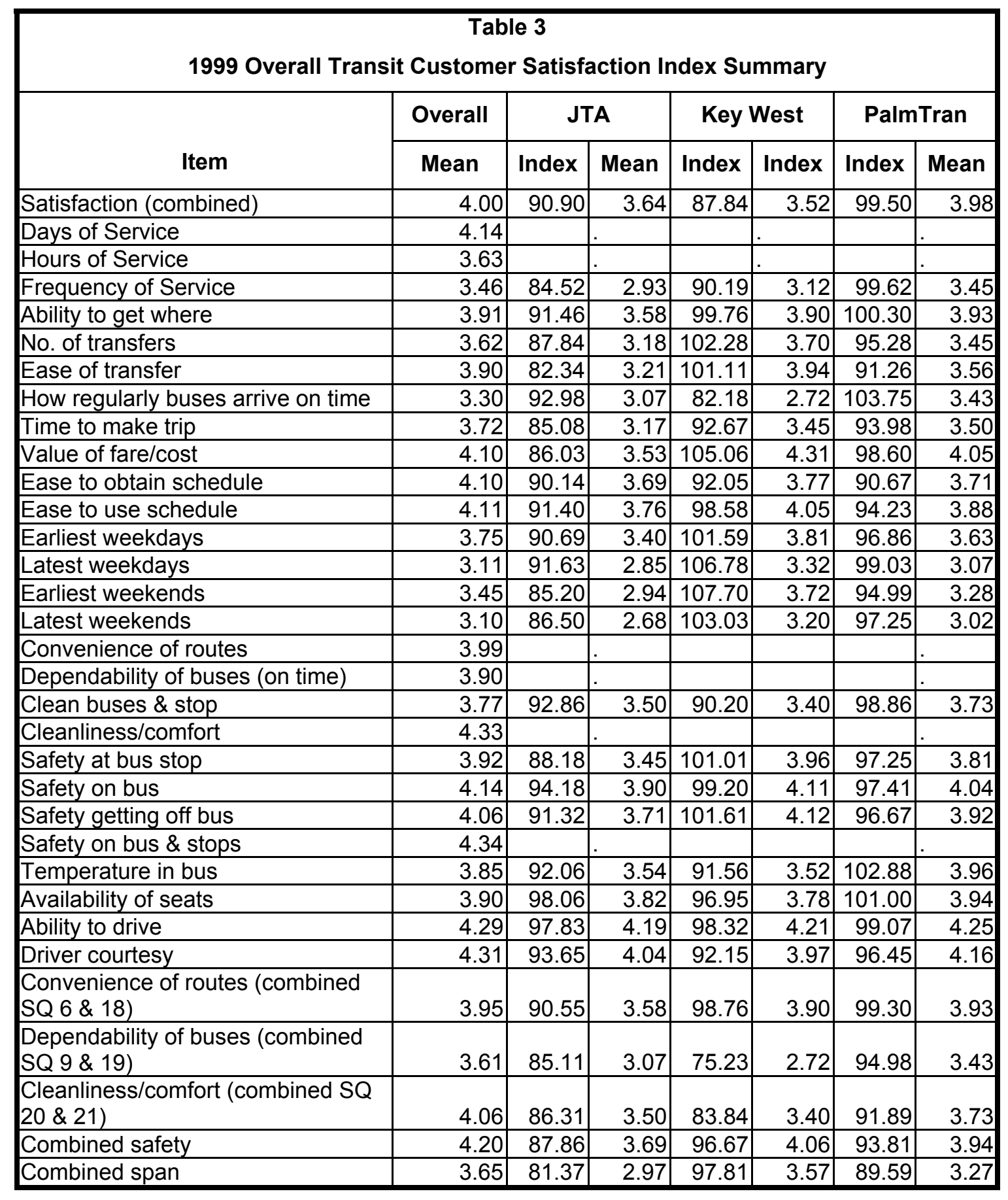




\begin{tabular}{|c|c|c|c|c|c|}
\hline \multicolumn{6}{|c|}{$\begin{array}{c}\text { Table } 3 \\
1999 \text { Overall Transit Customer Satisfaction Index Summary (continued) }\end{array}$} \\
\hline \multirow[b]{2}{*}{ Item } & \multirow{2}{*}{$\begin{array}{c}\text { Overall } \\
\text { Mean }\end{array}$} & \multicolumn{2}{|c|}{ MCAT 1994} & \multicolumn{2}{|c|}{ MCAT 1998} \\
\hline & & Index & Mean & Index & Mean \\
\hline Satisfaction (combined) & 4.00 & 105.16 & 4.21 & 102.85 & 4.12 \\
\hline Days of Service & 4.14 & 102.42 & 4.24 & 96.75 & 4.01 \\
\hline Hours of Service & 3.63 & 105.01 & 3.81 & 99.61 & 3.61 \\
\hline Frequency of Service & 3.46 & 111.17 & 3.85 & 108.05 & 3.74 \\
\hline Ability to get where & 3.91 & & & & \\
\hline No. of transfers & 3.62 & & & & \\
\hline Ease of transfer & 3.90 & & & 108.71 & 4.24 \\
\hline How regularly buses arrive on time & 3.30 & & & & \\
\hline Time to make trip & 3.72 & 105.33 & 3.92 & 104.70 & 3.90 \\
\hline Value of fare/cost & 4.10 & 96.07 & 3.94 & 101.59 & 4.17 \\
\hline Ease to obtain schedule & 4.10 & 105.75 & 4.33 & 105.53 & 4.32 \\
\hline Ease to use schedule & 4.11 & & & 106.56 & 4.38 \\
\hline Earliest weekdays & 3.75 & & & & \\
\hline Latest weekdays & 3.11 & & & & \\
\hline Earliest weekends & 3.45 & & & & \\
\hline Latest weekends & 3.10 & & & & \\
\hline Convenience of routes & 3.99 & 101.73 & 4.06 & 102.10 & 4.08 \\
\hline Dependability of buses (on time) & 3.90 & 101.27 & 3.95 & 97.67 & 3.81 \\
\hline Clean buses \& stop & 3.77 & & & & \\
\hline Cleanliness/comfort & 4.33 & 99.52 & 4.31 & 101.98 & 4.41 \\
\hline Safety at bus stop & 3.92 & & & & \\
\hline Safety on bus & 4.14 & & & & \\
\hline Safety getting off bus & 4.06 & & & & \\
\hline Safety on bus \& stops & 4.34 & 99.50 & 4.31 & 102.11 & 4.43 \\
\hline Temperature in bus & 3.85 & & & & \\
\hline Availability of seats & 3.90 & & & & \\
\hline Ability to drive & 4.29 & & & & \\
\hline Driver courtesy & 4.31 & 104.16 & 4.49 & 102.78 & 4.43 \\
\hline $\begin{array}{l}\text { Convenience of routes (combined } \\
\text { SQ } 6 \text { \& 18) }\end{array}$ & 3.95 & 102.73 & 4.06 & 103.10 & 4.08 \\
\hline $\begin{array}{l}\text { Dependability of buses (combined } \\
\text { SQ } 9 \text { \& 19) }\end{array}$ & 3.61 & 109.49 & 3.95 & 105.60 & 3.81 \\
\hline $\begin{array}{l}\text { Cleanliness/comfort (combined SQ } \\
20 \text { \& 21) }\end{array}$ & 4.06 & 106.14 & 4.31 & 108.76 & 4.41 \\
\hline Combined safety & 4.20 & 104.05 & 4.37 & 105.48 & 4.43 \\
\hline Combined span & 3.65 & 110.41 & 4.03 & 104.11 & 3.80 \\
\hline
\end{tabular}




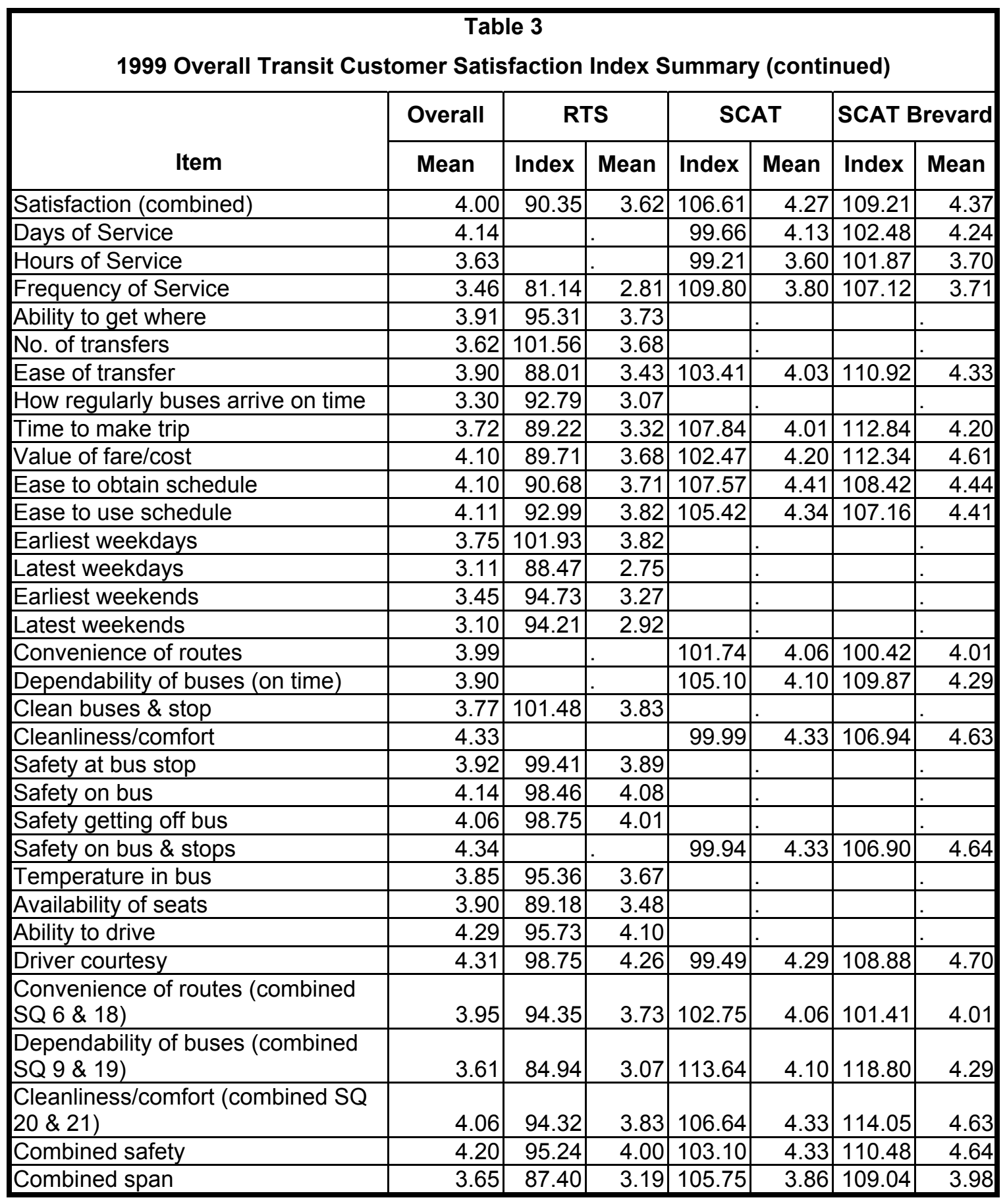




\begin{tabular}{|c|c|c|c|c|c|}
\hline \multicolumn{6}{|c|}{$\begin{array}{c}\text { Table } 3 \\
1999 \text { Overall Transit Customer Satisfaction Index Summary (continued) }\end{array}$} \\
\hline \multirow[b]{2}{*}{ Item } & \multirow{2}{*}{$\frac{\text { Overall }}{\text { Mean }}$} & \multicolumn{2}{|c|}{ TALTRAN } & \multicolumn{2}{|c|}{ VOTRAN } \\
\hline & & Index & Mean & Index & Mean \\
\hline Satisfaction (combined) & 4.00 & 97.18 & 3.89 & 110.45 & 4.42 \\
\hline Days of Service & 4.14 & 98.74 & 4.09 & & \\
\hline Hours of Service & 3.63 & 94.37 & 3.42 & & \\
\hline Frequency of Service & 3.46 & 98.23 & 3.40 & 109.54 & 3.79 \\
\hline Ability to get where & 3.91 & & & 111.18 & 4.35 \\
\hline No. of transfers & 3.62 & & & 108.45 & 3.93 \\
\hline Ease of transfer & 3.90 & 98.69 & 3.85 & 110.74 & 4.32 \\
\hline How regularly buses arrive on time & 3.30 & & & 126.40 & 4.18 \\
\hline Time to make trip & 3.72 & 99.60 & 3.71 & 106.36 & 3.96 \\
\hline Value of fare/cost & 4.10 & 100.55 & 4.13 & 106.53 & 4.37 \\
\hline Ease to obtain schedule & 4.10 & 97.02 & 3.97 & 108.43 & 4.44 \\
\hline Ease to use schedule & 4.11 & 98.74 & 4.06 & 103.18 & 4.24 \\
\hline Earliest weekdays & 3.75 & & & 105.85 & 3.97 \\
\hline Latest weekdays & 3.11 & & & 112.45 & 3.49 \\
\hline Earliest weekends & 3.45 & & & 112.59 & 3.89 \\
\hline Latest weekends & 3.10 & & & 114.72 & 3.56 \\
\hline Convenience of routes & 3.99 & 94.25 & 3.76 & & \\
\hline Dependability of buses (on time) & 3.90 & 86.96 & 3.39 & & \\
\hline Clean buses \& stop & 3.77 & & & 113.25 & 4.27 \\
\hline Cleanliness/comfort & 4.33 & 92.44 & 4.00 & & \\
\hline Safety at bus stop & 3.92 & & & 110.53 & 4.33 \\
\hline Safety on bus & 4.14 & & & 108.24 & 4.48 \\
\hline Safety getting off bus & 4.06 & & & 108.57 & 4.41 \\
\hline Safety on bus \& stops & 4.34 & 92.47 & 4.01 & & \\
\hline Temperature in bus & 3.85 & & & 116.04 & 4.46 \\
\hline Availability of seats & 3.90 & & & 113.88 & 4.44 \\
\hline Ability to drive & 4.29 & & & 107.72 & 4.62 \\
\hline Driver courtesy & 4.31 & 95.58 & 4.12 & 105.21 & 4.54 \\
\hline $\begin{array}{l}\text { Convenience of routes (combined } \\
\text { SQ } 6 \text { \& 18) }\end{array}$ & 3.95 & 95.18 & 3.76 & 110.07 & 4.35 \\
\hline $\begin{array}{l}\text { Dependability of buses (combined } \\
\text { SQ } 9 \text { \& 19) }\end{array}$ & 3.61 & 94.02 & 3.39 & 115.71 & 4.18 \\
\hline $\begin{array}{l}\text { Cleanliness/comfort (combined SQ } \\
20 \text { \& 21) }\end{array}$ & 4.06 & 98.58 & 4.00 & 105.26 & 4.27 \\
\hline Combined safety & 4.20 & 95.48 & 4.01 & 105.00 & 4.41 \\
\hline Combined span & 3.65 & 103.01 & 3.76 & 102.47 & 3.74 \\
\hline
\end{tabular}


Within the Florida-based study, VOTRAN consistently had the highest index ratings on each item. The item with the lowest level of satisfaction in each system, just as in the first Transit CSI project, was the time of the latest weekend runs (mean of 3.10 across all systems), whereas the highest rated item of those asked in all systems was driver courtesy (mean of 4.31 across all systems).

Overall satisfaction scores for the systems are presented below:

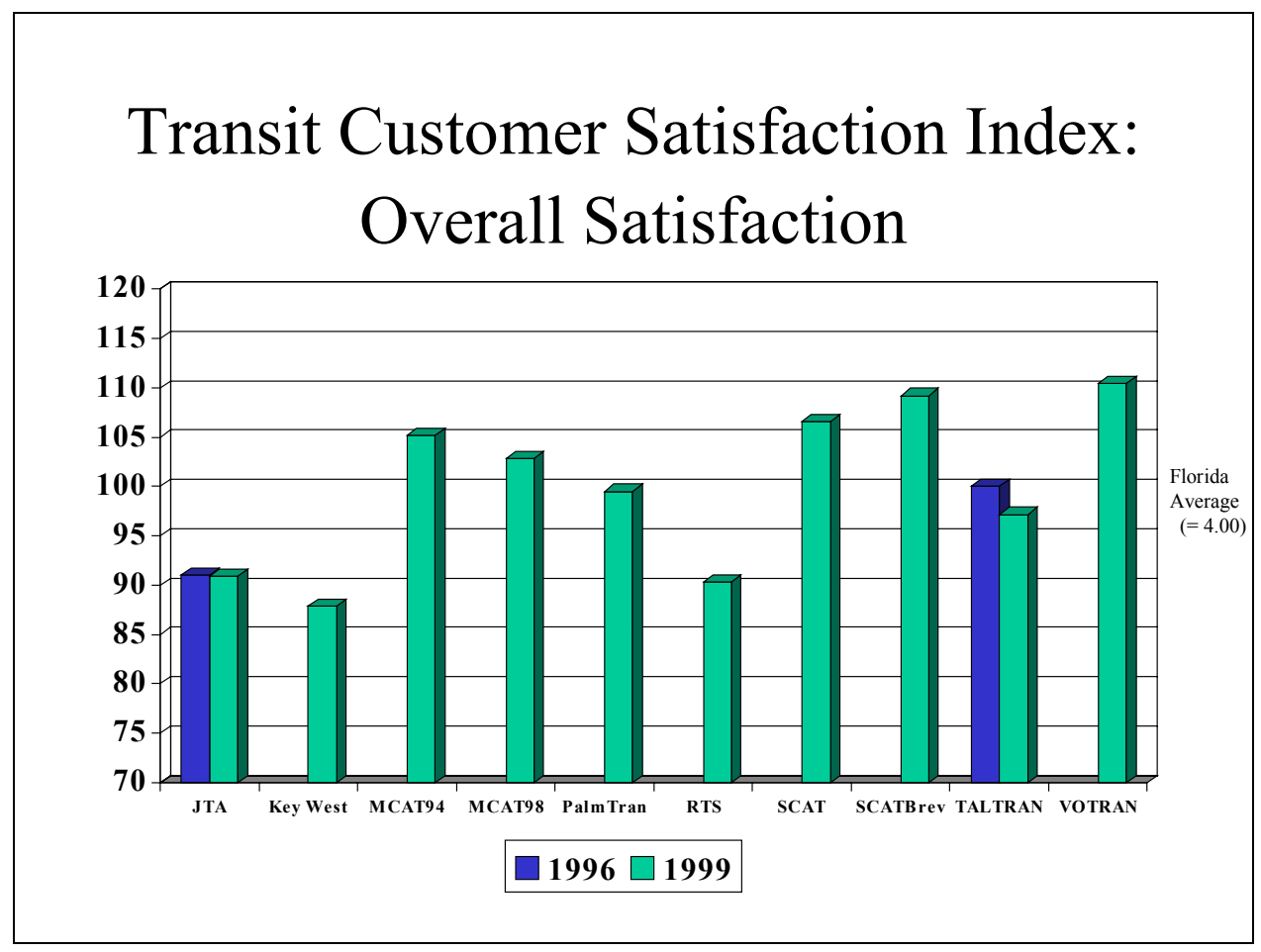

Figure 1: Overall Satisfaction

The index ratings for those items where data was obtained from all systems are provided in chart form on the following pages. 


\section{Transit Customer Satisfaction Index: Frequency of service}

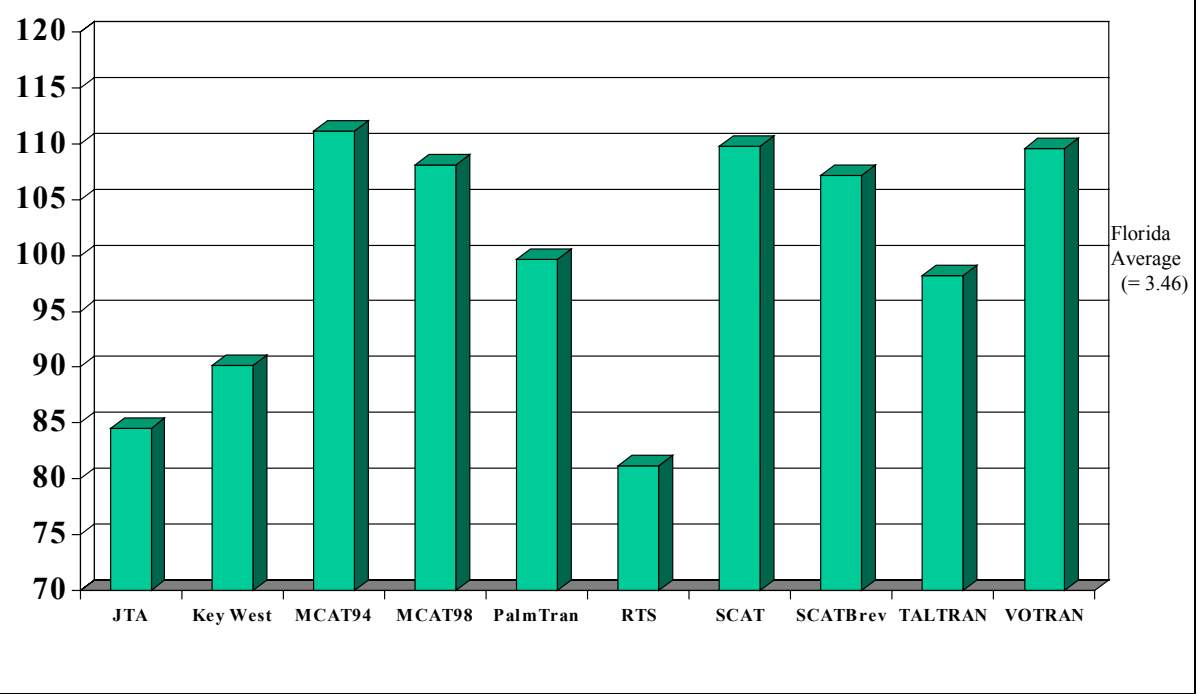

Figure 2: Frequency of service

\section{Transit Customer Satisfaction Index: Span of service}

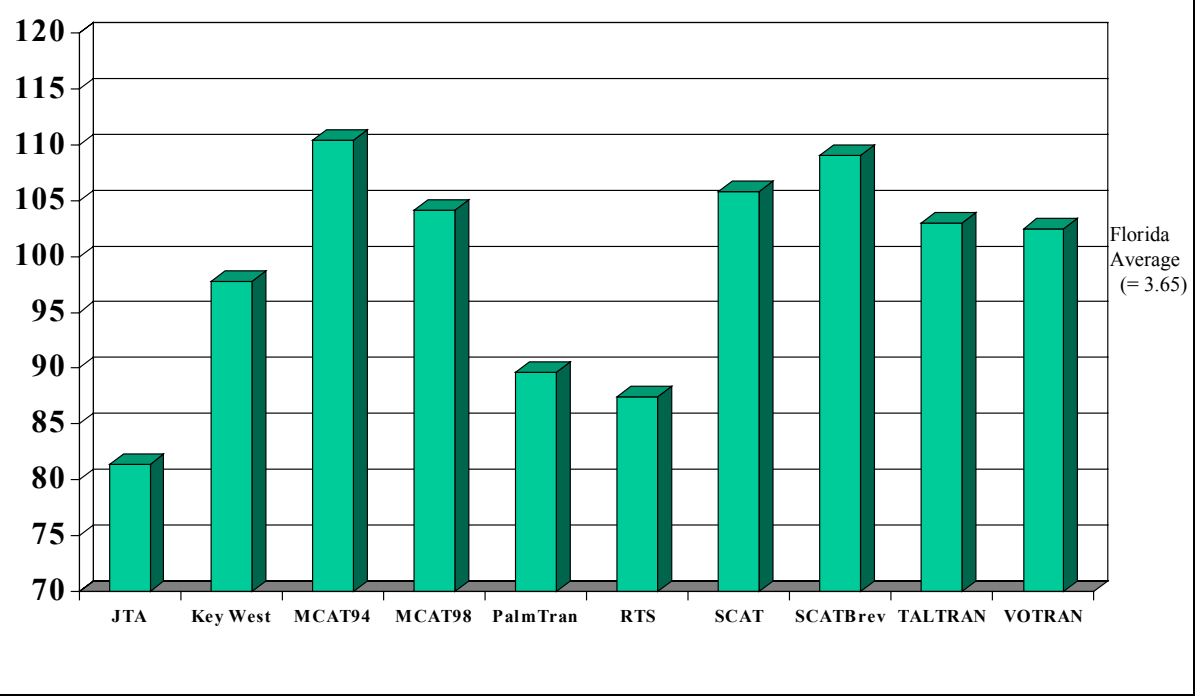

Figure 3: Span of service 


\section{Transit Customer Satisfaction Index: Ease of transferring buses}

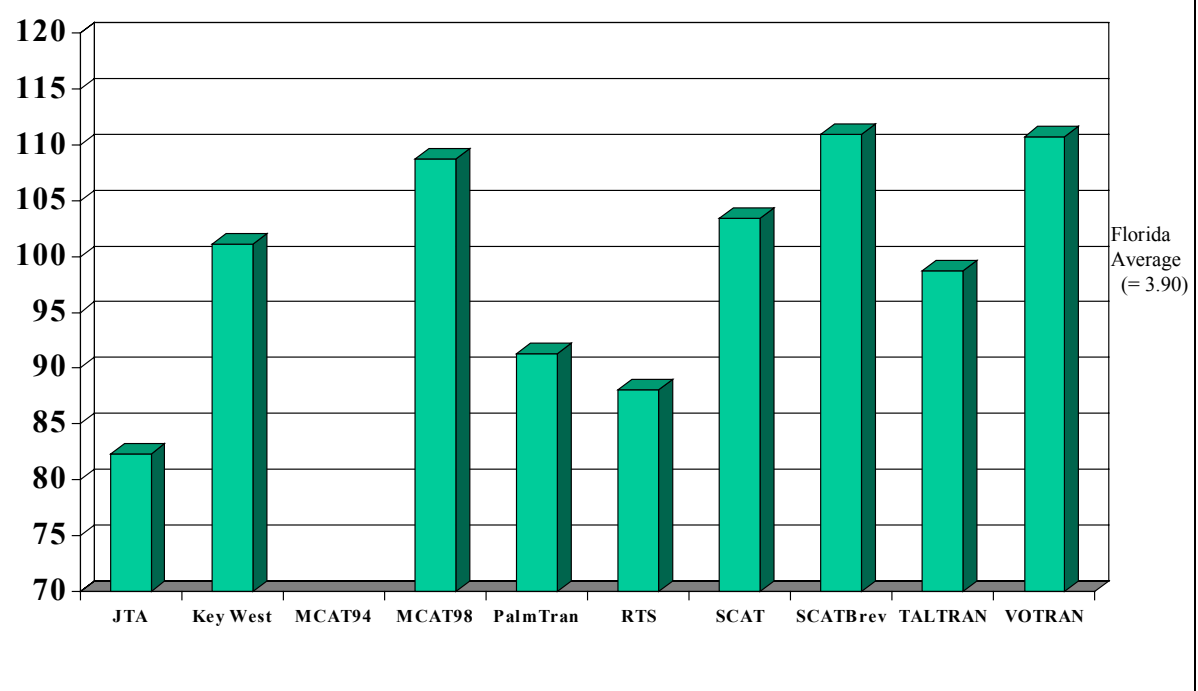

Figure 4: Ease of transferring buses

\section{Transit Customer Satisfaction Index:}

Time to make trips

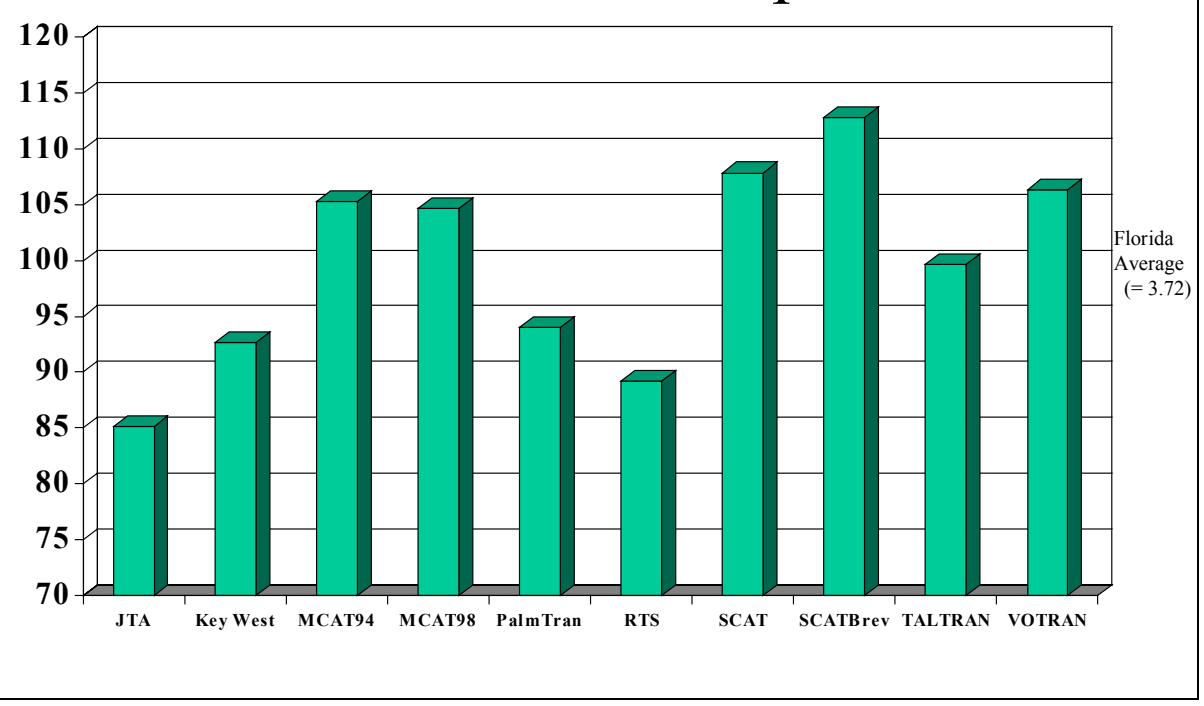

Figure 5: Time to make trips 


\section{Transit Customer Satisfaction Index: Value of fare}

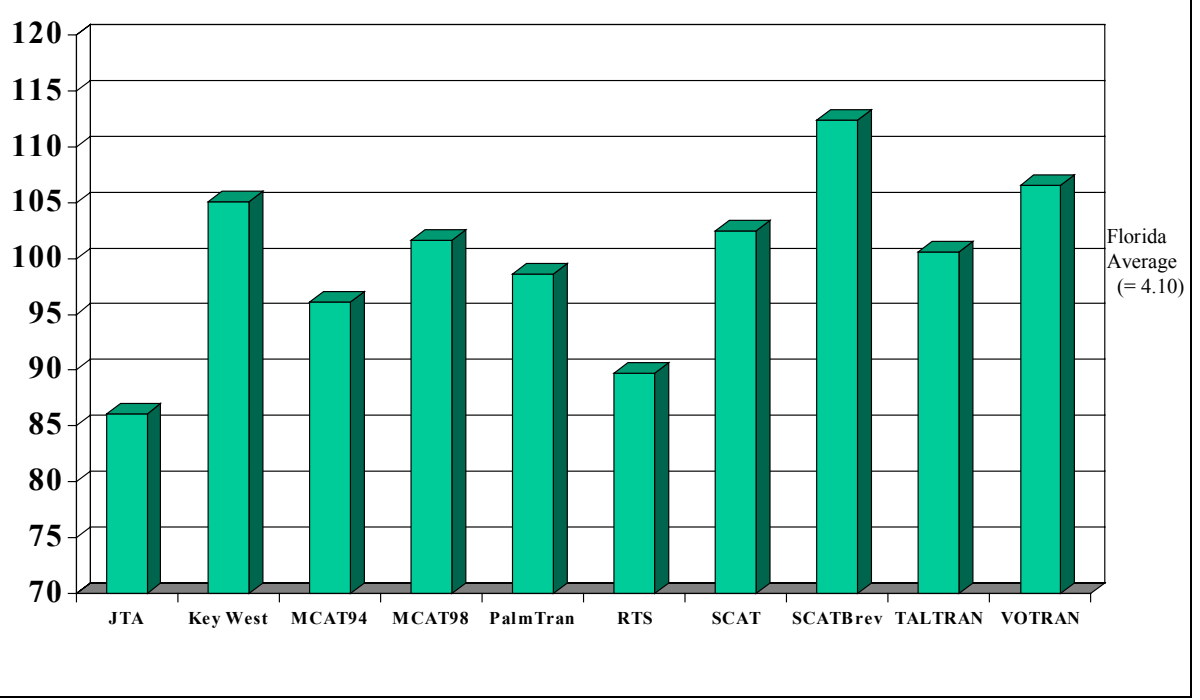

Figure 6: Value of fare

\section{Transit Customer Satisfaction Index: Ease of obtaining schedule}

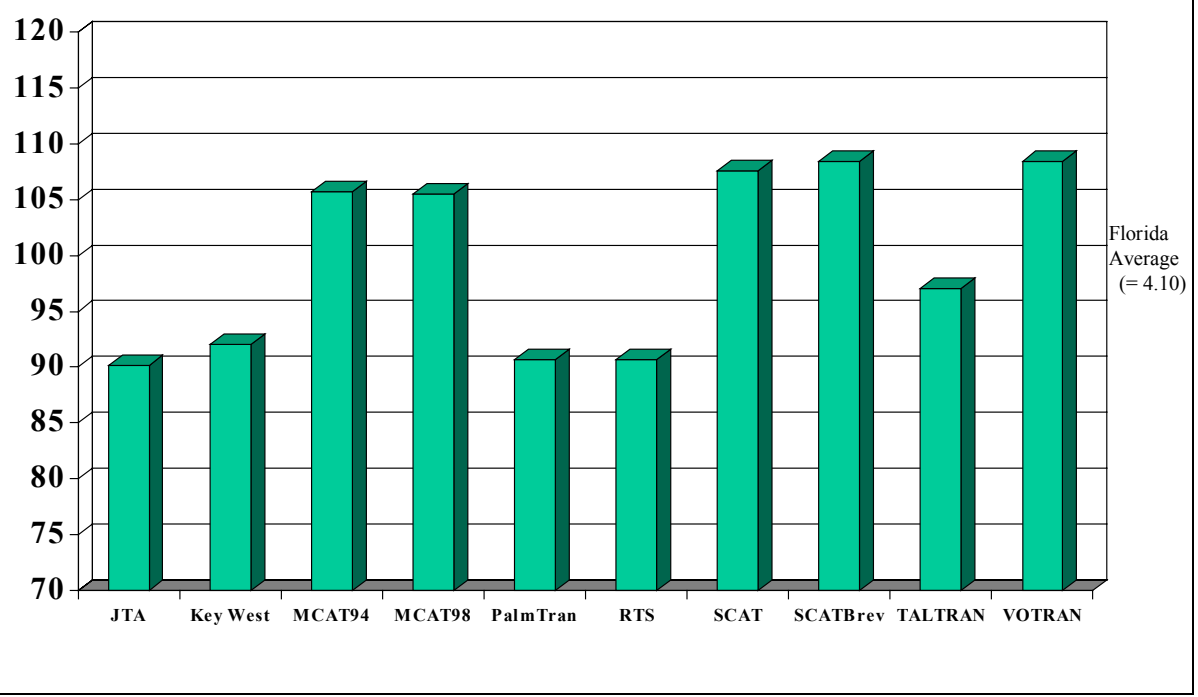

Figure 7: Ease of obtaining schedule 


\section{Transit Customer Satisfaction Index: Ease of using schedule}

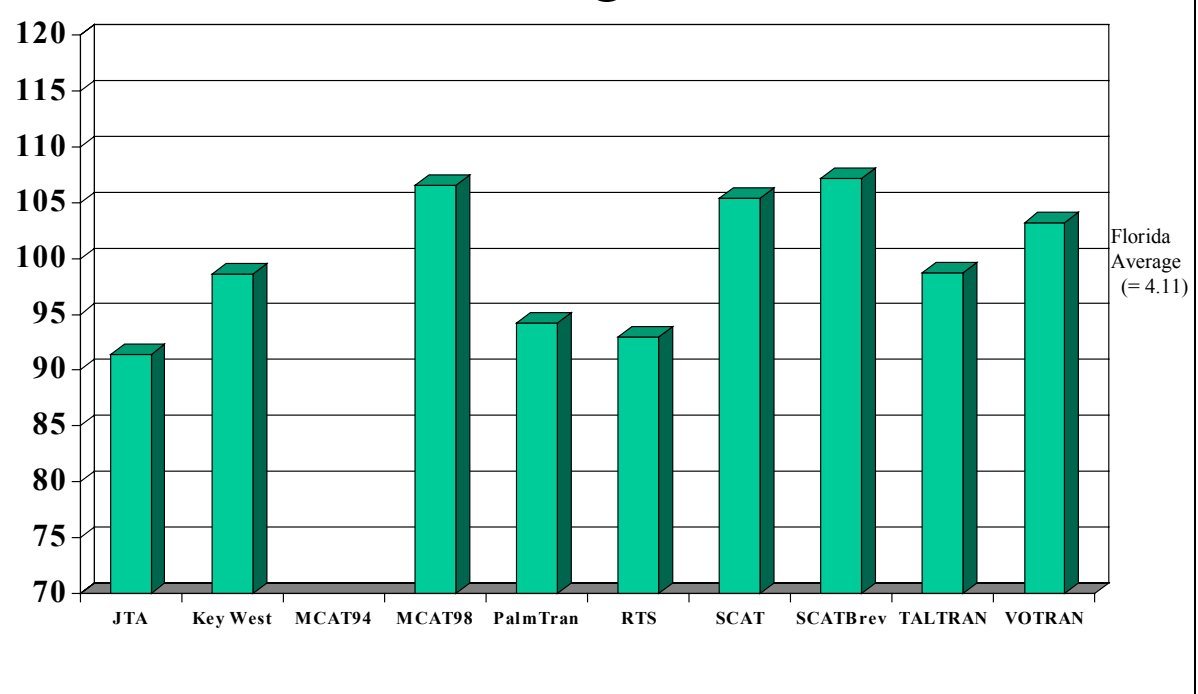

Figure 8: Ease of using schedule

\section{Transit Customer Satisfaction Index: Overall safety}

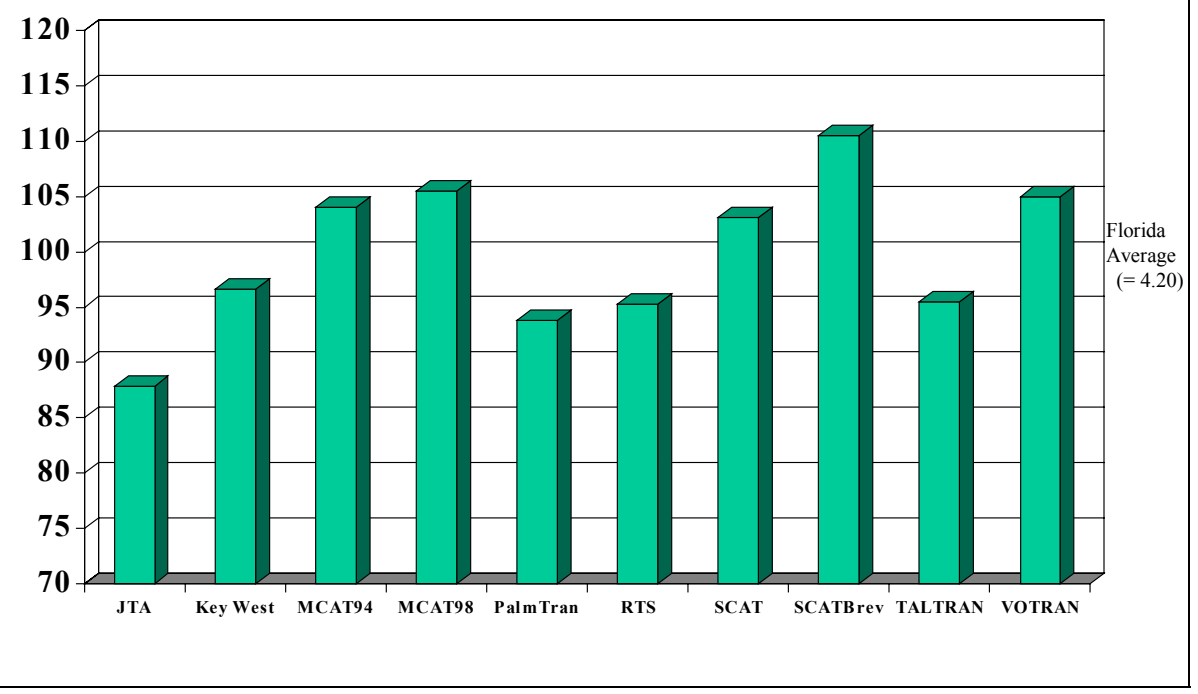

Figure 9: Overall safety 


\section{Transit Customer Satisfaction Index: Driver courtesy}

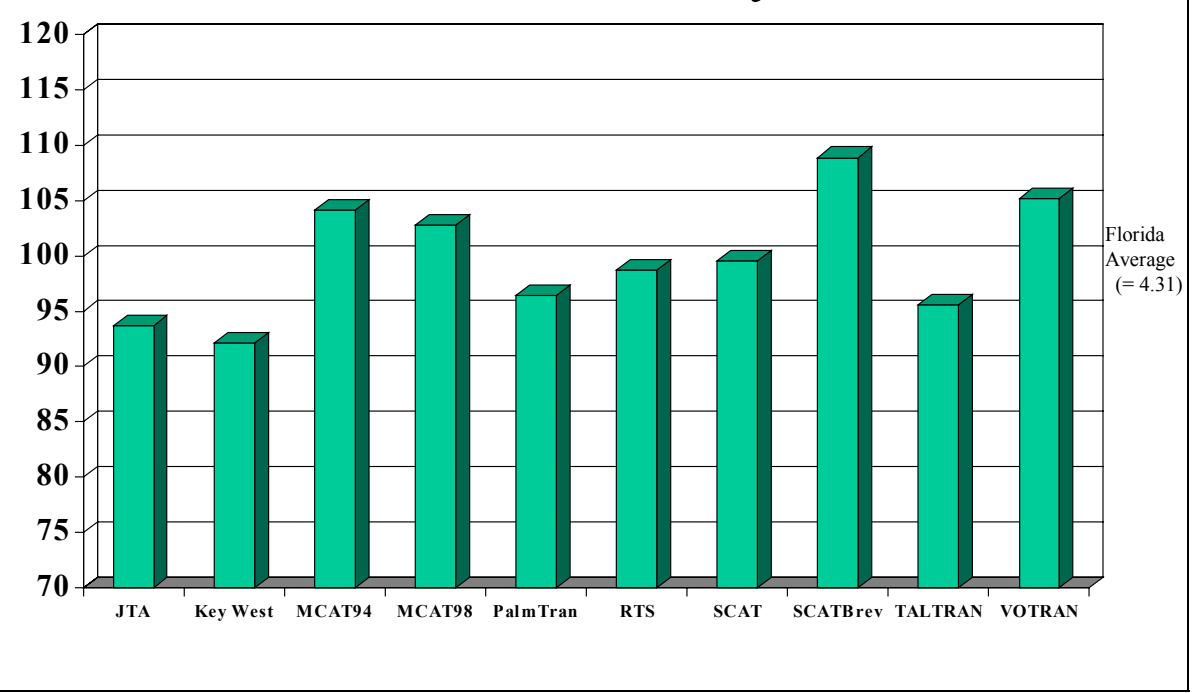

Figure 10: Driver courtesy

\section{Transit Customer Satisfaction Index: Convenience of routes}

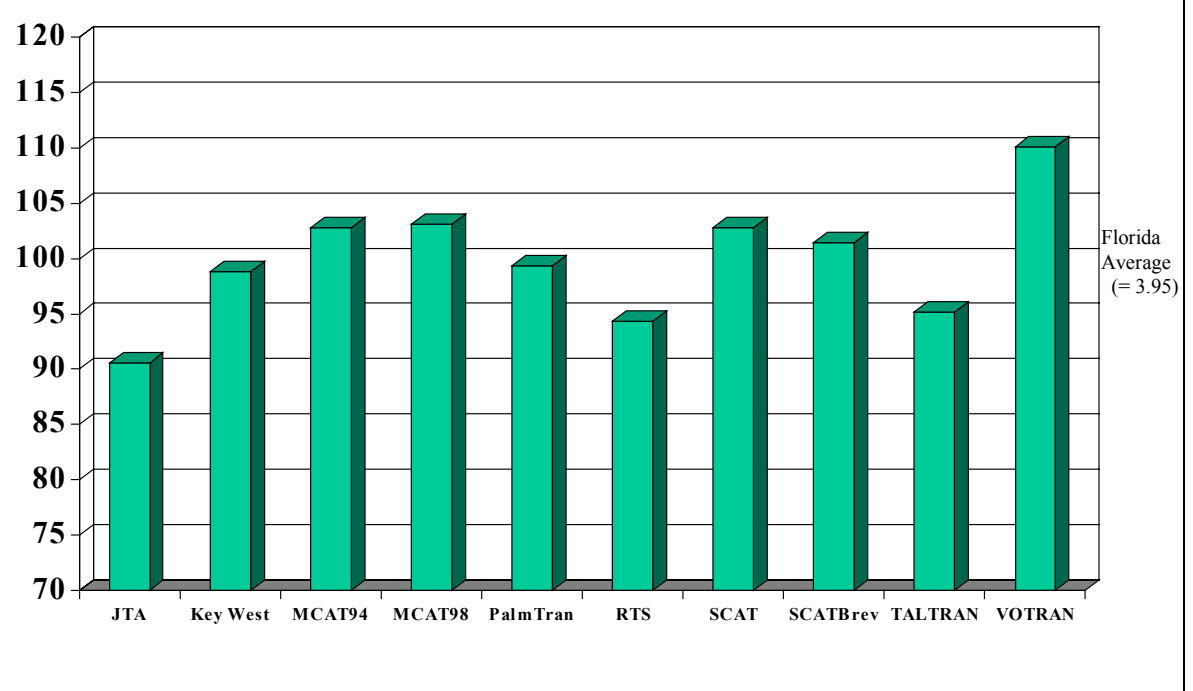

Figure 11: Convenience of routes 


\section{Transit Customer Satisfaction Index: Dependability of buses (on-time)}

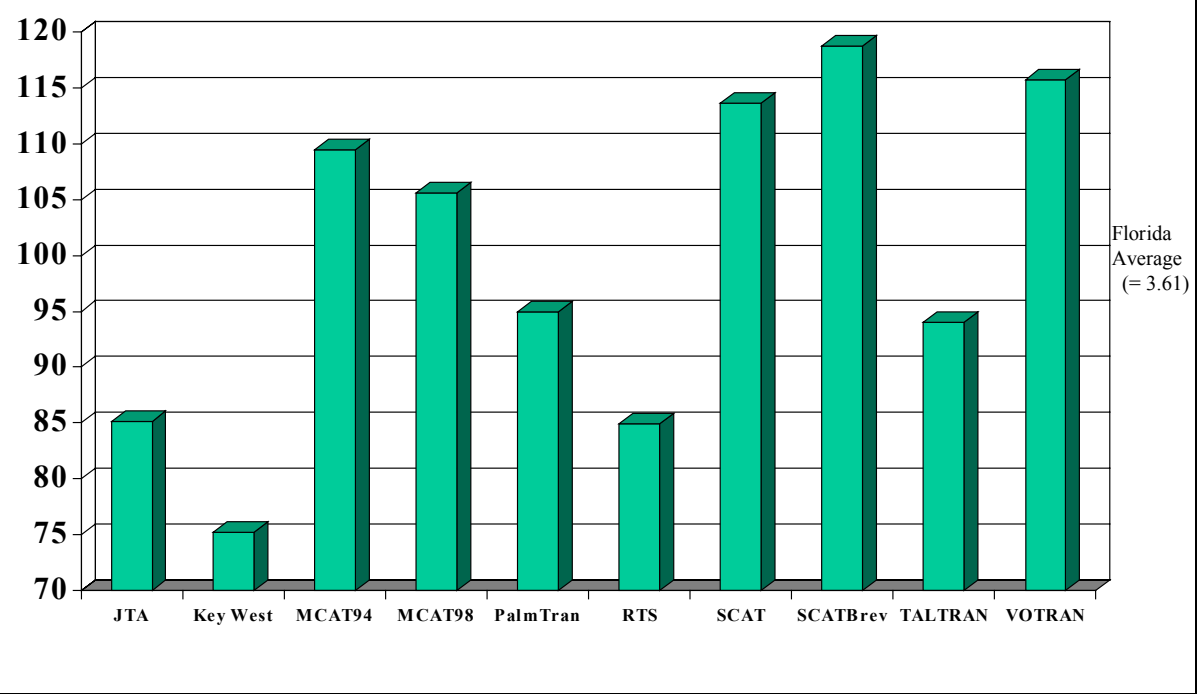

Figure 12: Dependability of buses (on time)

\section{Transit Customer Satisfaction Index:} Cleanliness \& Comfort

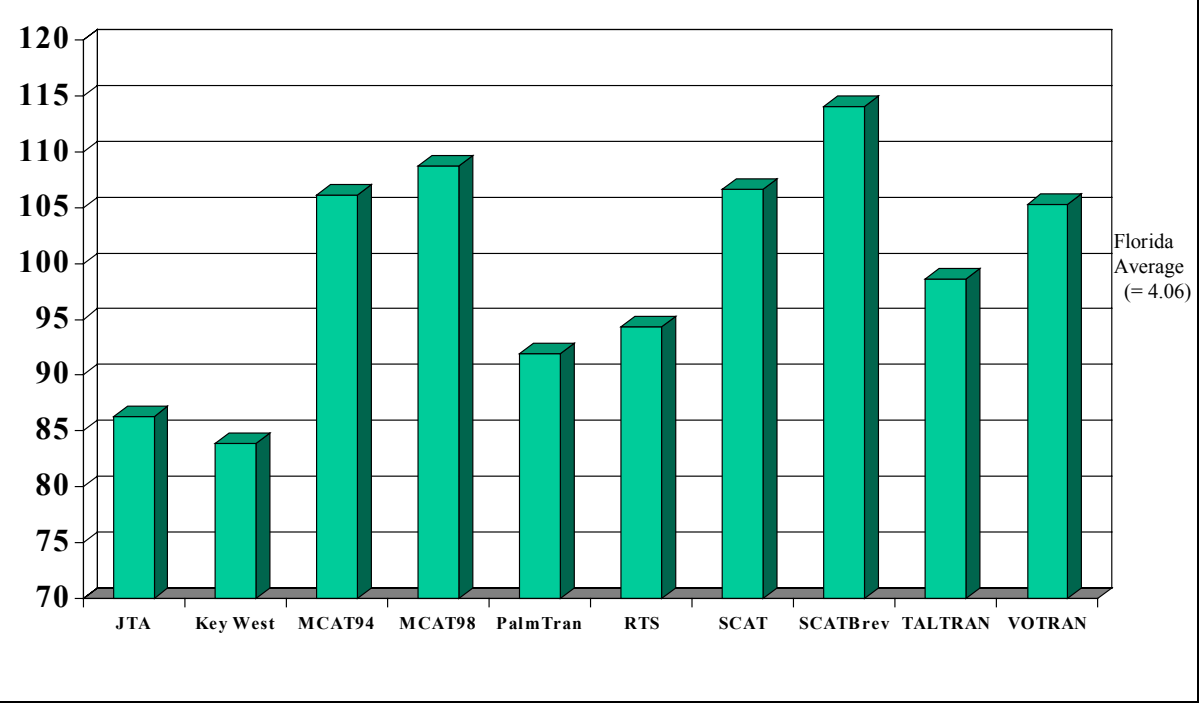

Figure 13: Cleanliness \& Comfort 
Since many of the surveys used for the 1999 project were similar to those used in 1996, it is possible to compare the overall Florida scores for these two years. A comparison chart appears below.

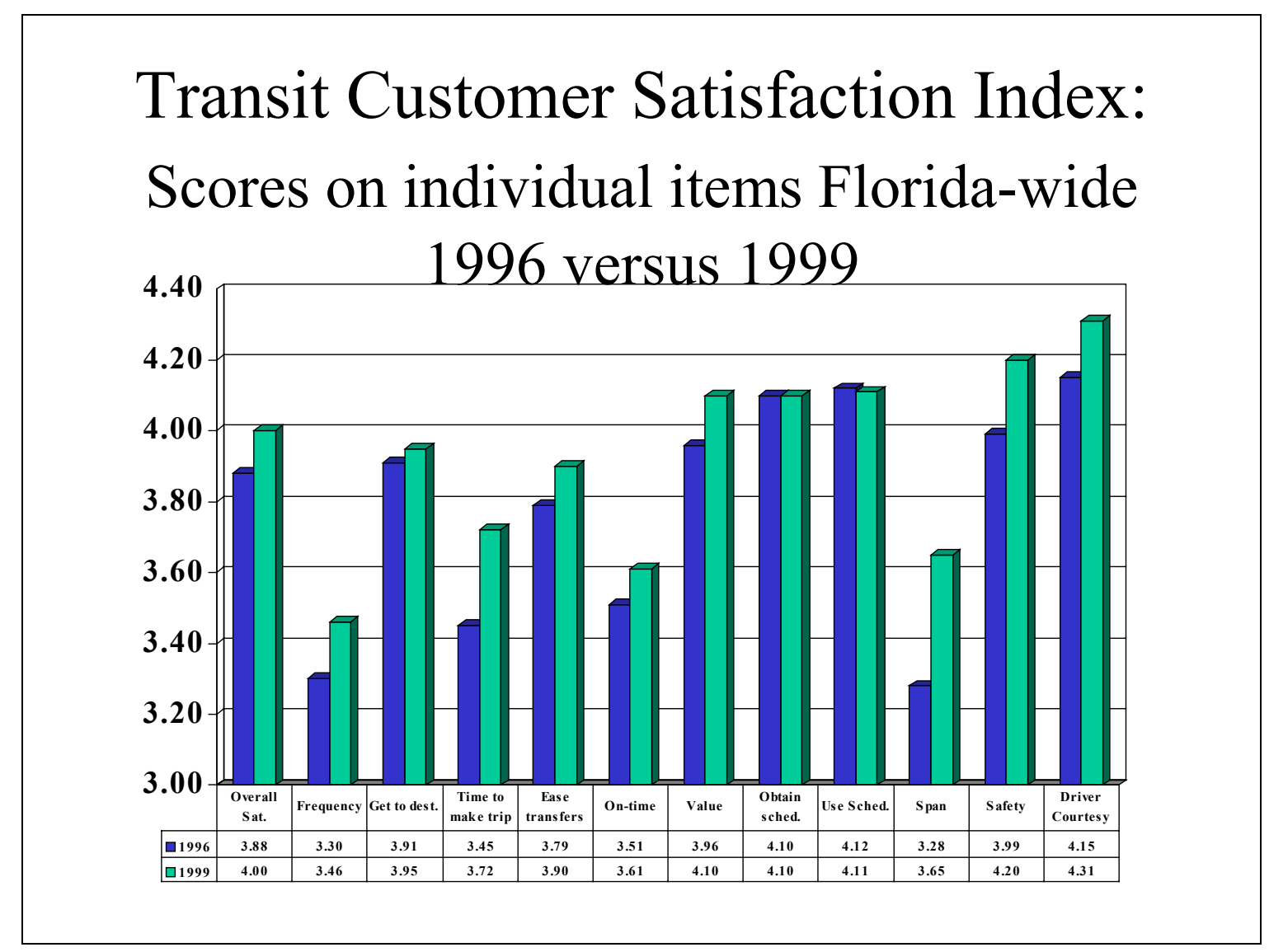

Figure 14: Scores on individual items Florida-wide 1996 versus 1999

While the examination of individual rating items may yield some interesting information, it is more informative to view these ratings in conjunction with a customer satisfaction model, which combines an "importance" rating of the items (derived from the regression model of satisfaction) to the customers with the performance rating provided directly by the respondents. 


\section{DEMOGRAPHICS}

In the next section, correlations are presented between relatively linear demographic variables (income, age, auto ownership) and satisfaction variables where relationships between the variables are reasonably linear. Next, group-to-group mean comparisons for non-linear demographic variables (ethnicity and gender) are presented where the differences are statistically significant and meaningful. For this report, a difference of 0.2 was considered a minimum difference to report the difference as meaningful.

\section{Income}

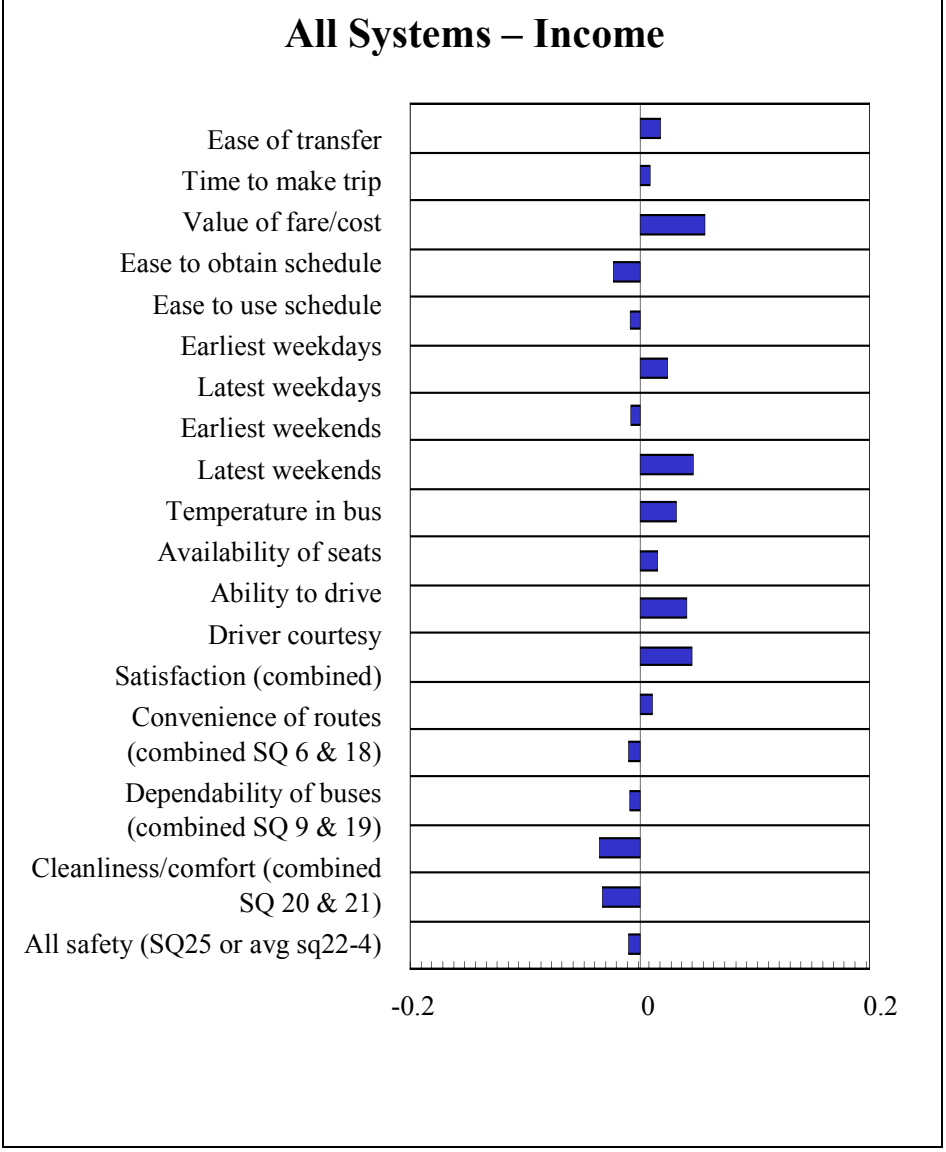

Figure 15: All Systems - Correlation of Income with satisfaction items

Analysis of a non-linearly related item, satisfaction with frequency of service, showed that riders with incomes of $\$ 25,000-\$ 50,000$ were significantly less satisfied than all 
other groups with frequency of service. Analysis of a second item, satisfaction with number of transfers, yielded the finding that riders with incomes of $\$ 50,000$ or more were significantly more satisfied with number of transfers required.

For the most part, higher income riders are more satisfied with transit service than lower income riders. This finding is likely due to the fact that higher income riders are more likely to be choice riders and hence will only use the bus when it is convenient for them. Thus those of higher income who are potential riders who feel that the bus is not convenient (in terms of routes, span of service, etc.) will choose not to ride the bus and would therefore not be sampled. Lower income riders do not have this choice.

The items with which lower income riders are more satisfied include ease of obtaining schedules, on-time arrivals, and cleanliness and comfort of buses. Higher frequency of riding the bus probably makes lower income riders more aware of where to obtain schedules. Lower income riders also probably use this familiarity to set more realistic expectations for these elements of the bus-riding than higher income riders. Thus the experiences that occur for which no prior expectation can reasonably be formed without prior use of the bus (such as on-time arrivals, cleanliness and comfort, temperature on the bus, availability of seats, and issues relating to the driver) may be expected to get higher ratings among lower-income users.

There are other mitigating factors to be considered. High-income riders are probably more satisfied with the temperature on the bus because the bus routes in the high-income neighborhoods may be less crowded. For similar reasons, satisfaction with availability of seats is higher among higher-income riders. The 'ability to drive' rating may be higher due to better maintenance (i.e. fewer potholes) of the roads used on these routes.

High-income patrons are more satisfied with safety at the bus stop, safety on the bus, and safety getting off bus because due to their higher income status they live in lower crime areas and are less likely to travel on the bus at night. Low-income riders rely on the bus more and may live in higher crime areas and must travel on the bus regardless of the time of day. They are, therefore, likely to be less satisfied with their safety on the bus. 


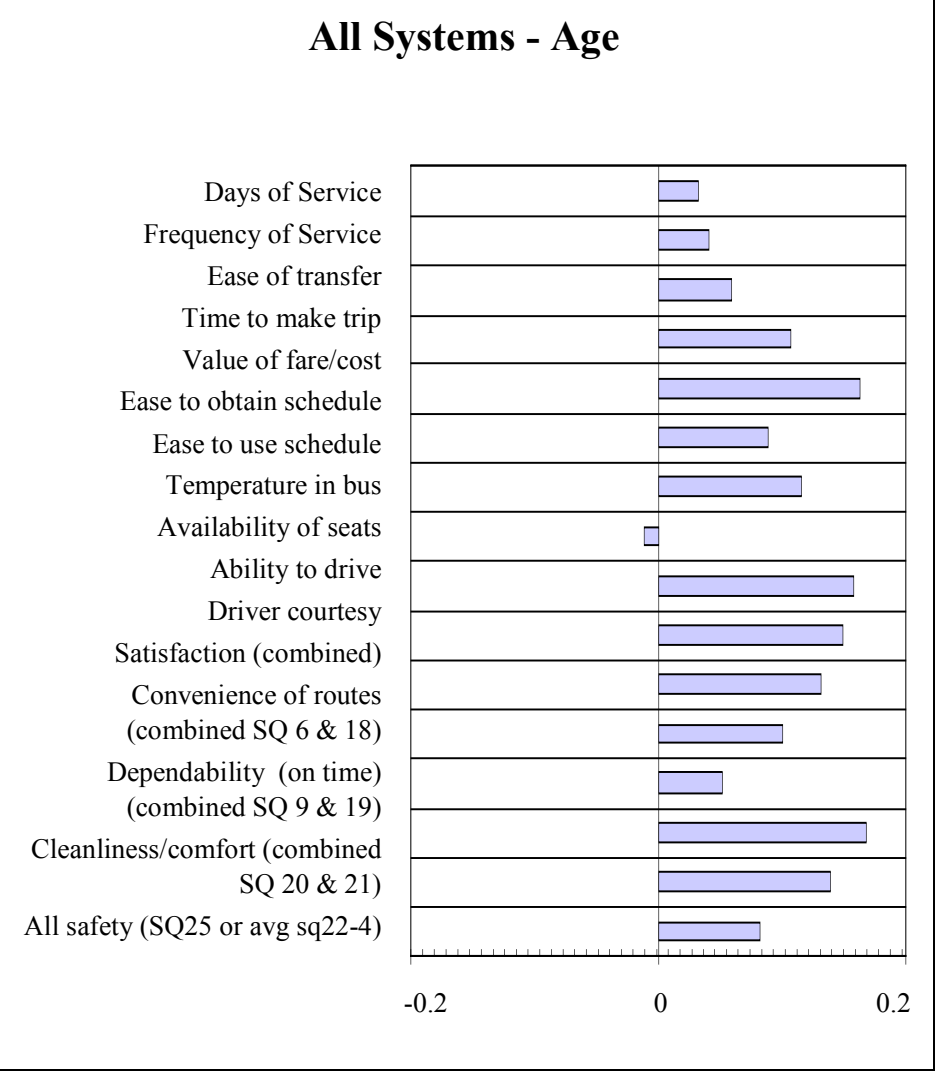

Figure 16: All Systems - Correlation of Age with satisfaction items

Analysis of a non-linearly related item, satisfaction with hours of service, showed riders who were under 17, and 60 and over, were more satisfied with hours of service provided, as well as with number of transfers.

Almost all of the satisfaction factors are positively correlated with age. Older respondents are overwhelmingly satisfied with transit service. As age increases, satisfaction increases for all but one category, temperature in bus. Since the negative correlation between age and temperature in bus appears to be statistically insignificant, an explanation is not appropriate. Different experiences between the older and younger individuals may cause the satisfaction differences between younger and older individuals. 
Individual time constraints and amount of leisure time can influence a passenger's satisfaction level. For instance, younger people are usually on a tighter schedule, such as arriving to work on time. Therefore, younger people will usually be more conscience of time delays. This can lead younger people to be less satisfied with frequency of service, ease of transfer, convenience of routes, and dependability. Also, younger passengers usually have less leisure time. Assuming that they would like to gain additional leisure time, their dissatisfaction with time to make trip is understandable. Moreover, younger riders tend to rate days of service with less satisfaction. Assuming that younger persons have less errand time during the weekday, they may perceive days of service to be dissatisfactory due to the fact that they need to take more trips on the weekend to fulfill all of their errands.

A combination of familiarity with surroundings as well as non-peak trips can lead to higher satisfaction ratings. Elderly people tend to utilize transit services in their particular neighborhood. Therefore, they are usually more familiar with their surroundings. Those who are more familiar with their surroundings tend to be generally more comfortable. Familiarity can lead older people to bestow higher ratings to driver courtesy, ability to drive, ease to obtain schedule, ease to use schedule, and safety factors. Riding a bus during non-peak trips increases the tendency of passengers to be more satisfied with the number of seats available on bus and cleanliness/comfort of bus. For example, a bus is perceived to be cleaner when there is less passenger congestion. Therefore, since older clients tend to ride the buses during non-peak service times, they tend to perceive the bus as being more clean/comfortable than younger passengers.

Finally, fare structure causes passengers to rate the value of transit differently. Many transit agencies offer discount fares to senior citizens. Hence, it is not surprising that the value of trip satisfaction increases with age. 


\section{$\underline{\text { Auto }}$}

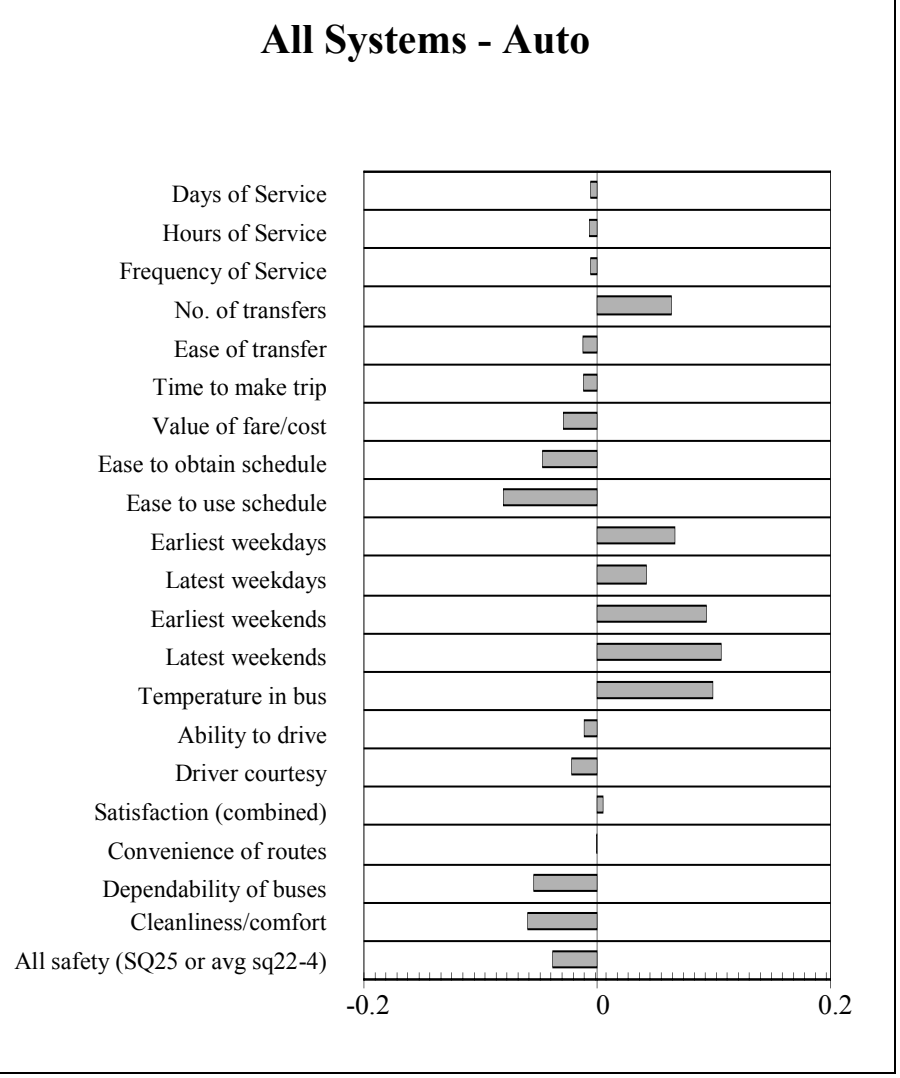

Figure 17: All Systems - Correlation of Auto ownership with satisfaction items

Analysis of a non-linearly related item, availability of seats, revealed that those who had two cars were more satisfied than those who had one car or three or more, but those who had 2 cars were not significantly more satisfied than those who had no cars.

Auto ownership impacts these satisfaction levels because there is a difference between riding buses due to convenience versus riding them out of necessity. A strong, positive correlation exists between number of automobiles and satisfaction with time service begins and ends for weekdays and weekends. Households that own automobiles have the luxury of 24-hour transportation. In contrast, households without vehicles are 
constrained by transit service times, taxi costs, and/or friends' schedules. Therefore, persons without automobiles are less satisfied with transit service begin and end times.

As car ownership increases, satisfaction for number of transfers increases. This positive relationship reveals a car owner's tendency to utilize transit for short trips. In contrast, those without cars rely on transit for all destinations. Usually, longer trips require more transfers, which can be seen as cumbersome by those who use transit as their only reliable form of transportation.

Satisfaction of the value of fare/cost is negatively correlated with number of automobiles in household. Those without cars perceive transit as more valuable because they utilize it for all types of trips. Without transit, these passengers would not be able to work, go to school, run errands, and/or utilize public facilities. In contrast, customers who own automobiles tend to underestimate the value of transit. Automobile owners do not know the difficulties associated with trying to obtain rides for errands when transit is not available.

Familiarity with transit service may also cause negative correlations between automobile ownership and transit satisfaction. For instance, ease to obtain schedule, ease to use schedule, ease of transfer, driver courtesy, dependability of buses and safety are all issues that are negatively correlated with ownership. Much can be explained by the fact that those who do not have cars use transit the most. Therefore, familiarity with bus schedules and bus routes will lead persons without cars to be more satisfied. A negative correlation between ease of transfer and ownership suggests that since the non-owners frequently depend upon bus transfers, they are used to the routine method and therefore perceive it as easier. Those who use transit often will also be more familiar with bus drivers and bus conditions, thereby inciting a higher satisfaction with driver courtesy and safety. Also, the riders who use transit the most will have a more realistic view of bus dependability and allow for more time to make trip.

Automobile owners may have extremely high expectations of transit service. For instance, owners tend to be less satisfied with the bus driver's ability to drive. A likely explanation is that automobile owners are used to driving and controlling a vehicle's whereabouts. Therefore, owners feel out of control when their rides depend on a complete stranger's driving abilities. Finally, lower income passengers perceived bus 
cleanliness and comfort to be more satisfactory than higher income passengers. This suggests that the riders who own automobiles are used to choosing their passengers. Auto owners may not be as comfortable with their fellow transit clientele and may be especially uncomfortable on crowded buses.

Virtually no correlation exists between number of household automobiles and days of service, hours of service, frequency of service, and convenience of routes. Therefore, no explanation is given. Similarly, the strong positive correlation between number of automobiles in household and temperature in bus is difficult to explain. A crossreference of low income/high auto households (across all systems, to allow sufficient sample size) shows that their level of satisfaction with temperature on the bus is marginally higher than those with low incomes and fewer vehicles (3.90 to 3.80). Those with high incomes and 3 or more autos have average ratings of 4.17. This finding may relate to the presence of vehicles with air conditioning in the household, i.e. that those who have vehicles with air conditioning find bus temperature comfortable, but perhaps others find it too cold. 
Below are the comparisons for correlations for non-linear demographics (i.e. race and gender) and satisfaction items. Only those satisfaction items where a significant difference between groups was found are presented.

All the questions in listed in order of largest differences, from those where the right hand column (for example, "Black" in the first table below) has the largest positive difference from the left hand column ("White" in the table below) to those where the left hand column has the largest positive difference over the right hand column. Thus, in the first table below, the first listing is "Latest weekdays" where blacks have a higher average score than whites by 0.22 , and the last listing is satisfaction with dependability of buses (on-time), where whites have a higher average score than blacks by 0.47 .

It should be noted that any of the differences observed can have an adequate, reasonable, and sound explanation for being higher or lower for either group. For instance, whites could be more satisfied than blacks with bus service because they are choice riders and only use the bus when it is completely convenient and thus don't use it when it isn't satisfactory, and hence aren't surveyed; or , blacks could be more satisfied than whites because through having more experience riding the bus, know their way around the system and how to use it, and have more realistic expectations of what bus service can and will provide. Both explanations could be correct. The one that fits the data is chosen because it is assumed that it must be predominantly correct for the system in question. 
Non-linear demographic items - Ethnicity

\begin{tabular}{|c|c|c|c|}
\hline & Table 4-I: All Systems Ethnicity & White & Black \\
\hline Sample size & & $\mathbf{6 3 0 8}$ & $\mathbf{6 5 7 1}$ \\
\hline & & & \\
\hline SQ15 & Latest weekdays & 3.03 & 3.25 \\
\hline SQ31 & Satisfaction (combined) & 4.10 & 3.92 \\
\hline SQ07 & No. of transfers & 3.69 & 3.51 \\
\hline SQ14 & Earliest weekdays & 3.83 & 3.64 \\
\hline SQ29 & Driver courtesy & 4.42 & 4.21 \\
\hline SQ10 & Time to make trip & 3.84 & 3.60 \\
\hline SQ25 & Safety on bus \& stops & 4.45 & 4.18 \\
\hline SQ34 & Cleanliness/comfort (combined SQ 20 \& 21) & 4.20 & 3.92 \\
\hline SQ11 & Value of fare/cost & 4.22 & 3.94 \\
\hline SQ33 & Dependability of buses (on time) (combined SQ 9 \& 19) & 3.78 & 3.44 \\
\hline
\end{tabular}

In most cases, whites have higher levels of satisfaction than blacks. This is probably related to many of the same factors that create higher satisfaction levels among higher income riders - mainly because they can be choice riders and are more apt to use the transit when it is convenient, but always having the option to drive where transit service does not meet their need for convenience and speed.

On only one item did blacks have higher ratings than whites. That item was 'Satisfaction with latest weekday service,' which for both groups and in total was the lowest rated item in the survey. This is somewhat surprising, since it is expected that whites are more likely to be choice riders and presumably would not be as affected by service end times. The finding may either be a random aberration or it may be that the earlier end times for service in areas predominantly populated by whites affects white riders so much that the impact of having more choice rider in that population segment is overwhelmed. 


\begin{tabular}{|c|c|c|c|}
\hline & Table 4-II: All Systems Ethnicity & White & Hispanic \\
\hline Sample size & & $\mathbf{6 3 0 8}$ & $\mathbf{9 5 9}$ \\
\hline & & & \\
\hline SQ17 & Latest weekends & 3.09 & 3.42 \\
\hline SQ16 & Earliest weekends & 3.52 & 3.72 \\
\hline SQ34 & Cleanliness/comfort (combined SQ 20 \& 21) & 4.20 & 4.00 \\
\hline SQ33 & Dependability of buses (on time) (combined SQ 9 \& 19) & 3.78 & 3.47 \\
\hline
\end{tabular}

Hispanic riders are more satisfied than whites with times of weekend service. They are less satisfied with cleanliness/comfort and dependability of service. This seems to imply that Hispanics are more dependent on the bus during the week but less so on the weekends.

\begin{tabular}{|c|c|c|c|}
\hline & Table 4-III: All Systems Ethnicity & Black & Hispanic \\
\hline Sample size & & $\mathbf{6 5 7 1}$ & $\mathbf{9 5 9}$ \\
\hline & & & 3.83 \\
\hline SQ07 & No. of transfers & 3.51 & 3.72 \\
\hline SQ16 & Earliest weekends & 3.41 & 3.42 \\
\hline SQ17 & Latest weekends & 3.12 & 4.47 \\
\hline SQ25 & Safety on bus \& stops & 4.18 & 4.25 \\
\hline SQ24 & Safety getting off bus & 3.97 & 4.11 \\
\hline SQ22 & Safety at bus stop & 3.84 & 4.19 \\
\hline SQ11 & Value of fare/cost & 3.94 & 3.87 \\
\hline SQ14 & Earliest weekdays & 3.64 & 4.06 \\
\hline SQ08 & Ease of transfer & 3.85 & \\
\hline
\end{tabular}

Differences between blacks and Hispanics essentially mirror those between whites and Hispanics, since there are so few differences between white and Hispanic satisfaction levels. This analysis would assume that Hispanics have slightly higher levels of choice ridership than blacks. It is also interesting to note that satisfaction with number of and ease of transfers is higher among Hispanics, suggesting that they tend to live in neighborhoods with more direct routes to their destinations.

There are no items for which blacks are significantly more satisfied than Hispanics. 


\begin{tabular}{|c|c|c|c|}
\hline & Table 4-IV: All Systems Ethnicity & White & Other \\
\hline Sample size & & $\mathbf{6 3 0 8}$ & $\mathbf{9 0 5}$ \\
\hline & & & \\
\hline SQ10 & Time to make trip & 3.84 & 3.65 \\
\hline SQ14 & Earliest weekdays & 3.83 & 3.64 \\
\hline SQ07 & No. of transfers & 3.69 & 3.50 \\
\hline SQ08 & Ease of transfer & 3.96 & 3.74 \\
\hline SQ25 & Safety on bus \& stops & 4.45 & 4.22 \\
\hline SQ03 & Days of Service & 4.16 & 3.91 \\
\hline SQ11 & Value of fare/cost & 4.22 & 3.97 \\
\hline SQ22 & Safety at bus stop & 3.99 & 3.73 \\
\hline SQ05 & Frequency of Service & 3.48 & 3.22 \\
\hline SQ17 & Latest weekends & 3.09 & 2.81 \\
\hline SQ13 & Ease to use schedule & 4.16 & 3.88 \\
\hline SQ04 & Hours of Service & 3.69 & 3.39 \\
\hline SQ31 & Satisfaction (combined) & 4.10 & 3.79 \\
\hline SQ29 & Driver courtesy & 4.42 & 4.11 \\
\hline SQ32 & Convenience of routes (combined SQ 6 \& 18) & 4.02 & 3.70 \\
\hline SQ26 & Temperature in bus & 3.92 & 3.51 \\
\hline SQ34 & Cleanliness/comfort (combined SQ 20 \& 21) & 4.20 & 3.80 \\
\hline SQ33 & Dependability of buses (on time) (combined SQ 9 \& 19) & 3.78 & 3.33 \\
\hline SQ16 & Earliest weekends & 3.52 & 3.00 \\
\hline
\end{tabular}

\begin{tabular}{|c|c|c|c|}
\hline & Table 4-V: All Systems Ethnicity & Black & Other \\
\hline Sample size & & $\mathbf{6 5 7 1}$ & $\mathbf{9 0 5}$ \\
\hline & & & \\
\hline SQ04 & Hours of Service & 3.59 & 3.39 \\
\hline SQ13 & Ease to use schedule & 4.08 & 3.88 \\
\hline SQ32 & Convenience of routes (combined SQ 6 \& 18) & 3.91 & 3.70 \\
\hline SQ03 & Days of Service & 4.17 & 3.91 \\
\hline SQ05 & Frequency of Service & 3.49 & 3.22 \\
\hline SQ26 & Temperature in bus & 3.81 & 3.51 \\
\hline SQ17 & Latest weekends & 3.12 & 2.81 \\
\hline SQ15 & Latest weekdays & 3.25 & 2.87 \\
\hline SQ16 & Earliest weekends & 3.41 & 3.00 \\
\hline
\end{tabular}




\begin{tabular}{|c|c|c|c|}
\hline & Table 4-VI: All Systems Ethnicity & Hispanic & Other \\
\hline Sample size & & $\mathbf{9 5 9}$ & $\mathbf{9 0 5}$ \\
\hline & & & \\
\hline SQ24 & Safety getting off bus & 4.25 & 4.07 \\
\hline SQ29 & Driver courtesy & 4.30 & 4.11 \\
\hline SQ04 & Hours of Service & 3.59 & 3.39 \\
\hline SQ34 & Cleanliness/comfort (combined SQ 20 \& 21) & 4.00 & 3.80 \\
\hline SQ31 & Satisfaction (combined) & 4.00 & 3.79 \\
\hline SQ11 & Value of fare/cost & 4.19 & 3.97 \\
\hline SQ14 & Earliest weekdays & 3.87 & 3.64 \\
\hline SQ25 & Safety on bus \& stops & 4.47 & 4.22 \\
\hline SQ32 & Convenience of routes (combined SQ 6 \& 18) & 3.99 & 3.70 \\
\hline SQ13 & Ease to use schedule & 4.18 & 3.88 \\
\hline SQ08 & Ease of transfer & 4.06 & 3.74 \\
\hline SQ07 & No. of transfers & 3.83 & 3.50 \\
\hline SQ15 & Latest weekdays & 3.22 & 2.87 \\
\hline SQ22 & Safety at bus stop & 4.11 & 3.73 \\
\hline SQ26 & Temperature in bus & 3.98 & 3.51 \\
\hline SQ17 & Latest weekends & 3.42 & 2.81 \\
\hline SQ16 & Earliest weekends & 3.00 \\
\hline
\end{tabular}

Members of 'Other' races, probably mainly Asians and native Americans, are significantly less satisfied with almost all elements of bus service than whites, Hispanics, and blacks. Overall satisfaction levels are particularly low. This may be partially a cultural phenomenon (i.e., a tendency to give lower ratings on surveys) but may also reflect lower level of service (i.e., less frequency, and later start / earlier end times) to neighborhoods predominantly populated by these racial groups.

\section{Non-linear Demographic Items: Gender}

There were no significant differences between levels of satisfaction between females and males. Differences occurred between minority groups rather than along gender lines. 


\section{CUSTOMER SATISFACTION MODELING}

A simple linear regression was built to explain the overall satisfaction ratings in terms of the ratings.

Since many of the independent variables were intercorrelated, there was a high probability that the coefficients resulting from model runs would not reflect the effects of each of the independent variables. The standard approach to eliminating the effects of the multicollinearity is to run an initial factor analysis.

The analytical procedure of factor analysis involves creating uncorrelated (orthogonal) combinations of the initial independent variables. The purpose of the analysis is to reduce a mass of variables to a reasonable number of elements that the analyst can understand and explain. Often, the selection of factors to use is limited to those that explain at least as much variance as an independent variable, i.e., the output factor has an eigenvalue of at least one. However, this approach is more of a guideline for the purposes of efficiency than a required analytical rule. In fact, some factor analysts even maintain that any factor with a positive eigenvalue is relevant for analysis. In this application, the factor structures were examined to determine which factors provided suitable and explainable combinations of variables, and those factors were used as independent predictor variables for the overall satisfaction rating.

The factors are represented as combinations of the independent data elements; for example, the Safety factor combines Safety at bus stops, on buses, and after getting off the bus, and the Span of Service factor combines satisfaction with earliest and latest departure times on weekdays and weekends. A score for a factor can be calculated in two ways - either by using the variable loadings on each factor (essentially creating a weighted score) or by taking a simple average of the variables that load primarily onto the factor. The latter approach is both computationally simpler and easier to understand, so it will be applied in this instance.

For both the factor modeling and the satisfaction modeling, a mean substitution procedure was used for those respondents who had not filled out every item in order to allow their input into the models. 
The factor structures were created independently for each of the systems, since it was considered likely that each system's riders may have a different view of their system's operations. The same argument could be made for different demographic groups (male vs. female, income levels, etc.) or other potential rider classifications (such as frequent vs. infrequent users, and so forth), but from a system operations point of view, where any changes made will likely affect the entire system, the most logical modeling process is to treat the system's riders as a homogeneous whole. A route-by-route assessment might also make sense if such detailed data were available in sufficient quantity, but this is not the case in this study.

The model was built by using the true factor scores, which involve multiplying each of the independent variables by its respective loading coefficient for the factor in question. The actual factor score is not precisely equal to the mean of the main loading variables, but this simplification will not be misleading and is much easier to understand and act upon. Where respondents had not answered some of the individual satisfaction items but had provided a response to the overall satisfaction question, a mean-substitution procedure was used to bolster the sample available for analysis.

A total of twelve factors were identified. The composition of these factors varied from system to system, but these twelve constructs effectively describe how Florida transit customers think about transit service.

Because each system's factor structure and resulting customer satisfaction model is unique, they will each be examined and discussed separately. 


\section{INDIVIDUAL SYSTEM RESULTS}

What follows are the results for each of the individual systems, analyzed in detail. The information is presented in the following format:

- $\quad$ Factor analysis and results by factor

Each of the factors is shown together with the index and mean absolute scores for each item in the factor. The factors have been numbered so that the numbering is consistent across different systems. Thus Factor 2 in TALTRAN is essentially the same as Factor 2 for JTA. Since not all factors are used for all systems, this necessarily means that factors will not be sequentially numbered within a system. Therefore, you may have a system with Factor 1-4, Factor 8, and Factor 12 rather than simply Factors 1-6.

- $\quad$ Satisfaction model

The regression modeled developed to predict overall satisfaction from the factor scores is presented. Importance of each of the service factors is developed from the regression model

- Demographic analysis

- Linear variables: correlation with relatively linear demographic variables (income, age, auto ownership) is presented.

○ Non-linear variables: Group-to-group mean comparisons for non-linear demographic variables (ethnicity and gender) are presented where the differences are statistically significant and meaningful. For this report, a difference of 0.2 was considered a minimum difference to report the difference as meaningful. It should be noted that any of the differences observed can have an adequate, reasonable, and sound explanation for being higher or lower for either group. For instance, whites could be more satisfied than blacks with bus service because they are choice riders and only use the bus when it is completely convenient and thus don't use it when it isn't satisfactory, and hence aren't surveyed; or, blacks could be more satisfied than whites because through having more experience riding the bus, know their way around the system and how to use it, and have more realistic expectations of what bus service can and will provide. Both explanations could be correct. The one that fits the data is chosen because 
it is assumed that it must be predominantly correct for the system in question.

- $\underline{\text { Recommendations }}$

Recommendations for each system arising from the demographic analysis and the regression model are presented. 


\section{Key West}

$\underline{\text { Factor Analysis }}$

Factor 1 - $\quad$ System Design

Major loadings - $\quad$ SQ5 Frequency of service

SQ10 Time to make trip

SQ9 How regularly buses arrive on time

SQ6 Ability to get where you want to go

This construct relates to customer satisfaction with system design that includes frequency of service, time to make trip, how regularly buses arrive on time, and ability to get where you want to go. It is important to note that the variables related to making transfers did not load onto this factor as expected but instead constitute an entire factor on their own.

\begin{tabular}{|c|c|c|}
\hline \multirow[t]{3}{*}{ Table 5-I: Key West Facto } & System & \\
\hline & \multicolumn{2}{|c|}{ Scores } \\
\hline & Index & Mean \\
\hline Frequency of Service & 90.19 & 3.12 \\
\hline Time to Make Trip & 92.67 & 3.45 \\
\hline How regularly Buses Arrive On Time & 82.18 & 2.72 \\
\hline Ability to Get Where You Need To Go & 99.76 & 3.90 \\
\hline Overall Mean & & 3.30 \\
\hline
\end{tabular}


Factor $2-$ Span of Service

Major loadings - $\quad$ SQ15 Latest weekdays

SQ17 Latest weekends

SQ16 Earliest weekends

SQ14 Earliest weekdays

This construct relates to customer satisfaction with span of transit service availability.

The components are satisfaction with earliest weekday, latest weekday, earliest weekend day, and latest weekend day service.

\begin{tabular}{|lc|c|}
\hline \multicolumn{2}{|c|}{ Table 5-II: Key West Factor 2 - Span of Service } \\
\cline { 2 - 3 } & \multicolumn{2}{c|}{ Scores } \\
\cline { 2 - 3 } & Index & Mean \\
\hline Latest weekdays & 106.78 & 3.32 \\
\hline Latest weekends & 103.03 & 3.20 \\
\hline Earliest weekends & 107.70 & 3.72 \\
\hline Earliest weekdays & 101.59 & 3.81 \\
\hline \multirow{2}{*}{ Overall Mean } & & 3.51 \\
\hline
\end{tabular}


Factor 3 - Perceptions of Safety

Major loadings - $\quad$ SQ28 Ability to drive

SQ29 Driver courtesy

SQ23 Safety on bus

SQ24 Safety getting off bus

Minor loadings - $\quad$ SQ6 Ability to get where you want to go

SQ22 Safety at bus stop

This construct relates to customers' perception of safety. The driver's ability to drive, passenger safety at the bus stop, and passenger safety after getting off the bus are obvious safety concerns. It seems reasonable to conclude that the driver courtesy rating is included in this construct because high levels of driver courtesy leads customers to believe that the driver, as the representative of the system, cares about them and will watch for their physical well being i.e. safety.

\begin{tabular}{|lc|c|}
\hline \multicolumn{2}{|c|}{ Table 5-III: Key West Factor 3 - Perceptions of Safety } \\
\cline { 2 - 3 } & \multicolumn{2}{c|}{ Scores } \\
\cline { 2 - 3 } Item & Index & Mean \\
\hline Ability to drive & 98.32 & 4.21 \\
\hline Driver courtesy & 92.15 & 3.97 \\
\hline Safety on bus & 99.20 & 4.11 \\
\hline Safety getting off bus & 101.61 & 4.12 \\
\hline Ability to get where you want to go & 99.76 & 3.90 \\
\hline Safety at bus stop & 101.01 & 3.96 \\
\hline Overall Mean & & 4.11 \\
\hline
\end{tabular}




\section{Factor 4 - $\underline{\text { Transfers }}$}

Major loadings - $\quad$ SQ7 Number of transfers

SQ8 Ease of transfer

The construct here is customer experience with transfer, related both to the customers' experience of the ease of transfers between buses as well as the number of transfers required to complete their trip.

\begin{tabular}{|lc|c|}
\hline \multicolumn{2}{|c|}{ Table 5-IV: Key West Factor 4 - Transfers } \\
\cline { 2 - 3 } & \multicolumn{2}{c|}{ Scores } \\
\cline { 2 - 3 } \multicolumn{1}{c|}{ Item } & Index & Mean \\
\hline Number of Transfers & 102.28 & 3.70 \\
\hline Ease of Transfers & 101.11 & 3.94 \\
\hline \multirow{2}{*}{ Overall Mean } & 3.82 \\
\hline
\end{tabular}


Factor 5 - $\underline{\text { Value }}$

Major loadings - $\quad$ SQ12 Ease to obtain schedule

SQ11 Value of fare/cost

This construct is strongly related to customer satisfaction with the value of the fare.

Interestingly, ease of obtaining schedules also loads strongly on to this factor. This

indicates that customers' perception of value is highly related to their ability to obtain a schedule. As a result, it may be advantageous to make schedules more easily accessible to customers.

\begin{tabular}{|lc|c|}
\hline \multicolumn{3}{|c|}{ Table 5-V: Key West Factor 5 - Value } \\
\hline \multirow{2}{*}{ Item } & Index & Mean \\
\cline { 2 - 3 } & 92.05 & 3.77 \\
\hline Ease to Obtain Schedule & 105.06 & 4.31 \\
\hline Value of Fare/Cost & $\mathbf{4 . 1 8}$ \\
\hline \multirow{2}{*}{ Overall Mean } & \\
\hline
\end{tabular}


Factor 6 - $\quad$ Comfort of Ride

Major loadings - $\quad$ SQ27 Availability of buses

SQ26 Temperature in bus

Minor loadings - $\quad$ SQ10 Time to make trip

SQ14 Earliest weekdays

SQ16 Earliest weekends

This construct relates to customer satisfaction with the availability of seats and temperature in bus. Other variables slightly loading on this factor are the time to make trip, earliest weekday service, and earliest weekend service. This indicates that customers who would prefer earlier service are generally not satisfied with availability of seats and bus temperature - perhaps they feel it has gotten too hot to ride comfortably by the time the first bus of the day arrives.

\begin{tabular}{|c|c|c|}
\hline \multirow[t]{3}{*}{ Table 5-VI: } & Comfol & \\
\hline & \multicolumn{2}{|c|}{ Scores } \\
\hline & Index & Mean \\
\hline Availability of Seats & 96.95 & 3.78 \\
\hline Temperature in Bus & 91.56 & 3.52 \\
\hline Time to make Trip & 92.67 & 3.45 \\
\hline Earliest Weekdays & 101.59 & 3.81 \\
\hline Earliest Weekends & 107.70 & 3.72 \\
\hline Overall Mean & & 3.65 \\
\hline
\end{tabular}


Factor 7 - $\quad \underline{\text { Schedule }}$

Major loading - $\quad$ SQ13 Ease to use schedule

Minor loadings - $\quad$ SQ12 Ease to obtain schedule

SQ9 How regularly buses arrive on time

The construct relates to customer experience using schedules. The main variable in this construct is satisfaction with the ease of using the schedule. Predictably, other variables loading on this factor are ease to obtain schedule and how regularly the buses arrive on time.

\begin{tabular}{|c|c|c|}
\hline \multirow[t]{3}{*}{ le 5-VII: Key Wes } & $7-\mathrm{Scl}$ & \\
\hline & \multicolumn{2}{|c|}{ Scores } \\
\hline & Index & Mean \\
\hline Ease to Use Schedule & 98.58 & 4.05 \\
\hline Ease to Obtain Schedule & 92.05 & 3.77 \\
\hline How regularly buses arrive on time & 82.18 & 2.72 \\
\hline Overall Mean & & 3.51 \\
\hline
\end{tabular}




\section{Factor 12 - Cleanliness/Safety}

Major loadings - $\quad$ SQ20 Clean buses and stop

SQ22 Safety at bus stop

Minor loadings - $\quad$ SQ23 Safety on bus

SQ24 Safety getting off bus

This construct relates to customer satisfaction with clean buses and stops as well as safety at bus stop. Other variables slightly loading on this factor include temperature in the bus, safety getting off bus, and safety on bus and stops. The connection between safety at bus stops and cleanliness requires some explanation. One possibility is that the passenger construct relates to the apparent level of concern and respect the transit agency has for their passengers. Cleanliness is a very common way of showing respect and concern for customers - in restaurants, retail businesses, even in personal interactions. One of the correlates of transit agency concern for customers could be safety at bus stops, since the perception that the agency is taking care of its facilities may transfer over into perceptions of safety.

\begin{tabular}{|c|c|c|}
\hline \multirow[b]{2}{*}{ Item } & \multicolumn{2}{|c|}{ Scores } \\
\hline & Index & Mean \\
\hline Clean Buses and Stop & 90.20 & 3.40 \\
\hline Safety at Bus Stop & 101.01 & 3.96 \\
\hline Safety on Bus & 99.20 & 4.11 \\
\hline Safety Getting off Bus & 101.61 & 4.12 \\
\hline Overall Mean & & 3.90 \\
\hline
\end{tabular}




\section{Customer Satisfaction Model}

The following chart indicates which of Key West's factors are the most important. The most influential factors are those with the largest importance values. For instance, since Cleanliness/Safety, Span of Service, and System Design have the highest importance values, the Key West transit agency should devote the most time and energy to these categories in order to improve customer satisfaction ratings. By looking at the individual items in each of these three constructs, management can determine which items to focus on.

\section{Customer Satisfaction Model: Key West}

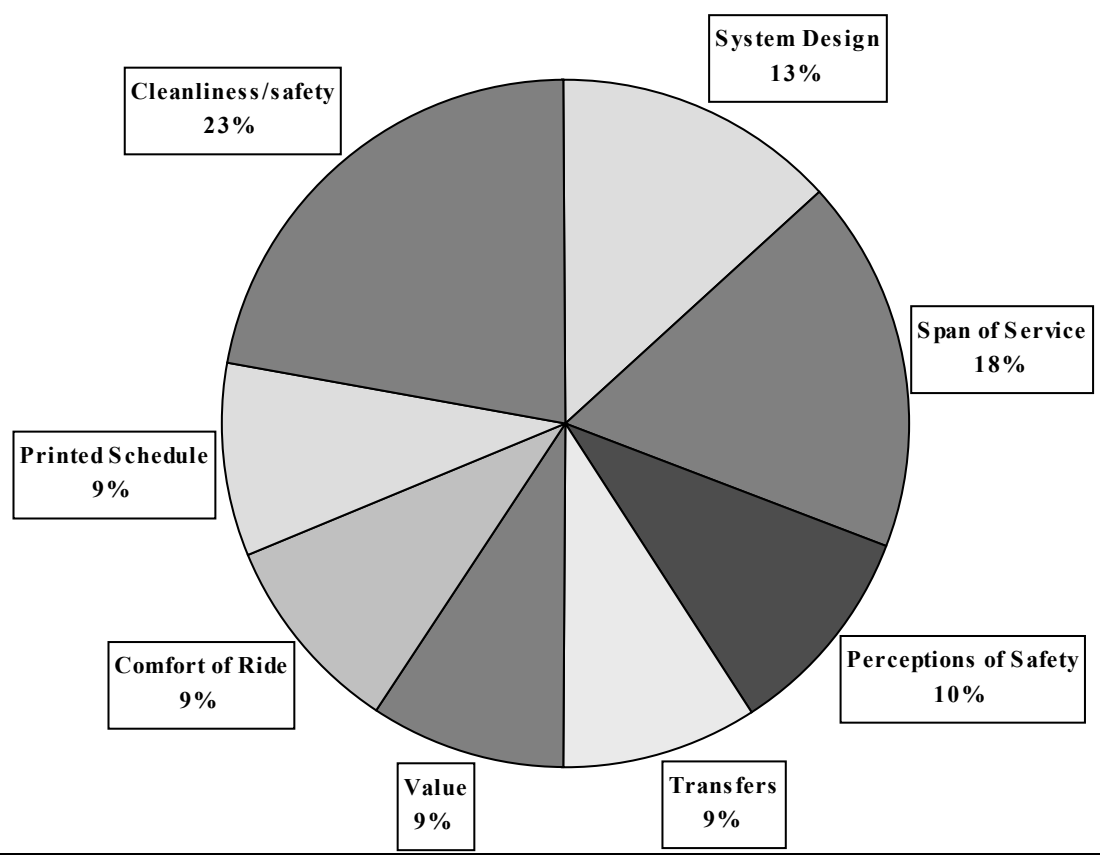

Figure 18: Key West Customer Satisfaction Model 


\section{$\underline{\text { Demographic Analysis }}$}

\section{Income}

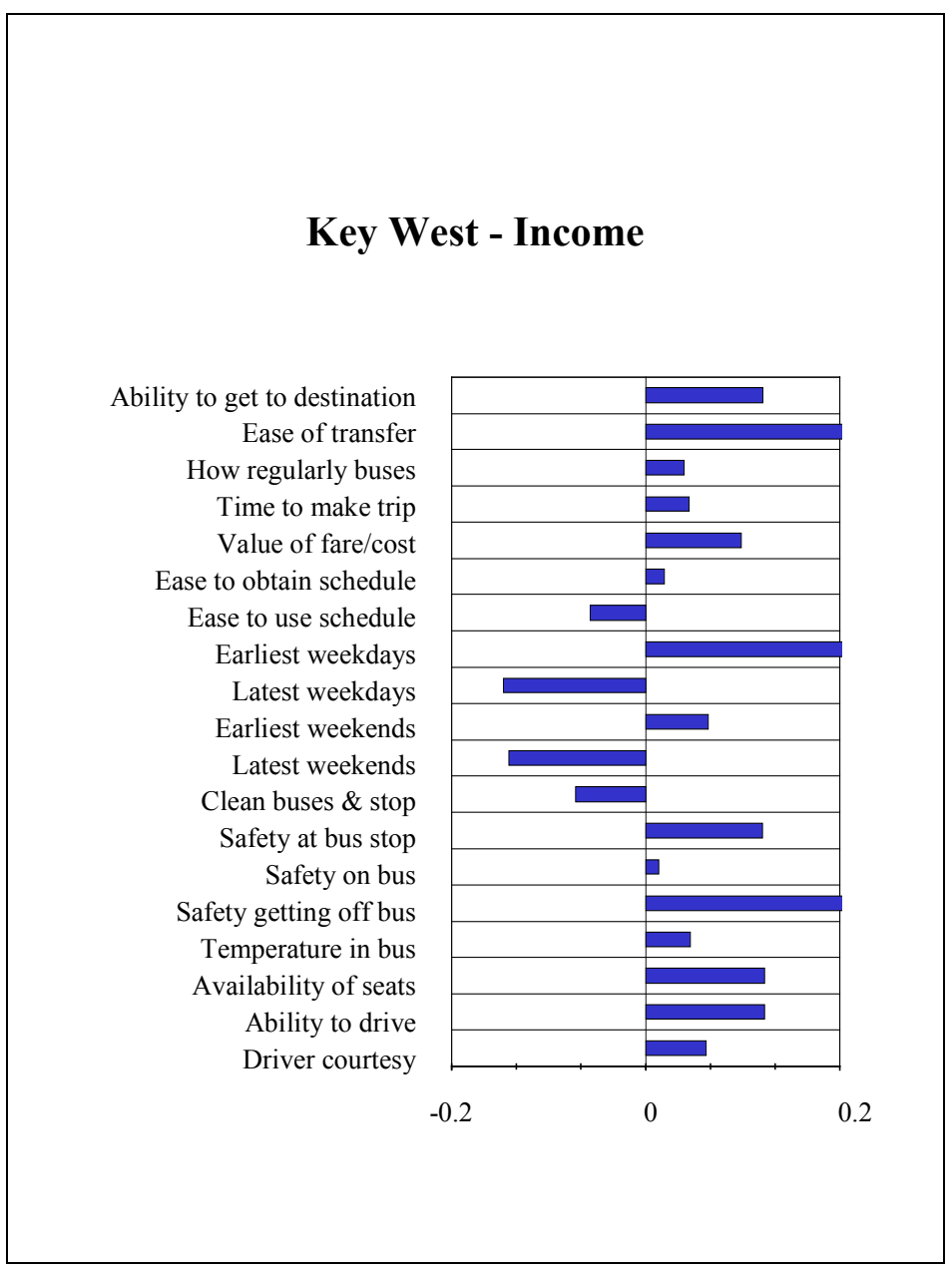

Figure 19: Key West Correlation of income with satisfaction items

Key West's transit service encompasses four island-based routes that serve tourists as well as its citizens. Theoretically, a tourist will have more disposable income than Key West's average citizen will. Therefore, the survey's high-income categories may include a majority of tourists rather than the Key West public.

For the most part, higher income riders are more satisfied with transit service than lower income riders. This finding is likely due to the fact that higher income riders are more likely to be choice riders and hence will only use the bus when it is convenient for them. Thus those of higher income who are potential riders who feel that the bus is not 
convenient (in terms of routes, span of service, etc.) will choose not to ride the bus and would therefore not be sampled. Lower income riders do not have this choice.

The items with which lower income riders are more satisfied include ease of using schedules, cleanliness of buses and stop, and ending service times. Higher frequency of riding the bus probably makes lower income riders more aware of how to use schedules. Lower income riders also probably use this familiarity to set more realistic expectations for cleanliness of the bus-riding experience than higher income riders.

High-income patrons are more satisfied with safety at the bus stop, safety on the bus, and safety getting off bus because due to their higher income status they live in lower crime areas and are less likely to travel on the bus at night. Low-income riders rely on the bus more and may live in higher crime areas and must travel on the bus regardless of the time of day. They are, therefore, likely to be less satisfied with their safety on the bus.

Higher income individuals perceived comfort factors to be more satisfactory. They rated temperature in bus, seat availability, ability to drive, and driver courtesy higher than lowincome riders. Again, this probably relates to the fact that higher income individuals tend to ride buses in the nicer neighborhoods. Less passenger crowding causes temperature in bus and seat availability to be rated higher. Roads that are located within high-income neighborhoods tend to be maintained better. Therefore, the 'ability to drive' rating may be higher due to better maintenance (i.e. fewer potholes) of the roads used on these routes. Moreover, drivers may be more relaxed and friendly when encountering a wellmaintained stretch of road. 


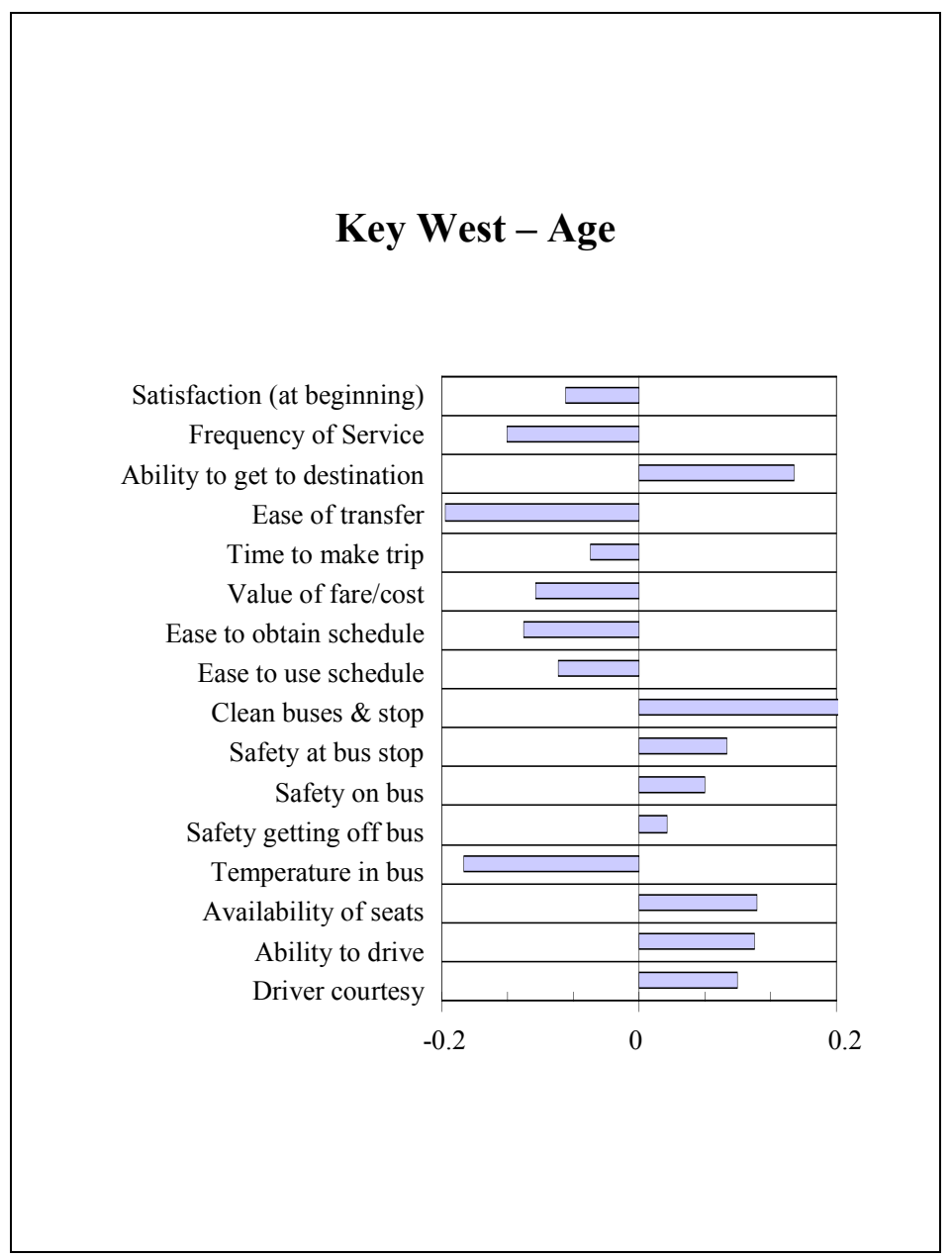

Figure 20: Key West Correlation of age with satisfaction items

Unlike the Florida transit systems' overall satisfaction tendencies relating to age, only half of the satisfaction factors are positively correlated with age. Different experiences between the older and younger individuals may cause the satisfaction differences between younger and older individuals. Individual time constraints and amount of leisure time can influence a passenger's satisfaction level.

A combination of familiarity with surroundings as well as non-peak trips can lead to higher satisfaction ratings. Elderly people tend to utilize transit services in their particular neighborhood. Therefore, they are usually more familiar with their surroundings. Those who are more familiar with their surroundings tend to be generally more comfortable. Familiarity can lead older people to bestow higher ratings to driver courtesy, ability to drive, and safety factors. Also, since older riders tend to use the bus 
for short trips within their neighborhood, they may be more satisfied with transit's ability to get to their preferred destination. Riding a bus during non-peak trips increases the tendency of passengers to be more satisfied with the number of seats available on bus and cleanliness/comfort of bus. For example, a bus is perceived to be cleaner when there is less passenger congestion. Therefore, since older clients tend to ride the buses during non-peak service times, they tend to perceive the bus as being more clean/comfortable than younger passengers.

Frequent bus usage can procure higher satisfaction ratings. For example, those who use the bus for longer trips will usually rate ease of transfer, ease to obtain schedule, and ease to use schedule higher. Since younger people tend to ride the bus more frequently, they often rate these factors better than older adults.

Some of the correlations do not have explanations. The small sample size can give unreliable results. The negative correlations between age and value of fare/trip, time to make trip, and frequency of service are difficult to explain. 


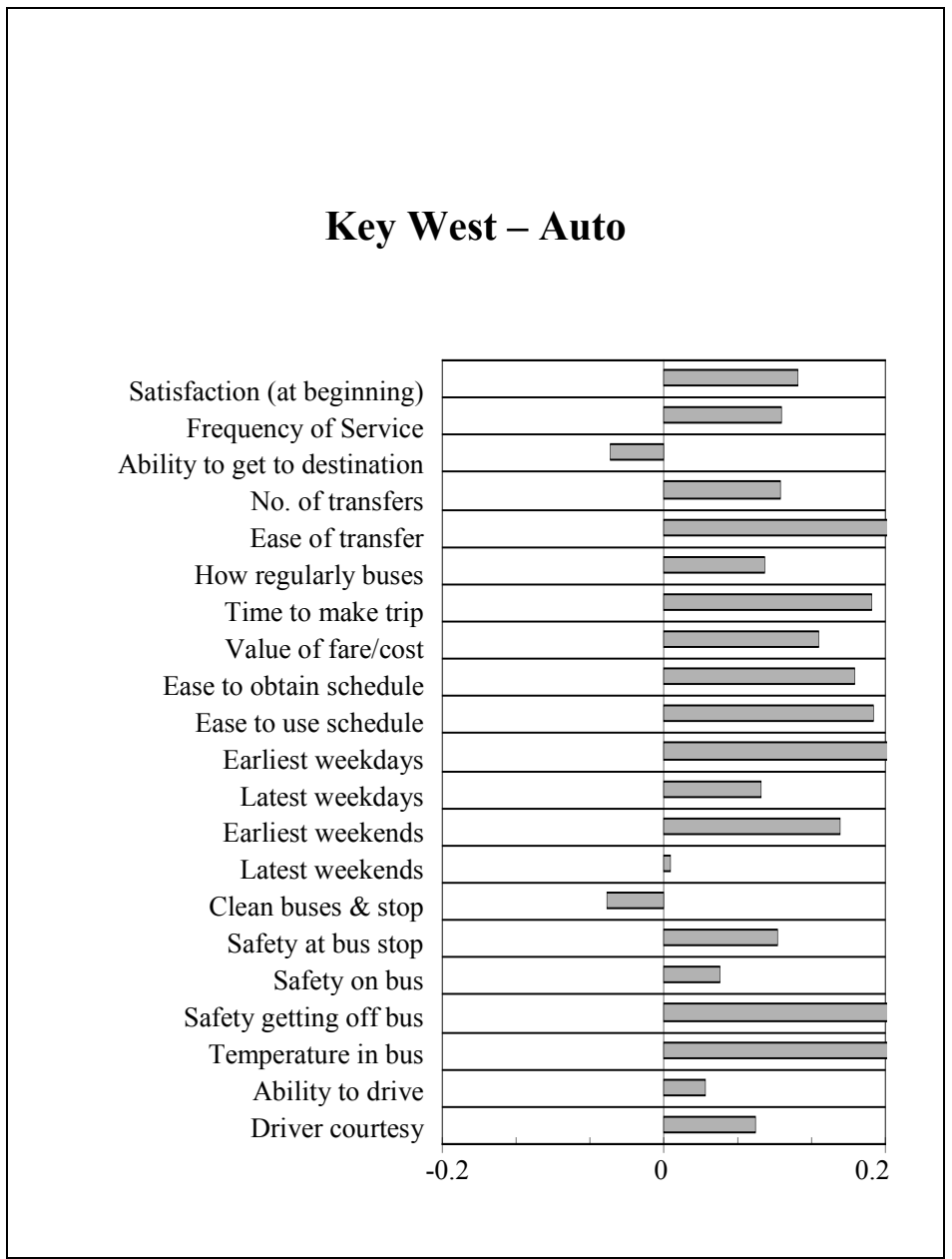

Figure 21: Key West Correlation of auto ownership with satisfaction items

Auto ownership impacts these satisfaction levels because there is a difference between riding buses due to convenience versus riding them out of necessity. Key West riders who own cars are much more satisfied with transit. A strong, positive correlation exists between number of automobiles and satisfaction.

Not surprisingly, automobile owners tended to be more satisfied with time service begins and ends for weekdays and weekends. Households that own automobiles have the luxury of 24-hour transportation. In contrast, households without vehicles are constrained by transit service times, taxi costs, and/or friends' schedules. Therefore, persons without automobiles are less satisfied with transit service begin and end times. 
As car ownership increases, satisfaction for number of transfers increases. This positive relationship reveals a car owner's tendency to utilize transit for short trips. In contrast, those without cars rely on transit for all destinations. Usually, longer trips require more transfers, which can be seen as cumbersome by those who use transit as their only reliable form of transportation.

Unlike the All Systems results, satisfaction of the value of fare/cost is positively correlated with number of automobiles in household. Value of fare/cost was perceived to be more satisfying by those with vehicles. Assuming passengers who own cars receive a larger income, the fare would be a smaller percentage of take home salary for them. Also, those with vehicles know the true cost of providing transportation and would thereby consider transit service a better buy than those without a vehicle.

Finally, lower income passengers perceived bus cleanliness and comfort to be more satisfactory than higher income passengers. This suggests that the riders who own automobiles are used to choosing their passengers. Auto owners may not be as comfortable with their fellow transit clientele and may be especially uncomfortable on crowded buses. 


\section{$\underline{\text { Recommendations }}$}

From the customer satisfaction model, it is possible to construct an "importanceperformance" matrix that graphically illustrates current bus riders' perceptions of CityTran's operations.

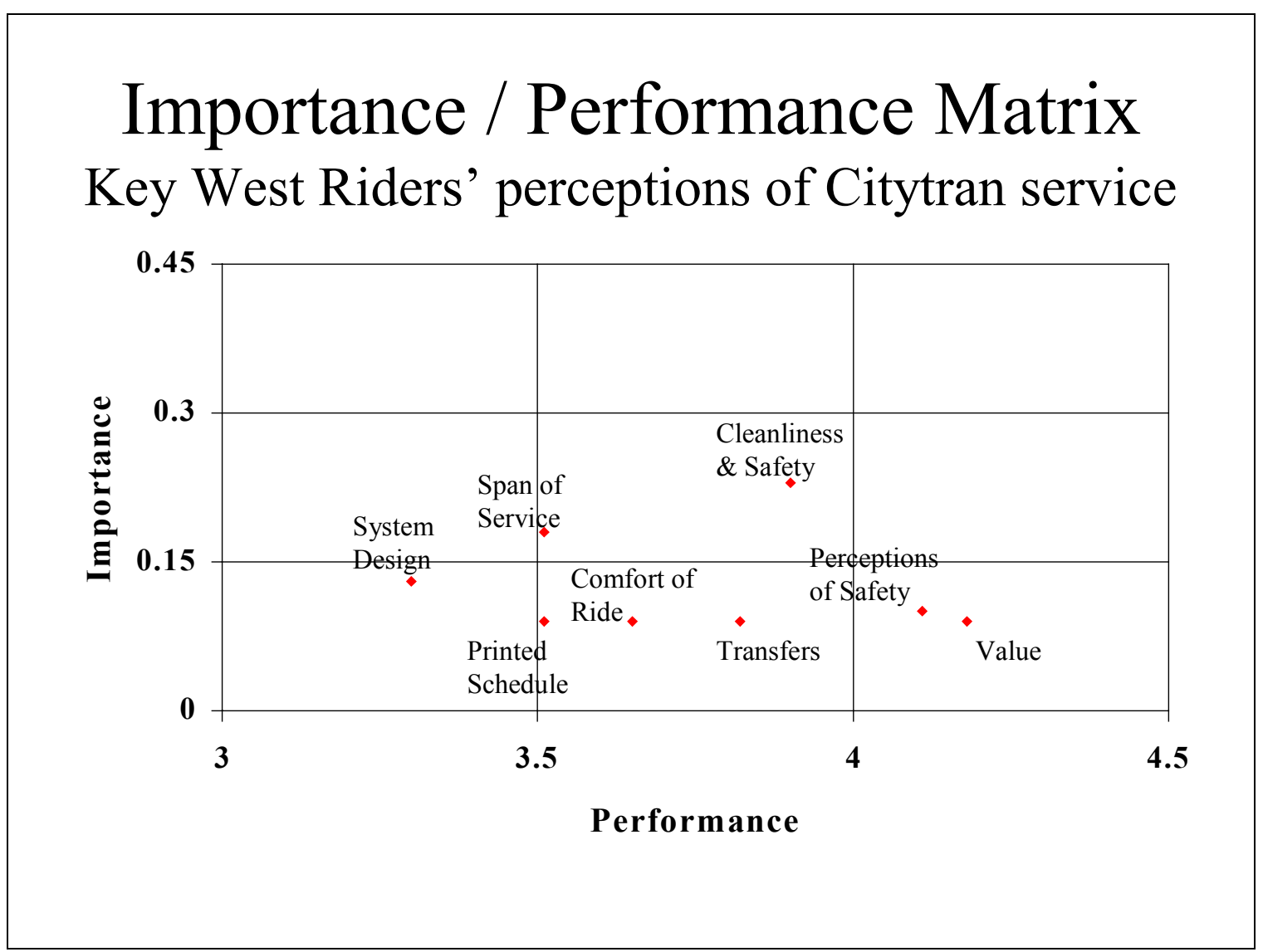

Figure 22: Key West Importance / Performance Matrix 
The chart has been divided into nine regions, reflecting various combinations of low, medium, and high performance and low, medium, and high importance. Borderline figures are interpreted as being in the higher of the importance categories they border on, but the lower of the performance categories. This provides the most conservative interpretation of the results. The interpretations of the chart regions are done as follows:

\begin{tabular}{|c|c|c|c|}
\hline \multicolumn{4}{|c|}{$\begin{array}{c}\text { Table } 6 \\
\text { Interpretations of CityTran's Chart Regions }\end{array}$} \\
\hline \multicolumn{2}{|c|}{ Chart region } & \multirow[b]{2}{*}{ Interpretation } & \multirow[b]{2}{*}{ Areas } \\
\hline Importance & Performance & & \\
\hline Low & High & Possibly reduce focus on this area & $\begin{array}{l}\text { Value, Perceptions of } \\
\text { safety }\end{array}$ \\
\hline Low & Medium & Maintain performance - no action & $\begin{array}{l}\text { Transfers, Comfort of } \\
\text { Ride, Printed Schedule }\end{array}$ \\
\hline Low & Low & Maintain performance - no action & System Design \\
\hline Medium & High & Maintain performance - no action & \\
\hline Medium & Medium & Maintain performance - no action & $\begin{array}{l}\text { Cleanliness \& safety, } \\
\text { Span of Service }\end{array}$ \\
\hline Medium & Low & Investigate for improvements & \\
\hline High & High & $\begin{array}{l}\text { Maintain performance - vigorous } \\
\text { quality checks, constant attention }\end{array}$ & \\
\hline High & Medium & Investigate for improvements & \\
\hline High & Low & Critical improvement area & \\
\hline
\end{tabular}


In CityTran's case, the Value and Perceptions of Safety factors fall into the "possibly reduce focus" area, while the Transfers, Comfort of Ride, Printed Schedule, System Design, Safety \& Cleanliness and Span of Service factors are all in the "maintain performance - no action" areas. Examination of the chart shows that Span of Service and System Design are the items that probably most warrant potential corrective action.

The individual Span of Service items that CityTran scores particularly low on are:

\begin{tabular}{|lc|c|}
\hline \multirow{2}{*}{\multicolumn{1}{|c|}{ Item }} & \multicolumn{2}{c|}{ Scores } \\
\cline { 2 - 3 } \multicolumn{1}{|c|}{} & Index & Mean \\
\hline Latest weekdays & 106.78 & 3.32 \\
\hline Latest weekends & 103.03 & 3.20 \\
\hline
\end{tabular}

The System Design items with low scores are:

\begin{tabular}{|lc|c|}
\hline \multirow{2}{*}{\multicolumn{1}{c}{ Item }} & \multicolumn{2}{c|}{ Scores } \\
\cline { 2 - 3 } & Index & Mean \\
\hline Frequency of Service & 90.19 & 3.12 \\
\hline Time to Make Trip & 92.67 & 3.45 \\
\hline How regularly Buses Arrive On Time & 82.18 & 2.72 \\
\hline
\end{tabular}

Key West's customers are usually more satisfied with Span of Service than the other transit agencies' customers. Key West's customers are primarily satisfied with service begin times. However, they are slightly dissatisfied with service end times for both weekdays and weekends. Therefore, Key West may want to focus on trying to increase its evening service hours. The latest weekend service has the lowest mean. This indicates that it would be wise to increase weekend evening hours first.

"Frequency of service," "time to make trip," and "how regularly buses arrive on time" are three items that could use improvement. In contrast, passengers were happy with the "ability to get where" item located within System Design. It is recommended that an 
interim network should be established per the August 2000 Comprehensive Operations Analysis. With six buses, the suggested network would be able to operate with at least 30-minute frequencies while providing an incremental increase in the annual revenue hours of service. Moreover, operating an adjusted schedule would allow the transit system to add buses to routes in times when traffic volumes and/or ridership are heavy, thereby allowing regularity of buses to not be compromised.

According to the individual index and mean scores shown in Table 11-VIII, Key West is doing a good job with respect to the Cleanliness/Safety category. For instance, the items "safety at bus stop" and "safety getting off bus" are better than average. Also, the overall mean indicates that this category is one of Key West's strengths. Therefore, Key West should continue providing its exemplary service in safety and cleanliness.

Small sample sizes did not permit a thorough demographic analysis of other demographic categories (such as race and gender) for Key West's operations. 


\section{$\underline{\text { JTA }}$}

$\underline{\text { Factor Analysis }}$

Factor 2 - $\quad$ Span of Service

Major loadings - $\quad$ SQ17 Latest weekends

SQ16 Earliest weekends

SQ15 Latest weekdays

SQ14 Earliest weekdays

Minor loading - $\quad$ SQ5 Frequency of service

This construct relates to customer satisfaction with span of transit service availability.

The components are satisfaction with earliest weekday, latest weekday, earliest weekend day, and latest weekend day service. Frequency of service also slightly loaded on this factor.

\begin{tabular}{|lc|c|}
\hline \multicolumn{2}{|c|}{ Table 7-I: JTA Factor 2 - Span of Service } \\
\cline { 2 - 3 } & \multicolumn{2}{c|}{ Scores } \\
\cline { 2 - 3 } \multicolumn{1}{|c|}{ Item } & Index & Mean \\
\hline Latest Weekends & 86.50 & 2.68 \\
\hline Earliest Weekends & 85.20 & 2.94 \\
\hline Latest weekdays & 91.63 & 2.85 \\
\hline Earliest Weekdays & 90.69 & 3.40 \\
\hline Frequency of Service & 84.52 & 2.93 \\
\hline Overall Mean & & $\mathbf{2 . 9 6}$ \\
\hline
\end{tabular}


Factor 3 - Perceptions of Safety

Major loadings - $\quad$ SQ22 Safety at bus stop

SQ24 Safety getting off bus

SQ20 Clean buses and stop

SQ23 Safety on bus

Minor loading - $\quad$ SQ26 Temperature in bus

SQ27 Availability of seats

This construct relates to customer satisfaction with safety at bus stop, safety getting off bus, clean buses and stops, safety on bus, as well as temperature on bus. The variable availability of seats also slightly loaded on this factor. The temperature/availability of seats part of the construct may reflect bus crowding as a safety issue - the more crowded the bus, the less safe the patrons feel. The connection between safety and cleanliness requires some explanation. One possibility is that the passenger construct relates to the apparent level of concern and respect the transit agency has for their passengers.

Cleanliness is a very common way of showing respect and concern for customers - in restaurants, retail businesses, even in personal interactions. One of the correlates of transit agency concern for customers could be safety at bus stops, since the perception that the agency is taking care of its equipment and facilities may transfer over into perceptions of safety.

\begin{tabular}{|c|c|c|}
\hline \multirow[t]{3}{*}{ Table 7-II: J } & ptions & \\
\hline & \multicolumn{2}{|c|}{ Scores } \\
\hline & Index & Mean \\
\hline Safety at Bus Stop & 88.18 & 3.45 \\
\hline Safety Getting off Bus & 91.32 & 3.71 \\
\hline Clean Buses and Stop & 92.86 & 3.50 \\
\hline Safety on Bus & 94.18 & 3.90 \\
\hline Temperature in Bus & 92.06 & 3.54 \\
\hline Availability of Seats & 98.06 & 3.82 \\
\hline Overall Mean & & 3.65 \\
\hline
\end{tabular}


Factor $4-\underline{\text { Transfers }}$

Major loadings - $\quad$ SQ7 Number of transfers

SQ8 Ease of transfer

SQ6 Ability to where you want to go

Minor loading - $\quad$ SQ5 Frequency of service

This construct relates to customer satisfaction with the number of transfers, ease of transfers and ability to get where you want to. Frequency of service also slightly loads onto this factor. Clearly the number and ease of making transfers has a major impact on customer perception of being able to get where they want to go, and frequency of service impacts the ease of making transfers.

\begin{tabular}{|lc|c|}
\hline \multicolumn{3}{|c|}{ Table 7-III: JTA Factor 4 - Transfers } \\
\cline { 2 - 3 } & \multicolumn{2}{c|}{ Scores } \\
\cline { 2 - 3 } Item & Index & Mean \\
\hline Number of Transfers & 87.84 & 3.18 \\
\hline Ease of Transfers & 82.34 & 3.21 \\
\hline Ability to get where you want to go & 91.46 & 3.58 \\
\hline Frequency of Service & 84.52 & 2.93 \\
\hline Overall Mean & & 3.22 \\
\hline
\end{tabular}


Factor 7 - $\quad \underline{\text { Schedule }}$

Major loadings - $\quad$ SQ13 Ease to use schedule

SQ12 Ease to obtain schedule

Minor loading - $\quad$ SQ14 Earliest weekdays

The construct relates to customer experience using schedules. The main variable in this construct is satisfaction with the ease of using the schedule. Predictably, other variables loading on this factor are ease to obtain schedule and how regularly the buses arrive on time.

\begin{tabular}{|lc|c|}
\hline \multicolumn{3}{|c|}{ Table 7-IV: JTA Factor 7 - Schedule } \\
\cline { 2 - 3 } & \multicolumn{2}{c|}{ Scores } \\
\cline { 2 - 3 } Item & Index & Mean \\
\hline Ease to Use Schedule & 91.40 & 3.76 \\
\hline Ease to Obtain Schedule & 90.14 & 3.69 \\
\hline Earliest Weekdays & 90.69 & 3.40 \\
\hline Overall Mean & 3.62 \\
\hline
\end{tabular}


Factor 8 - $\quad$ Value/Timeliness of Service

Major loadings - $\quad$ SQ11 Value of fare/cost

SQ9 How regularly buses arrive on time

SQ10 Time to make trip

SQ5 Frequency of service

Minor loading - $\quad$ SQ26 Temperature in bus

In this construct the main elements are customer satisfaction with value of fare/cost and timeliness of service, which includes time to make trip and how regularly buses arrive on time. This indicates that Jacksonville customers strongly correlate their perceptions of value with operational characteristics of system performance. The variable frequency of service is also slightly loaded onto this factor.

\begin{tabular}{|lc|c|}
\hline \multicolumn{2}{|c|}{ Table 7-V: JTA Factor 8 - Value/Timeliness of Service } \\
\hline \multicolumn{2}{|c|}{ Item } & Scores \\
\cline { 2 - 3 } & Index & Mean \\
\hline Value of Fare/Cost & 86.03 & 3.53 \\
\hline How Regularly Buses Arrive On Time & 92.98 & 3.07 \\
\hline Time to Make Trip & 85.08 & 3.17 \\
\hline Frequency of Service & 84.52 & 2.93 \\
\hline Temperature in Bus & 92.06 & 3.54 \\
\hline Overall Mean & & 3.25 \\
\hline
\end{tabular}


Factor 9 - $\quad$ Experience of the Bus Ride

Major loadings - $\quad$ SQ29 Driver courtesy

SQ28 Ability to drive

SQ27 Availability of seats

Minor loadings - $\quad$ SQ23 Safety on bus

\section{SQ26 Temperature on bus}

This construct includes customer satisfaction with driver courtesy, ability to drive, and availability of seats. The variables, safety on bus and temperature on bus also slightly load on this factor. The construct encompasses all the major experiential elements involved in the bus ride - meeting the driver, finding a seat, the ride itself, the heat in the bus, and whatever perceptions of safety one might have while riding.

\begin{tabular}{|lc|c|}
\hline \multicolumn{2}{|c|}{ Table 7-VI: JTA Factor 9 - Experience of the Bus Ride } \\
\hline \multirow{2}{*}{ Item } & Index & Mean \\
\cline { 2 - 3 } & 93.65 & 4.04 \\
\hline Driver Courtesy & 97.83 & 4.19 \\
\hline Ability to Drive & 98.06 & 3.82 \\
\hline Availability of Seats & 94.18 & 3.90 \\
\hline Safety on Bus & 92.06 & 3.54 \\
\hline Temperature on Bus & 3.87 \\
\hline Overall Mean & & \\
\hline
\end{tabular}




\section{$\underline{\text { Customer Satisfaction Model }}$}

The following chart indicates which of Jacksonville's factors are the most important. The most influential factors are those with the largest importance values. For instance, since Value/Timeliness, Transfers, and Span of Service have the highest importance values, JTA should devote the most time and energy to these categories in order to improve customer satisfaction ratings. By looking at the individual items in each of these three constructs, management can determine which items to focus on.

\section{Customer Satisfaction Model: JTA}

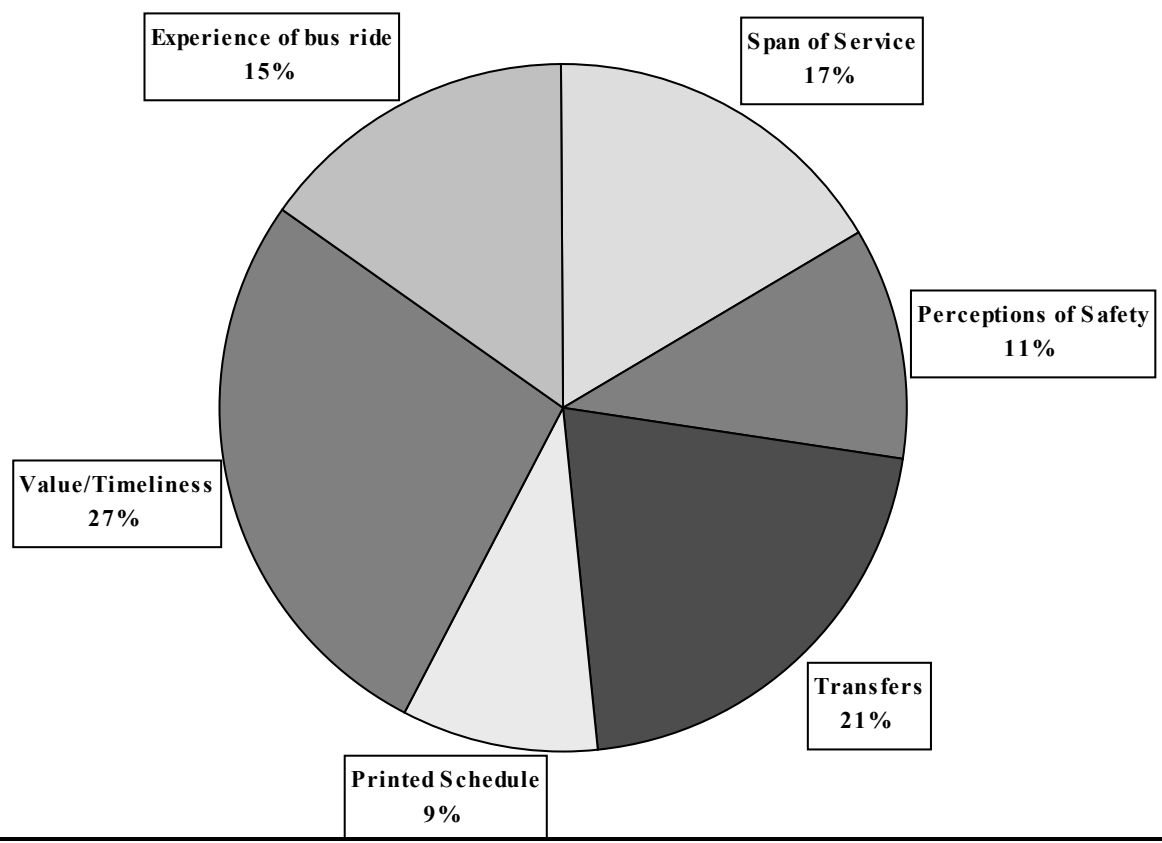

Figure 23: JTA Customer Satisfaction Model 
Demographic Analysis

\section{Income}

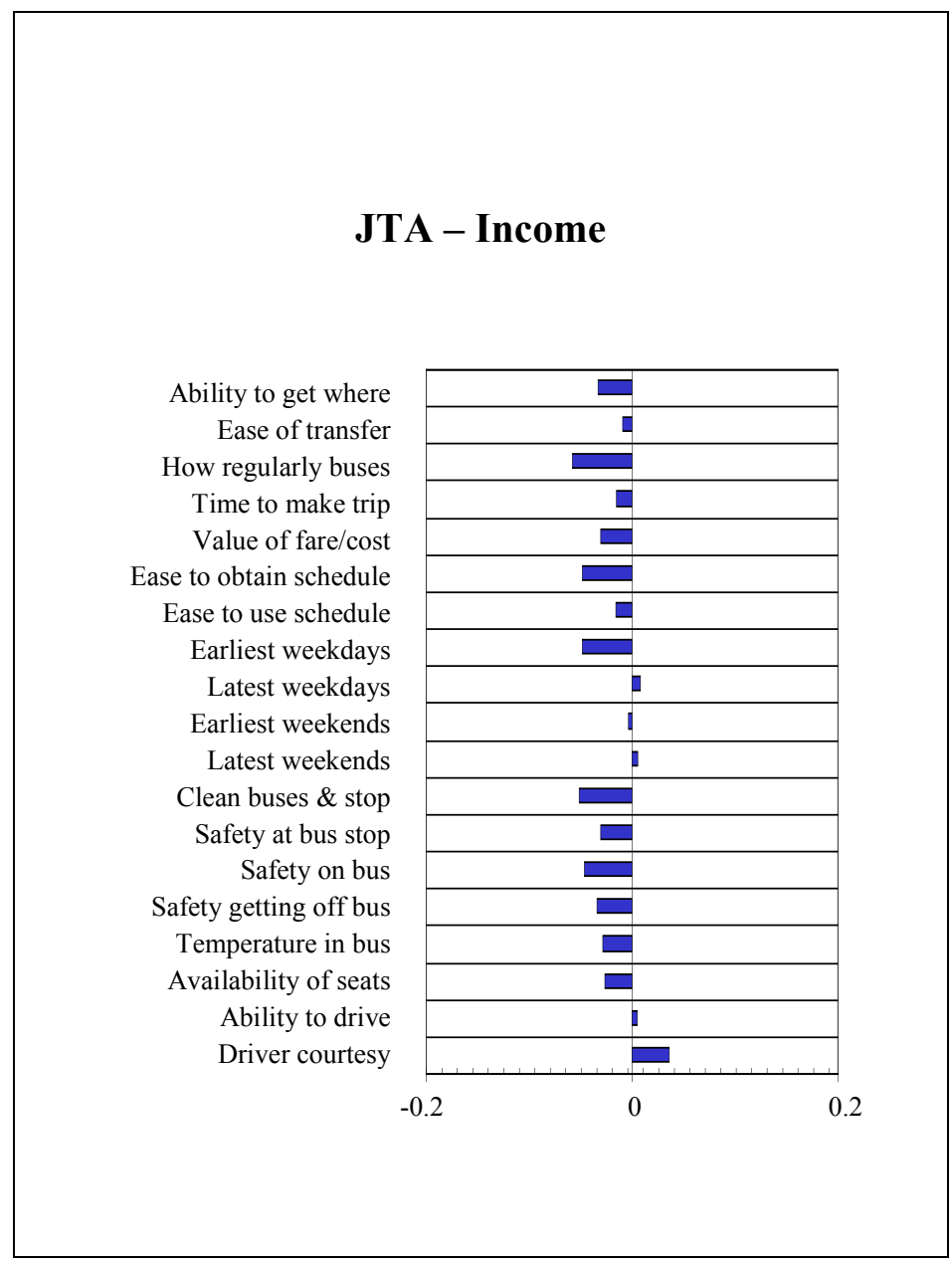

Figure 24: JTA Correlation of income with satisfaction items

Analysis of a non-linearly related item, satisfaction with frequency of service, showed that those with incomes of $\$ 45,000-\$ 55,000$ were significantly less satisfied than all other groups with frequency of service.

Unlike the Income Total correlation, for the most part, JTA's lower income riders are more satisfied with transit service than higher income riders. In fact, its lower income riders are more satisfied with all but one of the significant categories. Driver Courtesy was the only significant category that was positively correlated with income: as income increases, satisfaction with driver courtesy increases. As income decreases satisfaction of 
transit service, value/cost, vehicle conditions, and personal safety increase. According to the survey, lower income individuals are more satisfied with transit's bus stop locations, bus regularity, ease of transfer, and time to make trip. Since lower income individuals utilize transit frequently, they are able to make better time-frame judgments.

Higher frequency of riding the bus makes lower income riders familiar with transit conditions. Lower income riders probably use this familiarity to set more realistic expectations for these elements of the bus-riding than higher income riders. Thus the experiences that occur for which no prior expectation can reasonably be formed without prior use of the bus (such as on-time arrivals, cleanliness and comfort, temperature on the bus, availability of seats, and issues relating to the driver) may be expected to get higher ratings among lower-income users. Moreover, they are usually more aware of where to obtain schedules, how to use schedules, and how to transfer.

Unlike the overall income analysis of all Florida systems, Jacksonville's fare structure is perceived to be more valuable as income decreases. This may be due to the particular fare structure used by Jacksonville. It might be worthwhile to compare the fare structures among Florida transit systems in order to get a better understanding of why this correlation is different from the statewide statistics. 


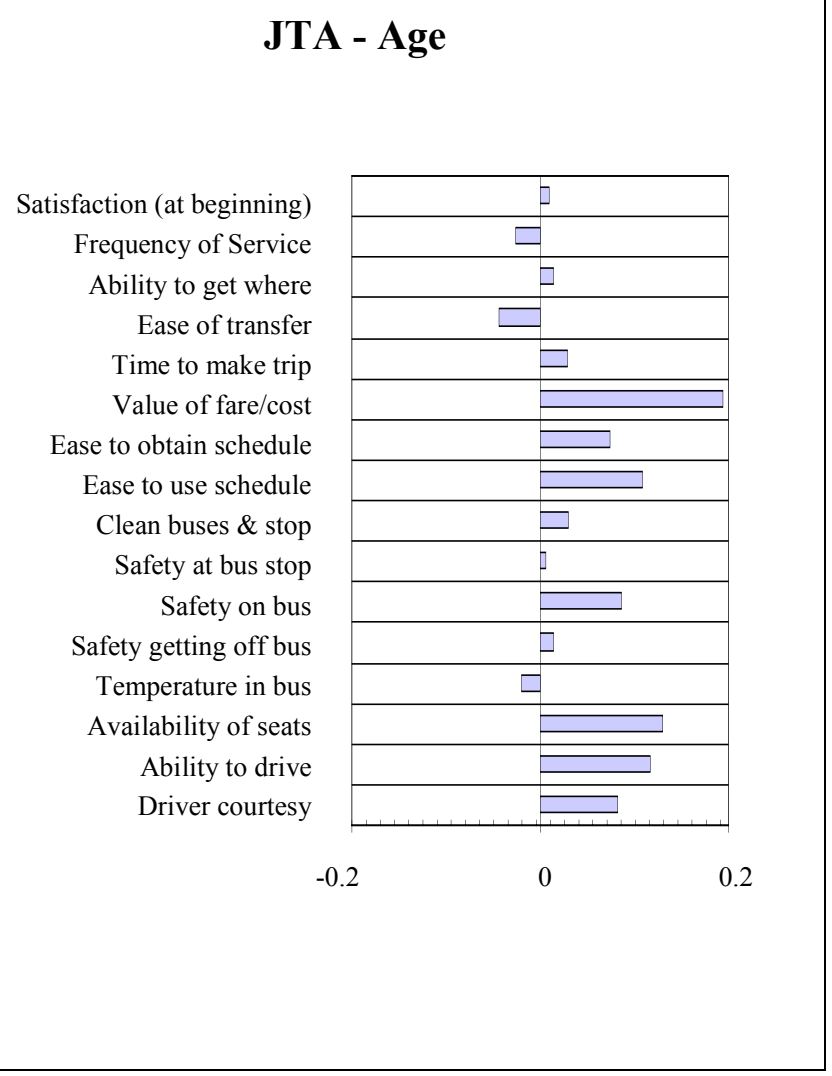

Figure 25: JTA Correlation of age with satisfaction items

An analysis of a non-linearly related item, number of transfers, showed that riders under 17 had the highest level of satisfaction with number of transfers. Generally, those under 17 and over 60 had the highest level of satisfaction with earliest/latest service times.

Almost all of the satisfaction factors are positively correlated with age. Overall, older respondents are more satisfied with transit service. As age increases, satisfaction increases for all but three categories: frequency of service, ease of transfer, and temperature in bus. Compared to the All Systems findings, all but two categories frequency of service and ease of transfer, suggest similar correlations. JTA differs because its younger passengers appear to be more satisfied with the frequency of service and ease of transfer. 
Fare structure causes passengers to rate the value of transit differently. JTA's strongest positive correlation is between fare value and age. Since Jacksonville's fare is free for senior citizens over age 60, it is not surprising that the value of trip satisfaction increases with age. Moreover, JTA's senior citizens may want more frequent service and smoother transfers to take advantage of their free fare status.

Different experiences between the older and younger individuals may cause the satisfaction differences between younger and older individuals. Individual time constraints and amount of leisure time can influence a passenger's satisfaction level. For instance, younger people are usually on a tighter schedule, such as arriving to work on time. Therefore, younger people will usually be more conscience of time delays. This can lead younger people to be less satisfied with convenience of routes and dependability. Also, younger passengers usually have less leisure time. Assuming that they would like to gain additional leisure time, their dissatisfaction with time to make trip is understandable. Moreover, younger riders tend to rate days of service with less satisfaction. Assuming that younger persons have less errand time during the weekday, they may perceive days of service to be dissatisfactory due to the fact that they need to take more trips on the weekend to fulfill all of their errands.

A combination of familiarity with surroundings as well as non-peak trips can lead to higher satisfaction ratings. Elderly people tend to utilize transit services in their particular neighborhood. Therefore, they are usually more familiar with their surroundings. Those who are more familiar with their surroundings tend to be generally more comfortable. Familiarity can lead older people to bestow higher ratings to driver courtesy, ability to drive, ease to obtain schedule, ease to use schedule, and safety factors. Riding a bus during non-peak trips increases the tendency of passengers to be more satisfied with the number of seats available on bus and cleanliness/comfort of bus. For example, a bus is perceived to be cleaner when there is less passenger congestion. Therefore, since older clients tend to ride the buses during non-peak service times, they tend to perceive the bus as being more clean/comfortable than younger passengers. 


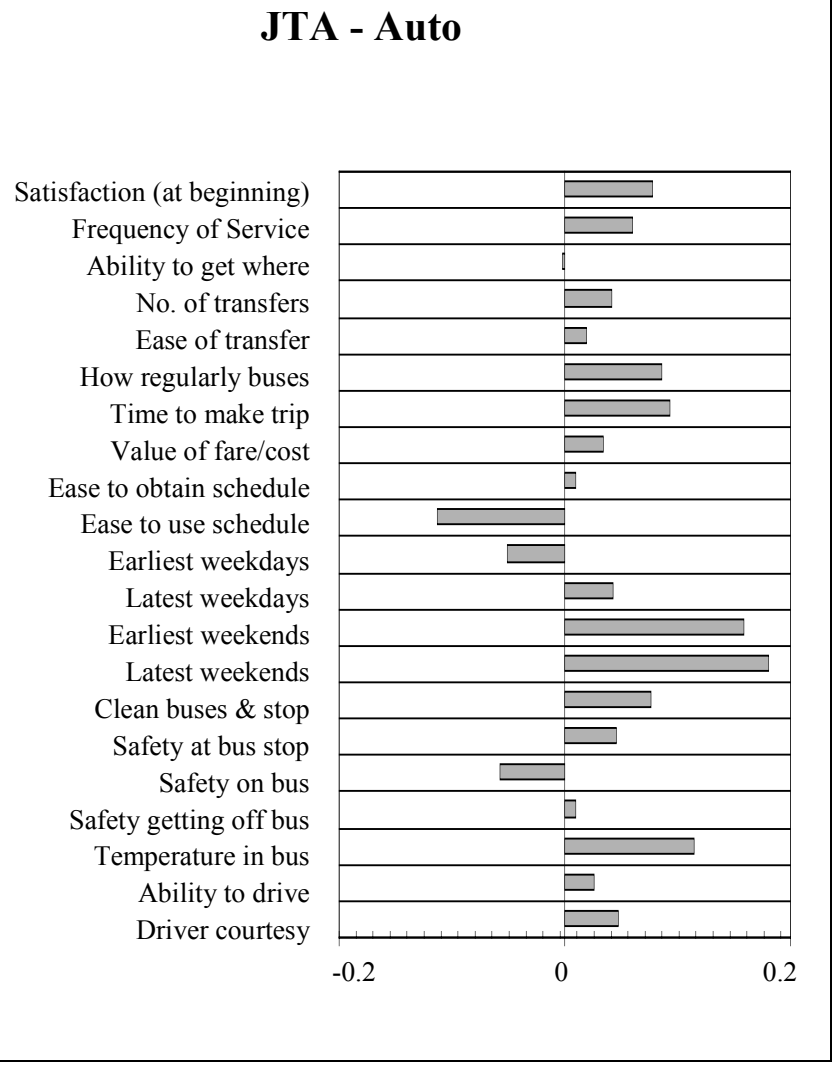

Figure 26: JTA Correlation of auto ownership with satisfaction items

An analysis of a non-linearly related item, availability of seats, showed that those with 2 vehicles were significantly more satisfied than those with 1 vehicle in their household.

Due to the fact that those who own automobiles have an alternate form of transportation at their disposal, it is not surprising to obtain many positive satisfaction correlations between auto ownership and transit service categories. In fact, the positive satisfaction correlations between auto ownership and service frequency, number of transfers, ease of transfer, time to make trip, bus regularity, and weekend service schedule suggest that auto owners ride the bus when it is a convenience. Moreover, choice riders may choose routes that are more direct (i.e. express, limited stop). 
The JTA survey related positive correlation between number of automobiles and satisfaction with time service begins and ends for weekdays and weekends. Households that own automobiles have the luxury of 24-hour transportation. In contrast, households without vehicles are constrained by transit service times, taxi costs, and/or friends' schedules. Therefore, persons without automobiles are less satisfied with transit service begin and end times.

As car ownership increases, satisfaction for number of transfers increases. This positive relationship reveals a car owner's tendency to utilize transit for short trips. In contrast, those without cars rely on transit for all destinations. Usually, longer trips require more transfers, which can be seen as cumbersome by those who use transit as their only reliable form of transportation.

Those who depend on transit as their only source of reliable transportation utilize transit even during inconvenient times in order to get their weekly tasks and errands accomplished. Also, transit dependants are more apt to use service that has many stops in order to complete all of their work, grocery store, daycare, and mall trips. According to the survey JTA's schedule is perceived to be easier to use by those passengers who do not have automobiles. As previously stated, familiarity with bus schedules and bus routes will lead persons without cars to be more satisfied with ease to use schedule.

Unlike the All Systems results, satisfaction of the value of fare/cost is positively correlated with number of automobiles in household. Value of fare/cost was perceived to be more satisfying by those with vehicles. Assuming passengers who own cars receive a larger income, the fare would be a smaller percentage of take home salary for them. Also, those with vehicles know the true cost of providing transportation and would thereby consider transit service a better buy than those without a vehicle.

Those who are choice riders may experience transit differently than non-choice riders. Choice riders would only use transit if they felt safe, comfortable, and respected. Therefore, positive correlations between auto ownership and factors such as temperature in bus, clean buses and stop areas, driver courtesy, ability to drive, safety at bus stop, and safety getting off bus are understandable. However, a negative correlation between auto ownership and safety on bus suggests that those with automobiles feel safer in their cars than surrounded by strangers. 


\section{Ethnicity}

\begin{tabular}{|c|c|c|c|}
\hline & Table 8-I: JTA Ethnicity & White & Black \\
\hline Sample size & & $\mathbf{9 9 3}$ & $\mathbf{2 1 4 7}$ \\
\hline & & & \\
\hline SQ13 & Ease to use schedule & 3.66 & 3.85 \\
\hline SQ29 & Driver courtesy & 4.17 & 3.98 \\
\hline SQ11 & Value of fare/cost & 3.69 & 3.46 \\
\hline SQ26 & Temperature in bus & 3.71 & 3.45 \\
\hline
\end{tabular}

Blacks had higher levels of satisfaction with ease of using schedule, probably due to more frequent use of the bus system and hence greater familiarity. Lower scores on driver courtesy and temperature may be due to higher levels of ridership on routes used by blacks. Lower scores on value may be related to this situation as well, and may also be related to JTA's policy of providing free fares to seniors, which may be seen as unfair by some riders.

\begin{tabular}{|l|l|c|c|}
\hline & \multicolumn{1}{|c|}{ Table 8-II: JTA Ethnicity } & White & Hispanic \\
\hline Sample size & & $\mathbf{9 9 3}$ & $\mathbf{1 1 3}$ \\
\hline & & & \\
\hline SQ24 & Safety getting off bus & 3.75 & 4.26 \\
\hline SQ22 & Safety at bus stop & 3.53 & 3.95 \\
\hline SQ33 & Dependability of buses (on time) (combined SQ 9 \& 19) & 3.06 & 3.43 \\
\hline SQ13 & Ease to use schedule & 3.66 & 4.00 \\
\hline SQ34 & Cleanliness/comfort (combined SQ 20 \& 21) & 3.61 & 3.93 \\
\hline SQ27 & Availability of seats & 3.91 & 4.22 \\
\hline SQ17 & Latest weekends & 2.73 & 3.01 \\
\hline SQ23 & Safety on bus & 3.95 & 4.22 \\
\hline SQ08 & Ease of transfer & 3.14 & 3.38 \\
\hline SQ05 & Frequency of Service & 2.87 & 3.10 \\
\hline SQ32 & Convenience of routes (combined SQ 6 \& 18) & 3.49 & 3.70 \\
\hline SQ26 & Temperature in bus & 3.71 & 3.92 \\
\hline
\end{tabular}




\begin{tabular}{|l|l|c|c|}
\hline \multicolumn{1}{|c|}{ Table 8-III: JTA Ethnicity } & Black & Hispanic \\
\hline Sample size & & $\mathbf{2 1 4 7}$ & $\mathbf{1 1 3}$ \\
\hline & & & \\
\hline SQ24 & Safety getting off bus & 3.71 & 4.26 \\
\hline SQ22 & Safety at bus stop & 3.43 & 3.95 \\
\hline SQ34 & Cleanliness/comfort (combined SQ 20 \& 21) & 3.45 & 3.93 \\
\hline SQ26 & Temperature in bus & 3.45 & 3.92 \\
\hline SQ27 & Availability of seats & 3.77 & 4.22 \\
\hline SQ33 & Dependability of buses (on time) (combined SQ 9 \& 19) & 3.03 & 3.43 \\
\hline SQ11 & Value of fare/cost & 3.46 & 3.85 \\
\hline SQ29 & Driver courtesy & 3.98 & 4.34 \\
\hline SQ17 & Latest weekends & 2.66 & 3.01 \\
\hline SQ23 & Safety on bus & 3.90 & 4.22 \\
\hline SQ28 & Ability to drive & 4.16 & 4.45 \\
\hline
\end{tabular}

Hispanics are more satisfied than whites or blacks with many aspects of transit service, most particularly with safety, dependability, and comfort issues. Hispanics seem to feel more at ease with the bus riding experience in Jacksonville and generally more familiar with the bus system. 


\begin{tabular}{|l|l|c|c|}
\hline & \multicolumn{1}{|c|}{ Table 8-IV: JTA Ethnicity } & White & Other \\
\hline Sample size & & $\mathbf{9 9 3}$ & $\mathbf{1 8 7}$ \\
\hline & & & 3.06 \\
\hline SQ33 & Dependability of buses (on time) (combined SQ 9 \& 19) & 3.49 & 3.29 \\
\hline SQ06 & Ability to get where & 3.49 & 3.29 \\
\hline SQ32 & Convenience of routes (combined SQ 6 \& 18) & 3.53 & 3.31 \\
\hline SQ22 & Safety at bus stop & 2.87 & 2.65 \\
\hline SQ05 & Frequency of Service & 3.14 & 2.91 \\
\hline SQ08 & Ease of transfer & 3.75 & 3.50 \\
\hline SQ24 & Safety getting off bus & 3.23 & 2.96 \\
\hline SQ10 & Time to make trip & 2.84 & 2.56 \\
\hline SQ15 & Latest weekdays & 3.69 & 3.30 \\
\hline SQ11 & Value of fare/cost & 3.49 & 3.29 \\
\hline SQ32 & Convenience of routes (combined SQ 6 \& 18) & 3.53 & 3.31 \\
\hline SQ22 & Safety at bus stop & \\
\hline
\end{tabular}

\begin{tabular}{|l|l|c|c|}
\hline \multicolumn{1}{|c|}{ Table 8-V: JTA Ethnicity } & Black & Other \\
\hline Sample size & \multicolumn{1}{|c|}{$\mathbf{1 8 7}$} \\
\hline & & & \\
\hline SQ33 & Dependability of buses (on time) (combined SQ 9 \& 19) & 3.03 & 3.28 \\
\hline SQ10 & Time to make trip & 3.16 & 2.96 \\
\hline SQ24 & Safety getting off bus & 3.71 & 3.50 \\
\hline SQ12 & Ease to obtain schedule & 3.77 & 3.50 \\
\hline SQ05 & Frequency of Service & 2.98 & 2.65 \\
\hline SQ13 & Ease to use schedule & 3.85 & 3.51 \\
\hline SQ15 & Latest weekdays & 2.92 & 2.56 \\
\hline SQ06 & Ability to get where & 3.66 & 3.29 \\
\hline SQ32 & Convenience of routes (combined SQ 6 \& 18) & 3.66 & 3.29 \\
\hline SQ08 & Ease of transfer & 3.30 & 2.91 \\
\hline
\end{tabular}




\begin{tabular}{|l|l|c|c|}
\hline & \multicolumn{1}{|c|}{ Table 8-VI: JTA Ethnicity } & Hispanic & Other \\
\hline Sample size & \multicolumn{1}{|c|}{} & $\mathbf{1 1 3}$ & $\mathbf{1 8 7}$ \\
\hline & & & \\
\hline SQ17 & Latest weekends & 3.01 & 2.76 \\
\hline SQ27 & Availability of seats & 4.22 & 3.97 \\
\hline SQ28 & Ability to drive & 4.45 & 4.19 \\
\hline SQ12 & Ease to obtain schedule & 3.77 & 3.50 \\
\hline SQ23 & Safety on bus & 4.22 & 3.92 \\
\hline SQ29 & Driver courtesy & 4.34 & 4.02 \\
\hline SQ26 & Temperature in bus & 3.92 & 3.59 \\
\hline SQ10 & Time to make trip & 3.33 & 2.96 \\
\hline SQ32 & Convenience of routes (combined SQ 6 \& 18) & 3.70 & 3.29 \\
\hline SQ34 & Cleanliness/comfort (combined SQ 20 \& 21) & 3.93 & 3.48 \\
\hline SQ05 & Frequency of Service & 3.10 & 2.65 \\
\hline SQ15 & Latest weekdays & 3.01 & 2.56 \\
\hline SQ08 & Ease of transfer & 3.38 & 2.91 \\
\hline SQ13 & Ease to use schedule & 4.00 & 3.51 \\
\hline SQ11 & Value of fare/cost & 3.85 & 3.30 \\
\hline SQ22 & Safety at bus stop & 3.95 & 3.31 \\
\hline SQ24 & Safety getting off bus & 4.26 & 3.50 \\
\hline
\end{tabular}

With the exception of satisfaction with on-time performance, Members of 'Other' races, probably mainly Asians and native Americans, are significantly less satisfied with almost all elements of bus service than whites, Hispanics, and blacks. This may be partially a cultural phenomenon (i.e., a tendency to give lower ratings on surveys) but may also reflect lower level of service (i.e., less frequency, and later start / earlier end times) to neighborhoods predominantly populated by these racial groups. 
Gender

\begin{tabular}{|c|c|c|c|}
\hline & Table 9: JTA Gender & Male & Female \\
\hline Sample size & & $\mathbf{1 4 8 4}$ & $\mathbf{1 7 4 0}$ \\
\hline & & & \\
\hline SQ34 & Cleanliness/comfort (combined SQ 20 \& 21) & 3.60 & 3.37 \\
\hline SQ22 & Safety at bus stop & 3.58 & 3.32 \\
\hline SQ26 & Temperature in bus & 3.67 & 3.33 \\
\hline
\end{tabular}

Generally differences between female and male ratings of satisfaction are not significantly different for Jacksonville transit riders. The differences that are observed are noted above, dealing with cleanliness/comfort and safety at bus stops. Lower perception of safety at bus stops is not surprising - it is perhaps surprising that more safety issues were not rated differently by men and women. As it is, careful review of bus stop placements may help to reduce perceptions of danger among women. There may also be bus design characteristics that could be modified to better suit women. Qualitative investigation of these issues may help identify specific changes that can be made. 


\section{$\underline{\text { Recommendations }}$}

From the customer satisfaction model, it is possible to construct an "importanceperformance" matrix that graphically illustrates current bus riders' perceptions of JTA's operations.

\section{Importance / Performance Matrix Jacksonville Riders' perceptions of JTA service}

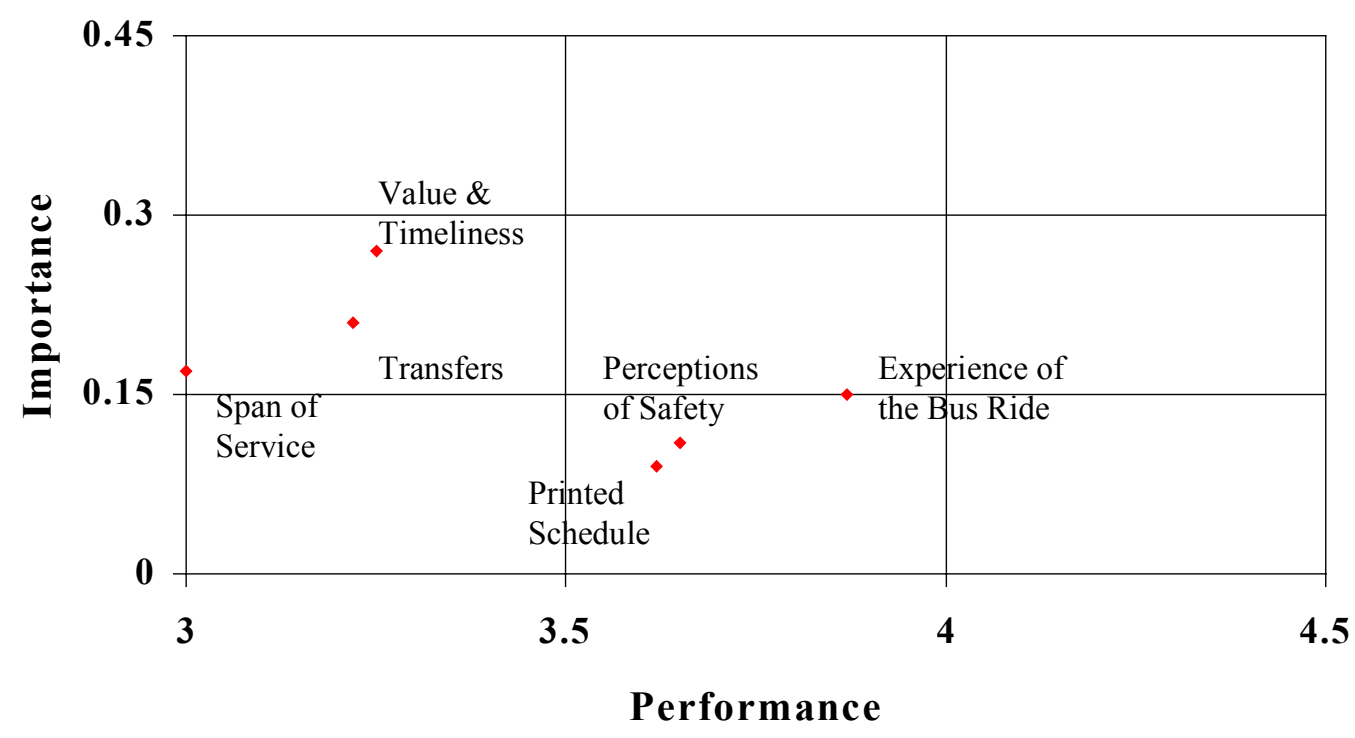

Figure 27: JTA Importance/Performance Matrix 
The chart has been divided into nine regions, reflecting various combinations of low, medium, and high performance and low, medium, and high importance. Borderline figures are interpreted as being in the higher of the importance categories they border on, but the lower of the performance categories. This provides the most conservative interpretation of the results. The interpretations of the chart regions are done as follows:

\begin{tabular}{|c|c|c|c|}
\hline \multicolumn{4}{|c|}{$\begin{array}{c}\text { Table } 10 \\
\text { Interpretations of JTA's Chart Regions }\end{array}$} \\
\hline \multicolumn{2}{|c|}{ Chart region } & \multirow[b]{2}{*}{ Interpretation } & \multirow[b]{2}{*}{ Areas } \\
\hline Importance & Performance & & \\
\hline Low & High & Possibly reduce focus on this area & \\
\hline Low & Medium & Maintain performance - no action & $\begin{array}{l}\text { Perceptions of } \\
\text { Safety, Printed } \\
\text { Schedule }\end{array}$ \\
\hline Low & Low & Maintain performance - no action & System Design \\
\hline Medium & High & Maintain performance - no action & \\
\hline Medium & Medium & Maintain performance - no action & $\begin{array}{l}\text { Experience of the } \\
\text { Bus Ride }\end{array}$ \\
\hline Medium & Low & Investigate for improvements & $\begin{array}{l}\text { Value \& } \\
\text { Timeliness, Span } \\
\text { of Service, } \\
\text { Transfers }\end{array}$ \\
\hline High & High & $\begin{array}{l}\text { Maintain performance - vigorous } \\
\text { quality checks, constant attention }\end{array}$ & \\
\hline High & Medium & Investigate for improvements & \\
\hline High & Low & Critical improvement area & \\
\hline
\end{tabular}


In JTA's case, the Perceptions of Safety, Experience of the Bus Ride, Printed Schedule, and System Design factors are all in the "maintain performance - no action" areas, while the Value and Timeliness, Span of Service, and Transfers factors fall into the "investigate for improvements" area.

The individual Value and Timeliness items that JTA scores particularly low on are:

\begin{tabular}{|ll|c|}
\hline \multirow{2}{*}{\multicolumn{1}{|c|}{ Item }} & \multicolumn{2}{c|}{ Scores } \\
\cline { 2 - 3 } & Index & Mean \\
\hline How Regularly Buses Arrive On Time & 92.98 & 3.07 \\
\hline Time to Make Trip & 85.08 & 3.17 \\
\hline Frequency of Service & 84.52 & 2.93 \\
\hline
\end{tabular}

The Span of Service items with low scores are:

\begin{tabular}{|lc|c|}
\hline \multirow{2}{*}{\multicolumn{1}{|c|}{ Item }} & \multicolumn{2}{c|}{ Scores } \\
\cline { 2 - 3 } & Index & Mean \\
\hline Latest Weekends & 86.50 & 2.68 \\
\hline Earliest Weekends & 85.20 & 2.94 \\
\hline Latest weekdays & 91.63 & 2.85 \\
\hline Frequency of Service & 84.52 & 2.93 \\
\hline
\end{tabular}

The Transfers items with low scores are:

\begin{tabular}{|ll|c|}
\hline \multirow{2}{*}{\multicolumn{1}{|c|}{ Item }} & \multicolumn{2}{c|}{ Scores } \\
\cline { 2 - 3 } & Index & Mean \\
\hline Number of Transfers & 87.84 & 3.18 \\
\hline Ease of Transfers & 82.34 & 3.21 \\
\hline Frequency of Service & 84.52 & 2.93 \\
\hline
\end{tabular}


JTA's customers are less satisfied with Span of Service than the other transit agencies' customers, as indicated by the index scores. This category's overall mean score indicates that Span of Service needs the most improvement. According to the individual mean scores, Jacksonville's customers are primarily unsatisfied with weekend service start and end times. The "latest weekends" item received the lowest mean, suggesting that weekend hours should be extended before instilling earlier start times. JTA's "frequency of service" achieved the lowest index score, indicating that its customers rated this item much lower than average. Therefore, resources should be dedicated to alleviating headway times, too.

Transfers could also use improvement. Since "frequency of service" is also a member of the Transfers category, it is appropriate to reinforce the need for shorter headway times. Also, the low scores associated with "number of transfers" and "ease of transfers" suggest that some resources should be devoted to acquire an easier transfer process (i.e. alleviate the number of transfers needed to cross town; reconsider transfer ticket policies). In contrast, the "ability to get where you are going" item suggests that JTA's passengers are satisfied with destinations reachable by bus.

Finally, Value/Timeliness indicates some areas that can be improved. In fact, this category showed the most impact on customer satisfaction, indicating that the greatest amount of resources should be devoted to the topics pertaining to this category. "Value of fare/cost" and "time to make trip" could use improvement. Moreover, the disappointing statistics in the "frequency of service" item is included in this category. In contrast, passengers were happy with the "how regularly buses arrive on time" and "temperature in bus" items located within the Value/Timeliness category. Studies should be conducted to determine the best way to decrease the time it takes to make a trip. Again, it is suggested that transfer policies should be revisited. Also, there may be a need for priority busing strategies (i.e. HOV lanes; express routes).

The matrix above suggests that "no action - continue existing strategies" would be a reasonable strategy for Safety items. However, Perceptions of Safety index scores are low, indicating that JTA should focus on improving performance in this area. Furthermore, demographic analysis suggests that women tend to have lower perceptions of safety at bus stops than men. In addition to the recommendations listed above, JTA should carefully review bus stop placements to ensure that perceptions of safety are not affected by bus stop placement. 
Satisfaction is not differentiated along ethnic lines. Hispanics are the most satisfied ethnic group, but whites and blacks (who make up over $90 \%$ of the rider population) are equal in most satisfaction areas.

Further demographic analysis shows that, unlike the overall income analysis of all Florida systems, Jacksonville's fare structure is perceived to be more valuable as income decreases. This may be due to the particular fare structure used by Jacksonville. It might be worthwhile to compare the fare structures among Florida transit systems in order to get a better understanding of why this correlation is different from the statewide statistics. 


\section{MCAT (1994)}

$\underline{\text { Factor Analysis }}$

Factor $1-\underline{\text { System Design }}$

Major loadings - $\quad$ SQ4 Hours of service

SQ3 Days of service

SQ5 Frequency of service

SQ18 Convenience of routes

SQ12 Ease to obtain schedule

Minor loading - $\quad$ SQ19 Dependability of buses (on time)

This construct includes customer satisfaction with hours of service, days of service, frequency of service and convenience of routes. The customers' "window" on system design, the ease of obtaining schedules, also loads on to this factor.

\begin{tabular}{|lc|c|}
\hline \multicolumn{2}{|c|}{ Table 11-I: MCAT 1994 Factor 1 - System Design } \\
\cline { 2 - 3 } & \multicolumn{2}{c|}{ Scores } \\
\cline { 2 - 3 } & Index & Mean \\
\hline Hours of Service & 105.01 & 3.81 \\
\hline Days of Service & 102.42 & 4.24 \\
\hline Frequency of Service & 111.17 & 3.85 \\
\hline Convenience of Routes & 101.73 & 4.06 \\
\hline Ease to Obtain Schedule & 105.75 & 4.33 \\
\hline Dependability of Buses & 101.27 & 3.95 \\
\hline Overall Mean & & $\mathbf{4 . 0 4}$ \\
\hline
\end{tabular}


Factor 5 - $\quad \underline{\text { Value }}$

Major loading - $\quad$ SQ11 Value of fare/cost

This construct is essentially comprised of the customer's perception of the value of fare.

\begin{tabular}{|c|c|c|}
\hline \multirow[b]{3}{*}{ Item } & or 5-1 & \\
\hline & \multicolumn{2}{|c|}{ Scores } \\
\hline & Index & Mean \\
\hline Value of Fare/Cost & 96.07 & 3.94 \\
\hline Overall Mean & & 3.94 \\
\hline
\end{tabular}

Factor 9 - Experience of the Bus Ride

Major loadings - $\quad$ SQ29 Driver courtesy

SQ21 Cleanliness/comfort

SQ25 Safety on bus and stops

Minor loading - $\quad$ SQ12 Ease to obtain schedule

The construct encompasses all the major experiential elements involved in the bus ride perceptions of the bus stop (cleanliness and comfort), meeting the driver, cleanliness and comfort of the bus itself, and whatever perceptions of safety one might have while riding. Ease of obtaining schedule slightly loads on to this factor as well.

\begin{tabular}{|c|c|c|}
\hline \multirow[b]{2}{*}{ Item } & \multicolumn{2}{|c|}{ Scores } \\
\hline & Index & Mean \\
\hline Driver Courtesy & 104.16 & 4.04 \\
\hline Cleanliness/Comfort & 99.52 & 4.31 \\
\hline Safety on Bus and Stops & 99.50 & 4.31 \\
\hline Ease to Obtain Schedule & 90.14 & 4.33 \\
\hline Overall Mean & & 4.36 \\
\hline
\end{tabular}


Factor 10 - Timeliness of Service

Major loadings - $\quad$ SQ19 Dependability of buses (on time)

SQ10 Time to make trip

Minor loadings - SQ18 Convenience of routes

SQ5 Frequency of service

This construct relates to issues of dependability of buses running on time and time to make trip. Also, frequency of service and convenience of routes were slightly loaded on to this factor. These variables are connected with the customer's ability to arrive at their destination in a timely manner.

\begin{tabular}{|lc|c|}
\hline \multicolumn{2}{|c|}{ Table 11-IV: MCAT 1994 Factor 10 - Timeliness of Service } \\
\hline \multicolumn{2}{|c|}{ Item } & Scores \\
\cline { 2 - 3 } & Index & Mean \\
\hline Dependability of Buses (On Time) & 101.27 & 3.95 \\
\hline Time to Make Trip & 105.33 & 3.92 \\
\hline Convenience of Routes & 101.73 & 4.06 \\
\hline Frequency of Service & 111.17 & 3.85 \\
\hline Overall Mean & & 3.95 \\
\hline
\end{tabular}




\section{$\underline{\text { Customer Satisfaction Model }}$}

The following chart indicates which of MCAT's factors were the most important in 1994. The most influential factors are those with the largest importance values. System Design, Timeliness, and Experience of the Bus Ride have the highest importance values.

\section{Customer Satisfaction Model: MCAT 1994}

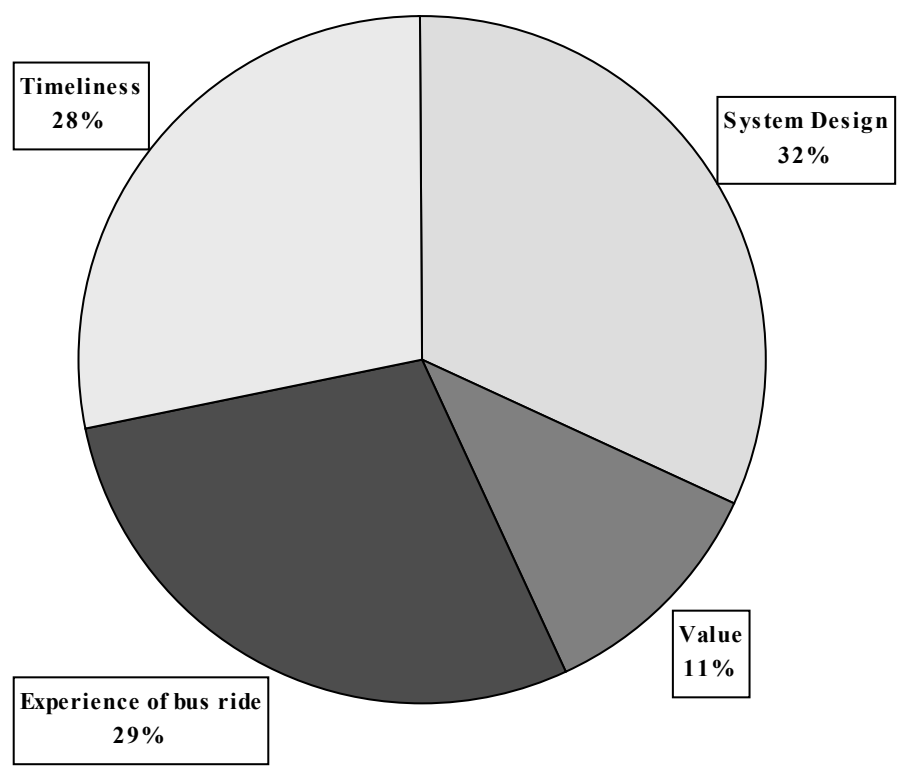

Figure 28: MCAT 1994 Customer Satisfaction Model 
From the customer satisfaction model, it is possible to construct an "importanceperformance" matrix that graphically illustrates current bus riders' perceptions of MCAT's operations in 1994.

\section{Importance / Performance Matrix Manatee County Riders' perceptions of MCAT service in 1994}

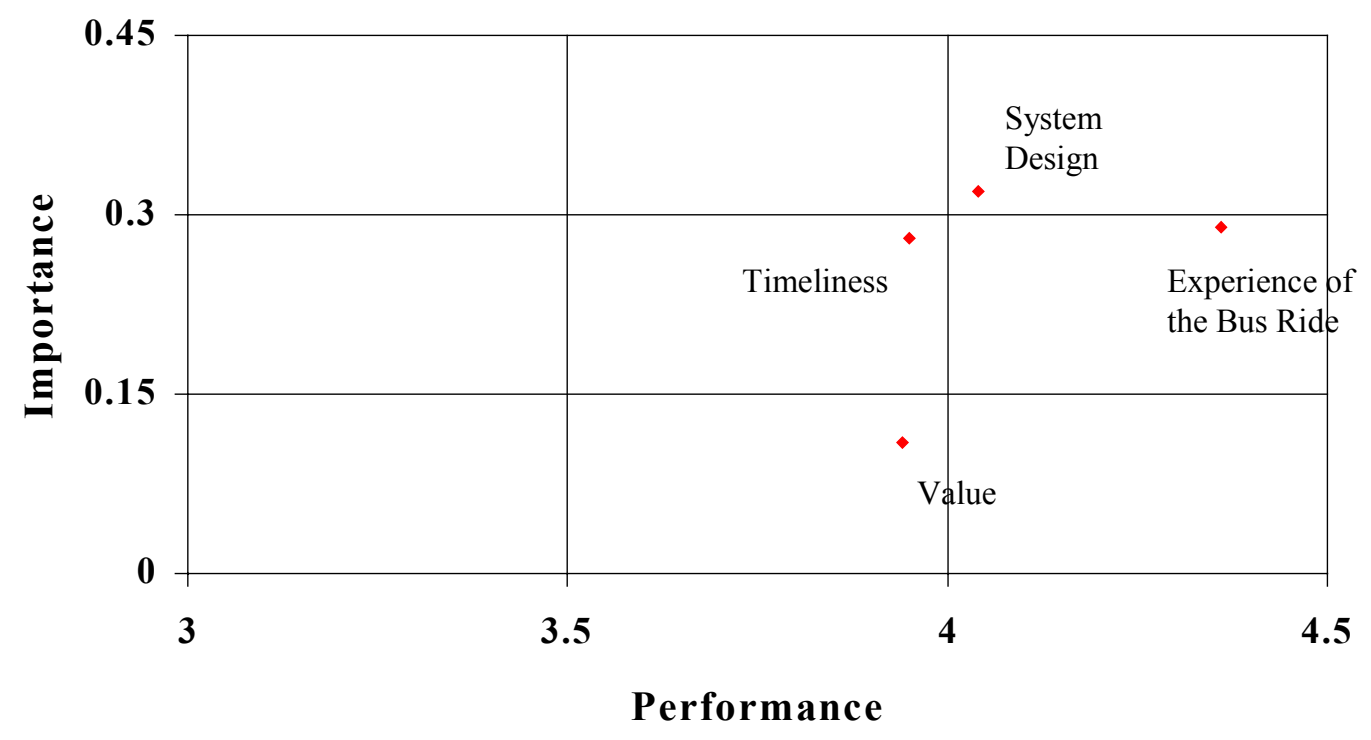

Figure 29: MCAT 1994 Importance/Performance Matrix

Due to the fact that there is a more recent survey for MCAT, a detailed explanation for these findings is not given. Rather, it can be used for comparative purposes to the findings for the 1998 survey.

For similar reasons, detailed demographic breakdowns are not given. Instead, the 1998 values are used for that purpose. 


\section{MCAT (1998)}

$\underline{\text { Factor Analysis }}$

Factor 1 - $\quad$ System Design

Major loadings - $\quad$ SQ4 Hours of service

SQ3 Days of service

SQ5 Frequency of service

SQ18 Convenience of routes

Minor loadings - $\quad$ SQ19 Dependability of buses

SQ10 Time to make trip

SQ8 Ease of transfer

This construct includes customer satisfaction with hours of service, days of service, frequency of service and convenience of routes.

\begin{tabular}{|lc|c|}
\hline \multicolumn{2}{|c|}{ Table 12-I: MCAT 1998 Factor 1 - System Design } \\
\cline { 2 - 3 } & \multicolumn{2}{c|}{ Scores } \\
\cline { 2 - 3 } Item & Index & Mean \\
\hline Hours of Service & 99.61 & 3.61 \\
\hline Days of Service & 96.75 & 4.01 \\
\hline Frequency of Service & 108.05 & 3.74 \\
\hline Convenience of Routes & 102.10 & 4.08 \\
\hline Dependability of Buses & 97.67 & 3.81 \\
\hline Time to Make Trip & 104.70 & 3.90 \\
\hline Ease of Transfer & 108.71 & 4.24 \\
\hline Overall Mean & & 3.91 \\
\hline
\end{tabular}


Factor 7 - $\quad$ Printed Schedules

Major loadings - $\quad$ SQ12 Ease to obtain schedule

SQ13 Ease to use schedule

Minor loading - $\quad$ SQ8 Ease of transfer

The construct relates to customer experience using schedules. The main variable in this construct is satisfaction with the ease of using the schedule. Predictably, other variables loading on this factor are ease to obtain schedule and how regularly the buses arrive on time.

\begin{tabular}{|lc|c|}
\hline \multicolumn{2}{|c|}{ Table 12-II: MCAT 1998 Factor 7 - Printed Schedules } \\
\hline \multicolumn{2}{c|}{ Scores } \\
\cline { 2 - 3 } Item & Index & Mean \\
\hline Ease to Obtain Schedules & 105.53 & 4.32 \\
\hline Ease to Use Schedules & 106.56 & 4.38 \\
\hline Ease of Transfer & 108.71 & 4.24 \\
\hline Overall Mean & & $\mathbf{4 . 3 2}$ \\
\hline
\end{tabular}


Factor 8 - $\quad \underline{\text { Value/Timeliness }}$

Major loadings - $\quad$ SQ11 Value of fare/cost

SQ10 Time to make trip

SQ19 Dependability of buses

Minor loadings - $\quad$ SQ18 Convenience of routes

SQ21 Cleanliness/comfort

This construct relates to customer satisfaction with value of fare/cost, time to make trip, and dependability of buses. In addition, convenience of routes and cleanliness/comfort also loaded slightly on to this factor. This is a shift from the 1994 survey, where value was a separate construct. In 1998, customers connect value more closely to the timeliness of service.

\begin{tabular}{|lc|c|}
\hline \multicolumn{2}{|c|}{ Table 12-III: MCAT 1998 Factor 8 - Value/Timeliness } \\
\cline { 2 - 3 } Item & Index & Mean \\
\cline { 2 - 3 } & 101.59 & 4.17 \\
\hline Value of Fare/Cost & 104.70 & 3.90 \\
\hline Time to Make Trip & 97.67 & 3.81 \\
\hline Dependability of Buses & 102.10 & 4.08 \\
\hline Convenience of Routes & 101.98 & 4.41 \\
\hline Cleanliness/Comfort & $\mathbf{4 . 0 7}$ \\
\hline \multirow{2}{*}{ Overall Mean } & \\
\hline
\end{tabular}


Factor 9 - Experience of the Bus Ride

Major loadings - $\quad$ SQ29 Driver courtesy

SQ25 Safety on bus and stops

SQ21 Cleanliness/comfort

SQ8 Ease of transfer

Minor loading - $\quad$ SQ13 Ease to use schedule

The construct encompasses all the major experiential elements involved in the bus ride perceptions of the bus stop (cleanliness and comfort), meeting the driver, cleanliness and comfort of the bus itself, and whatever perceptions of safety one might have while riding.

\begin{tabular}{|c|c|c|}
\hline \multirow[b]{2}{*}{ Item } & \multicolumn{2}{|c|}{ Scores } \\
\hline & Index & Mean \\
\hline Driver Courtesy & 102.78 & 4.43 \\
\hline Safety on Bus and Stops & 102.11 & 4.43 \\
\hline Cleanliness/Comfort & 101.98 & 4.41 \\
\hline Ease of Transfer & 108.71 & 4.24 \\
\hline Ease to Use Schedule & 106.56 & 4.38 \\
\hline Overall Mean & & 4.31 \\
\hline
\end{tabular}




\section{$\underline{\text { Customer Satisfaction Model }}$}

The following chart indicates which of MCAT's factors are the most important. The most influential factors are those with the largest importance values. Since System Design has the highest importance value, customer satisfaction relies upon this item the most. Therefore, MCAT should devote the most time and energy to this category in order to improve customer satisfaction ratings. By looking at the individual items in this construct, management can determine which items to focus on.

\section{Customer Satisfaction Model: MCAT 1998}

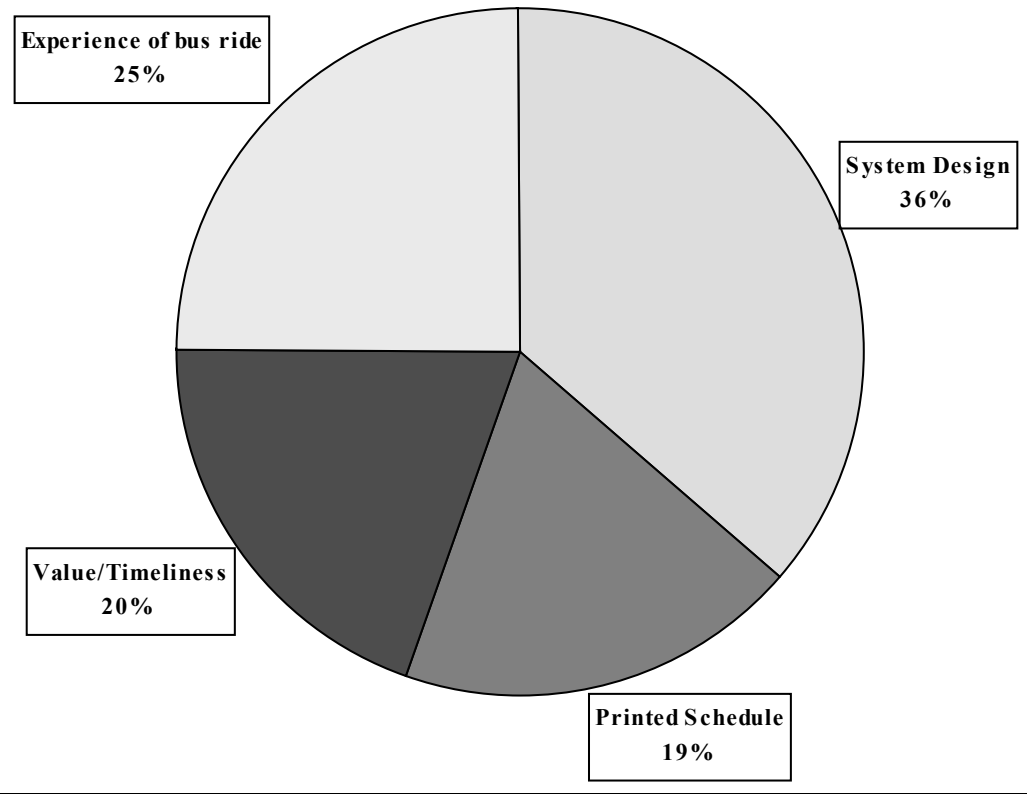

Figure 30: MCAT 1998 Customer Satisfaction Model 


\section{$\underline{\text { Demographic Analysis }}$}

\section{Income}

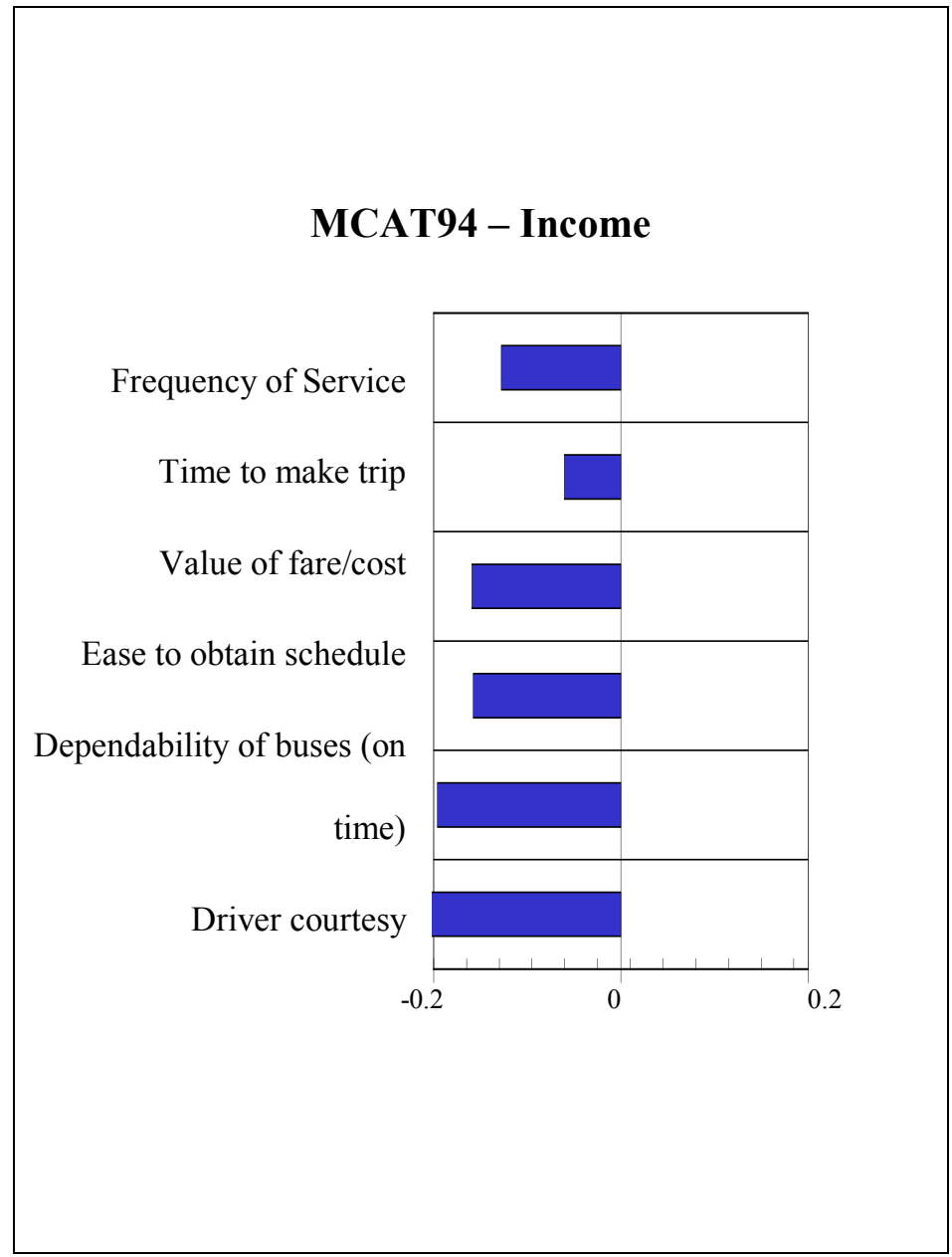

Figure 31: MCAT 1998 Correlation of income with satisfaction items

Higher income riders are more likely to be choice riders and hence will only use the bus when it is convenient for them. Thus those of higher income who are potential riders who feel that the bus is not convenient (in terms of routes, span of service, etc.) will choose not to ride the bus and would therefore not be sampled. Lower income riders do not have this choice. Therefore, a strong, positive correlation exists between income and time to make trip.

The items with which MCAT's lower income riders are more satisfied include ease of obtaining schedules, ease of using schedules, and bus dependability. Higher frequency of 
riding the bus probably makes lower income riders more aware of where to obtain schedules. Lower income riders also probably use this familiarity to set more realistic expectations for these elements of the bus-riding than higher income riders.

A positive correlation between income and driver courtesy satisfaction exists. Hence, higher income individuals tend to perceive this comfort factor to be more satisfactory. They rated driver courtesy higher than low-income riders. This probably relates to the fact that higher income individuals tend to ride buses in the nicer neighborhoods. Since roads that are located within high-income neighborhoods tend to be maintained better (i.e. fewer potholes), drivers may be more relaxed and friendly when encountering a wellmaintained stretch of road. 


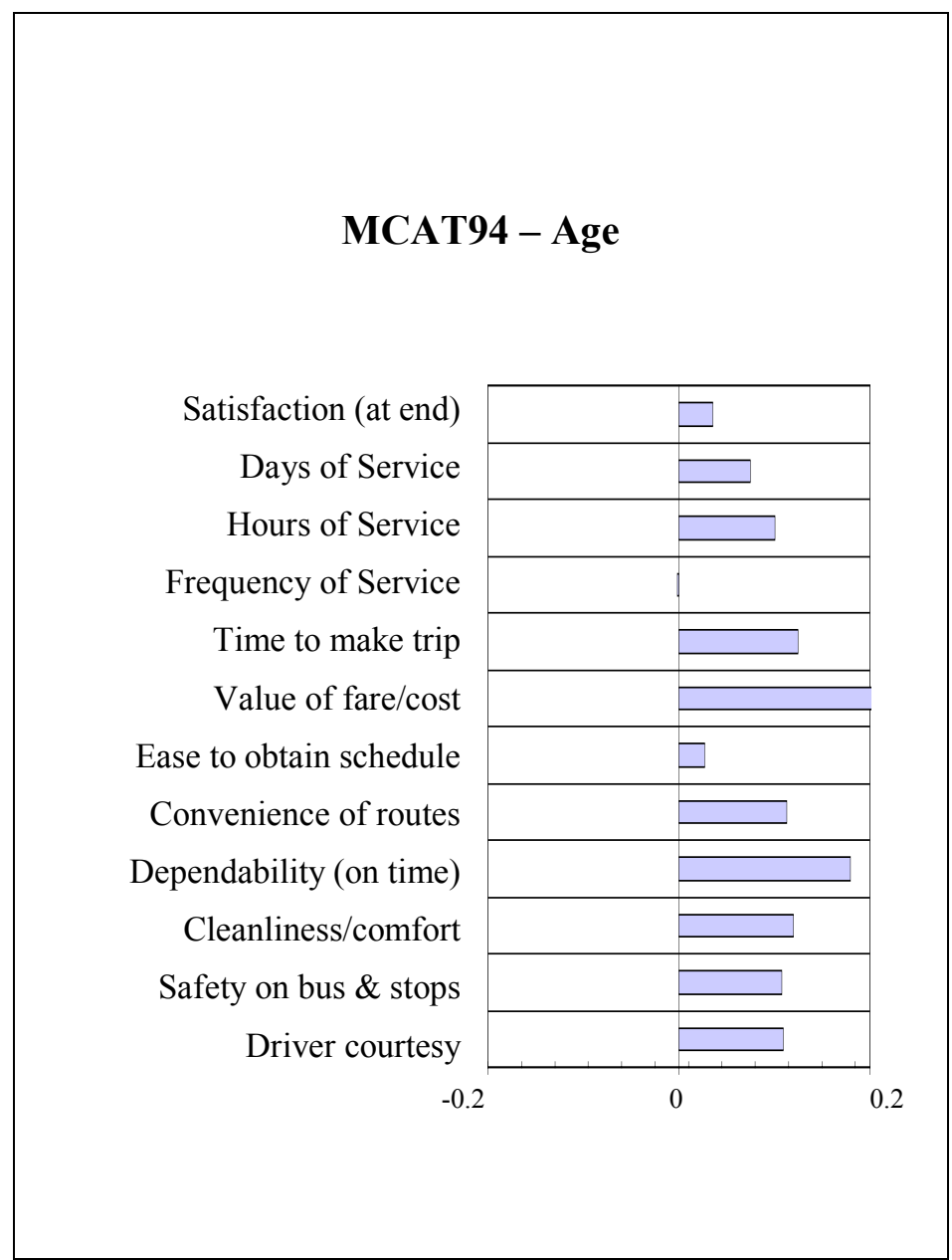

Figure 32: MCAT 1998 Correlation of age with satisfaction items

Almost all of the satisfaction factors are positively correlated with age, indicating older respondents to be more satisfied with transit service. Different experiences between the older and younger individuals may cause the satisfaction differences between younger and older individuals. Individual time constraints and amount of leisure time can influence a passenger's satisfaction level. For instance, younger people are usually on a tighter schedule, such as arriving to work on time. Therefore, younger people will usually be more conscious of time delays. This can lead younger people to be less satisfied with on-time performance. Moreover, younger people tend to be less satisfied with their time to make trip. 
A combination of familiarity with surroundings as well as non-peak trips can lead to higher satisfaction ratings. Elderly people tend to utilize transit services in their particular neighborhood. Therefore, they are usually more familiar with their surroundings. Those who are more familiar with their surroundings tend to be generally more comfortable. Familiarity can lead older people to bestow higher ratings to driver courtesy, ease to obtain schedule, ease to use schedule, and safety factors. Riding a bus during non-peak trips increases the tendency of passengers to be more satisfied with the cleanliness/comfort of bus. For example, a bus is perceived to be cleaner when there is less passenger congestion. Therefore, since older clients tend to ride the buses during non-peak service times, they tend to perceive the bus as being more clean/comfortable than younger passengers.

Fare structure causes passengers to rate the value of transit differently. Since Manatee County Area Transit's fare is half of the regular price for senior citizens over age 60, it is not surprising that the satisfaction with value increases with age. Moreover, MCAT's senior citizens may want more days of service, smoother transfers, and more convenient routes to take advantage of their discount fare status. 


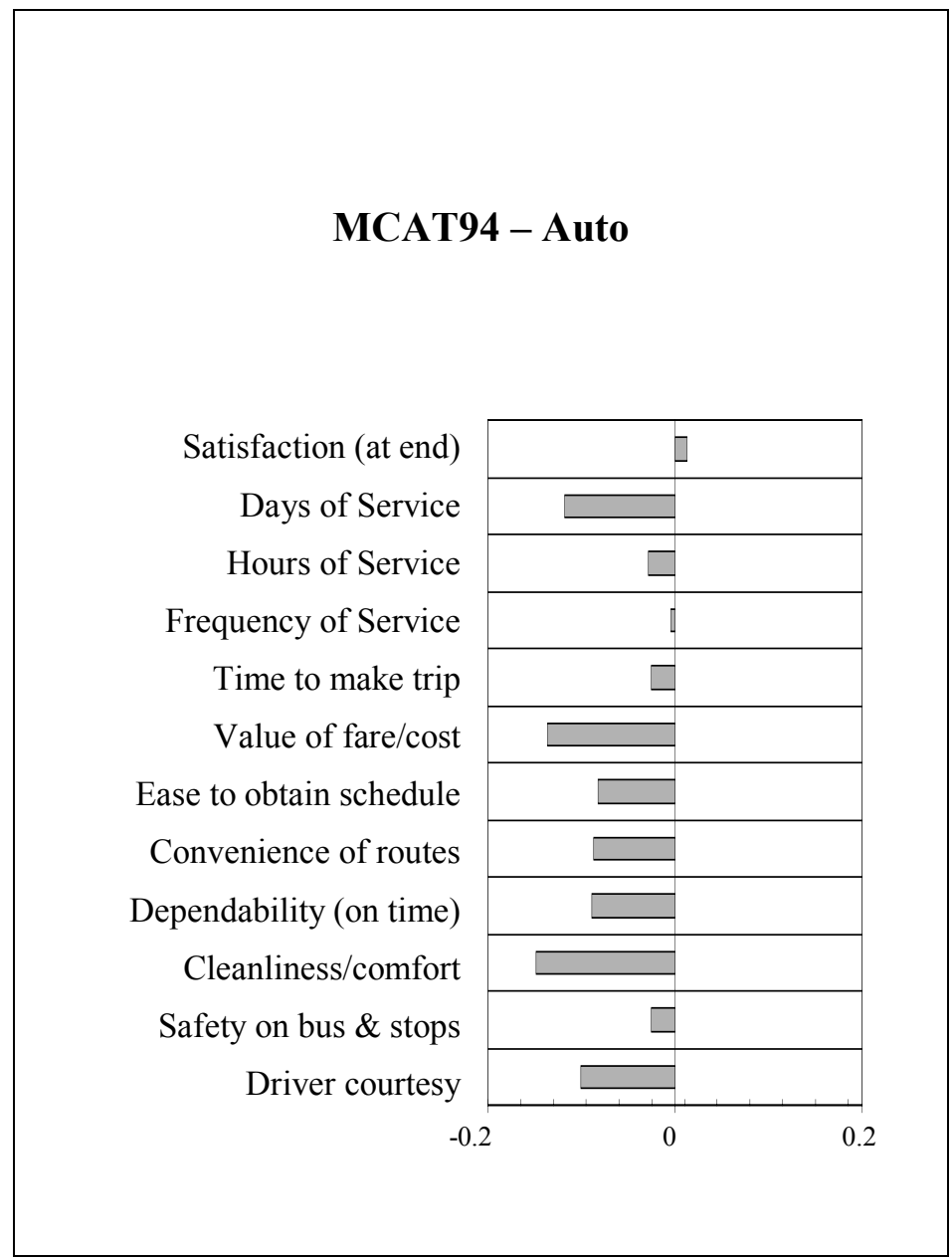

Figure 33: MCAT 1998 Correlation of auto ownership with satisfaction items

Overall, a positive correlation exists between number of automobiles and satisfaction with transit. Automobile owners ride transit only when it is convenient. Therefore, satisfaction with transit depends upon factors such as riding buses due to convenience versus riding them out of necessity. Since households that own automobiles have the luxury of 24-hour transportation, they are not hindered by transit schedules. In contrast, households without vehicles are constrained by transit service times, taxi costs, and/or friends' schedules. Therefore, persons without automobiles are less satisfied with days of service, hours of service, frequency of service, and convenience of routes.

Those who are choice riders may experience transit differently than non-choice riders. The positive satisfaction correlation between auto ownership and time to make trip 
suggests that auto owners ride the bus only when it is a convenience. Moreover, choice riders only choose the transit option if they feel safe, comfortable, and respected. Therefore, positive correlations between auto ownership and factors such as driver courtesy and safety are understandable.

Familiarity with transit service may also cause negative correlations between automobile ownership and transit satisfaction. For instance, ease to obtain schedule, ease to use schedule, ease of transfer, and dependability of buses are all issues that are negatively correlated with ownership. Much can be explained by the fact that those who do not have cars use transit the most. Therefore, familiarity with bus schedules and bus routes will lead persons without cars to be more satisfied. A negative correlation between ease of transfer and ownership suggests that since the non-owners frequently depend upon bus transfers, they are used to the routine method and therefore perceive it as easier. Also, the riders who use transit the most will have a more realistic view of bus dependability and allow for more time to make trip.

Unlike the All Systems results, satisfaction of the value of fare/cost is positively correlated with number of automobiles in household. Value of fare/cost was perceived to be more satisfying by those with vehicles. Assuming passengers who own cars receive a larger income, the fare would be a smaller percentage of take home salary for them. Also, those with vehicles know the true cost of providing transportation and would thereby consider transit service a better buy than those without a vehicle. 


\section{Ethnicity}

\begin{tabular}{|c|c|c|c|}
\hline & Table 13: MCAT 1998 Ethnicity & White & Black \\
\hline Sample size & & $\mathbf{7 9 8}$ & $\mathbf{1 6 8}$ \\
\hline & & & \\
\hline SQ13 & Ease to use schedule & 4.49 & 4.24 \\
\hline SQ10 & Time to make trip & 4.01 & 3.75 \\
\hline SQ11 & Value of fare/cost & 4.30 & 4.00 \\
\hline SQ33 & Dependability of buses (on time) (combined SQ 9 \& 19) & 3.99 & 3.49 \\
\hline
\end{tabular}

Whites are more satisfied than blacks with ease of using schedule, time to make trip, value of fare, and dependability of buses. There seems to be a serious deficiency that relates to scheduling. If the schedule is hard to understand, it is not surprising that value, time to make trip, and dependability ratings are all lower. An investigation into reasons why the schedule is considered hard to use is in order.

\section{Gender}

\begin{tabular}{|c|c|c|c|}
\hline & Table 14: MCAT 1998 Gender & Male & Female \\
\hline Sample size & & $\mathbf{2 2 2}$ & $\mathbf{2 8 5}$ \\
\hline & & & \\
\hline SQ05 & Frequency of Service & 3.82 & 3.62 \\
\hline SQ04 & Hours of Service & 3.71 & 3.47 \\
\hline
\end{tabular}

Men are more satisfied than women with frequency of service and hours of service. This may be due to men being more likely to be choice riders than women, due to wage differences and other factors. If women are more dependent on the bus for all their transportation needs, it would be logical if they were less satisfied with hours of service and frequency of service. 


\section{$\underline{\text { Recommendations }}$}

From the customer satisfaction model, it is possible to construct an "importanceperformance" matrix that graphically illustrates current bus riders' perceptions of MCAT's operations.

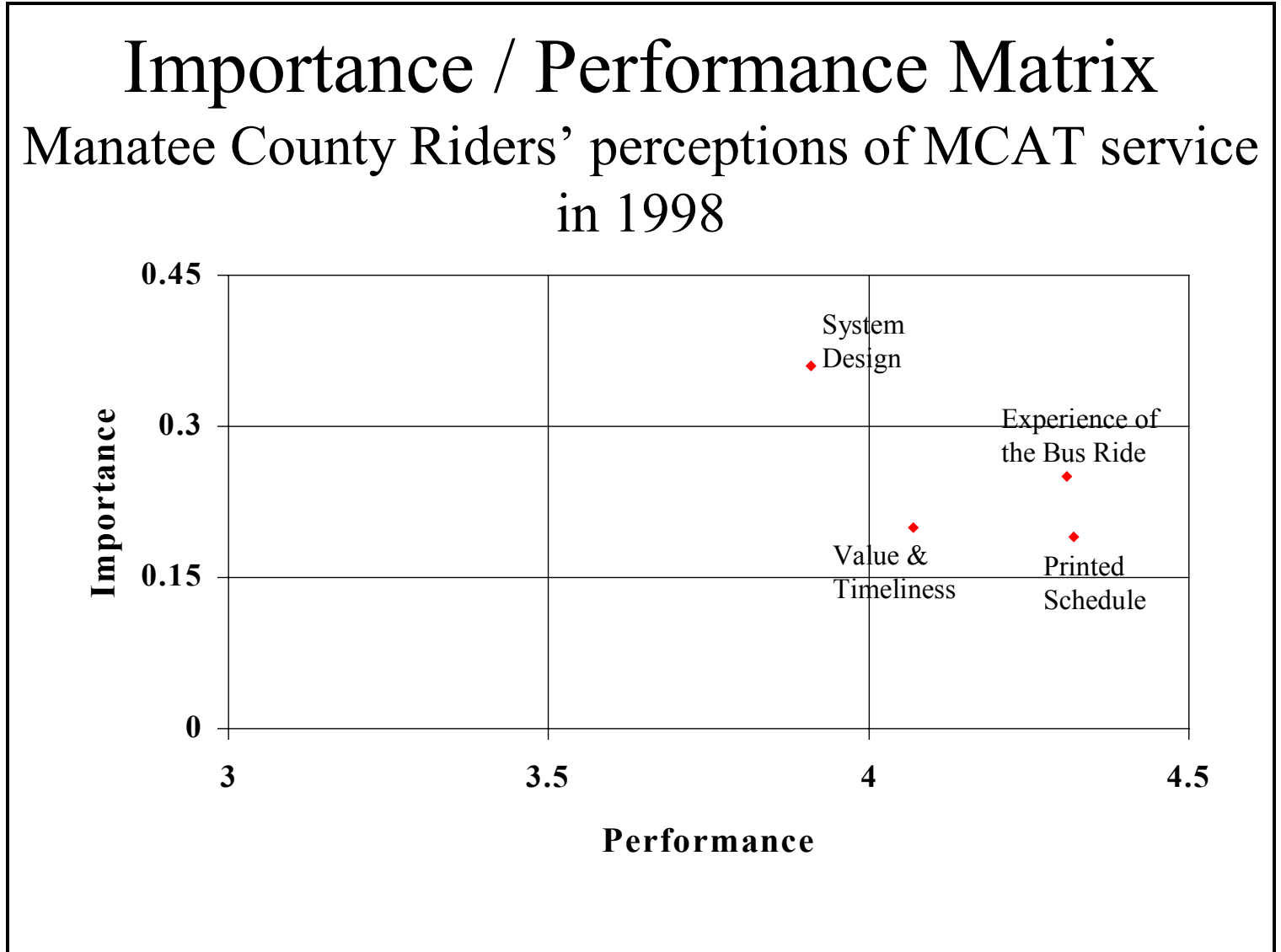

Figure 34: MCAT 1998 Importance/performance Matrix 
The chart has been divided into nine regions, reflecting various combinations of low, medium, and high performance and low, medium, and high importance. Borderline figures are interpreted as being in the higher of the importance categories they border on, but the lower of the performance categories. This provides the most conservative interpretation of the results. The interpretations of the chart regions are done as follows:

\begin{tabular}{|c|c|c|c|}
\hline \multicolumn{4}{|c|}{$\begin{array}{c}\text { Table 15 } \\
\text { Interpretations of MCAT's Chart Regions }\end{array}$} \\
\hline \multicolumn{2}{|c|}{ Chart region } & \multirow[b]{2}{*}{ Interpretation } & \multirow[b]{2}{*}{ Areas } \\
\hline Importance & Performance & & \\
\hline Low & High & Possibly reduce focus on this area & \\
\hline Low & Medium & Maintain performance - no action & \\
\hline Low & Low & Maintain performance - no action & \\
\hline Medium & High & Maintain performance - no action & $\begin{array}{l}\text { Experience of the } \\
\text { Bus ride, } \\
\text { Value/Timeliness, } \\
\text { Printed Schedule }\end{array}$ \\
\hline Medium & Medium & Maintain performance - no action & \\
\hline Medium & Low & Investigate for improvements & \\
\hline High & High & $\begin{array}{l}\text { Maintain performance - vigorous } \\
\text { quality checks, constant attention }\end{array}$ & \\
\hline High & Medium & Investigate for improvements & System Design \\
\hline High & Low & Critical improvement area & \\
\hline
\end{tabular}

A comparison to the 1994 survey shows that the performance rating on System Design has slipped slightly, but that performance on other factors has improved. This suggests 
that current focus should be on System Design issues. This is the only factor in the model that falls into the category of 'Investigate for improvements.'

The individual System Design items that MCAT scores relatively low on are:

\begin{tabular}{|lc|c|}
\hline \multirow{2}{*}{\multicolumn{1}{|c|}{ Item }} & \multicolumn{2}{c|}{ Scores } \\
\cline { 2 - 3 } & Index & Mean \\
\hline Hours of Service & 99.61 & 3.61 \\
\hline Frequency of Service & 108.05 & 3.74 \\
\hline Dependability of Buses & 97.67 & 3.81 \\
\hline
\end{tabular}

Manatee is definitely doing a good job with respect to the System Design category. For instance, all of the items included in this construct are nearly average if not better than average. This suggests that MCAT's customers are more satisfied than the other Florida transit customers surveyed. The only two areas that may need a bit of tweaking are "hours of service" and "frequency of service". Perhaps some new buses could be added to the line to increase frequency. Moreover, if resources are utilized to increase service hours, customers may become satisfied further.

The overall means of each of the factors also indicate that MCAT is fulfilling the needs of its passengers. The major categories of Schedules, Value/Timeliness, and Experience of the Bus Ride revealed high satisfaction ratings and also suggest that MCAT is doing a better than average job at fulfilling its clients' expectations. MCAT's overall recommendation is to continue providing exemplary service.

Most of the demographic correlations with satisfaction are related to the level of choice ridership in demographic groups defined by income, age, and auto ownership. Higher income groups tend to be more satisfied due to their higher level of choice in using transit. No specific recommendations for changes to MCAT service arose from the demographic analysis.

Small sample sizes did not permit a thorough demographic analysis of other demographic categories (such as race and gender) for MCAT's operations. 


\section{PalmTran}

$\underline{\text { Factor Analysis }}$

Factor 1 - $\quad$ System Design

Major loadings - $\quad$ SQ7 Number of transfers

SQ8 Ease of transfers

SQ5 Frequency of service

SQ6 Ability to get where you want to go

SQ9 How regularly buses arrive on time

SQ10 Time to make trip

This construct includes customer satisfaction with ease of transfers and number of transfers, frequency of service and convenience of routes, on-time performance and time to make trip. The emphasis is on transfers, indicating that transfers are a major element in the overall satisfaction with the design of the system in filling customer travel needs.

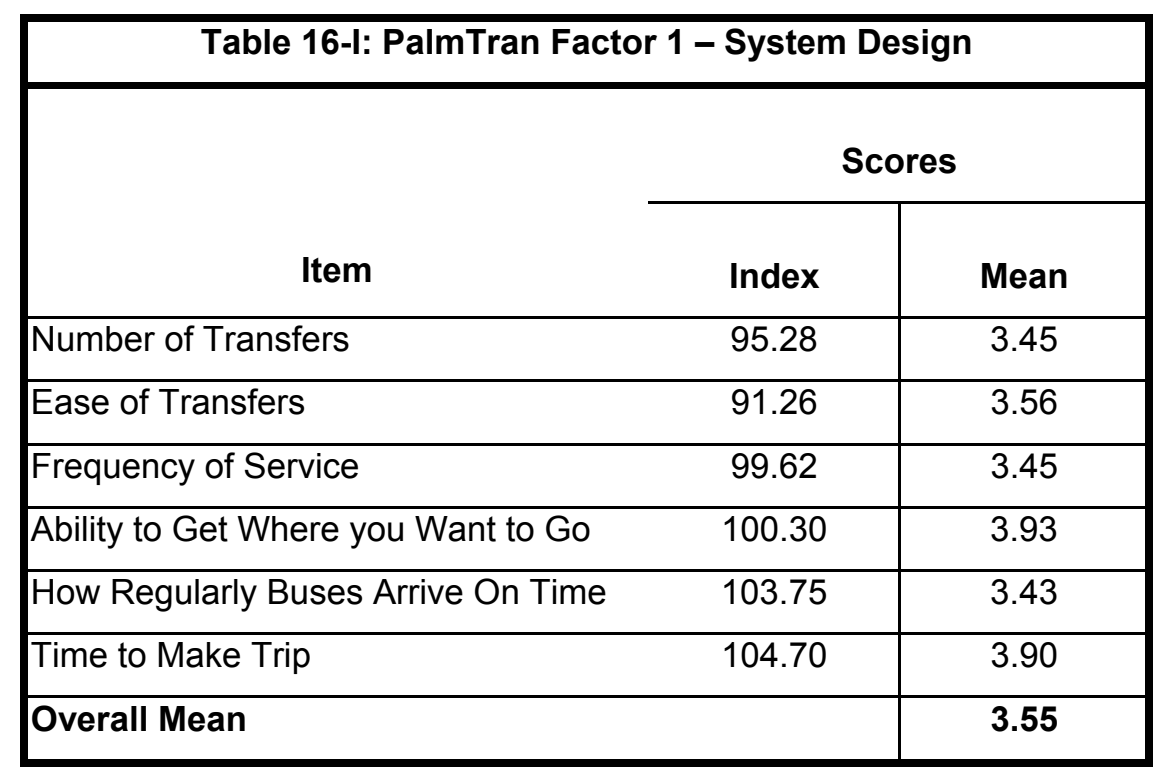


Factor 2 - $\quad$ Span of Service

Major loadings - $\quad$ SQ17 Latest weekends

SQ16 Earliest weekends

SQ15 Latest weekdays

SQ14 Earliest weekdays

This construct relates to customer satisfaction with span of transit service availability.

The components are satisfaction with earliest weekday, latest weekday, earliest weekend day, and latest weekend day service. Frequency of service also slightly loaded on this factor.

\begin{tabular}{|lc|c|}
\hline \multicolumn{2}{|c|}{ Table 16-II: PalmTran Factor 2 - Span of Service } \\
\cline { 2 - 3 } & \multicolumn{2}{c|}{ Scores } \\
\cline { 2 - 3 } \multicolumn{1}{|c|}{ Item } & Index & Mean \\
\hline Latest weekdays & 99.03 & 3.07 \\
\hline Latest weekends & 97.25 & 3.02 \\
\hline Earliest weekends & 94.99 & 3.28 \\
\hline Earliest weekdays & 96.86 & 3.63 \\
\hline Overall Mean & & 3.25 \\
\hline
\end{tabular}


Factor 3 - Perceptions of Safety

Major loadings - $\quad$ SQ22 Safety at bus stop

SQ23 Safety on bus

SQ24 Safety getting off bus

SQ20 Clean buses and stop

This construct relates to customer satisfaction with safety at bus stop, safety getting off bus, clean buses and stops, safety on bus, as well as temperature on bus. The variable availability of seats also slightly loaded on this factor. The connection between safety and cleanliness requires some explanation. One possibility is that the passenger construct relates to the apparent level of concern and respect the transit agency has for their passengers. Cleanliness is a very common way of showing respect and concern for customers - in restaurants, retail businesses, even in personal interactions. One of the correlates of transit agency concern for customers could be safety at bus stops, since the perception that the agency is taking care of its equipment and facilities may transfer over into perceptions of safety.

\begin{tabular}{|lc|c|}
\hline \multicolumn{2}{|c|}{ Table 16-III: PalmTran Factor 3 - Perceptions of Safety } \\
\hline \multicolumn{2}{|c|}{ Scores } \\
\cline { 2 - 3 } \multicolumn{1}{|c|}{ Item } & Index & Mean \\
\hline Safety on Bus Stop & 97.25 & 3.81 \\
\hline Safety on Bus & 97.41 & 4.04 \\
\hline Safety Getting Off Bus & 96.67 & 3.92 \\
\hline Clean Buses and Stop & 98.86 & 3.73 \\
\hline Overall Mean & & 3.87 \\
\hline
\end{tabular}


Factor 5 - $\quad$ Value

Major loading - $\quad$ SQ11 Value of fare/cost

Minor loadings - $\quad$ SQ12 Ease to obtain schedule

SQ10 Time to make trip

SQ9 How regularly buses run on time

The primary component of this construct is customer perception of value. Interestingly, ease of obtaining schedules also loads strongly on to this factor. This indicates that customers' perception of value is highly related to their ability to obtain a schedule. As a result, it may be advantageous to make schedules more easily accessible to customers.

The presence of the variables Time to make trip and buses running on time in this construct indicate that to a large extent, the time involved in the trip may be more of an issue than the cost of the fare.

\begin{tabular}{|lc|c|}
\hline \multicolumn{2}{|c|}{ Table 16-IV: PalmTran Factor 5 - Value } \\
\cline { 2 - 3 } & \multicolumn{2}{c|}{ Scores } \\
\cline { 2 - 3 } Item & Index & Mean \\
\hline Value of Fare/Cost & 98.60 & 4.05 \\
\hline Ease to Obtain Schedule & 90.67 & 3.71 \\
\hline Time to Make Trip & 93.98 & 3.50 \\
\hline How Regularly Buses Run On Time & 103.75 & 3.43 \\
\hline Overall Mean & & 3.67 \\
\hline
\end{tabular}


Factor 6 - Comfort of Ride

Major loadings - $\quad$ SQ27 Availability of seats

SQ26 Temperature in bus

This construct is focused entirely on physical ride comfort, being comprised of the variables availability of seats and temperature in the bus.

\begin{tabular}{|lc|c|}
\hline \multicolumn{2}{|c|}{ Table 16-V: PalmTran Factor 6 - Comfort of Ride } \\
\hline \multirow{2}{*}{ Item } & Index & Mean \\
\cline { 2 - 3 } & 101.00 & 3.94 \\
\hline Availability of Seats & 102.88 & 3.96 \\
\hline Temperature in Bus & 3.95 \\
\hline Overall Mean & & \multicolumn{2}{c|}{ Scores } \\
\hline
\end{tabular}

Factor $7-\underline{\text { Schedule }}$

Major loadings - $\quad$ SQ12 Ease to obtain schedule

SQ13 Ease to use schedule

The construct relates to customer experience using schedules. The main variables in this construct are satisfaction with the ease of using the schedule and ease of obtaining schedule.

\begin{tabular}{|lc|c|}
\hline \multicolumn{2}{|c|}{ Table 16-VI: PalmTran Factor 7 - Schedule } \\
\cline { 2 - 3 } & \multicolumn{2}{c|}{ Scores } \\
\cline { 2 - 3 } Item & Index & Mean \\
\hline Ease to Obtain Schedule & 90.67 & 3.71 \\
\hline Ease to Use Schedule & 94.23 & 3.88 \\
\hline Overall Mean & & 3.79 \\
\hline
\end{tabular}


Factor 11 - Driver

Major loadings - $\quad$ SQ29 Driver courtesy

SQ28 Ability to drive

Minor Loading - $\quad$ SQ13 Ease of using schedule

This construct relates to customer satisfaction with bus driver performance. The two main variables are driver courtesy and ability to drive the bus. Ease of using schedules loads slightly on to this factor, indicating that the bus driver is an important source for assisting customers with schedule information.

\begin{tabular}{|lc|c|}
\hline \multicolumn{2}{|c|}{ Table 16-VII: PalmTran Factor 11 - Driver } \\
\cline { 2 - 3 } & \multicolumn{2}{c|}{ Scores } \\
\cline { 2 - 3 } & Indem & Mean \\
\hline Driver Courtesy & 96.45 & 4.16 \\
\hline Ability to Drive & 99.07 & 4.25 \\
\hline Ease of Using Schedule & 94.23 & 3.88 \\
\hline Overall Mean & & $\mathbf{4 . 0 9}$ \\
\hline
\end{tabular}




\section{$\underline{\text { Customer Satisfaction Model }}$}

The following chart indicates which of PalmTran's factors are the most important. The most influential factors are those with the largest importance values. For instance, since System Design, Perceptions of Safety, and Driver have the highest importance values, PalmTran should devote the most time and energy to these categories in order to improve customer satisfaction ratings. By looking at the individual items in each of these three constructs, management can determine which items to focus on.

\section{Customer Satisfaction Model: PalmTran}

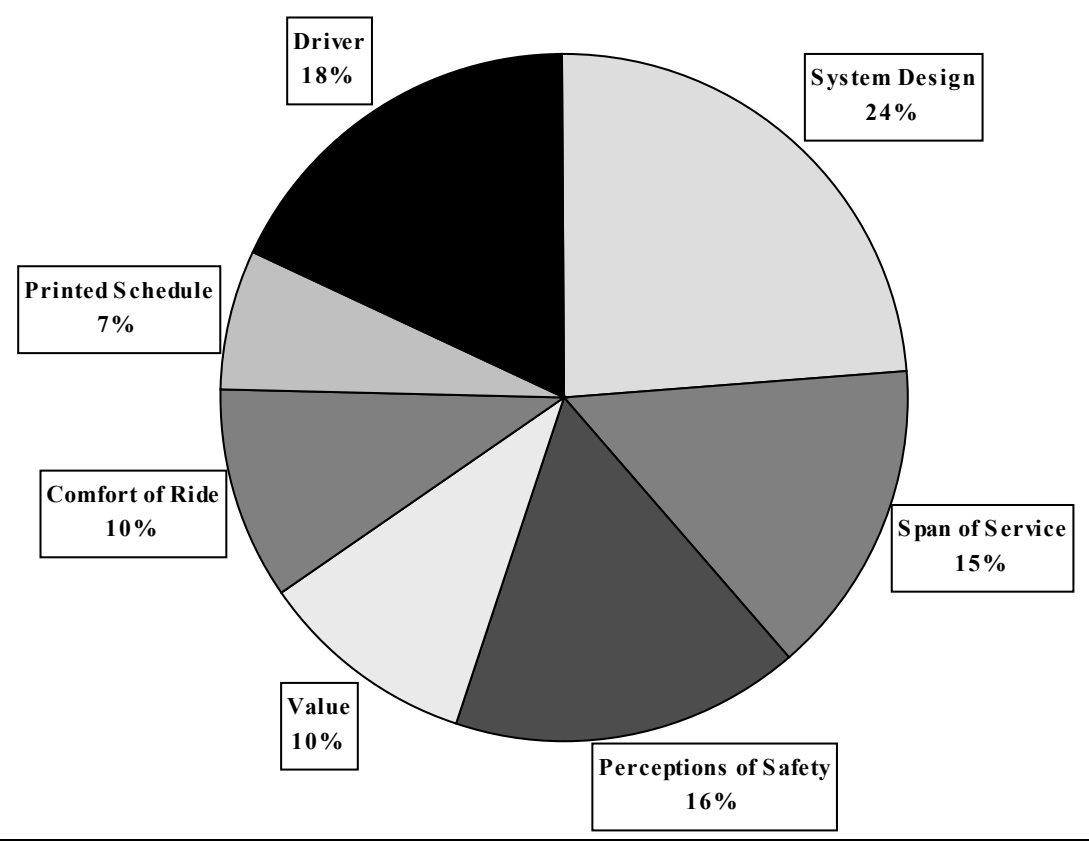

Figure 35: PalmTran Customer Satisfaction Model 
$\underline{\text { Demographic Analysis }}$

Income

PALMTRAN - Income

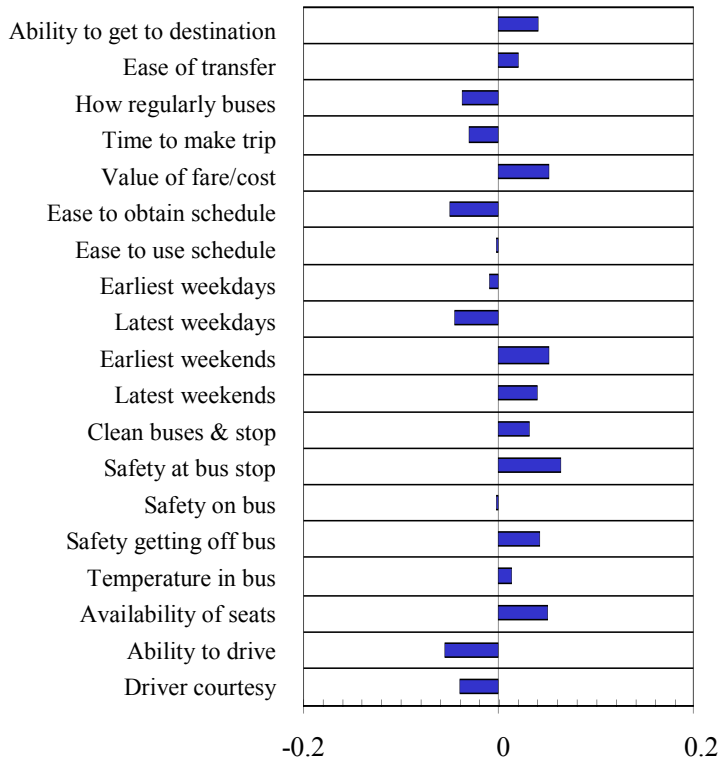

Figure 36: PalmTran Correlation of income with satisfaction items

According to Palm Tran's survey results, those with a higher income tend to be more satisfied with comfort factors, weekend service, bus stop locations, and fare value. The higher income individuals may experience transit differently than lower income individuals. For instance, the buses that higher income passengers utilize might be less crowded. Also, bus stops near higher income residences tend to be safer. It is therefore not surprising that higher income individuals related greater satisfaction for factors such as clean buses and stop, safety at bus stop, safety getting off bus, availability of seats, and temperature in bus. 
Choice riders perceive transit service differently. Since choice riders utilize transit only when it is convenient, they are inclined to be more satisfied with measures such as ability to get where and ease of transfer. Moreover, assuming higher income individuals have access to other forms of transportation, they tend to be more satisfied with weekend service start and end times. The higher income groups rate Palm Tran's fare as being more valuable. This can be derived from a combination of circumstances (such as fare as a percentage of income and riding the bus only when it is convenient).

Lower income individuals usually ride transit more often. Therefore, they tend to be more familiar with bus routes, schedules, and drivers. The implication of familiarity is that lower income individuals are inclined to be more satisfied with bus regularity, time to make trip, ease to obtain schedule, ability to drive, and driver courtesy. 
PALMTRAN - Age

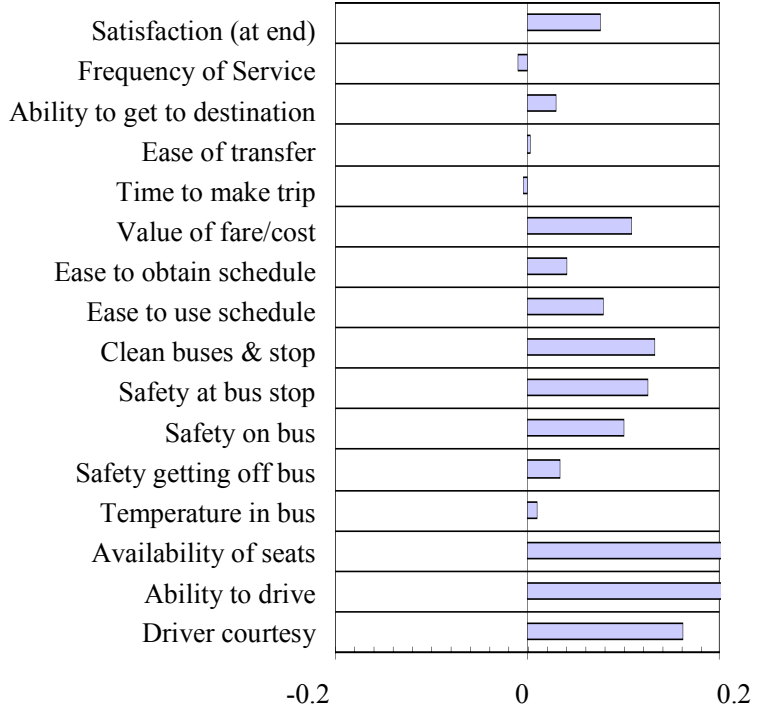

Figure 37: PalmTran Correlation of age with satisfaction items

An analysis of a non-linearly related item, satisfaction with number of transfers, showed that those aged 25-59 were significantly less satisfied than all other groups with number of transfers.

Older passengers seem to be more satisfied with transit than younger individuals. Almost all satisfaction measures have a positive correlation with age. A combination of senior citizen fares, familiar neighborhoods, frequent travel on the same routes, and non-peak trips yield higher satisfaction ratings. For instance, Palm Tran's senior citizen fare is half of the regular fare price. Therefore, it is no surprise that older adults rate transit value higher. 
Older passengers usually stay within the boundaries of their neighborhood. Therefore, they tend to rate bus stop cleanliness and safety better. Also, older passengers are usually more satisfied with the current stops in their neighborhood and would therefore rate "ability to get where they want to go" better than younger riders. Positive correlations between age and ease to obtain schedule, ease to use schedule, ability to drive, and driver courtesy can occur due to frequent travel on the same routes. Moreover, if travel is during non-peak hours, individuals may be more satisfied with safety on bus, availability of seats, and temperature in bus measures. 


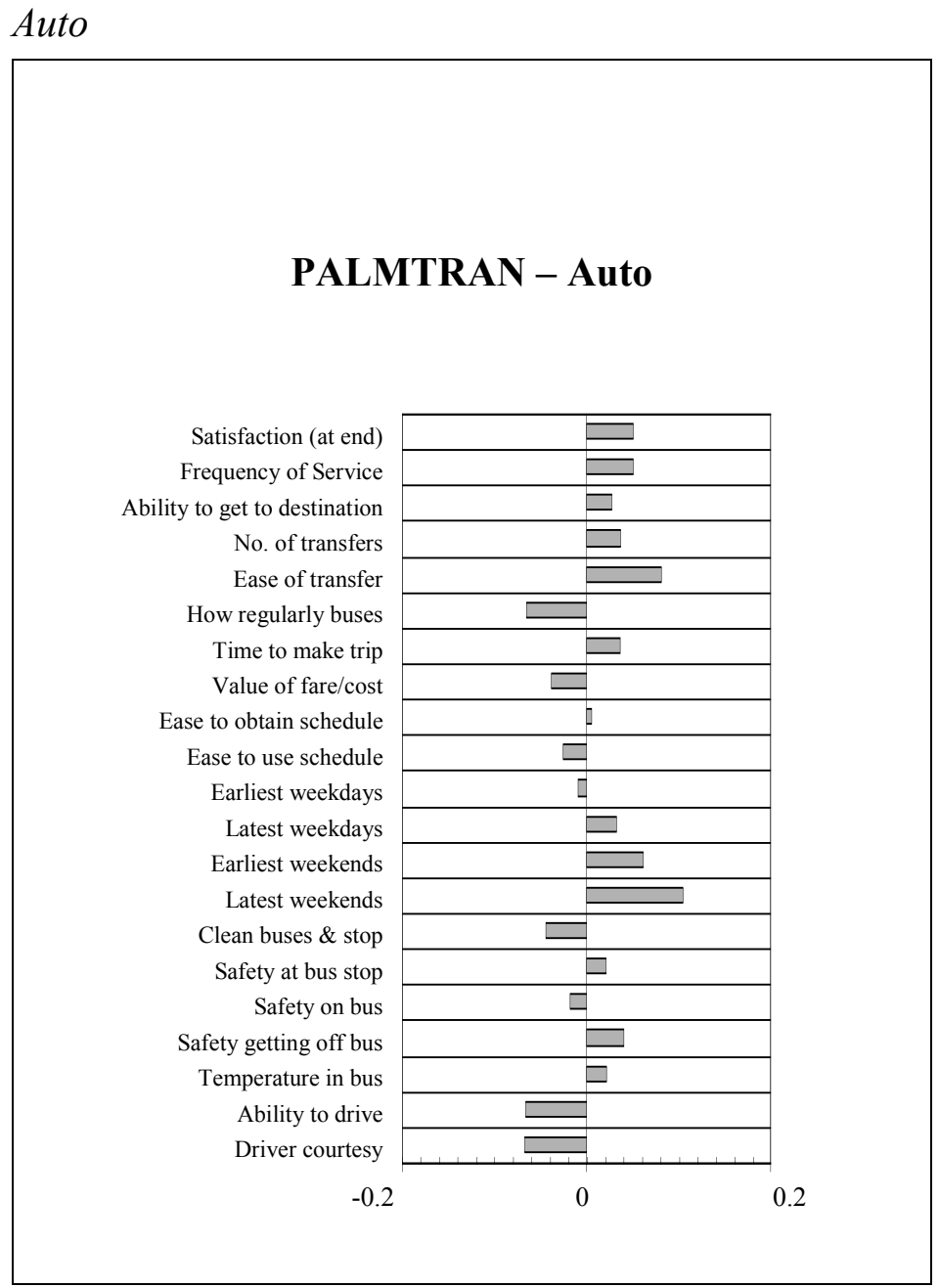

Figure 38: PalmTran Correlation of auto ownership with satisfaction items

An analysis of a non-linearly related item, availability of seats, showed that those with 3 vehicles in the household were significantly less satisfied with availability of seats than all other groups.

Auto ownership impacts these satisfaction levels because there is a difference between riding buses due to convenience versus riding them out of necessity. Overall, a positive correlation exists between number of automobiles and satisfaction. For the most part, auto owners choose to ride transit when it is convenient. Since auto owners only ride transit when it is convenient, they are more likely to highly rate factors such as frequency of service, ability to get where, number of transfers, ease of transfer, time to make trip, and weekend service times. In contrast, those who depend on autos for daily transportation needs may encounter difficulties rearranging their schedules to coincide 
with transit availability. Also, their destinations may not be on fixed-route routes. Consequently, riders without transportation alternatives may have to utilize destinations that are not their first choice.

Those who own automobiles tend to be more satisfied with Palm Tran's transit service begin and end times. Households that own automobiles have the luxury of 24-hour transportation. In contrast, households without vehicles are constrained by transit service times, taxi costs, and/or friends' schedules. Therefore, persons without automobiles are less satisfied with transit service begin and end times.

As car ownership increases, satisfaction for number of transfers and temperature in bus increases. The positive relationship between car ownership and satisfaction reveals a car owner's tendency to utilize transit for short trips. In contrast, those without cars rely on transit for all destinations. Usually, longer trips require more transfers, which can be seen as cumbersome by those who use transit as their only reliable form of transportation. Moreover, auto owners are more likely to ride buses on less crowded routes (i.e. Park-NRide). Less crowded buses are usually more comfortable since the temperature inside can be regulated better (i.e. less body heat). Therefore, auto owners tend to rate temperature in bus as more satisfactory.

Satisfaction of the value of fare/cost is negatively correlated with number of automobiles in household. Those without cars perceive transit as more valuable because they utilize it for all types of trips. Without transit, these passengers would not be able to work, go to school, run errands, and/or utilize public facilities. In contrast, customers who own automobiles tend to underestimate the value of transit. Automobile owners do not know the difficulties associated with trying to obtain rides for errands when transit is not available.

Familiarity with transit service may also cause negative correlations between automobile ownership and transit satisfaction. For instance, ease of using schedule, driver courtesy, dependability of buses and safety on bus are all issues that are negatively correlated with ownership. Much can be explained by the fact that those who do not have cars use transit the most. Therefore, familiarity with bus schedules and bus routes will lead persons without cars to be more satisfied. Those who use transit often will also be more familiar with bus drivers and bus conditions, thereby inciting a higher satisfaction with driver courtesy and safety. 
Also, the riders who use transit the most will have a more realistic view of bus dependability and allow for more time to make trip. A negative correlation between bus regularity and auto ownership suggests that since non-owners use transit often, they may have a more realistic understanding of arrival/departure time frames. Therefore, it is apparent that as ownership increases, satisfaction with bus regularity decreases.

Automobile owners may have extremely high expectations of transit service. For instance, owners tend to be less satisfied with the bus driver's ability to drive. A likely explanation is that automobile owners are used to driving and controlling a vehicle's whereabouts. Therefore, owners feel out of control when their rides depend on a complete stranger's driving abilities. Finally, lower income passengers perceived bus cleanliness and comfort to be more satisfactory than higher income passengers. This suggests that the riders who own automobiles are used to choosing their passengers. Auto owners may not be as comfortable with their fellow transit clientele and may be especially uncomfortable on crowded buses. 


\section{Ethnicity}

\begin{tabular}{|l|c|c|c|}
\hline & Table 17-I: PalmTran Ethnicity & White & Black \\
\hline Sample size & & $\mathbf{8 4 0}$ & $\mathbf{7 1 0}$ \\
\hline & & & \\
\hline SQ17 & Latest weekends & 2.85 & 3.29 \\
\hline SQ05 & Frequency of Service & 3.28 & 3.65 \\
\hline SQ15 & Latest weekdays & 2.97 & 3.32 \\
\hline SQ16 & Earliest weekends & 3.18 & 3.44 \\
\hline SQ07 & No. of transfers & 3.35 & 3.58 \\
\hline SQ08 & Ease of transfer & 3.46 & 3.68 \\
\hline SQ10 & Time to make trip & 3.42 & 3.64 \\
\hline SQ24 & Safety getting off bus & 3.84 & 4.05 \\
\hline
\end{tabular}

Blacks are more satisfied than whites on several specific elements of bus service, including span of service, transfers, and time to make trip. This may be due to the level of service provided to neighborhoods that are predominantly populated by bus users of different ethnic origins. If neighborhoods with more blacks tend to use bus service more than neighborhoods that are predominantly white, it is likely that the bus service to those neighborhoods is more frequent and runs later and would be more likely to go directly to desired destinations (or require one transfer), and hence ratings would be higher.

\begin{tabular}{|c|c|c|c|}
\hline & Table 17-II: Palm Tran Ethnicity & White & Hispanic \\
\hline Sample size & & $\mathbf{8 4 0}$ & $\mathbf{2 2 8}$ \\
\hline & & & \\
\hline SQ05 & Frequency of Service & 3.28 & 3.56 \\
\hline SQ08 & Ease of transfer & 3.46 & 3.73 \\
\hline SQ10 & Time to make trip & 3.42 & 3.65 \\
\hline
\end{tabular}

\begin{tabular}{|c|c|c|c|}
\hline & Table 17-III: PalmTran Ethnicity & Black & Hispanic \\
\hline Sample size & & $\mathbf{7 1 0}$ & $\mathbf{2 2 8}$ \\
\hline & & & \\
\hline SQ16 & Earliest weekends & 3.44 & 3.18 \\
\hline SQ15 & Latest weekdays & 3.32 & 2.97 \\
\hline SQ17 & Latest weekends & 3.29 & 2.91 \\
\hline
\end{tabular}


These findings appear to be a function of the type of service that is available to predominantly Hispanic neighborhoods. Apparently, service to those areas provides more direct and frequent service to desired destinations than service in white neighborhoods. However, service span is apparently more limited than service to predominantly black neighborhoods.

\begin{tabular}{|l|c|c|c|}
\hline & Table 17-IV: PalmTran Ethnicity & White & Other \\
\hline Sample size & & $\mathbf{8 4 0}$ & $\mathbf{1 1 7}$ \\
\hline & & & 3.64 \\
\hline SQ13 & Ease to use schedule & 3.83 & 2.77 \\
\hline SQ15 & Latest weekdays & 2.97 & 3.43 \\
\hline SQ14 & Earliest weekdays & 3.63 & 4.07 \\
\hline SQ28 & Ability to drive & 4.28 & 3.69 \\
\hline SQ26 & Temperature in bus & 3.93 & 3.64 \\
\hline SQ32 & Convenience of routes (combined SQ 6 \& 18) & 3.88 & 3.69 \\
\hline SQ27 & Availability of seats & 3.94 & 3.10 \\
\hline SQ10 & Time to make trip & 3.42 & 3.61 \\
\hline SQ11 & Value of fare/cost & 4.11 & \\
\hline
\end{tabular}

\begin{tabular}{|c|c|c|c|}
\hline & Table 17-V: PalmTran Ethnicity & Black & Other \\
\hline Sample size & & $\mathbf{7 1 0}$ & $\mathbf{1 1 7}$ \\
\hline & & & \\
\hline SQ07 & No. of transfers & 3.58 & 3.38 \\
\hline SQ31 & Satisfaction (combined) & 4.03 & 3.82 \\
\hline SQ14 & Earliest weekdays & 3.64 & 3.43 \\
\hline SQ08 & Ease of transfer & 3.68 & 3.46 \\
\hline SQ27 & Availability of seats & 3.93 & 3.69 \\
\hline SQ13 & Ease to use schedule & 3.93 & 3.64 \\
\hline SQ26 & Temperature in bus & 4.01 & 3.69 \\
\hline SQ17 & Latest weekends & 3.29 & 2.90 \\
\hline SQ05 & Frequency of Service & 3.65 & 3.26 \\
\hline SQ32 & Convenience of routes (combined SQ 6 \& 18) & 4.03 & 3.64 \\
\hline SQ16 & Earliest weekends & 3.44 & 3.04 \\
\hline SQ11 & Value of fare/cost & 4.03 & 3.61 \\
\hline SQ10 & Time to make trip & 3.64 & 3.10 \\
\hline SQ15 & Latest weekdays & 3.32 & 2.77 \\
\hline
\end{tabular}




\begin{tabular}{|l|c|c|c|}
\hline & Table 17-VI: PalmTran Ethnicity & Hispanic & Other \\
\hline Sample size & & $\mathbf{2 2 8}$ & $\mathbf{1 1 7}$ \\
\hline & & & 2.77 \\
\hline SQ15 & Latest weekdays & 2.97 & 3.93 \\
\hline SQ23 & Safety on bus & 4.13 & 3.43 \\
\hline SQ14 & Earliest weekdays & 3.64 & 4.07 \\
\hline SQ28 & Ability to drive & 3.30 & 3.46 \\
\hline SQ08 & Ease of transfer & 3.90 & 3.61 \\
\hline SQ12 & Ease to obtain schedule & 3.56 & 3.26 \\
\hline SQ05 & Frequency of Service & 4.13 & 3.82 \\
\hline SQ31 & Satisfaction (combined) & 3.96 & 3.64 \\
\hline SQ13 & Ease to use schedule & 3.97 & 3.64 \\
\hline SQ32 & Convenience of routes (combined SQ 6 \& 18) & 4.06 & 3.69 \\
\hline SQ26 & Temperature in bus & 4.07 & 3.69 \\
\hline SQ27 & Availability of seats & 4.07 & 3.61 \\
\hline SQ11 & Value of fare/cost & 3.65 & 3.10 \\
\hline SQ10 & Time to make trip & & \\
\hline
\end{tabular}

Members of 'Other' races, probably mainly Asians and native Americans, are significantly less satisfied with almost all elements of bus service than whites, Hispanics, and blacks. This may be partially a cultural phenomenon (i.e., a tendency to give lower ratings on surveys) but may also reflect lower level of service (i.e., less frequency, and later start / earlier end times) to neighborhoods predominantly populated by these racial groups. 
Gender

\begin{tabular}{|c|c|c|c|}
\hline & Table 18: PalmTran Gender & Male & Female \\
\hline Sample size & & $\mathbf{8 3 2}$ & $\mathbf{9 8 0}$ \\
\hline & & & \\
\hline SQ22 & Safety at bus stop & 3.93 & 3.68 \\
\hline
\end{tabular}

Generally differences between female and male ratings of satisfaction are not significantly different for Palm Beach transit riders. The differences that are observed are on the issue of safety at bus stops. Lower perception of safety at bus stops is not surprising - it is perhaps surprising that more safety issues were not rated differently by men and women. As it is, careful review of bus stop placements may help to reduce perceptions of danger among women. There may also be bus design characteristics that could be modified to better suit women. Qualitative investigation of these issues may help identify specific changes that can be made. 


\section{$\underline{\text { Recommendations }}$}

From the customer satisfaction model, it is possible to construct an "importanceperformance" matrix that graphically illustrates current bus riders' perceptions of MCAT's operations.

\section{Importance / Performance Matrix Palm Beach County Riders' perceptions of PalmTran service}

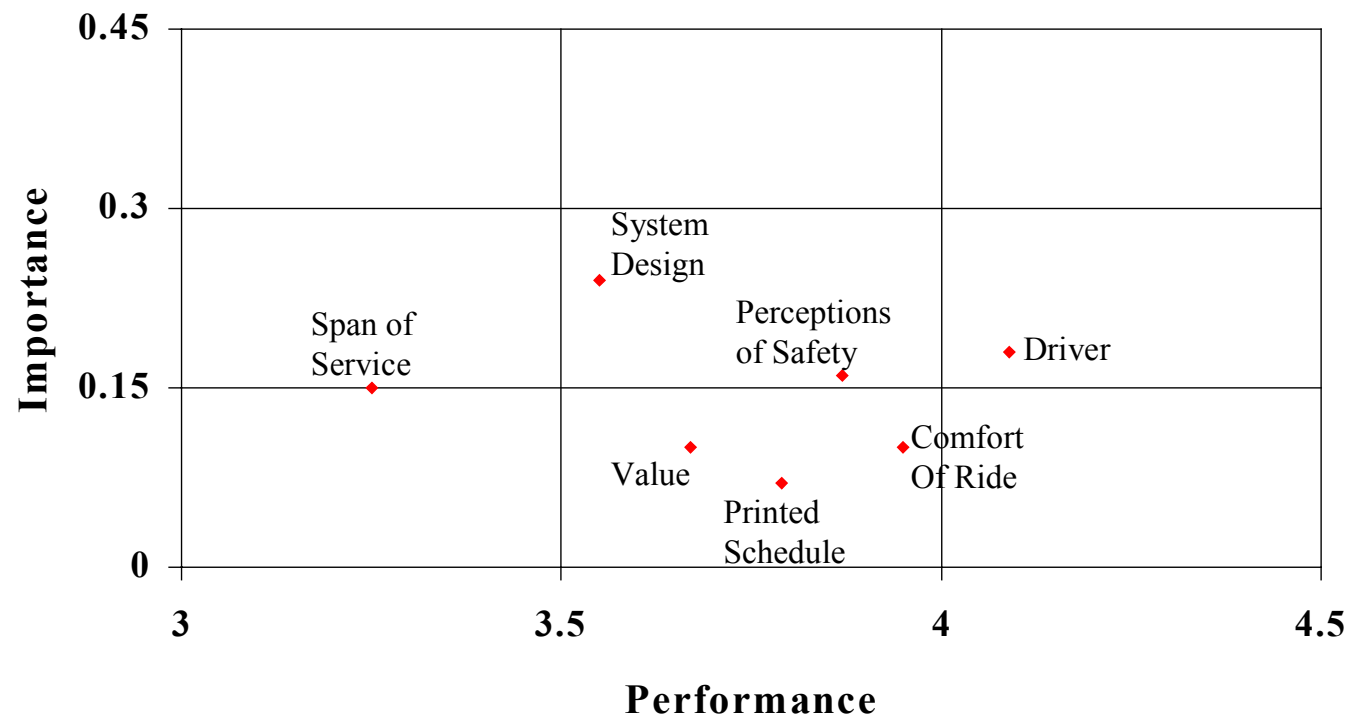

Figure 39: PalmTran Importance/Performance Matrix

The chart has been divided into nine regions, reflecting various combinations of low, medium, and high performance and low, medium, and high importance. Borderline figures are interpreted as being in the higher of the importance categories they border on, but the lower of the performance categories. This provides the most conservative interpretation of the results. The interpretations of the chart regions are done as follows: 


\begin{tabular}{|c|c|c|c|}
\hline \multicolumn{4}{|c|}{$\begin{array}{c}\text { Table } 19 \\
\text { Interpretations of PalmTran's Chart Regions }\end{array}$} \\
\hline \multicolumn{2}{|c|}{ Chart region } & \multirow[b]{2}{*}{ Interpretation } & \multirow[b]{2}{*}{ Areas } \\
\hline Importance & Performance & & \\
\hline Low & High & Possibly reduce focus on this area & \\
\hline Low & Medium & Maintain performance - no action & $\begin{array}{l}\text { Value, Printed Schedule, } \\
\text { Comfort of Ride }\end{array}$ \\
\hline Low & Low & Maintain performance - no action & \\
\hline Medium & High & Maintain performance - no action & Driver \\
\hline Medium & Medium & Maintain performance - no action & $\begin{array}{l}\text { System Design, } \\
\text { Perceptions of Safety }\end{array}$ \\
\hline Medium & Low & Investigate for improvements & Span of Service \\
\hline High & High & $\begin{array}{l}\text { Maintain performance - vigorous } \\
\text { quality checks, constant attention }\end{array}$ & \\
\hline High & Medium & Investigate for improvements & \\
\hline High & Low & Critical improvement area & \\
\hline
\end{tabular}

Span of Service is the only factor in the model that falls into the category of 'Investigate for improvements' on the chart. System Design issues should also be considered, as that factor falls into an area that closely borders on an 'investigate for improvements' section. 
The individual Span of Service items that PalmTran scores particularly low on are:

\begin{tabular}{|ll|c|}
\hline \multirow{2}{*}{\multicolumn{1}{c}{ Item }} & \multicolumn{2}{c|}{ Scores } \\
\cline { 2 - 3 } \multicolumn{1}{c|}{} & Index & Mean \\
\hline Latest weekdays & 99.03 & 3.07 \\
\hline Latest weekends & 97.25 & 3.02 \\
\hline Earliest weekends & 94.99 & 3.28 \\
\hline
\end{tabular}

The individual System Design items that PalmTran scores relatively low on are:

\begin{tabular}{|ll|c|}
\hline \multirow{2}{*}{\multicolumn{1}{c}{ Item }} & \multicolumn{2}{c|}{ Scores } \\
\cline { 2 - 3 } \multicolumn{1}{c|}{} & Index & Mean \\
\hline Number of Transfers & 95.28 & 3.45 \\
\hline Ease of Transfers & 91.26 & 3.56 \\
\hline Frequency of Service & 99.62 & 3.45 \\
\hline
\end{tabular}

The above table and Table III-B indicate that PalmTran's clients are usually more satisfied with System Design than the other transit agencies' customers. In fact, "ability to get where you want to go," "how regularly buses arrive on time," and "time to make trip" were rated higher than the state average. "Frequency of service" was regarded at an average level compared to other agencies in the state. The only area that may need improvement pertains to transfers. Both "number of transfers" and "ease of transfers" seem to have scored lower compared to the other items. Therefore, resources could be utilized to increase the satisfaction of these categories (e.g., alleviate the number of transfers needed to cross town; reconsider transfer ticket policies).

Span of Service issues are always rated at a low absolute level, almost regardless of which system is being examined. However, the above table indicates that even PalmTran's index scores are low - particularly on weekend service. PalmTran should consider increasing service at each end of the weekend service day. 
According to the individual index and mean scores PalmTran is doing a good job with respect to the Driver factor category. The overall mean indicates that this category is one of PalmTran's strengths. Moreover, the passengers' perception of "ability to drive," "driver courtesy," and "ease of using schedule" were high. Therefore, PalmTran should continue to support its drivers and provide its exemplary service in this category.

Table III-E reveals that PalmTran's customers are satisfied with the Perceptions of Safety category, too. The overall Safety mean was high, albeit lower than the Driver factor category. Also, compared to the other systems in Florida, PalmTran's passengers are fairly satisfied with the items contained within the Safety category: "safety on bus stop," safety on bus," "safety getting off bus," "clean buses and stop." Again, no major alterations are needed to increase standards in this category.

Demographic analysis indicates mainly structural factors such as level of choice ridership and familiarity with the bus system that impact satisfaction. However, the analysis did not identify specific, system-related recommendations for improvements. Rather, explanations related to choice ridership and familiarity seem to explain most of the variations in satisfaction. Service does appear to be better into neighborhoods that are predominantly black. Women rated safety at bus stops lower than men. A careful analysis of bus stop placements may help to reduce feelings of insecurity at bus stops among women. 


\section{RTS}

\section{$\underline{\text { Factor Analysis }}$}

Factor 2 - $\quad$ Span of Service

Major loadings - $\quad$ SQ17 Latest weekends

SQ15 Latest weekdays

SQ16 Earliest weekends

This construct relates to customer satisfaction with span of transit service availability. The components are satisfaction with latest weekday, earliest weekend, and latest weekend service.

Earliest weekday service loads on to the "Value" construct, indicating that a major element of customer perception of value is time of earliest weekday service for either getting to work or to early classes.

\begin{tabular}{|c|c|c|}
\hline \multirow[b]{3}{*}{ Item } & an of $S$ & \\
\hline & \multicolumn{2}{|c|}{ Scores } \\
\hline & Index & Mean \\
\hline Latest weekdays & 88.47 & 2.75 \\
\hline Latest weekends & 94.21 & 2.92 \\
\hline Earliest weekends & 94.73 & 3.27 \\
\hline Overall Mean & & 2.98 \\
\hline
\end{tabular}


Factor 3 - $\quad$ Perceptions of Safety

Major loadings - $\quad$ SQ22 Safety at bus stop

SQ24 Safety getting off bus

SQ20 Clean buses and stop

SQ23 Safety on bus

Minor loadings - $\quad$ SQ28 Ability to drive

SQ26 Temperature in bus

This construct relates to customer satisfaction with safety at bus stop, safety getting off bus, clean buses and stops, safety on bus. The connection between safety and cleanliness requires some explanation. One possibility is that the passenger construct relates to the apparent level of concern and respect the transit agency has for their passengers.

Cleanliness is a very common way of showing respect and concern for customers - in restaurants, retail businesses, even in personal interactions. One of the correlates of transit agency concern for customers could be safety at bus stops, since the perception that the agency is taking care of its equipment and facilities may transfer over into perceptions of safety.

Ability to drive and temperature are also minor construct loadings. The ability to drive likely relates directly to passenger feelings of safety as the bus drives along its route. Its impact on this construct is minor. Temperature also has a minor impact, possibly reflecting either crowding conditions (although availability of seats did not load on to the construct) or an indirect connection to concern of the transit agency for its customers.

\begin{tabular}{|lc|c|}
\hline \multicolumn{2}{|c|}{ Table 20-II: RTS Factor 3 - Perceptions of Safety } \\
\cline { 2 - 3 } & \multicolumn{2}{c|}{ Scores } \\
\cline { 2 - 3 } Item & Index & Mean \\
\hline Safety at Bus Stop & 99.41 & 3.89 \\
\hline Safety Getting off Bus & 98.75 & 4.01 \\
\hline Clean Buses and Stop & 98.86 & 3.83 \\
\hline Safety on Bus & 98.46 & 4.08 \\
\hline Ability to Drive & 95.73 & 4.10 \\
\hline Temperature in Bus & 95.36 & 3.67 \\
\hline Overall Mean & & $\mathbf{3 . 9 3}$ \\
\hline
\end{tabular}


Factor 4 - $\quad \underline{\text { Transfers }}$

Major loadings - $\quad$ SQ7 Number of transfers

SQ8 Ease of transfers

SQ6 Ability to get where you want to go

This construct relates to customer satisfaction with the number of transfers, ease of transfers and ability to get where you want to. Clearly the number and ease of making transfers has a major impact on customer perception of being able to get where they want to go.

\begin{tabular}{|lc|c|}
\hline \multicolumn{3}{|c|}{ Table 20-III: RTS Factor 4- Transfers } \\
\cline { 2 - 3 } & \multicolumn{2}{c|}{ Scores } \\
\cline { 2 - 3 } Item & Index & Mean \\
\hline Number of Transfers & 101.56 & 3.68 \\
\hline Ease of Transfers & 88.01 & 3.43 \\
\hline Ability to Get Where You Want to Go & 95.31 & 3.73 \\
\hline Overall Mean & 3.61 \\
\hline
\end{tabular}


Factor 5 - Value

Major loadings - $\quad$ SQ14 Earliest weekdays

SQ11 Value of fare/cost

Minor loadings - $\quad$ SQ16 Earliest weekends

SQ10 Time to make trip

We interpret this construct as being strongly related to customer satisfaction with the value of the fare. It is quite evident that early morning service is strongly tied to this perception, as shown by the strong loading of earliest weekday service and minor loading of earliest weekend service.

Time to make trip indicates that to some extent, the time involved in the trip may another issue in value perception.

\begin{tabular}{|lc|c|}
\hline \multicolumn{3}{|c|}{ Table 20-IV: RTS Factor 5 - Value } \\
\cline { 2 - 3 } & \multicolumn{2}{c|}{ Scores } \\
\cline { 2 - 3 } Item & Index & Mean \\
\hline Earliest Weekdays & 101.93 & 3.82 \\
\hline Value of Fare/Cost & 89.71 & 3.68 \\
\hline Earliest Weekends & 94.73 & 3.27 \\
\hline Time to Make Trip & 89.22 & 3.32 \\
\hline Overall Mean & 3.52 \\
\hline
\end{tabular}


Factor 6 - $\quad$ Comfort of Ride

Major loadings - SQ27 Availability of seats

SQ26 Temperature on bus

Minor loading - $\quad$ SQ28 Ability to drive

This construct is focused entirely on physical ride comfort, being comprised of the variables availability of seats and temperature in the bus, as well as the actual experience of the ride itself, as evidenced by the rating of the driver's ability to drive the bus.

\begin{tabular}{|lc|c|}
\hline \multicolumn{3}{|c|}{ Table 20-V: RTS Factor 6 - Comfort of Ride } \\
\hline \multicolumn{2}{|c|}{ Scores } \\
\cline { 2 - 3 } \multicolumn{1}{|c|}{ Item } & Index & Mean \\
\hline Availability of Seats & 89.18 & 3.48 \\
\hline Temperature on Bus & 95.36 & 3.67 \\
\hline Ability to Drive & 95.73 & 4.10 \\
\hline Overall Mean & & 3.75 \\
\hline
\end{tabular}


Factor 7 - $\quad \underline{\text { Schedule }}$

Major loadings - $\quad$ SQ12 Ease to obtain schedule

SQ13 Ease to use schedule

The construct relates to customer experience using schedules. The main variables in this construct are satisfaction with the ease of using the schedule and ease of obtaining schedule.

\begin{tabular}{|c|c|c|}
\hline \multicolumn{3}{|c|}{ Table 20-VI: RTS Factor 7 - Schedule } \\
\hline \multirow[b]{2}{*}{ Item } & \multicolumn{2}{|c|}{ Scores } \\
\hline & Index & Mean \\
\hline Ease to Obtain Schedule & 90.68 & 3.71 \\
\hline Ease to Use Schedule & 92.99 & 3.82 \\
\hline Overall Mean & & 3.77 \\
\hline
\end{tabular}


Factor 10 - Timeliness of Service

Major loadings - $\quad$ SQ5 Frequency of Service

SQ9 How regularly buses arrive on time

SQ10 Time to make trip

Minor loadings - $\quad$ SQ11 Value of fare/cost

SQ6 Ability to get where you want to go

This construct relates to issues of buses running frequently and on time, and the time to make trip. Also, convenience of routes slightly loaded on to this factor. All of these elements have an impact on customer value perception as well. These variables are connected with the customer's ability to arrive at their destination in a timely manner.

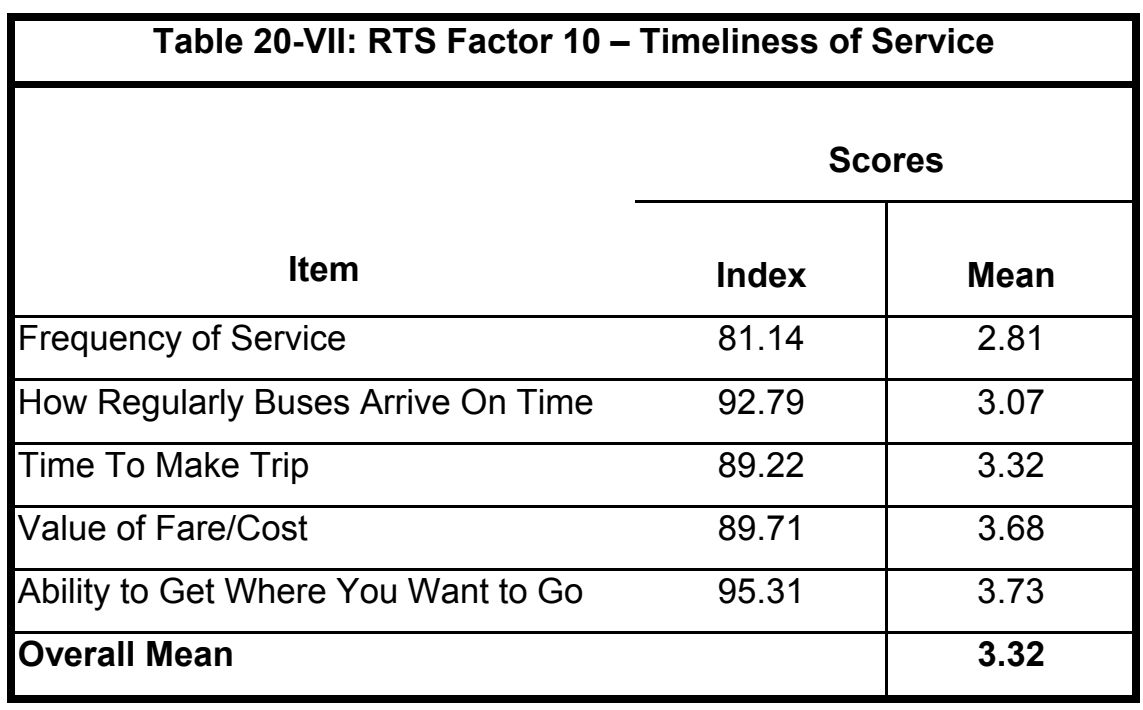


Factor 11 - Driver

Major loadings - $\quad$ SQ29 Driver courtesy

SQ28 Ability to drive

Minor loading - $\quad$ SQ23 Safety on bus

This construct relates to customer satisfaction with bus driver performance. The two main variables are driver courtesy and ability to drive the bus.

The safety rating may be included in this construct because high levels of driver courtesy could lead customers to believe that the driver, as the representative of the system, cares about them and will watch for their physical well being i.e. safety.

\begin{tabular}{|lc|c|}
\hline \multicolumn{3}{|c|}{ Table 20-VIII: RTS Factor 11 - Driver } \\
\hline \multirow{2}{*}{ Item } & \multicolumn{2}{c|}{ Scores } \\
\cline { 2 - 3 } & Index & Mean \\
\hline Driver Courtesy & 98.75 & 4.26 \\
\hline Ability to Drive & 95.73 & 4.10 \\
\hline Safety on Bus & 98.46 & 4.08 \\
\hline Overall Mean & & 4.15 \\
\hline
\end{tabular}




\section{$\underline{\text { Customer Satisfaction Model }}$}

The following chart indicates which of the RTS factors are the most important. The most influential factors are those with the largest importance values. For instance, since Transfers and Timeliness of Service have the highest importance values, RTS should devote the most time and energy to these categories in order to improve customer satisfaction ratings. By looking at the individual items in each of these constructs, management can determine which items to focus on.

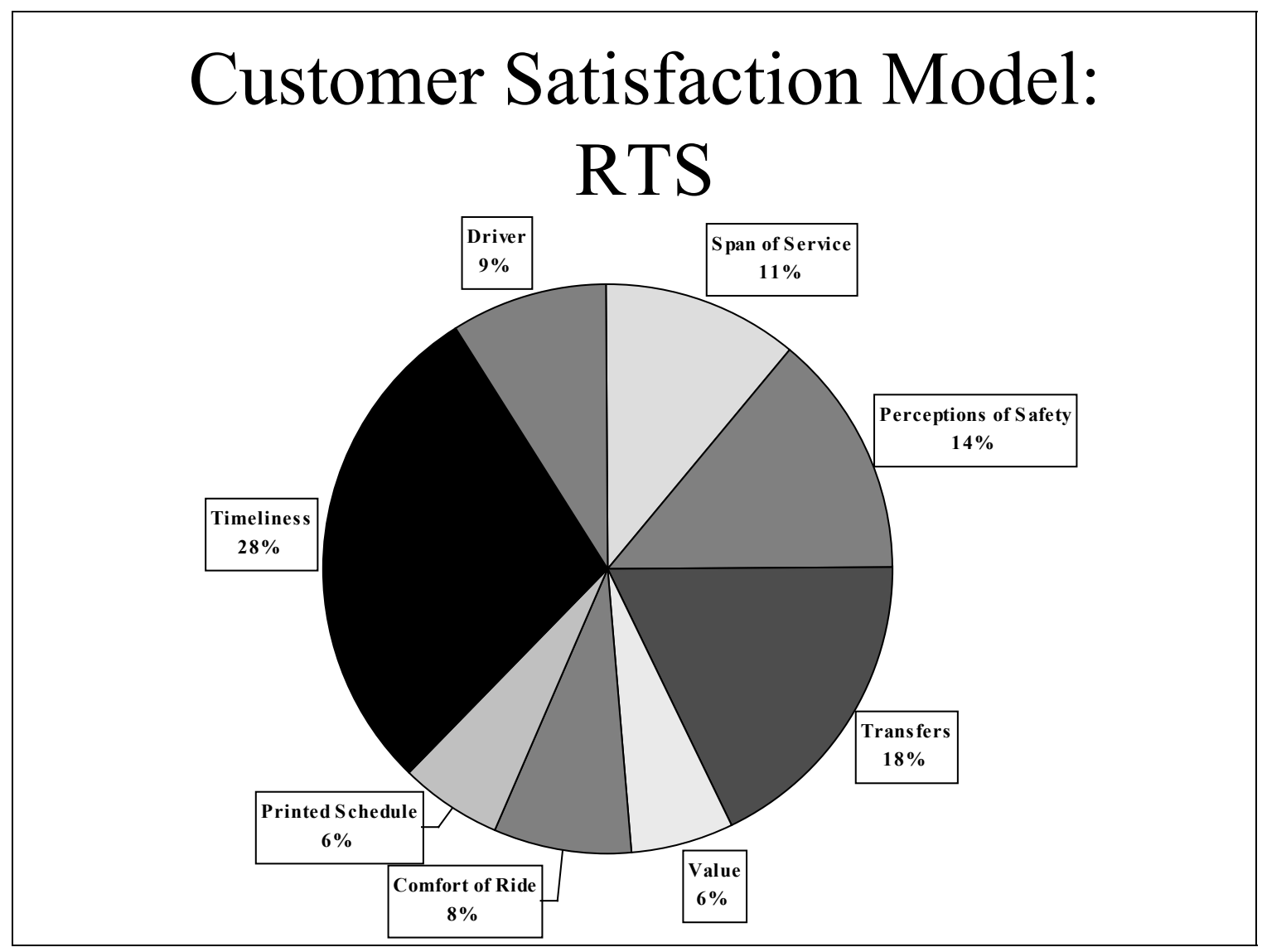

Figure 40: RTS Customer Satisfaction Model 
$\underline{\text { Demographic Analysis }}$

Income

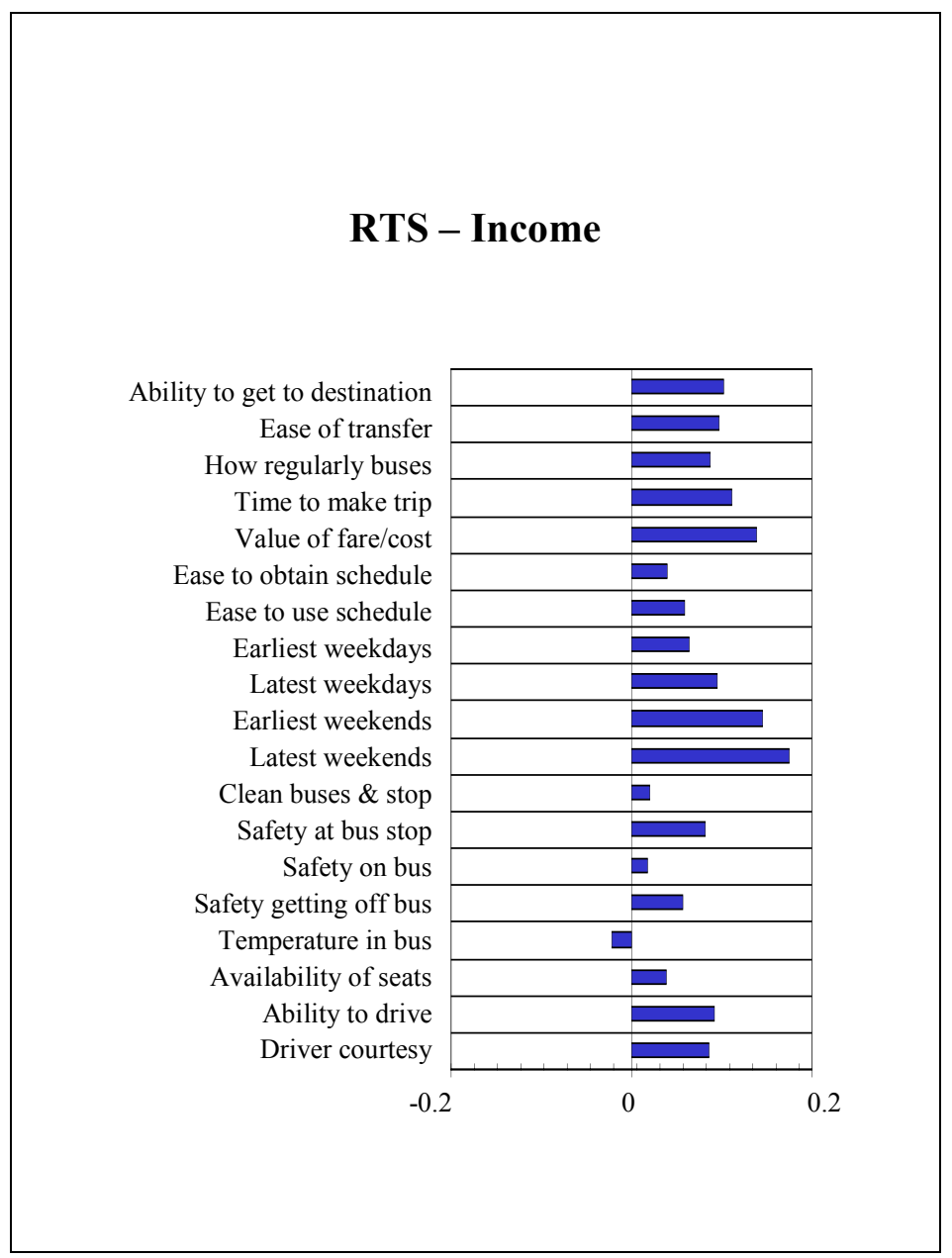

Figure 41: RTS Correlation of income with satisfaction items

An analysis of non-linearly related items reveals that satisfaction with frequency of service increases as income increases, and that satisfaction with number of transfers is lower for those with incomes of $\$ 15,000-\$ 25,000$ than for any other group.

RTS' higher income riders are more satisfied with transit service than lower income riders. This finding is likely due to the fact that higher income riders are more likely to be choice riders and hence will only use the bus when it is convenient for them. Thus those of higher income who are potential riders who feel that the bus is not convenient (in 
terms of routes, span of service, etc.) will choose not to ride the bus and would therefore not be sampled. Lower income riders do not have this choice.

Choice riders perceive transit service differently. Since choice riders utilize transit only when it is convenient, they are inclined to be more satisfied with measures such as ability to get where, bus regularity, and ease of transfer. Moreover, assuming higher income individuals have access to other forms of transportation, they tend to be more satisfied with weekday and weekend service start and end times. The higher income groups rate RTS' fare as being more valuable. This can be derived from a combination of circumstances (such as fare as a percentage of income and riding the bus only when it is convenient).

Higher income individuals perceived comfort factors to be more satisfactory. They rated seat availability, ability to drive, and driver courtesy higher than low-income riders. Again, this probably relates to the fact that higher income individuals tend to ride buses in the nicer neighborhoods. Firstly, less passenger crowding causes seat availability to be rated higher. Secondly, roads that are located within high-income neighborhoods tend to be maintained better. Therefore, the 'ability to drive' rating may be higher due to better maintenance (i.e. fewer potholes) of the roads used on these routes. Moreover, drivers may be more relaxed and friendly when encountering a well-maintained stretch of road.

High-income patrons are more satisfied with safety at the bus stop, safety on the bus, and safety getting off bus because due to their higher income status they live in lower crime areas and are less likely to travel on the bus at night. Low-income riders rely on the bus more and may live in higher crime areas and must travel on the bus regardless of the time of day. They are, therefore, likely to be less satisfied with their safety on the bus.

RTS encounters unique ridership patterns due to its proximity to the University of Florida. The University of Florida supplements RTS' fares through a contract that allows students and faculty members free fares near the campus. Lower fares combined with convenience lead to more frequent ridership. Due to the frequent ridership, college affiliated persons tend to rate ease to use schedule higher. Moreover, RTS' marketing efforts on campus have made route schedules readily available. Therefore, it is no surprise that a positive correlation between ease to obtain schedule and income exists. 
The only item with which lower income riders are more satisfied is temperature in bus. Lower income riders probably use their familiarity with transit to set more realistic expectations for these elements of the bus-riding than higher income riders. Thus the experiences that occur for which no prior expectation can reasonably be formed without prior use of the bus, such as temperature on the bus may be expected to get higher ratings among lower-income users. 


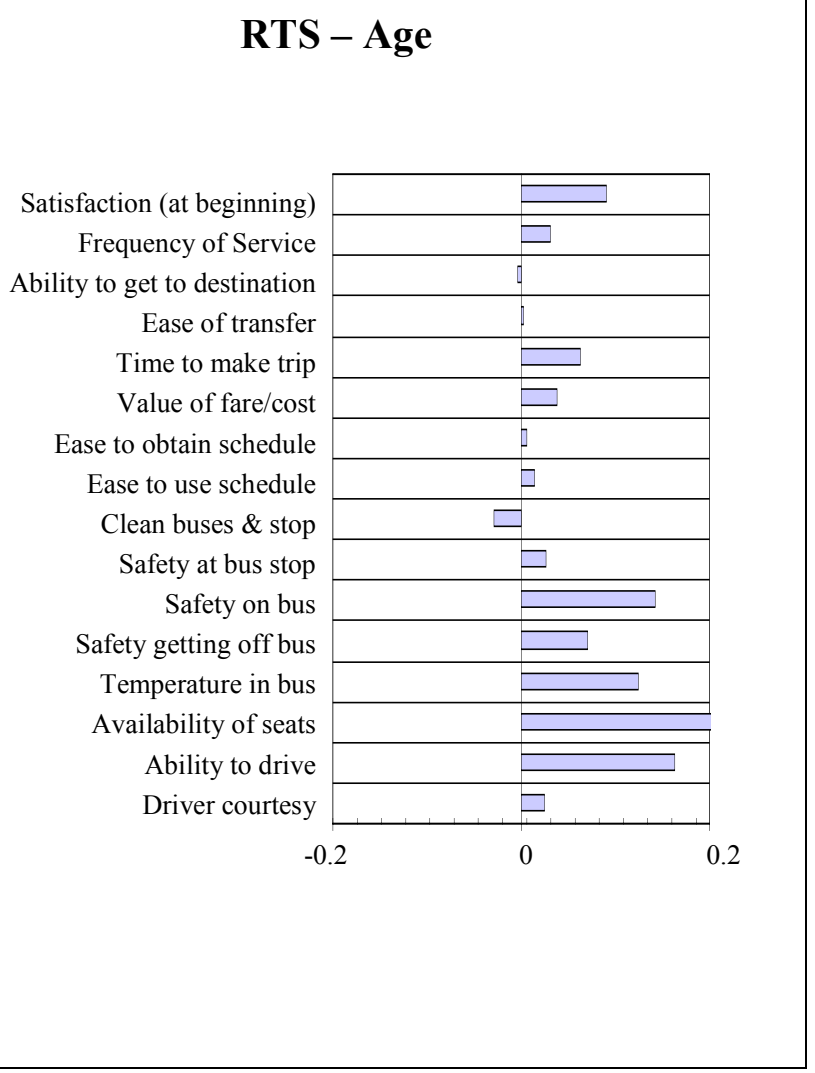

Figure 42: RTS Correlation of age with satisfaction items

An analysis of non-linearly related items indicates that satisfaction with number of transfers and with span of service is generally higher for younger passengers.

Almost all of the satisfaction factors are positively correlated with age. Older respondents are overwhelmingly satisfied with transit service. As age increases, satisfaction increases for all but three categories: ability to get where passengers want to go, span of service, and clean buses and stops. Different experiences between the older and younger individuals may cause the satisfaction differences between younger and older individuals. For example, individual time constraints and amount of leisure time can influence a passenger's satisfaction level. Since younger people are usually on a tighter schedule, such as arriving to work on time, they will usually be more conscience 
of time delays. This can lead younger people to be less satisfied with frequency of service, ease of transfer, and time to make trip.

A combination of familiarity with surroundings as well as non-peak trips can lead to higher satisfaction ratings. Elderly people tend to utilize transit services in their particular neighborhood. Therefore, they are usually more familiar with their surroundings. Those who are more familiar with their surroundings tend to be generally more comfortable. Familiarity can lead older people to bestow higher ratings to driver courtesy, ability to drive, ease to obtain schedule, ease to use schedule, and safety factors.

Finally, fare structure causes passengers to rate the value of transit differently. Due to RTS' unusual fare structure, one should hesitate before making broad assumptions. Senior citizens receive half off regular fare. Moreover, anyone affiliated with the University of Florida receives a free fare. Therefore, a positive correlation can not be explained via senior citizen fare reduction only. 


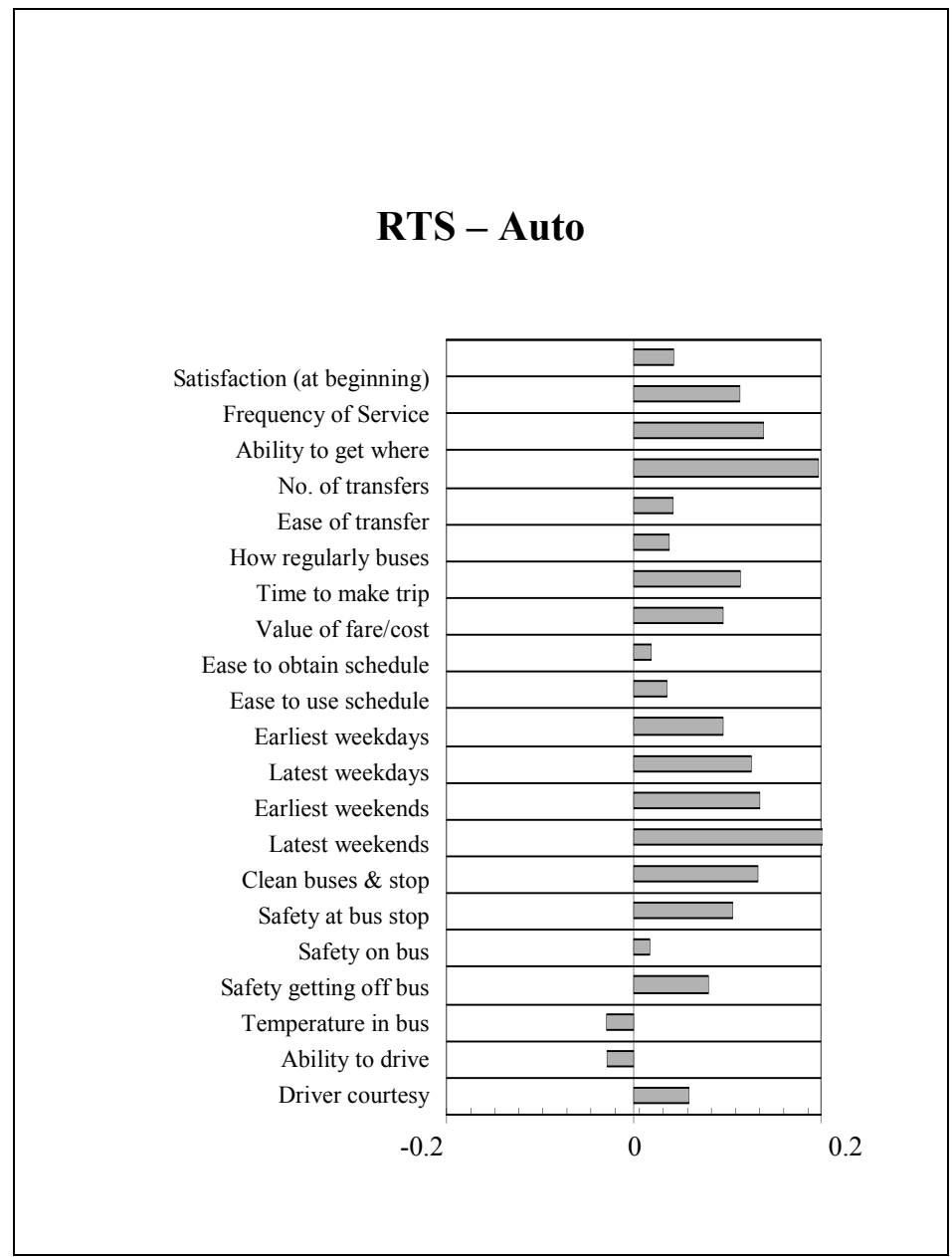

Figure 43: RTS Correlation of auto ownership with satisfaction items

An analysis of a non-linearly related item, satisfaction with auto ownership, indicates that those with lower levels of auto ownership are more satisfied with availability of seats.

Auto ownership impacts these satisfaction levels because there is a difference between riding buses due to convenience versus riding them out of necessity. Households that own automobiles have the luxury of 24-hour transportation. In contrast, households without vehicles are constrained by transit service times, taxi costs, and/or friends' schedules. Due to the fact that those who own automobiles have an alternate form of transportation at their disposal, it is not surprising to obtain many positive satisfaction correlations between auto ownership and transit service categories. In fact, the positive satisfaction correlations between auto ownership and service frequency, number of 
transfers, ease of transfer, time to make trip, bus regularity, and weekend/weekday service schedule suggest that auto owners ride the bus when it is a convenience.

As car ownership increases, satisfaction for number of transfers increases. This positive relationship reveals a car owner's tendency to utilize transit for short trips. In contrast, those without cars rely on transit for all destinations. Usually, longer trips require more transfers, which can be seen as cumbersome by those who use transit as their only reliable form of transportation.

Unlike the All Systems results, satisfaction of the value of fare/cost is positively correlated with number of automobiles in household. Value of fare/cost was perceived to be more satisfying by those with vehicles. Assuming passengers who own cars receive a larger income, the fare would be a smaller percentage of take home salary for them. Also, those with vehicles know the true cost of providing transportation and would thereby consider transit service a better buy than those without a vehicle.

Automobile owners may have extremely high expectations of transit service. For instance, owners tend to be less satisfied with the bus driver's ability to drive. A likely explanation is that automobile owners are used to driving and controlling a vehicle's whereabouts. Therefore, owners feel out of control when their rides depend on a complete stranger's driving abilities. Finally, lower income passengers perceived bus temperature to be more satisfactory than higher income passengers. This suggests that the riders who own automobiles are used to less passenger congestion. Auto owners may not be as comfortable with their fellow transit clientele and may be especially uncomfortable on crowded buses. 


\section{Ethnicity}

\begin{tabular}{|c|c|c|c|}
\hline & Table 21-I: RTS Ethnicity & White & Black \\
\hline Sample size & & $\mathbf{6 3 7}$ & $\mathbf{2 8 4}$ \\
\hline & & & \\
\hline SQ15 & Latest weekdays & 2.71 & 2.94 \\
\hline SQ14 & Earliest weekdays & 3.90 & 3.70 \\
\hline SQ22 & Safety at bus stop & 3.97 & 3.75 \\
\hline SQ10 & Time to make trip & 3.39 & 3.17 \\
\hline SQ29 & Driver courtesy & 4.36 & 4.12 \\
\hline SQ31 & Satisfaction (combined) & 3.73 & 3.43 \\
\hline SQ34 & Cleanliness/comfort (combined SQ 20 \& 21) & 3.95 & 3.58 \\
\hline SQ11 & Value of fare/cost & 3.81 & 3.34 \\
\hline SQ07 & No. of transfers & 3.90 & 3.21 \\
\hline
\end{tabular}

In most cases, whites have higher levels of satisfaction than blacks. This is probably related to many of the same factors that create higher satisfaction levels among higher income riders - mainly because they can be choice riders and are more apt to use the transit when it is convenient, but always having the option to drive where transit service does not meet their need for convenience and speed.

On only one item did blacks have higher ratings than whites. That item was 'Satisfaction with latest weekday service,' which for both groups and in total was the lowest rated item in the survey. This is somewhat surprising, since it is expected that whites are more likely to be choice riders and presumably would not be as affected by service end times. The finding may either be a random aberration or it may be that the earlier end times for service in areas predominantly populated by whites affects white riders so much that the impact of having more choice rider in that population segment is overwhelmed. 


\begin{tabular}{|c|c|c|c|}
\hline & Table 21-II: RTS Ethnicity & White & Hispanic \\
\hline Sample size & & $\mathbf{6 3 7}$ & $\mathbf{9 1}$ \\
\hline & & & \\
\hline SQ05 & Frequency of Service & 2.84 & 3.10 \\
\hline SQ26 & Temperature in bus & 3.69 & 3.28 \\
\hline
\end{tabular}

Whites and Hispanics have largely equal levels of satisfaction. Hispanics are slightly more satisfied with Frequency of Service, Whites more satisfied with temperature in the buses. This may be either due to overcrowding on routes used by Hispanics. It's also possible that Hispanics feel the buses are too cold - perhaps they would prefer a higher AC setting on the buses than whites.

\begin{tabular}{|c|c|c|c|}
\hline & Table 21-III: RTS Ethnicity & Black & Hispanic \\
\hline Sample size & & $\mathbf{2 8 4}$ & $\mathbf{9 1}$ \\
\hline & & & \\
\hline SQ11 & Value of fare/cost & 3.34 & 3.96 \\
\hline SQ07 & No. of transfers & 3.21 & 3.83 \\
\hline SQ05 & Frequency of Service & 2.72 & 3.10 \\
\hline SQ31 & Satisfaction (combined) & 3.43 & 3.70 \\
\hline SQ32 & Convenience of routes (combined SQ 6 \& 18) & 3.68 & 3.89 \\
\hline SQ34 & Cleanliness/comfort (combined SQ 20 \& 21) & 3.58 & 3.77 \\
\hline SQ15 & Latest weekdays & 2.94 & 2.66 \\
\hline SQ26 & Temperature in bus & 3.73 & 3.28 \\
\hline
\end{tabular}

Hispanics are generally more satisfied with bus service than blacks. The elements where Hispanics are less satisfied are latest weekday service and temperature in the bus. Again, it appears that bus service into predominantly black neighborhoods runs later than bus service elsewhere. It should be noted that even the more satisfied group, the blacks, are not particularly highly satisfied (only a 2.94 on a 1-5 scale). 


\begin{tabular}{|c|c|c|c|}
\hline & Table 21-IV: RTS Ethnicity & White & Other \\
\hline Sample size & & $\mathbf{6 3 7}$ & $\mathbf{1 6 9}$ \\
\hline & & & \\
\hline SQ12 & Ease to obtain schedule & 3.75 & 3.52 \\
\hline SQ29 & Driver courtesy & 4.36 & 4.11 \\
\hline SQ07 & No. of transfers & 3.90 & 3.61 \\
\hline SQ08 & Ease of transfer & 3.49 & 3.19 \\
\hline SQ32 & Convenience of routes (combined SQ 6 \& 18) & 3.79 & 3.47 \\
\hline
\end{tabular}

\begin{tabular}{|c|c|c|c|}
\hline & Table 21-V: RTS Ethnicity & Black & Other \\
\hline Sample size & & $\mathbf{2 8 4}$ & $\mathbf{1 6 9}$ \\
\hline & & & \\
\hline SQ07 & No. of transfers & 3.21 & 3.61 \\
\hline SQ11 & Value of fare/cost & 3.34 & 3.67 \\
\hline SQ34 & Cleanliness/comfort (combined SQ 20 \& 21) & 3.58 & 3.84 \\
\hline SQ10 & Time to make trip & 3.17 & 3.38 \\
\hline SQ33 & Dependability of buses (on time) (combined SQ 9 \& 19) & 3.18 & 2.98 \\
\hline SQ32 & Convenience of routes (combined SQ 6 \& 18) & 3.68 & 3.47 \\
\hline SQ12 & Ease to obtain schedule & 3.75 & 3.52 \\
\hline SQ08 & Ease of transfer & 3.47 & 3.19 \\
\hline SQ15 & Latest weekdays & 2.94 & 2.65 \\
\hline
\end{tabular}

\begin{tabular}{|c|c|c|c|}
\hline & Table 21-VI: RTS Ethnicity & Hispanic & Other \\
\hline Sample size & & $\mathbf{9 1}$ & $\mathbf{1 6 9}$ \\
\hline & & & \\
\hline SQ26 & Temperature in bus & 3.28 & 3.67 \\
\hline SQ33 & Dependability of buses (on time) (combined SQ 9 \& 19) & 3.18 & 2.98 \\
\hline SQ07 & No. of transfers & 3.83 & 3.61 \\
\hline SQ12 & Ease to obtain schedule & 3.75 & 3.52 \\
\hline SQ08 & Ease of transfer & 3.47 & 3.19 \\
\hline SQ11 & Value of fare/cost & 3.96 & 3.67 \\
\hline SQ05 & Frequency of Service & 3.10 & 2.70 \\
\hline SQ32 & Convenience of routes (combined SQ 6 \& 18) & 3.89 & 3.47 \\
\hline
\end{tabular}

Members of 'Other' races, probably mainly Asians and native Americans, are significantly less satisfied with almost all elements of bus service than whites, Hispanics, and blacks. This may be partially a cultural phenomenon (i.e., a tendency to give lower 
ratings on surveys) but may also reflect lower level of service (i.e., less frequency, and later start / earlier end times) to neighborhoods predominantly populated by these racial groups.

There are a few individual elements where members of 'Other' races are more satisfied value, number of transfers, and time to make trip versus blacks, and temperature in bus versus Hispanics. Black riders of the RTS systems consistently provide the lowest ratings for value, number of transfers, and time to make trips. O/D patterns for the black population may need to be re-established and routings revised to address their needs.

\section{Gender}

\begin{tabular}{|c|c|c|c|}
\hline & Table 22: RTS Gender & Male & Female \\
\hline Sample size & & $\mathbf{5 2 8}$ & $\mathbf{6 5 2}$ \\
\hline & & & \\
\hline SQ05 & Frequency of Service & 2.91 & 2.69 \\
\hline SQ07 & No. of transfers & 3.79 & 3.56 \\
\hline SQ27 & Availability of seats & 3.59 & 3.34 \\
\hline SQ10 & Time to make trip & 3.48 & 3.19 \\
\hline
\end{tabular}

For most items, males and females rated their satisfaction relatively equally. Men are more satisfied than women with frequency of service, number of transfers, availability of sears, and time to make trips. This may be due to men being more likely to be choice riders than women, due to wage differences and other factors. If women are more dependent on the bus for all their transportation needs, it would be logical if they were less satisfied with frequency of service, time to make trip, and number of transfers. Also, if they are using trips for non-work related purposes (such as shopping) it would be understandable if they were more acutely affected by availability of seats on buses. 


\section{$\underline{\text { Recommendations }}$}

From the customer satisfaction model, it is possible to construct an "importanceperformance" matrix that graphically illustrates current bus riders' perceptions of RTS operations.

\section{Importance / Performance Matrix} Gainesville Riders' perceptions of RTS service

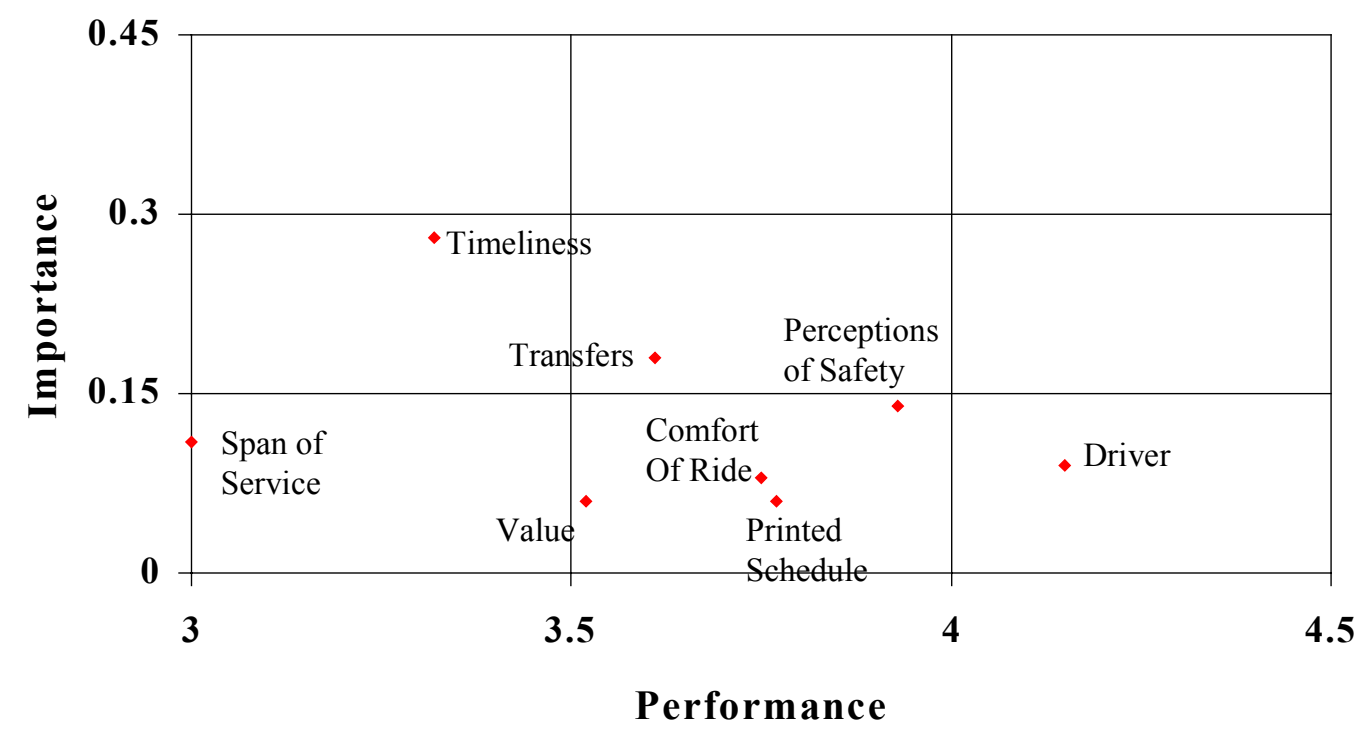

Figure 44: RTS Importance/Performance matrix

The chart has been divided into nine regions, reflecting various combinations of low, medium, and high performance and low, medium, and high importance. Borderline figures are interpreted as being in the higher of the importance categories they border on, but the lower of the performance categories. This provides the most conservative interpretation of the results. The interpretations of the chart regions are done as follows: 


\begin{tabular}{|c|c|c|c|}
\hline \multicolumn{4}{|c|}{$\begin{array}{c}\text { Table } 23 \\
\text { Interpretations of RTS Chart Regions }\end{array}$} \\
\hline \multicolumn{2}{|c|}{ Chart region } & \multirow[b]{2}{*}{ Interpretation } & \multirow[b]{2}{*}{ Areas } \\
\hline Importance & Performance & & \\
\hline Low & High & Possibly reduce focus on this area & Driver \\
\hline Low & Medium & Maintain performance - no action & $\begin{array}{l}\text { Value, Printed Schedule, } \\
\text { Comfort of Ride, } \\
\text { Perceptions of Safety }\end{array}$ \\
\hline Low & Low & Maintain performance - no action & Span of Service \\
\hline Medium & High & Maintain performance - no action & \\
\hline Medium & Medium & Maintain performance - no action & Transfers \\
\hline Medium & Low & Investigate for improvements & Timeliness \\
\hline High & High & $\begin{array}{l}\text { Maintain performance - vigorous } \\
\text { quality checks, constant attention }\end{array}$ & \\
\hline High & Medium & Investigate for improvements & \\
\hline High & Low & Critical improvement area & \\
\hline
\end{tabular}

Timeliness is the only factor in the model that falls into the category of 'Investigate for improvements' on the chart. Span of Service issues should also be considered since the satisfaction scores on that item are so low. 
The individual Span of Service items that RTS scores particularly low on are:

\begin{tabular}{|ll|c|}
\hline \multirow{2}{*}{\multicolumn{1}{c}{ Item }} & \multicolumn{2}{c|}{ Scores } \\
\cline { 2 - 3 } \multicolumn{1}{c|}{} & Index & Mean \\
\hline Latest weekdays & 88.47 & 2.75 \\
\hline Latest weekends & 94.21 & 2.92 \\
\hline Earliest weekends & 94.73 & 3.27 \\
\hline
\end{tabular}

The Timeliness items that RTS scores relatively low on are:

\begin{tabular}{|ll|c|}
\hline \multirow{2}{*}{\multicolumn{1}{c}{ Item }} & \multicolumn{2}{c|}{ Scores } \\
\cline { 2 - 3 } \multicolumn{1}{c|}{} & Index & Mean \\
\hline Frequency of Service & 81.14 & 2.81 \\
\hline How Regularly Buses Arrive On Time & 92.79 & 3.07 \\
\hline Time To Make Trip & 89.22 & 3.32 \\
\hline
\end{tabular}

Timeliness of Service indicates that some areas can be improved. In fact, this category showed the most impact on customer satisfaction, indicating that the greatest amount of resources should be devoted to the topics pertaining to this category. The "frequency of service" item contains disappointing statistics, indicating that this area should be a focal point when discussing improvements. "Time to make trip" can use improvements, too. Studies should be conducted to determine the best way to decrease the time it takes to make a trip. Also, there may be a need for priority busing strategies (i.e. HOV lanes; express routes). In contrast, passengers were happy with the "how regularly buses arrive on time" and "ability to get where you want to go" items located within the Timeliness category.

Similarly, Span of Service scores are quite low, particularly for late service. RTS should investigate $\mathrm{O} / \mathrm{D}$ patterns for trips that have demand for later service and investigate the feasibility of extending service hours for those routes. 
Table III-F reveals that RTS' customers are fairly satisfied with the Transfers category. According to the individual mean scores and comparative index scores, Gainesville's customers are primarily unsatisfied "ease of transfers." Its customers rated this item lower than the state average. Therefore, some resources should be dedicated to this area (i.e. reconsider transfer ticket policies). In contrast, "number of transfers" and "ability to get where you want to go" received high marks. Therefore, these items are satisfactory and the policies pertaining to these items should be continued.

Demographic analysis indicates mainly structural factors such as level of choice ridership and familiarity with the bus system that impact satisfaction. However, the analysis did not identify specific, system-related recommendations for improvements. Rather, explanations related to choice ridership and familiarity seem to explain most of the variations in satisfaction. Also, unique fare structures and marketing efforts related to service to the University of Florida create some atypical patterns of correlation, but do not yield specific improvement recommendations.

Whites generally have a higher level of satisfaction than blacks. This may be due to fare structures and UF service. However, RTS should assess service into core ethnic neighborhoods to ensure that level of service is at least as good in those areas as for areas that are less ethnically diverse and/or serve more affluent neighborhoods.

Women have significantly lower ratings on several items than men, including Frequency of service, number of transfers required, availability of seats, and time to make trip. This may indicate that trip purposes used by women may not be as well served as other trip purposes. Thus an investigation into these trip types and how to better service them may be in order. 


\section{SCAT - Sarasota}

$\underline{\text { Factor Analysis }}$

Factor 1 - $\quad$ System Design

Major loadings - $\quad$ SQ8 Ease of transfer

SQ18 Convenience of routes

Minor loadings - $\quad$ SQ5 Frequency of service

SQ10 Time to make trip

SQ19 Dependability of service (on time)

This construct includes customer satisfaction with ease of transfers, frequency of service and convenience of routes, on-time performance and time to make trip. The emphasis is on transfers, indicating that transfers are a major element in the overall satisfaction with the design of the system in filling customer travel needs.

\begin{tabular}{|lc|c|}
\hline \multicolumn{2}{|c|}{ Table 24-I: SCAT Factor 1 - System Design } \\
\cline { 2 - 3 } & \multicolumn{2}{c|}{ Scores } \\
\cline { 2 - 3 } Item & Index & Mean \\
\hline Ease of Transfer & 103.41 & 4.03 \\
\hline Convenience of Routes & 101.74 & 4.06 \\
\hline Frequency of Service & 109.80 & 3.80 \\
\hline Time to Make Trip & 107.84 & 4.01 \\
\hline Dependability of Service (On Time) & 105.10 & 4.10 \\
\hline Overall Mean & & $\mathbf{4 . 0 0}$ \\
\hline
\end{tabular}


Factor 2 - $\quad$ Span of Service

Major loadings - $\quad$ SQ4 Hours of service

SQ3 Days of service

SQ5 Frequency of service

The predominant variables in this construct are satisfaction with the hours and days of service. Frequency is a much less strong loading.

\begin{tabular}{|c|c|c|}
\hline \multirow[b]{2}{*}{ Item } & \multicolumn{2}{|c|}{ Scores } \\
\hline & Index & Mean \\
\hline Hours of Service & 99.21 & 3.60 \\
\hline Days of Service & 99.66 & 4.13 \\
\hline Frequency of Service & 109.80 & 3.80 \\
\hline Overall Mean & & 3.84 \\
\hline
\end{tabular}


Factor 7 - $\quad \underline{\text { Schedule }}$

Major loadings - $\quad$ SQ12 Ease to obtain schedule

SQ13 Ease to use schedule

Minor loading - $\quad$ SQ11 Value of fare/cost

The construct relates to customer experience using schedules. The main variables in this construct are satisfaction with the ease of using the schedule and ease of obtaining schedule. The loading of value onto this construct indicates that customers' perception of value is highly related to their ability to obtain and use schedules. As a result, it may be advantageous to make schedules more easily accessible to and interpretable by customers.

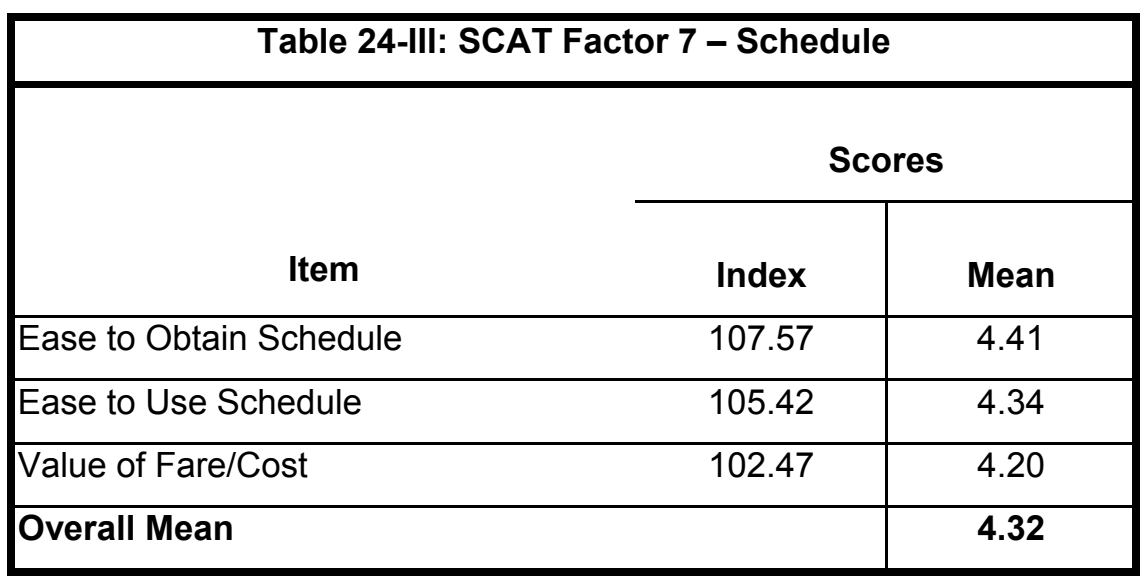


Factor 8 - $\quad \underline{\text { Value/Timeliness }}$

Major loadings - $\quad$ SQ19 Dependability of service (on time)

SQ11 Value of fare/cost

SQ10 Time to make trip

Minor loadings - $\quad$ SQ21 Cleanliness/comfort

SQ18 Convenience of routes

This construct relates to customer satisfaction with value of fare/cost, time to make trip, and on time-performance of buses. In addition, convenience of routes and cleanliness/comfort also loaded slightly on to this factor.

Time to make trip and buses running on time indicate that to a large extent, the time involved in the trip may be more of an issue than the cost of the fare.

\begin{tabular}{|lc|c|}
\hline \multicolumn{2}{|c|}{ Table 24-IV: SCAT Factor 8 - Value/Timeliness } \\
\cline { 2 - 3 } & \multicolumn{2}{c|}{ Scores } \\
\cline { 2 - 3 } Item & Index & Mean \\
\hline Dependability of Service & 105.10 & 4.10 \\
\hline Value of Fare/Cost & 102.47 & 4.20 \\
\hline Time to Make Trip & 107.84 & 4.01 \\
\hline Cleanliness/Comfort & 99.99 & 4.33 \\
\hline Convenience of Routes & 101.74 & 4.06 \\
\hline Overall Mean & $\mathbf{4 . 1 4}$ \\
\hline
\end{tabular}


Factor 9 - Experience of bus ride

Major loadings - $\quad$ SQ29 Driver courtesy

SQ25 Safety on bus and stops

SQ21 Cleanliness/comfort

Minor loading - $\quad$ SQ8 Ease of transfer

The construct encompasses all the major experiential elements involved in the bus ride perceptions of the bus stop (cleanliness and comfort), meeting the driver, cleanliness and comfort of the bus itself, and whatever perceptions of safety one might have while riding. The ease of transferring variable is probably a minor loading because not all customers transfer buses during their trips.

\begin{tabular}{|lc|c|}
\hline \multicolumn{2}{|c|}{ Table 24-V: SCAT Factor 9 - Experience of Bus Ride } \\
\hline \multirow{2}{*}{ Item } & Index & Mean \\
\cline { 2 - 3 } & 99.49 & 4.29 \\
\hline Driver Courtesy & 99.94 & 4.33 \\
\hline Safety on Bus and Stops & 99.99 & 4.33 \\
\hline Cleanliness/Comfort & 103.41 & 4.03 \\
\hline Ease of Transfer & & $\mathbf{4 . 2 5}$ \\
\hline Overall Mean & & \\
\hline
\end{tabular}




\section{$\underline{\text { Customer Satisfaction Model }}$}

The following chart indicates which of Sarasota's factors are the most important. The most influential factors are those with the largest importance values. For instance, since System Design and Span of Service have the highest importance values, SCAT should devote the most time and energy to these categories in order to improve customer satisfaction ratings. By looking at the individual items in each of these constructs, management can determine which items to focus on.

\section{Customer Satisfaction Model: SCAT}

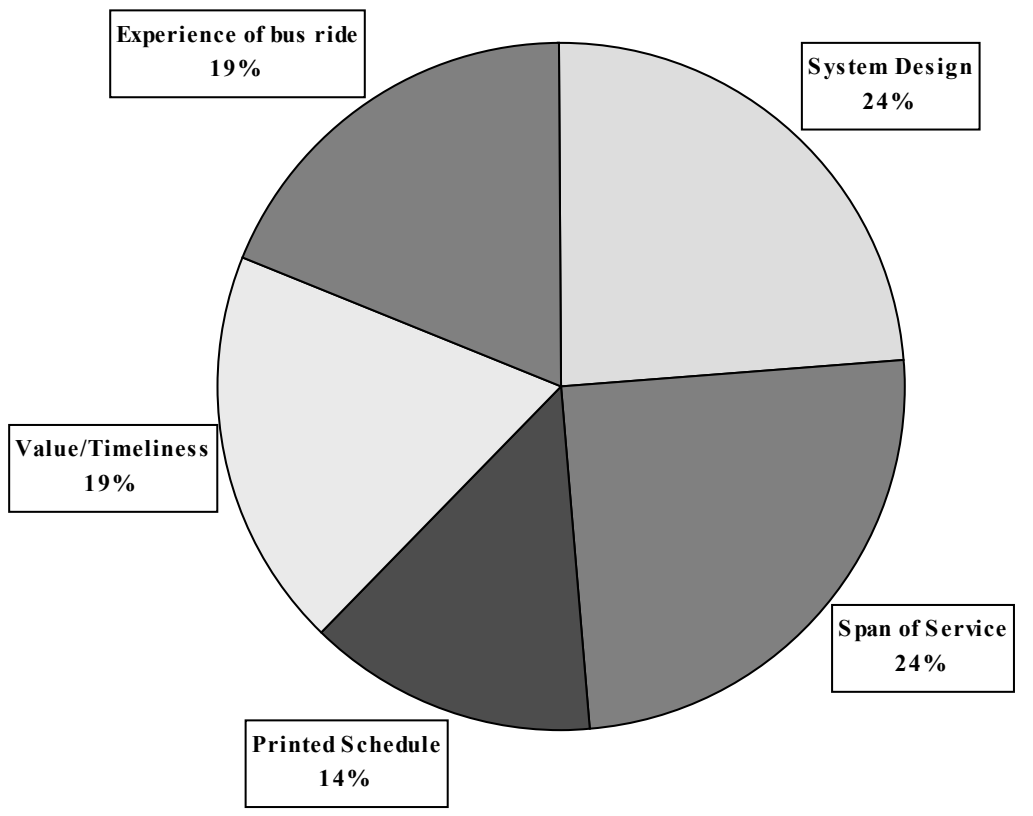

Figure 45: SCAT Customer Satisfaction Model 


\section{$\underline{\text { Demographic Analysis }}$}

\section{Income}

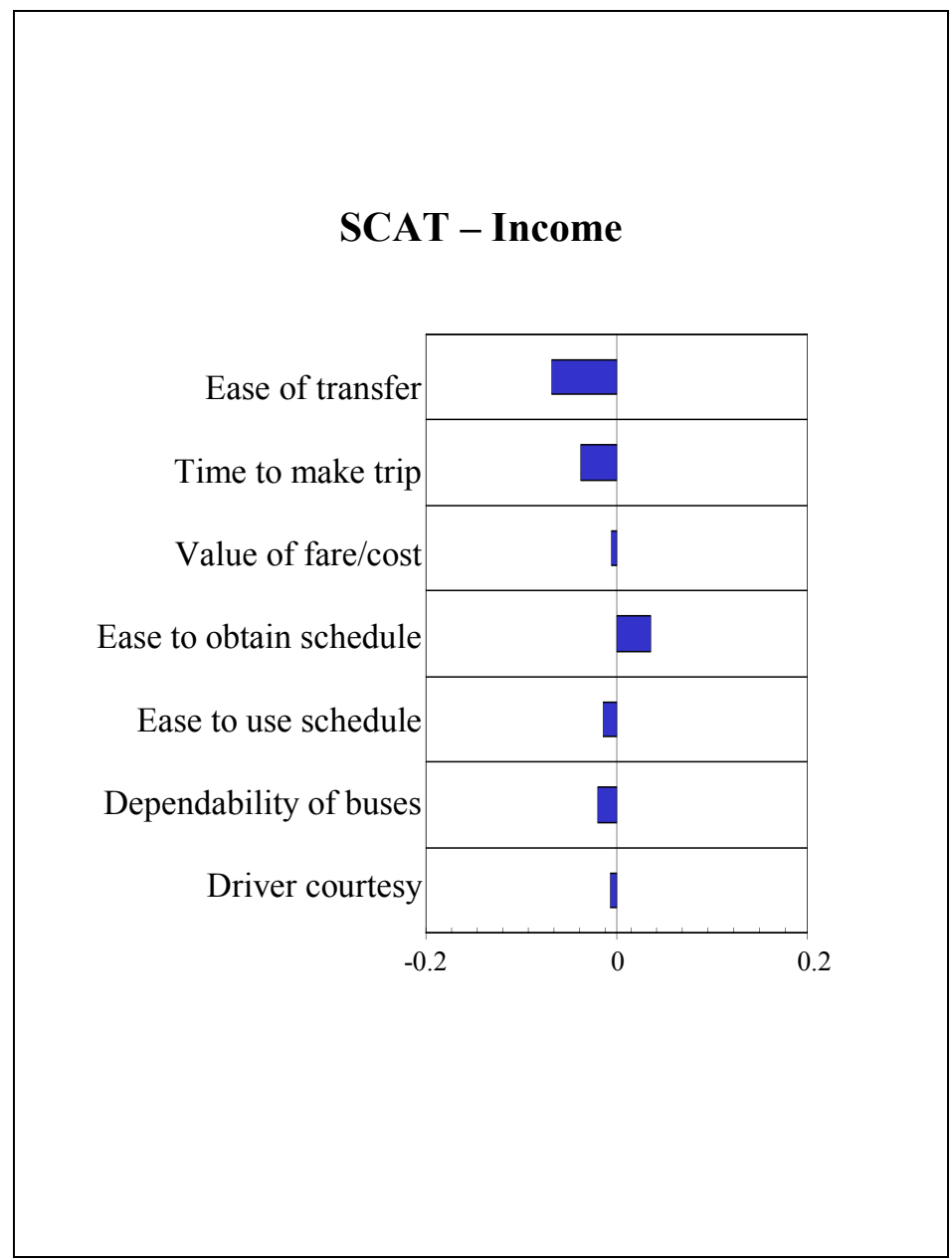

Figure 46: SCAT Correlation of income with demographic items

An analysis of non-linearly related items indicates that those with income of $\$ 25,000$ $\$ 50,000$ have the lowest levels of satisfaction with days and hours of service, and those with incomes of $\$ 15,000-\$ 50,000$ have the lowest levels of satisfaction with frequency of service.

The items with which lower income riders are more satisfied include ease of using schedules, on-time arrivals, ease of transfer, time to make trip, driver courtesy, and value of fare/cost. Higher frequency of riding the bus probably makes lower income riders more aware of bus routes. Therefore, it may be easier for them to transfer and utilize 
schedules. Lower income riders also probably use this familiarity to set more realistic expectations for these elements of the bus-riding than higher income riders. Thus the experiences that occur for which no prior expectation can reasonably be formed without prior use of the bus (such as on-time arrivals and issues relating to the driver) may be expected to get higher ratings among lower-income users. Unlike the overall income analysis of all Florida systems, SCAT's fare structure is perceived to be more valuable as income decreases. This may be due to the particular fare structure used by Sarasota. It might be worthwhile to compare the fare structures among Florida transit systems in order to get a better understanding of why this correlation is different from the statewide statistics. 


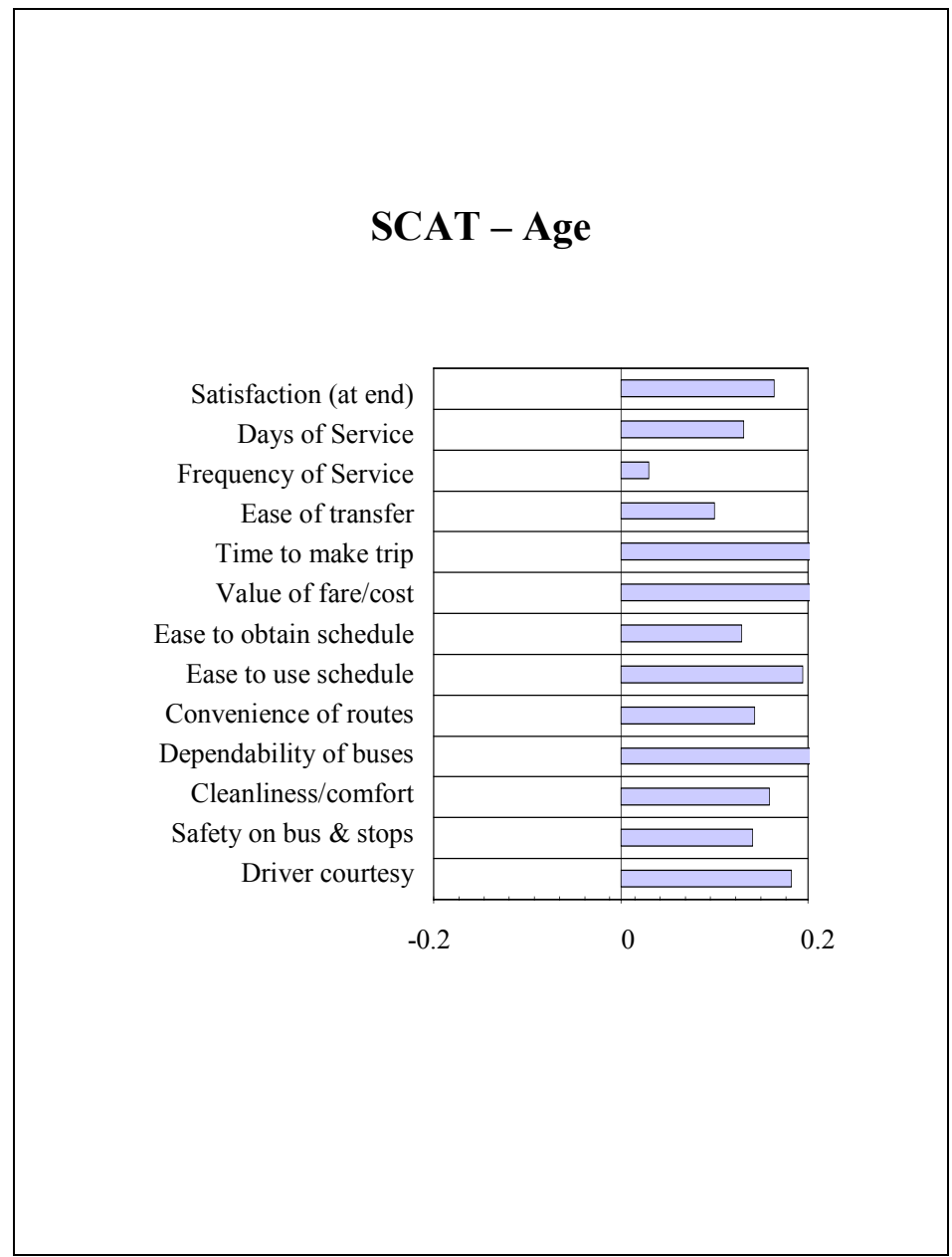

Figure 47: SCAT Correlation of age with demographic items

All of the satisfaction factors are positively correlated with age. Analysis of a nonlinearly-related item, hours of service, revealed essentially the same pattern. Older respondents are overwhelmingly satisfied with SCAT's transit service. As age increases, satisfaction increases for all categories. Different experiences between the older and younger individuals may cause the satisfaction differences between younger and older individuals. Individual time constraints and amount of leisure time can influence a passenger's satisfaction level. For instance, younger people are usually on a tighter schedule, such as arriving to work on time. Therefore, younger people will usually be more conscious of time delays. This can lead younger people to be less satisfied with frequency of service, ease of transfer, convenience of routes, and dependability. Also, younger passengers usually have less leisure time. Assuming that they would like to gain 
additional leisure time, their dissatisfaction with time to make trip is understandable. Moreover, younger riders tend to rate days of service with less satisfaction. Assuming that younger persons have less errand time during the weekday, they may perceive days of service to be unsatisfactory due to the fact that they need to take more trips on the weekend to fulfill all of their errands.

A combination of familiarity with surroundings as well as non-peak trips can lead to higher satisfaction ratings. Elderly people tend to utilize transit services in their particular neighborhood. Therefore, they are usually more familiar with their surroundings. Those who are more familiar with their surroundings tend to be generally more comfortable. Familiarity can lead older people to bestow higher ratings to driver courtesy, ease to obtain schedule, ease to use schedule, and safety factors. Riding a bus during non-peak trips increases the tendency of passengers to be more satisfied with the time to make trip and cleanliness/comfort of bus. For instance, a bus is perceived to be cleaner when there is less passenger congestion. Therefore, since older clients tend to ride the buses during non-peak service times, they tend to perceive the bus as being more clean/comfortable than younger passengers.

Finally, fare structure causes passengers to rate the value of transit differently. SCAT offers discount fares to senior citizens. Hence, it is not surprising that the value of trip satisfaction increases with age. 


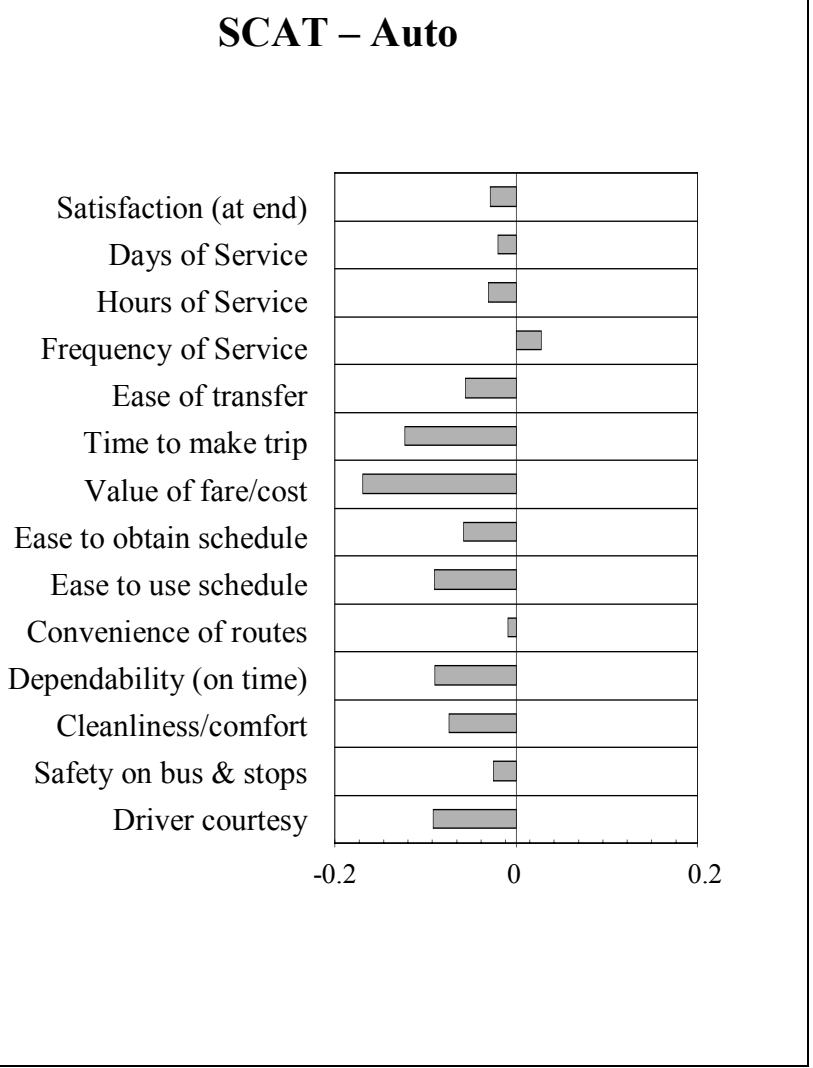

Figure 48: SCAT Correlation of auto ownership with demographic items

Auto ownership impacts these satisfaction levels because there is a difference between riding buses due to convenience versus riding them out of necessity. Households that own automobiles have the luxury of 24-hour transportation. In contrast, households without vehicles are constrained by transit service times, taxi costs, and/or friends' schedules. Therefore, it is not surprising to find that frequency of service was perceived to be more satisfactory by those who own automobiles.

In contrast to most systems' correlation outcomes, SCAT's surveys indicate that non-auto owners tend to perceive the transit in their area as more satisfactory. Negative correlations exist between number of automobiles and satisfaction in all but one category (frequency of service). Those without cars perceive transit as more valuable because they 
utilize it for all types of trips. Without transit, these passengers would not be able to work, go to school, run errands, and/or utilize public facilities. In contrast, customers who own automobiles tend to underestimate the value of transit. Automobile owners do not know the difficulties associated with trying to obtain rides for errands when transit is not available.

Familiarity with transit service may also cause negative correlations between automobile ownership and transit satisfaction. For instance, ease to obtain schedule, ease to use schedule, ease of transfer, driver courtesy, dependability of buses and safety are all issues that are negatively correlated with ownership. Much can be explained by the fact that those who do not have cars use transit the most. Therefore, familiarity with bus schedules and bus routes will lead persons without cars to be more satisfied. A negative correlation between ease of transfer and ownership suggests that since the non-owners frequently depend upon bus transfers, they are used to the routine method and therefore perceive it as easier. Those who use transit often will also be more familiar with bus drivers and bus conditions, thereby inciting a higher satisfaction with driver courtesy and safety. Also, the riders who use transit the most will have a more realistic view of bus dependability and allow for more time to make trip. Finally, lower income passengers perceived bus cleanliness and comfort to be more satisfactory than higher income passengers. This suggests that the riders who own automobiles are used to choosing their passengers. Auto owners may not be as comfortable with their fellow transit clientele and may be especially uncomfortable on crowded buses.

There are negative correlations between ownership and hours/days of service. One hypothesis is that service days and hours are severely curtailed in high-auto-ownership neighborhoods (consistent with income ratings). Two routes in high car ownership/income areas may justify this correlation. Routes 9 and 19 both contain twohour headways, which may lead passengers who own automobiles to be dissatisfied with service hours. Also, none of the routes that are run by SCAT offer Sunday service. Perhaps those without automobiles are more satisfied with days of service because routes in their areas tend to be longer (earlier start times and later end times) and more frequent during the week (Monday-Saturday).

Virtually zero correlation exists between convenience of routes and automobile ownership. Therefore, a detailed explanation is not given. 


\section{Ethnicity}

\begin{tabular}{|c|c|c|c|}
\hline & Table 25: SCAT Ethnicity & White & Black \\
\hline Sample size & & $\mathbf{7 9 8}$ & $\mathbf{1 6 8}$ \\
\hline & & & \\
\hline SQ05 & Frequency of Service & 3.74 & 4.08 \\
\hline SQ03 & Days of Service & 4.12 & 4.33 \\
\hline
\end{tabular}

Blacks are more satisfied with frequency of service and days of service. Both groups have high levels of satisfaction for both items. Level of service into neighborhoods that are predominantly black may be slightly higher, reflecting higher levels of ridership.

\section{Gender}

\begin{tabular}{|c|c|c|c|}
\hline & Table 26: SCAT Gender & Male & Female \\
\hline Sample size & & $\mathbf{4 9 3}$ & $\mathbf{5 8 3}$ \\
\hline & & & \\
\hline SQ29 & Driver courtesy & 4.14 & 4.41 \\
\hline SQ31 & Satisfaction (combined) & 4.16 & 4.36 \\
\hline
\end{tabular}

Males and females have very few differences in satisfaction with bus service. Women were more satisfied overall with bus service and more satisfied with driver courtesy. Since other differences were small, this suggests that driver courtesy plays a large role in satisfaction, at least between men and women in the SCAT service area. 


\section{$\underline{\text { Recommendations }}$}

From the customer satisfaction model, it is possible to construct an "importanceperformance" matrix that graphically illustrates current bus riders' perceptions of SCAT operations.

\section{Importance / Performance Matrix Sarasota Riders' perceptions of SCAT service}

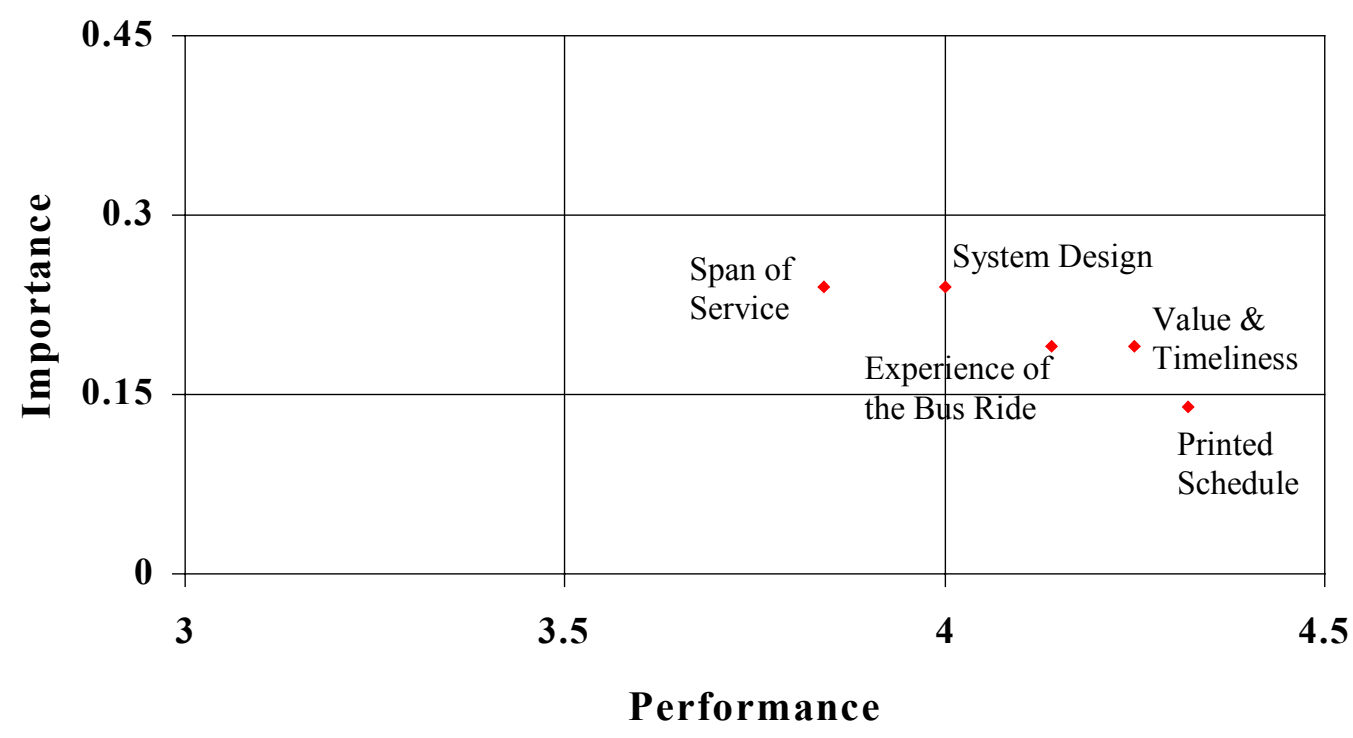

Figure 49: SCAT Importance/Performance matrix 
The chart has been divided into nine regions, reflecting various combinations of low, medium, and high performance and low, medium, and high importance. Borderline figures are interpreted as being in the higher of the importance categories they border on, but the lower of the performance categories. This provides the most conservative interpretation of the results. The interpretations of the chart regions are done as follows:

\begin{tabular}{|c|c|c|c|}
\hline \multicolumn{4}{|c|}{$\begin{array}{c}\text { Table } 27 \\
\text { Interpretations of SCAT Chart Regions }\end{array}$} \\
\hline \multicolumn{2}{|c|}{ Chart region } & \multirow[b]{2}{*}{ Interpretation } & \multirow[b]{2}{*}{ Areas } \\
\hline Importance & Performance & & \\
\hline Low & High & Possibly reduce focus on this area & Printed Schedule \\
\hline Low & Medium & Maintain performance - no action & \\
\hline Low & Low & Maintain performance - no action & \\
\hline Medium & High & Maintain performance - no action & $\begin{array}{l}\text { Experience of bus ride, } \\
\text { Value \& Timeliness }\end{array}$ \\
\hline Medium & Medium & Maintain performance - no action & $\begin{array}{l}\text { Span of Service, } \\
\text { System Design }\end{array}$ \\
\hline Medium & Low & Investigate for improvements & \\
\hline High & High & $\begin{array}{l}\text { Maintain performance - vigorous } \\
\text { quality checks, constant attention }\end{array}$ & \\
\hline High & Medium & Investigate for improvements & \\
\hline High & Low & Critical improvement area & \\
\hline
\end{tabular}

None of the items fall into a chart area that suggests immediate action is needed. 
According to the individual index and mean scores shown in Table I-G, SCAT is doing a good job with respect to the System Design factor category. The overall mean indicates that this category is one of SCAT's strengths. Moreover, all of the items indicate that SCAT's passengers perceive them better than average. SCAT scored high for each of the items in this category: "ease of transfer," "convenience of routes," frequency of service," "time to make trip," and "dependability of service. Therefore, SCAT should continue to provide its exemplary service in this category.

Table II-G reveals that SCAT's customers are satisfied with the Span of Service category, too. The overall mean was high, albeit lower than the System Design factor category. Also, compared to the other systems in Florida, SCAT's passengers are fairly satisfied with the items contained within the Span of Service category: "hours of service," "days of service," and "frequency of service." In fact, "frequency of service" obtained high accolades compared to the rest of the systems participating in this study. Again, no major alterations are needed to increase standards in this category.

Most of the demographic analysis notes differences between demographic group satisfaction based on either choice ridership or familiarity with the system, and thus does not lead to specific recommendations.

However, one item that was peculiar to Sarasota was the generally lower level of satisfaction with transit as auto ownership levels increased. This was interpreted as an underestimation of the true value of transit by auto-owning households, particularly multiple-auto-owning households. This suggests that a marketing campaign targeted to higher-income households to promote the value of transit may be a good step for SCAT to take.

Generally, satisfaction levels are extremely high. SCAT should be justly proud of their performance in providing customer satisfaction. 


\section{SCAT - Brevard County}

$\underline{\text { Factor Analysis }}$

Factor 1 - $\quad$ System Design

Major loadings - $\quad$ SQ4 Hours of service

SQ5 Frequency of service

SQ3 Days of service

SQ18 Convenience of routes

SQ10 Time to make trip

Minor loading - $\quad$ SQ8 Ease of transfer

SQ19 Dependability of service (on-time)

This construct includes customer satisfaction with hours of service, days of service, frequency of service and convenience of routes, and time to make trip. The slight loading of ease of transfers and on-time performance are further aspects of the customers' satisfaction with system design characteristics.

\begin{tabular}{|lc|c|}
\hline \multicolumn{3}{|c|}{ Table 28-I: Factor 1 - System Design } \\
\cline { 2 - 3 } & \multicolumn{2}{c|}{ Scores } \\
\cline { 2 - 3 } Item & Index & Mean \\
\hline Hours of Service & 101.87 & 3.70 \\
\hline Frequency of Service & 107.12 & 3.71 \\
\hline Days of Service & 102.48 & 4.24 \\
\hline Convenience of Routes & 100.42 & 4.01 \\
\hline Time to Make Trip & 112.84 & 4.20 \\
\hline Ease of Transfer & 110.92 & 4.33 \\
\hline Dependability of Service (On Time) & 109.87 & 4.29 \\
\hline Overall Mean & & 4.07 \\
\hline
\end{tabular}


Factor 4 - $\quad \underline{\text { Transfers }}$

Major loadings - $\quad$ SQ25 Safety on bus and stops

SQ8 Ease of transfer

Minor loadings - $\quad$ SQ10 Time to make trip

SQ18 Convenience of routes

SQ13 Ease to use schedule

The main element in this construct is a combination of safety and ease of transferring. The safety element in transferring probably relates to the safety the customer feels while waiting for their connecting service. Time to make trip, convenience of routes, and ease of using schedules all are common elements in evaluation of satisfaction with ease of transferring.

\begin{tabular}{|lc|c|}
\hline \multicolumn{3}{|c|}{ Table 28-II: Factor 4 - Transfers } \\
\hline \multicolumn{2}{|c|}{ Item } & Scores \\
\cline { 2 - 3 } & Index & Mean \\
\hline Safety on Bus and Stops & 106.90 & 4.64 \\
\hline Ease of Transfer & 110.92 & 4.33 \\
\hline Time to Make Trip & 112.84 & 4.20 \\
\hline Convenience of Routes & 100.42 & 4.01 \\
\hline Ease to Use Schedule & 107.16 & 4.41 \\
\hline Overall Mean & & $\mathbf{4 . 3 2}$ \\
\hline
\end{tabular}


Factor 5 - $\quad \underline{\text { Value }}$

Major loadings - $\quad$ SQ19 Dependability of service (on time)

SQ11 Value of fare/cost

Minor loading - $\quad$ SQ10 Time to make trip

This construct relates to customer satisfaction with value of fare/cost, on-time performance of buses, and time to make trip.

Time to make trip and buses running on time indicate that to a large extent, the time involved in the trip may be more of an issue than the cost of the fare.

\begin{tabular}{|lc|c|}
\hline \multicolumn{3}{|c|}{ Table 28-III: Factor 5 - Value } \\
\hline \multicolumn{2}{|c|}{ Item } & Scores \\
\cline { 2 - 3 } & Index & Mean \\
\hline Dependability of Service (On Time) & 109.87 & 4.29 \\
\hline Value of Fare/Cost & 112.34 & 4.61 \\
\hline Time to Make Trip & 112.84 & 4.20 \\
\hline Overall Mean & & 4.37 \\
\hline
\end{tabular}


Factor 7 - $\quad \underline{\text { Schedule }}$

Major loadings - $\quad$ SQ12 Ease to obtain schedule

SQ13 Ease to use schedule

Minor loadings - $\quad$ SQ8 Ease of transfer

SQ11 Value of fare/cost

The construct relates to customer experience using schedules. The main variables in this construct are satisfaction with the ease of using and obtaining the schedule. Ease of transferring also loads on to this factor as the schedules are a major component in making transfers easy to accomplish. The loading of the value component indicates that, as in many other systems, making schedules easy to obtain and easy to use are primary elements in improving value perceptions.

\begin{tabular}{|lc|c|}
\hline \multicolumn{3}{|c|}{ Table 28-IV: Factor 7 - Schedule } \\
\hline \multicolumn{2}{|c|}{ Item } & Scores \\
\cline { 2 - 3 } \multicolumn{1}{|c|}{ Index } & Mean \\
\hline Ease to Obtain Schedule & 108.42 & 4.44 \\
\hline Ease to Use Schedule & 107.16 & 4.41 \\
\hline Ease of Transfer & 110.92 & 4.33 \\
\hline Value of Fare/Cost & 112.34 & 4.61 \\
\hline Overall Mean & & $\mathbf{4 . 4 5}$ \\
\hline
\end{tabular}


Factor 9 - Experience of bus ride

Major loadings - $\quad$ SQ29 Driver courtesy

SQ21 Cleanliness/comfort

Minor loadings - $\quad$ SQ25 Safety on bus and stops

The construct encompasses all the major experiential elements involved in the bus ride perceptions of the bus stop (cleanliness and comfort), meeting the driver, cleanliness and comfort of the bus itself, and whatever perceptions of safety one might have while riding.

\begin{tabular}{|lc|c|}
\hline \multicolumn{2}{|c|}{ Table 28-V: Factor 9 - Experience of Bus Ride } \\
\hline \multirow{2}{*}{ Item } & Index & Mean \\
\cline { 2 - 3 } & 108.88 & 4.70 \\
\hline Driver Courtesy & 106.94 & 4.63 \\
\hline Cleanliness/Comfort & 106.90 & 4.64 \\
\hline Safety on Bus and Stops & & 4.65 \\
\hline Overall Mean & & \\
\hline
\end{tabular}




\section{$\underline{\text { Customer Satisfaction Model }}$}

The following chart indicates which of Brevard's factors are the most important. The most influential factors are those with the largest importance values. For instance, since System Design and Transfers have the highest importance values, SCAT should devote the most time and energy to these categories in order to improve customer satisfaction ratings. By looking at the individual items in each of these constructs, management can determine which items to focus on.

\section{Customer Satisfaction Model: SCAT - Brevard}

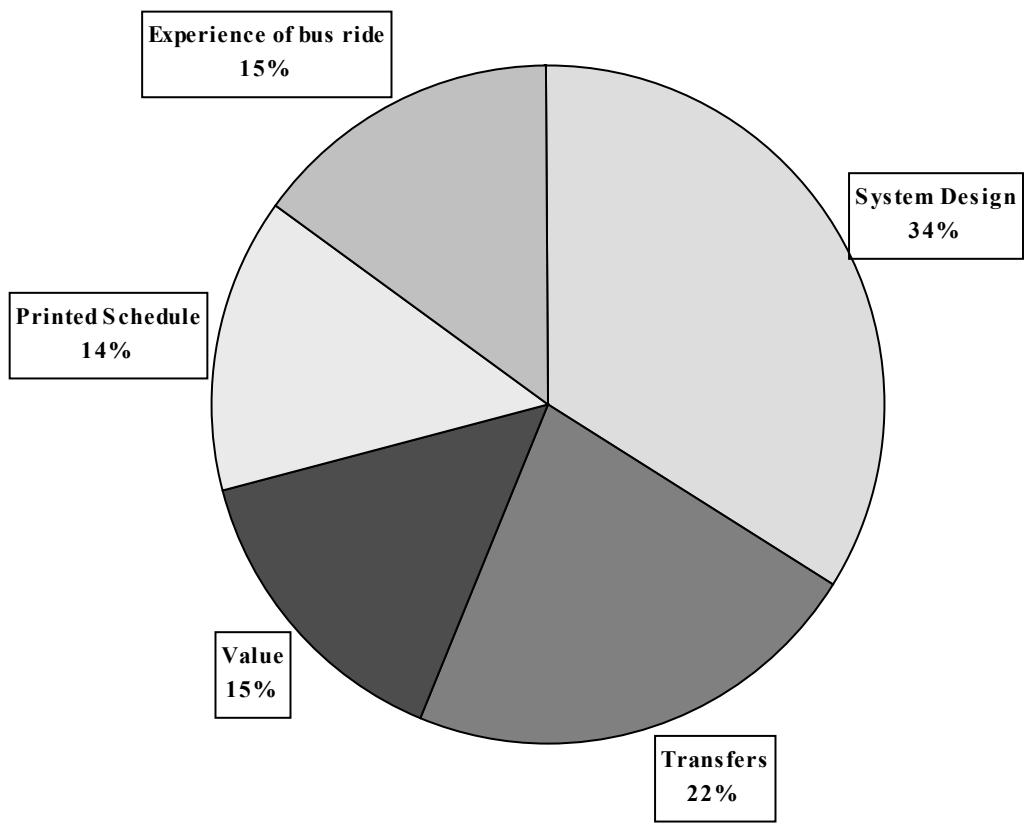

Figure 50: SCAT Brevard Customer Satisfaction Model 
$\underline{\text { Demographic Analysis }}$

\section{Income}

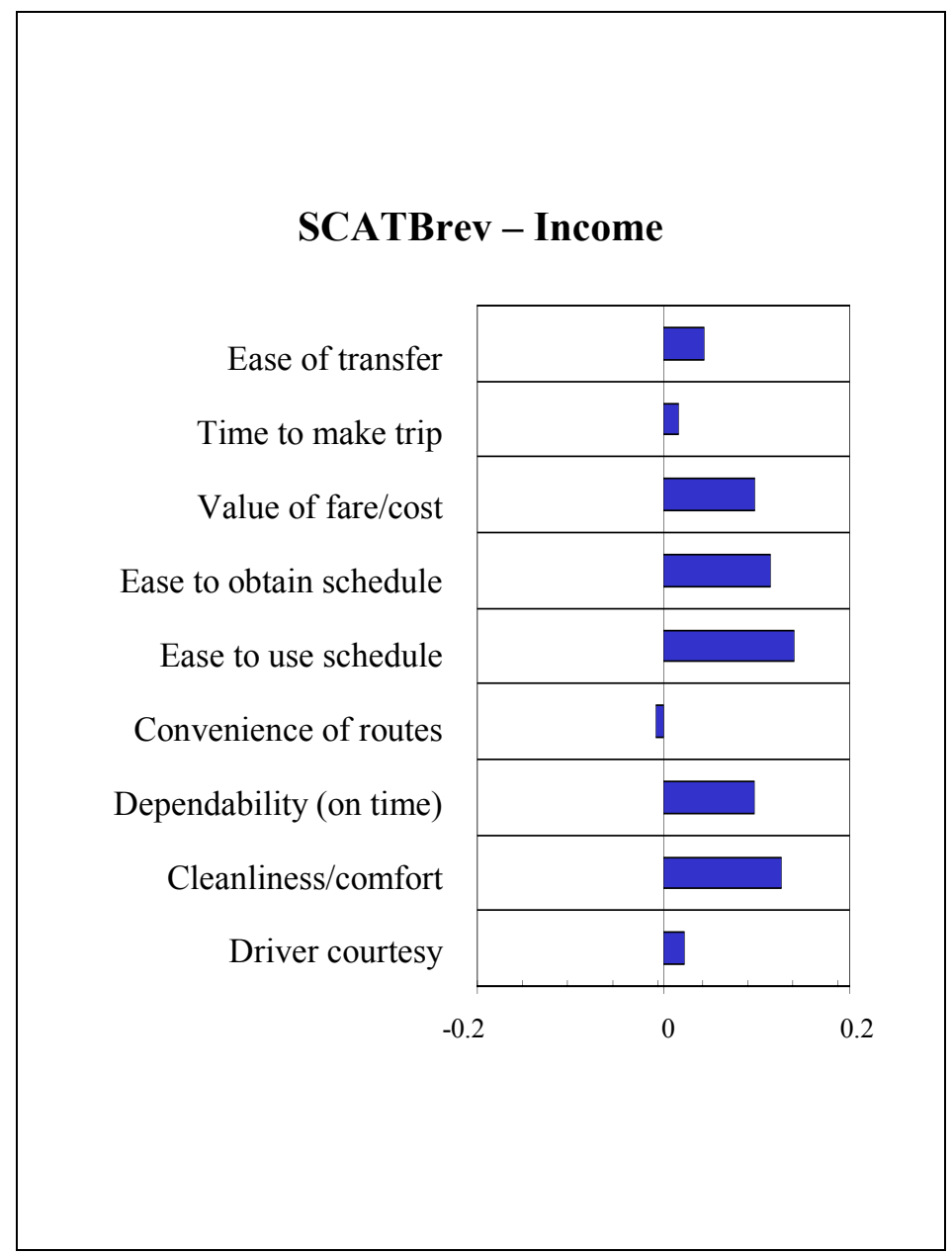

Figure 51: SCAT Brevard Correlation of income with satisfaction items

In all but one case, higher income riders are more satisfied with transit service than lower income riders. According to Brevard's survey results, those with a higher income tend to be more satisfied with comfort factors, dependability, ease of transfer, time to make trip, ease to obtain schedule, ease to use schedule, and fare value. The riders who have higher incomes are more likely to be choice riders and hence will only use the bus when it is convenient for them. Thus those of higher income who are potential riders who feel that the bus is not convenient (in terms of dependability, ease of transfer, time to make trip, etc.) will choose not to ride the bus and would therefore not be sampled. Lower income 
riders do not have this choice. Therefore, positive correlations exist between time to make trip, ease of transfer, and on-time performance and income.

Higher income individuals perceived comfort factors to be more satisfactory. They rated comfort/cleanliness and driver courtesy higher than low-income riders. These correlations probably relate to the fact that higher income individuals tend to ride buses in the nicer neighborhoods. Firstly, less passenger crowding causes comfort/cleanliness to be rated higher. Secondly, roads that are located within high-income neighborhoods tend to be maintained better. Therefore, bus drivers may be more relaxed and friendly when encountering a well-maintained stretch of road, thus leading to higher driver courtesy marks.

Survey results indicate that higher income groups rate Brevard's fare as being more valuable. This can be derived from a combination of circumstances (such as fare as a percentage of income and riding the bus only when it is convenient).

The one item with which lower income riders are more satisfied is convenience of routes. A Space Coast Area Transit representative indicated that this correlation is probably due to the fact that it is a small system with short, fixed routes. Since Space Coast's system of routes is no-frills, only those who find transit convenient will ride it. Therefore, it is suggested that the survey is catching only those who benefit from Space Coast's transit services.

Finally, the categories ease to obtain schedules and ease utilize schedules are positively related to income. The only viable explanation is that the sample size of higher income passengers is relatively small. Therefore, this correlation may not be able to catch all of the population's characteristics. 


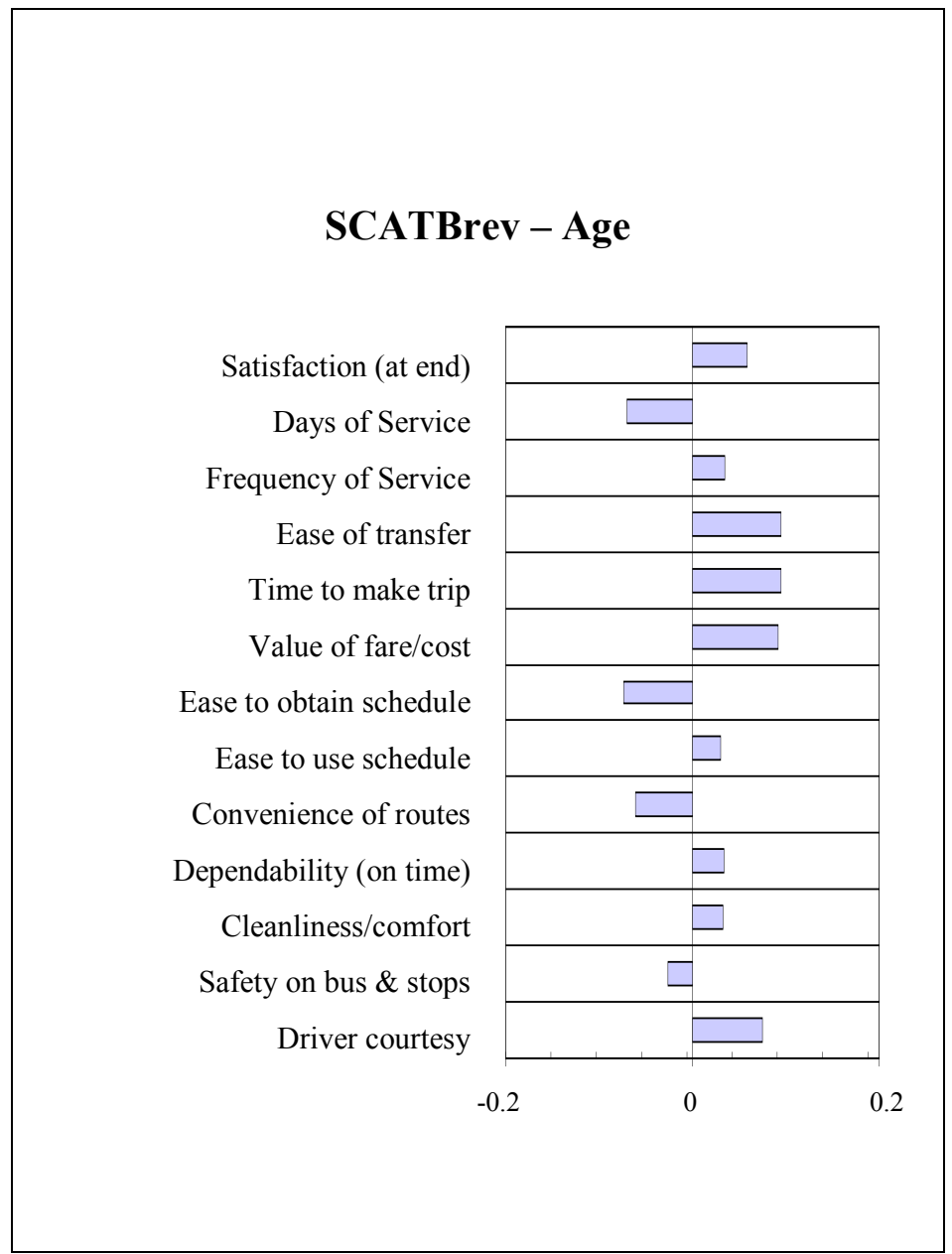

Figure 52: SCAT Brevard Correlation of age with satisfaction items

Most of the satisfaction factors are positively correlated with age. As age increases, satisfaction increases for all but four categories. Different experiences between the older and younger individuals may cause the satisfaction differences between younger and older individuals.

Individual time constraints and amount of leisure time can influence a passenger's satisfaction level. For instance, younger people are usually on a tighter schedule, such as arriving to work on time. Therefore, younger people will usually be more conscience of time delays. This can lead younger people to be less satisfied with frequency of service, ease of transfer, convenience of routes, and dependability. Also, younger passengers 
usually have less leisure time. Assuming that they would like to gain additional leisure time, their dissatisfaction with time to make trip is understandable.

A combination of familiarity with surroundings as well as non-peak trips can lead to higher satisfaction ratings. Elderly people tend to utilize transit services in their particular neighborhood. Therefore, they are usually more familiar with their surroundings. Those who are more familiar with their surroundings tend to be generally more comfortable. Familiarity can lead older people to bestow higher ratings to driver courtesy and ease to use schedule. Riding a bus during non-peak trips increases the tendency of passengers to be more satisfied with and cleanliness/comfort of bus. For example, a bus is perceived to be cleaner when there is less passenger congestion. Therefore, since older clients tend to ride the buses during non-peak service times, they tend to perceive the bus as being more clean/comfortable than younger passengers.

According to the survey, younger passengers are more satisfied with ease to obtain schedule. As with the income correlation above, the only viable explanation is that the sample size of higher income passengers is relatively small. Therefore, this age correlation result may not be able to catch all of the population's characteristics.

Fare structure causes passengers to rate the value of transit differently. Since Space Coast Transit Agency's fare is half of the regular price for senior citizens, it is not surprising that the value of trip satisfaction increases with age. Moreover, SCAT's senior citizens may want more days of service and more convenient routes to take advantage of their discount fare status. 


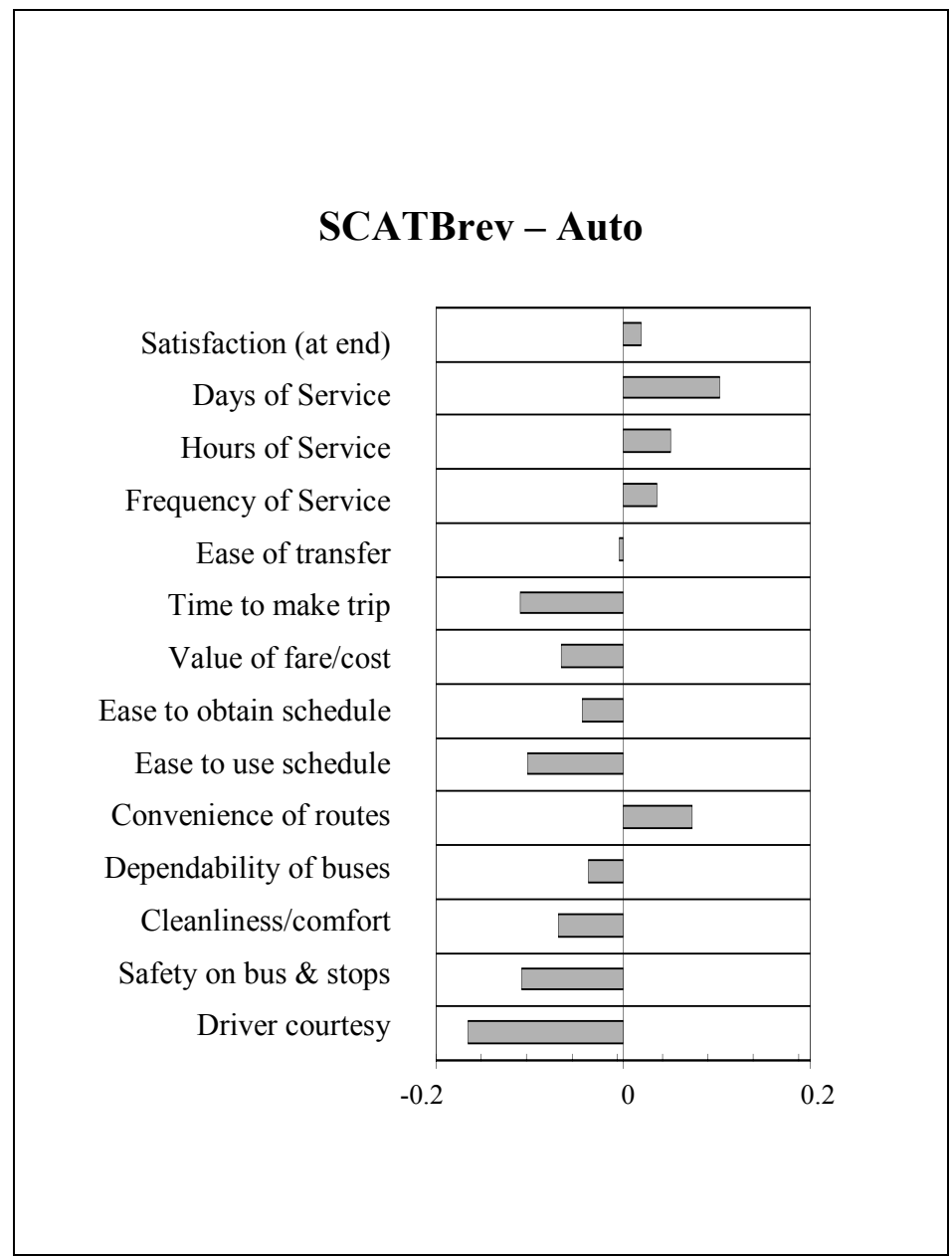

Figure 53: SCAT Brevard Correlation of auto ownership with satisfaction items

Overall, a positive correlation exists between number of automobiles and satisfaction with transit. Automobile owners ride transit only when it is convenient. Therefore, satisfaction with transit depends upon factors such as riding buses due to convenience versus riding them out of necessity. Since households that own automobiles have the luxury of 24-hour transportation, they are not hindered by transit schedules. In contrast, households without vehicles are constrained by transit service times, taxi costs, and/or friends' schedules. Therefore, persons without automobiles are less satisfied with days of service, hours of service, frequency of service, and convenience of routes.

Familiarity with transit service may also cause negative correlations between automobile ownership and transit satisfaction. For instance, ease to obtain schedule, ease to use 
schedule, ease of transfer, time to make trip, driver courtesy, dependability of buses and safety are all issues that are negatively correlated with ownership. Much can be explained by the fact that those who do not have cars use transit the most. Therefore, familiarity with bus schedules and bus routes will lead persons without cars to be more satisfied. A negative correlation between ease of transfer and ownership suggests that since the non-owners frequently depend upon bus transfers, they are used to the routine method and therefore perceive it as easier. Those who use transit often will also be more familiar with bus drivers and bus conditions, thereby inciting a higher satisfaction with driver courtesy and safety. Also, the riders who use transit the most will have a more realistic view of bus dependability and allow for more time to make trip. Finally, lower income passengers perceived bus cleanliness and comfort to be more satisfactory than higher income passengers. This suggests that the riders who own automobiles are used to choosing their passengers. Auto owners may not be as comfortable with their fellow transit clientele and may be especially uncomfortable on crowded buses. 


\section{Ethnicity}

Sample sizes were insufficient to permit meaningful analysis of variation across ethnic groups.

Gender

\begin{tabular}{|c|c|c|c|}
\hline & Table 29 - SCAT Brevard Gender & Male & Female \\
\hline Sample size & & $\mathbf{1 3 7}$ & $\mathbf{1 8 9}$ \\
\hline & & & \\
\hline SQ12 & Ease to obtain schedule & 4.18 & 4.65 \\
\hline SQ13 & Ease to use schedule & 4.24 & 4.53 \\
\hline SQ04 & Hours of Service & 3.55 & 3.82 \\
\hline SQ05 & Frequency of Service & 3.56 & 3.81 \\
\hline SQ03 & Days of Service & 4.10 & 4.34 \\
\hline
\end{tabular}

Females are more satisfied than males with all aspects of service for which there is a significant difference. These items include obtaining and using the schedule, and frequency, hours and days of service.

The schedule for SCAT is one of the best designed schedules of all Florida systems colorful, easy to read, with a lot of information about facilities at each stop. These design characteristics may help to make it easier to use and are less abstract than most other systems' schedules. 


\section{$\underline{\text { Recommendations }}$}

From the customer satisfaction model, it is possible to construct an "importanceperformance" matrix that graphically illustrates current bus riders' perceptions of SCAT operations.

\section{Importance / Performance Matrix} Space Coast Area Riders' perceptions of SCAT service

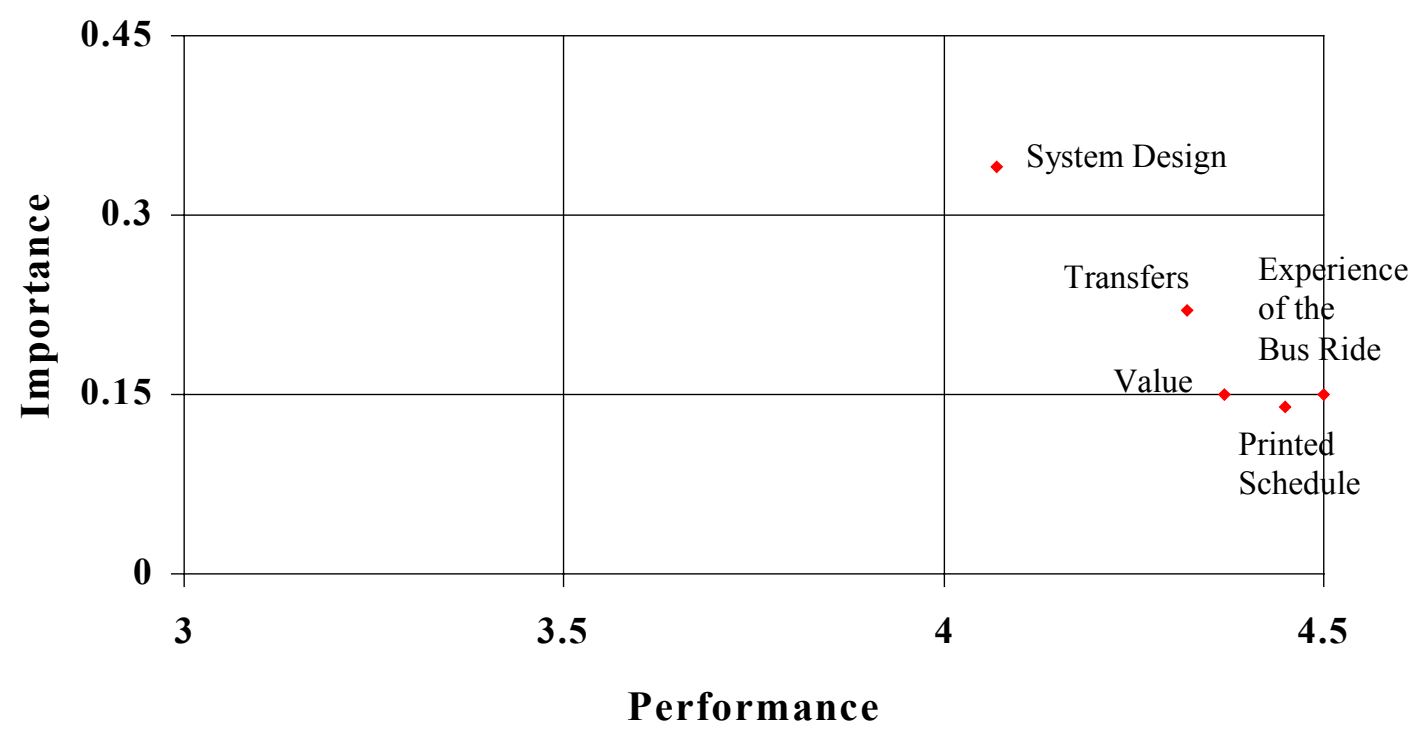

Figure 54: SCAT Brevard Importance/Performance Matrix 
The chart has been divided into nine regions, reflecting various combinations of low, medium, and high performance and low, medium, and high importance. Borderline figures are interpreted as being in the higher of the importance categories they border on, but the lower of the performance categories. This provides the most conservative interpretation of the results. The interpretations of the chart regions are done as follows:

\begin{tabular}{|c|c|c|c|}
\hline \multicolumn{4}{|c|}{$\begin{array}{c}\text { Table } 30 \\
\text { Interpretations of SCAT Chart Regions }\end{array}$} \\
\hline \multicolumn{2}{|c|}{ Chart region } & \multirow[b]{2}{*}{ Interpretation } & \multirow[b]{2}{*}{ Areas } \\
\hline Importance & Performance & & \\
\hline Low & High & $\begin{array}{l}\text { Possibly reduce focus on this } \\
\text { area }\end{array}$ & Printed Schedule \\
\hline Low & Medium & Maintain performance - no action & \\
\hline Low & Low & Maintain performance - no action & \\
\hline Medium & High & Maintain performance - no action & $\begin{array}{l}\text { Transfers, Value, } \\
\text { Experience of bus ride }\end{array}$ \\
\hline Medium & Medium & Maintain performance - no action & \\
\hline Medium & Low & Investigate for improvements & \\
\hline High & High & $\begin{array}{l}\text { Maintain performance - vigorous } \\
\text { quality checks, constant attention }\end{array}$ & System Design \\
\hline High & Medium & Investigate for improvements & \\
\hline High & Low & Critical improvement area & \\
\hline
\end{tabular}

None of the items fall into a chart area that suggests immediate action is needed. The System Design factor falls into an area requiring vigorous quality checks and constant attention. The items with lowest scores are Hours of service and Frequency of service. 
Frequency remains well above the Florida average. Hours of service is close to the Florida average. Some investigation could be made into whether there are specific areas in the Brevard county service area that might require extended service hours.

The individual index scores shown in the System Design table (I-H) show that SCAT is doing an excellent job with respect to the other systems in this study. Days of service, Convenience of routes, Time to make trip, Ease of transfer, and Dependability of service received relatively high marks, revealing that these areas do not need much reworking.

Finally, Table II-H indicates that SCAT Brevard's clients are usually more satisfied with Transfers than the other transit agencies' customers. In fact, all of the items within this category were rated higher than the state average. SCAT scored high for each of the items in this category: "safety on bus and stops," "ease of transfer," "convenience of routes," "time to make trip," and "ease to use service." Therefore, SCAT should continue to provide its exemplary service in this category, too.

Demographic correlations with satisfaction yielded few specific findings that could be translated into recommendations. It was noted in the course of conducting that analysis that the SCAT system is primarily a no-frills service, and probably only used by those who find it convenient. Those households that have low income may have less chance of being served by SCAT than in other areas. This begs the question of whether service is extensive enough of provide the transportation "safety net" that is such a large element of transit service.

Demographic analysis by ethnicity was not possible due to low sample sizes.

Females are more satisfied than males with all aspects of service for which there is a significant difference. These items include obtaining and using the schedule, and frequency, hours and days of service. The schedule for SCAT is one of the best designed schedules of all Florida systems - colorful, easy to read, with a lot of information about facilities at each stop. These design characteristics are less abstract than most other systems' schedules and may help to make it easier to use.

Overall, SCAT service is of excellent quality and should be maintained at its current level. The only question, as noted above, might be: is the reach of the service sufficient? 


\section{TALTRAN}

$\underline{\text { Factor Analysis }}$

Factor 1 - $\quad$ System Design

Major loadings - $\quad$ SQ4 Hours of service

SQ3 Days of service

SQ5 Frequency of service

SQ18 Convenience of routes

Minor loading - $\quad$ SQ8 Ease of transfer

This construct includes customer satisfaction with hours of service, days of service, frequency of service and convenience of routes. The slight loading of ease of transfers is another aspects of the customers' satisfaction with system design characteristics.

\begin{tabular}{|lc|c|}
\hline \multicolumn{2}{|c|}{ Table 31-I: TALTRAN Factor 1 - System Design } \\
\cline { 2 - 3 } & \multicolumn{2}{c|}{ Scores } \\
\cline { 2 - 3 } Item & Index & Mean \\
\hline Hours of Service & 94.37 & 3.42 \\
\hline Days of Service & 98.74 & 4.09 \\
\hline Frequency of Service & 98.23 & 3.40 \\
\hline Convenience of Service & 94.25 & 3.76 \\
\hline Ease of Transfer & 98.69 & 3.85 \\
\hline Overall Mean & 3.70 \\
\hline
\end{tabular}


Factor 5 - $\quad \underline{\text { Value }}$

Major loading - $\quad$ SQ11 Value of fare/cost

Minor loading - $\quad$ SQ10 Time to make trip

The primary component of this construct is customer perception of value. Time to make trip indicates that to a large extent, the time involved in the trip may be more of an issue than the cost of the fare.

\begin{tabular}{|lc|c|}
\hline \multicolumn{3}{|c|}{ Table 31-II: TALTRAN Factor 5 - Value } \\
\cline { 2 - 3 } & \multicolumn{2}{c|}{ Scores } \\
\cline { 2 - 3 } Item & Index & Mean \\
\hline Value of Fare/Cost & 100.55 & 4.13 \\
\hline Time to Make Trip & 99.60 & 3.71 \\
\hline Overall Mean & & $\mathbf{3 . 9 2}$ \\
\hline
\end{tabular}


Factor 7 - $\quad \underline{\text { Schedule }}$

Major loadings - $\quad$ SQ12 Ease to obtain schedule

SQ13 Ease to use schedule

Minor loadings - $\quad$ SQ8 Ease of transfer

The construct relates to customer experience using schedules. The main variable in this construct is satisfaction with the ease of using the schedule. Predictably, other variables loading on this factor are ease to obtain schedule and how regularly the buses arrive on time.

\begin{tabular}{|lc|c|}
\hline \multicolumn{2}{|c|}{ Table 31-III: TALTRAN Factor 7 - Schedule } \\
\cline { 2 - 3 } & \multicolumn{2}{c|}{ Scores } \\
\cline { 2 - 3 } Item & Index & Mean \\
\hline Ease to Obtain Schedule & 97.02 & 3.97 \\
\hline Ease to Use Schedule & 98.74 & 4.06 \\
\hline Ease of Transfer & 98.69 & 3.85 \\
\hline Overall Mean & 3.96 \\
\hline
\end{tabular}


Factor 9 - $\quad$ Experience of bus ride

Major loadings - $\quad$ SQ29 Driver courtesy

SQ25 Safety on bus and stops

SQ21 Cleanliness/comfort of buses and stops

SQ8 Ease of transfers

Minor loadings - $\quad$ SQ3 Days of service

SQ13 Ease to use schedule

The construct encompasses all the major experiential elements involved in the bus ride perceptions of the bus stop (cleanliness and comfort), meeting the driver, cleanliness and comfort of the bus itself, transferring, and whatever perceptions of safety one might have while riding.

Reading the schedule can also be seen as part of the experience of planning and making the trip.

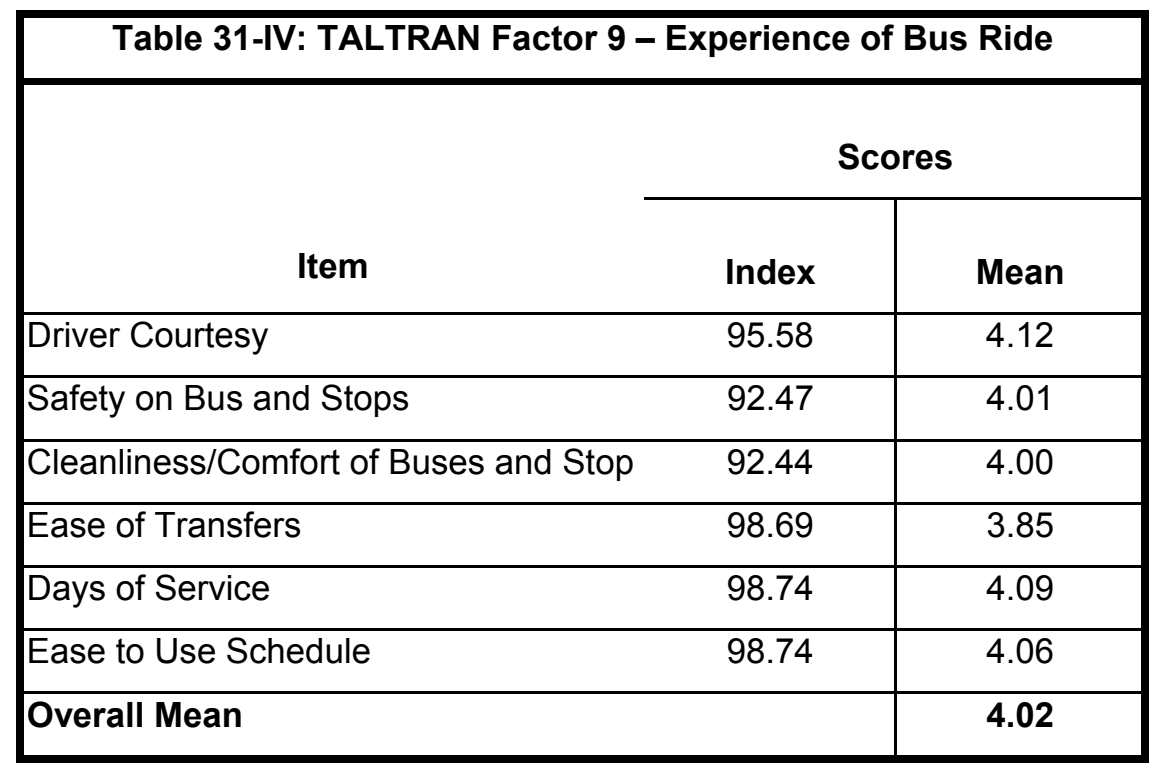


Factor 10 - Timeliness of Service

Major loadings - $\quad$ SQ19 Dependability of service (on time)

SQ10 Time to make trip

Minor loadings - $\quad$ SQ5 Frequency of service

SQ18 Convenience of routes

This construct relates to issues of dependability of buses running on time and time to make trip. Also, frequency of service and convenience of routes were slightly loaded on to this factor. These variables are connected with the customer's ability to arrive at their destination in a timely manner.

\begin{tabular}{|c|c|c|}
\hline \multirow[b]{3}{*}{ Item } & imeline & rvice \\
\hline & \multicolumn{2}{|c|}{ Scores } \\
\hline & Index & Mean \\
\hline Dependability of Service (On Time) & 86.96 & 3.39 \\
\hline Time to Make Trip & 99.60 & 3.71 \\
\hline Frequency of Service & 98.23 & 3.40 \\
\hline Convenience of Routes & 94.25 & 3.76 \\
\hline Overall Mean & & 3.57 \\
\hline
\end{tabular}




\section{$\underline{\text { Customer Satisfaction Model }}$}

The following chart indicates which of TALTRAN's factors are the most important. The most influential factors are those with the largest importance values. For instance, since System Design, Experience of Bus Ride, and Timeliness of service have the highest importance values, TALTRAN should devote the most time and energy to these categories in order to improve customer satisfaction ratings. By looking at the individual items in each of these three constructs, management can determine which items to focus on.

\section{Customer Satisfaction Model: TALTRAN}

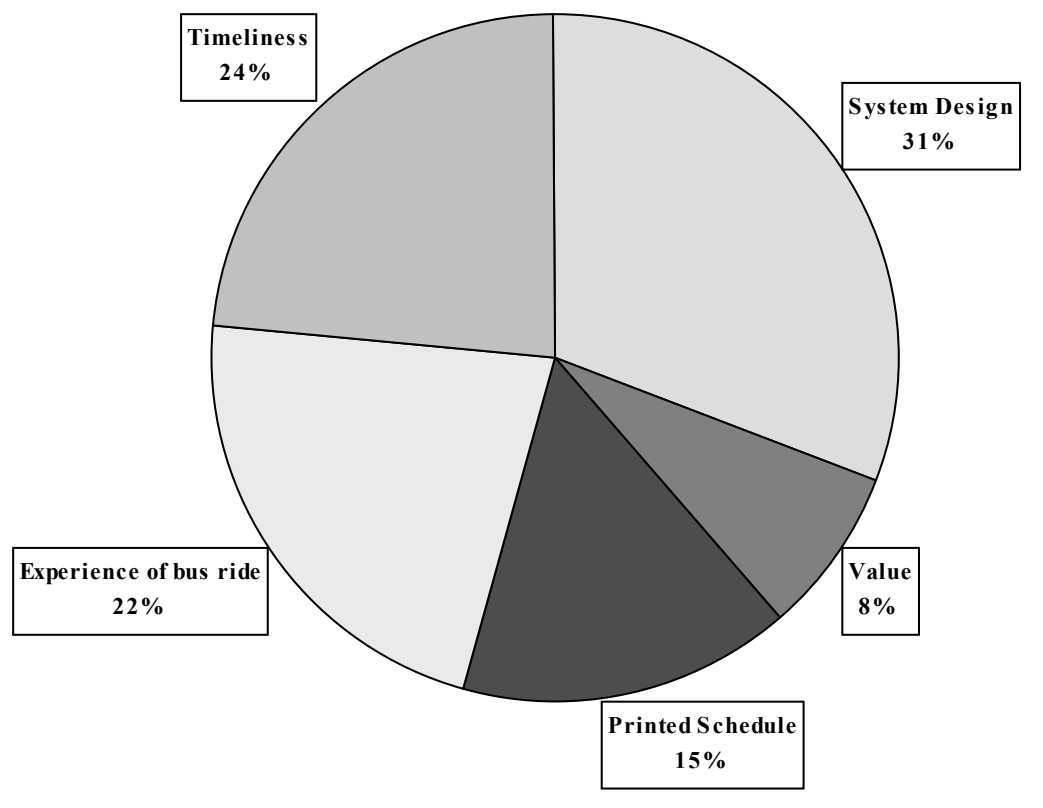

Figure 55: TALTRAN Customer Satisfaction Model 
$\underline{\text { Demographic Analysis }}$

\section{Income}

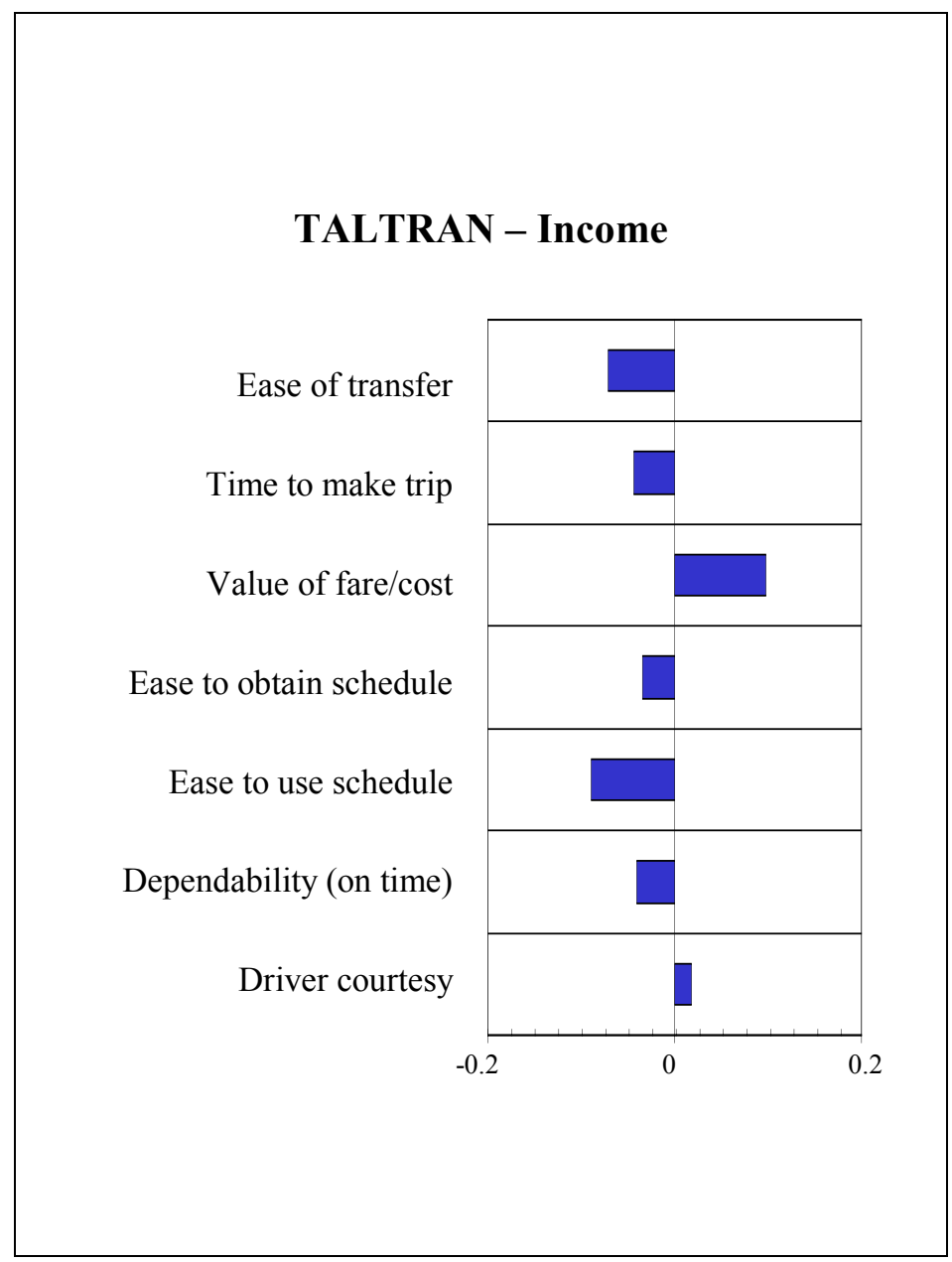

Figure 56: TALTRAN Correlation of income with satisfaction items

An analysis of non-linearly related items revealed that those with incomes of $\$ 25,000$ $\$ 50,000$ were most satisfied with days and hours of service and frequency of service.

The items with which lower income riders are more satisfied include ease of obtaining schedules, ease of using schedules, ease of transfer, on-time arrivals, and time to make trip. Higher frequency of riding the bus probably makes lower income riders more aware of where to obtain schedules. Lower income riders also probably use this familiarity to set more realistic expectations for these elements of the bus-riding than higher income riders. Thus the experiences that occur for which no prior expectation can reasonably be 
formed without prior use of the bus (such as on-time arrivals, ease of using schedules, time to make trip, and ease of transfers) may be expected to get higher ratings among lower-income users.

Higher income individuals perceived driver courtesy and value of fare to be more satisfactory. Satisfaction with driver courtesy probably relates to the fact that higher income individuals tend to ride buses in the nicer neighborhoods. Roads that are located within high-income neighborhoods tend to be maintained better. Therefore, drivers may be more relaxed and friendly when encountering a well-maintained stretch of road. The transit fare being perceived as a better value can be derived from a combination of circumstances (such as fare as a percentage of income and riding the bus only when it is convenient). 


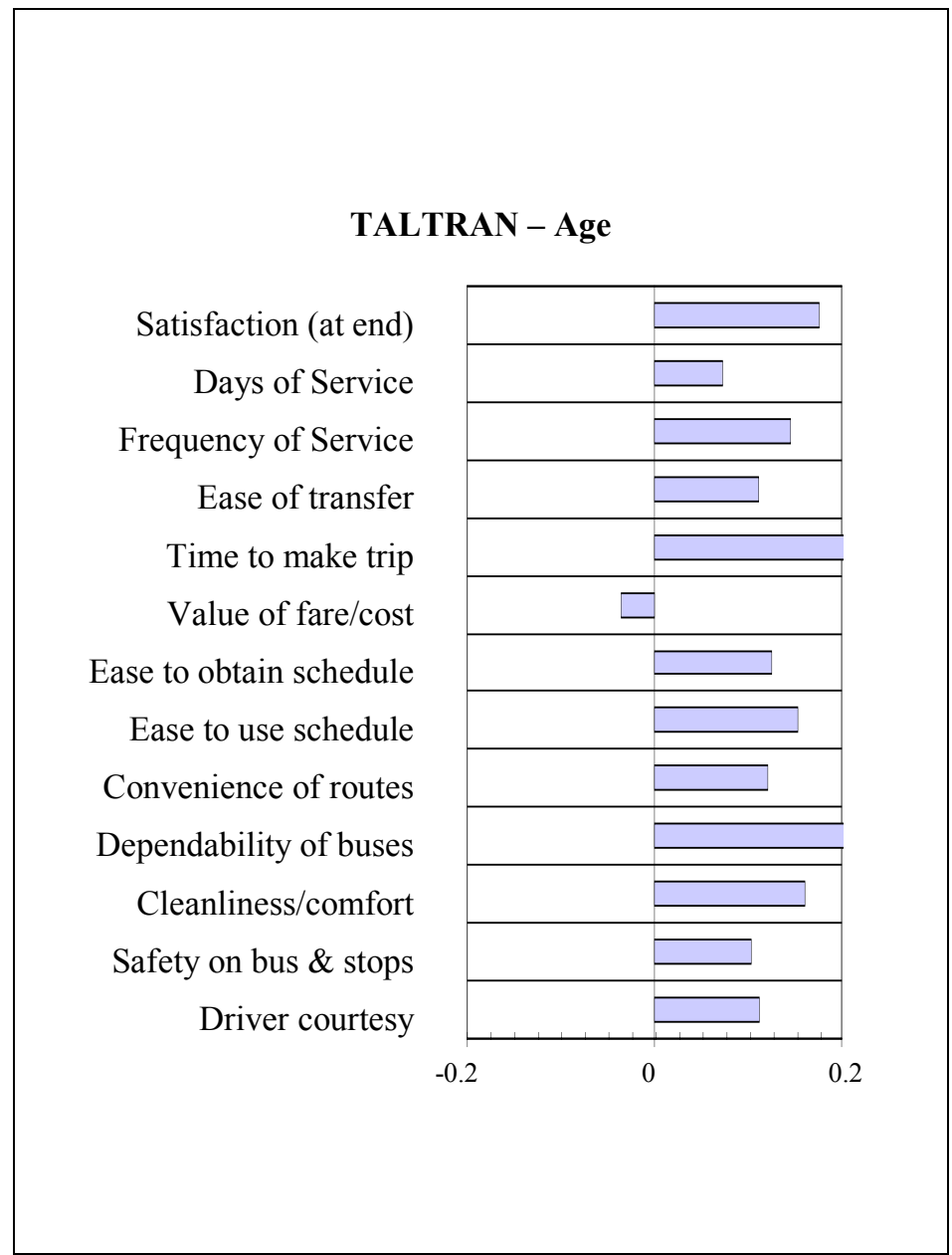

Figure 57: TALTRAN Correlation of age with satisfaction items

A non-linearly related item, satisfaction with hours of service, was generally positively correlated with age.

Almost all of the satisfaction factors are positively correlated with age. Older respondents are overwhelmingly satisfied with transit service. As age increases, satisfaction increases for all but one category, value of fare/cost. Different experiences between the older and younger individuals may cause the satisfaction differences between younger and older individuals.

Individual time constraints and amount of leisure time can influence a passenger's satisfaction level. For instance, younger people are usually on a tighter schedule, such as 
arriving to work on time. Therefore, younger people will usually be more conscience of time delays. This can lead younger people to be less satisfied with frequency of service, ease of transfer, convenience of routes, and dependability. Also, younger passengers usually have less leisure time. Assuming that they would like to gain additional leisure time, their dissatisfaction with time to make trip is understandable. Moreover, younger riders tend to rate days of service with less satisfaction. Assuming that younger persons have less errand time during the weekday, they may perceive days of service to be unsatisfactory due to the fact that they need to take more trips on the weekend to fulfill all of their errands.

A combination of familiarity with surroundings as well as non-peak trips can lead to higher satisfaction ratings. Elderly people tend to utilize transit services in their particular neighborhood. Therefore, they are usually more familiar with their surroundings. Those who are more familiar with their surroundings tend to be generally more comfortable. Familiarity can lead older people to bestow higher ratings to driver courtesy, ease to obtain schedule, ease to use schedule, and safety factors. Riding a bus during non-peak trips increases the tendency of passengers to be more satisfied with the cleanliness and comfort of bus. For example, a bus is perceived to be cleaner when there is less passenger congestion. Therefore, since older clients tend to ride the buses during non-peak service times, they tend to perceive the bus as being more clean/comfortable than younger passengers.

Finally, fare structure causes passengers to rate the value of transit differently. TALTRAN offers discount fares to senior citizens and college students. Senior citizens can obtain a half-price discount. Moreover, TALTRAN'S contracts with FAMU and FSU provide a fare free zones Monday through Friday during each semester. It also has a summer youth pass that offers substantial savings. Hence, unlike most of the other Florida systems, satisfaction with fares decreases with age. 


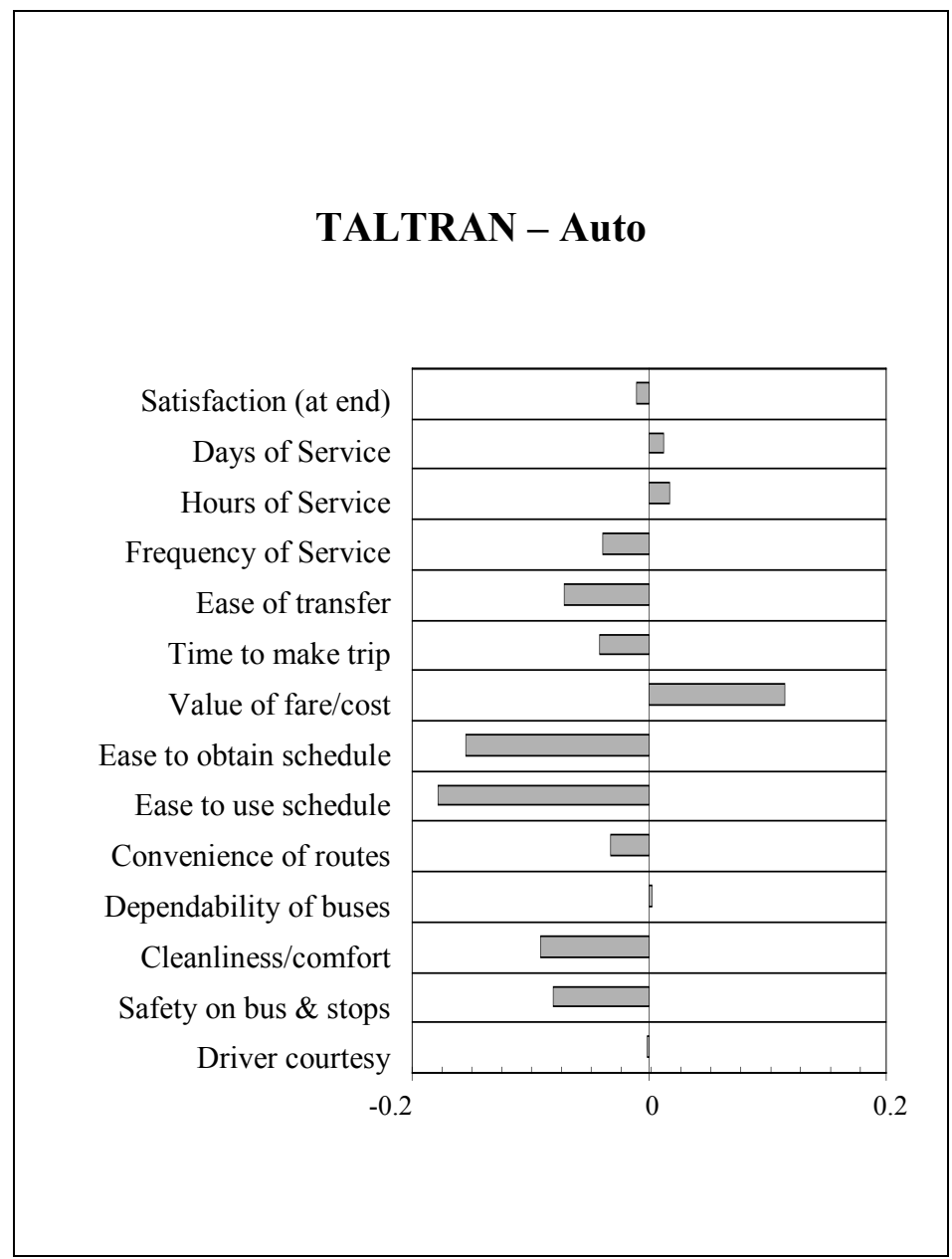

Figure 58: TALTRAN Correlation of auto ownership with satisfaction items

Auto ownership impacts these satisfaction levels because there is a difference between riding buses due to convenience versus riding them out of necessity. Not surprisingly, automobile owners tended to be more satisfied with days and hours of service.

Households that own automobiles have the luxury of 24-hour transportation. In contrast, households without vehicles are constrained by transit service times, taxi costs, and/or friends' schedules. Therefore, persons without automobiles are less satisfied with TALTRAN's service begin and end times. Again, since riders who own automobiles choose to ride transit only when it is convenient, they tend to be more satisfied with the time it takes to make trip. 
Familiarity with transit service can cause negative correlations between automobile ownership and transit satisfaction. For instance, ease to obtain schedule, ease to use schedule, ease of transfer, driver courtesy, and safety are all issues that are negatively correlated with ownership. Much can be explained by the fact that those who do not have cars use transit the most. Therefore, familiarity with bus schedules and bus routes will lead persons without cars to be more satisfied. A negative correlation between ease of transfer and ownership suggests that since the non-owners frequently depend upon bus transfers, they are used to the routine method and therefore perceive it as easier. Those who use transit often will also be more familiar with bus drivers and bus conditions, thereby inciting a higher satisfaction with driver courtesy and safety.

Unlike most of the transit systems in Florida, TALTRAN's satisfaction of the value of fare/cost is positively correlated with number of automobiles in household. This is probably due to TALTRAN's unusual fare structure. Those who are affiliated with the universities in the area probably own cars. Yet, they pay nothing to ride the buses in the fare free areas, thereby leading to a positive correlation.

There is a negative correlation between frequency of service and auto ownership. Unlike Gainesville's college town routes, Tallahassee's campus routes stay near campus. In fact, TALTRAN's campus passengers must go to a transfer station in order to get to most of Tallahassee's apartment complexes, shopping malls, and entertainment places off campus. Moreover, neighborhoods with high auto ownership/high income residents tend to ask for less service. Therefore, it is not surprising to note that those who live in these neighborhoods and ride buses may be disappointed with RTS' service frequency.

Automobile owners may have extremely high expectations of transit service. For instance, owners tend to be less satisfied with frequency of service and cleanliness/comfort of bus. Finally, lower income passengers perceived bus cleanliness and comfort to be more satisfactory than higher income passengers. This suggests that the riders who own automobiles are used to choosing their passengers. Auto owners may not be as comfortable with their fellow transit clientele and may be especially uncomfortable on crowded buses.

Note that virtually no correlations exist between auto ownership and dependability of buses and driver courtesy. Therefore, no explanation is given for these factors. 


\section{Ethnicity}

\begin{tabular}{|c|c|c|c|}
\hline & Table 32: TALTRAN Ethnicity & White & Black \\
\hline Sample size & & $\mathbf{2 6 5}$ & $\mathbf{8 6 6}$ \\
\hline & & & \\
\hline SQ08 & Ease of transfer & 4.03 & 3.81 \\
\hline SQ29 & Driver courtesy & 4.28 & 4.04 \\
\hline SQ04 & Hours of Service & 3.63 & 3.37 \\
\hline SQ32 & Convenience of routes (combined SQ 6 \& 18) & 3.99 & 3.70 \\
\hline SQ34 & Cleanliness/comfort (combined SQ 20 \& 21) & 4.24 & 3.91 \\
\hline SQ10 & Time to make trip & 3.98 & 3.62 \\
\hline SQ31 & Satisfaction (combined) & 4.14 & 3.77 \\
\hline SQ11 & Value of fare/cost & 4.45 & 4.03 \\
\hline
\end{tabular}

In most cases, whites have higher levels of satisfaction than blacks. This is probably related to many of the same factors that create higher satisfaction levels among higher income riders - mainly because they can be choice riders and are more apt to use the transit when it is convenient, but always having the option to drive where transit service does not meet their need for convenience and speed.

\section{Gender}

\begin{tabular}{|c|c|c|c|}
\hline & Table 33: TALTRAN Gender & Male & Female \\
\hline Sample size & & $\mathbf{4 6 6}$ & $\mathbf{7 3 5}$ \\
\hline & & & \\
\hline SQ11 & Value of fare/cost & 4.01 & 4.22 \\
\hline SQ13 & Ease to use schedule & 3.96 & 4.14 \\
\hline & Dependability of buses (on time) (combined SQ 9 \& & & \\
SQ33 & $19)$ & 3.53 & 3.33 \\
\hline
\end{tabular}

For most items, males and females have equal levels of satisfaction. Women provide slightly higher ratings for value of fare and ease of using schedule, while men have higher levels of satisfaction with on-time performance. It's possible that since women understand the schedule better, they may be more keenly aware of late arrivals. Also, since they may use the bus more throughout the day, they may have more opportunities to be exposed to late bus arrivals. 


\section{$\underline{\text { Recommendations }}$}

From the customer satisfaction model, it is possible to construct an "importanceperformance" matrix that graphically illustrates current bus riders' perceptions of SCAT operations.

\section{Importance / Performance Matrix Tallahassee Riders' perceptions of TALTRAN service}

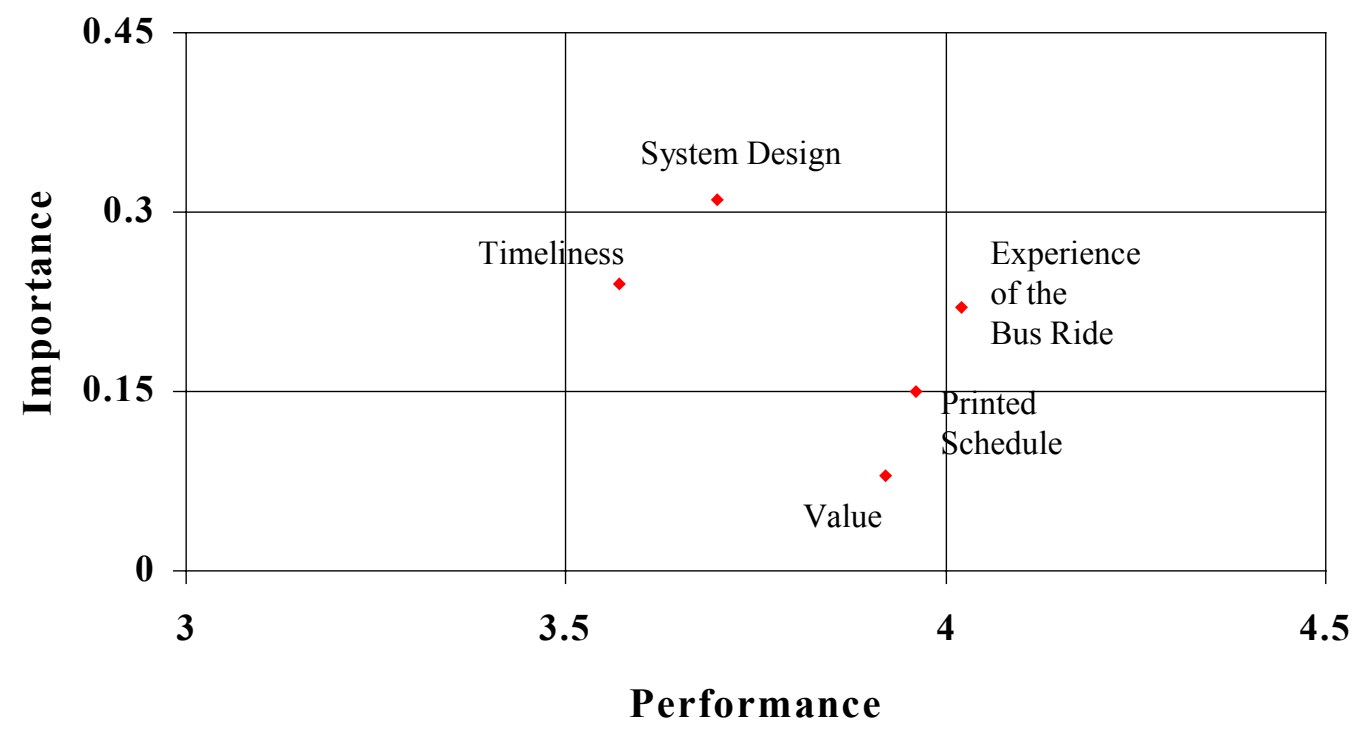

Figure 59: TALTRAN Importance/Performance Matrix

The chart has been divided into nine regions, reflecting various combinations of low, medium, and high performance and low, medium, and high importance. Borderline figures are interpreted as being in the higher of the importance categories they border on, but the lower of the performance categories. This provides the most conservative interpretation of the results. The interpretations of the chart regions are done as follows: 


\begin{tabular}{|c|c|c|c|}
\hline \multicolumn{4}{|c|}{$\begin{array}{c}\text { Table } 34 \\
\text { Interpretations of TALTRAN Chart Regions }\end{array}$} \\
\hline \multicolumn{2}{|c|}{ Chart region } & \multirow[b]{2}{*}{ Interpretation } & \multirow[b]{2}{*}{ Areas } \\
\hline Importance & Performance & & \\
\hline Low & High & Possibly reduce focus on this area & \\
\hline Low & Medium & Maintain performance - no action & Value \\
\hline Low & Low & Maintain performance - no action & \\
\hline Medium & High & Maintain performance - no action & Experience of bus ride \\
\hline Medium & Medium & Maintain performance - no action & $\begin{array}{l}\text { Timeliness, } \\
\text { Printed Schedule }\end{array}$ \\
\hline Medium & Low & Investigate for improvements & \\
\hline High & High & $\begin{array}{l}\text { Maintain performance - vigorous } \\
\text { quality checks, constant attention }\end{array}$ & \\
\hline High & Medium & Investigate for improvements & System Design \\
\hline High & Low & Critical improvement area & \\
\hline
\end{tabular}


System Design has a major impact on TALTRAN's customer satisfaction ratings and falls into the chart are for "Investigate for improvements." Therefore, this category is very important to the overall satisfaction ratings. Scores that are particularly low are reported below:

\begin{tabular}{|ll|c|}
\hline & \multicolumn{2}{c|}{ Scores } \\
\cline { 2 - 3 } \multicolumn{1}{c}{ Item } & Index & Mean \\
\hline Hours of Service & 94.37 & 3.42 \\
\hline Frequency of Service & 98.23 & 3.40 \\
\hline Convenience of Service & 94.25 & 3.76 \\
\hline
\end{tabular}

The individual index scores shown in Table I-I show that TALTRAN is doing a good job with respect to the other systems in this study. However, according to TALTRAN's System Design overall mean, its passengers are less satisfied with System Design when compared to the other categories. "Days of service," "convenience of routes," and "ease of transfer" received better marks, revealing that these areas do not need much reworking. In contrast, "Hours of service" and "frequency of service" received lower marks, indicating that these areas may need to be reevaluated.

There are two other areas that potentially may bear further investigation. Table IV-I indicates that Tallahassee's clients are usually satisfied with Experience of the Bus Ride. TALTRAN scored fairly high compared to other systems within the state of Florida. Each of the items in this Experience of the Bus Ride category included high indices: "driver courtesy," "safety on bus and stops," "cleanliness/comfort of buses and stop," "ease of transfers," "days of service," and "ease to use schedule." However, compared to all of the other items in this category, "ease of transfer" received low marks from TALTRAN's passengers. Therefore, resources could be utilized to increase the satisfaction of this item (i.e. alleviate the number of transfers needed to cross town; reconsider transfer ticket policies).

Timeliness (Table V-I) is another area that may need further development. Compared to the rest of TALTRAN's survey items, these items have the lowest overall score. The "dependability of service" item scored the lowest of all the other items in this category. 
On Time performance seems to be an issue that needs to be reevaluated. Perhaps traffic conditions have changed in Tallahassee so that the bus schedule is not appropriate currently. "Time to make trip," "frequency of service," and "convenience of routes" contained low scores, too. A few suggestions include adding buses to schedule, reevaluating routes, utilizing special-use lanes, adding express routes, et cetera.

Demographic correlations with satisfaction yielded few specific findings that could be translated into recommendations. Most of the findings were related either to choice use or familiarity, and did not provide a recommendation. There is an indication that perception of value decreases with age, which is unusual in Florida. This is probably due to TALTRAN fare structure regarding FSU students. 


\section{VOTRAN}

$\underline{\text { Factor Analysis }}$

Factor 1 - $\quad \underline{\text { System Design }}$

Major loadings - $\quad$ SQ7 Number of transfer

SQ8 Ease of transfer

Minor loadings - $\quad$ SQ6 Ability to get where you want to go

This construct relates to customer satisfaction with the number of transfers, ease of transfers and with a minor loading of the variable ability to get where you want to. Clearly the number and ease of making transfers has a major impact on customer perception of being able to get where they want to go.

\begin{tabular}{|lc|c|}
\hline \multicolumn{2}{|c|}{ Table 35-I: VOTRAN Factor 1 - System Design } \\
\cline { 2 - 3 } & \multicolumn{2}{c|}{ Scores } \\
\cline { 2 - 3 } & Indem & Mean \\
\hline Number of Transfer & 108.45 & 3.93 \\
\hline Ease of Transfer & 110.74 & 4.32 \\
\hline Ability to Get Where You Want to Go & 111.18 & 4.35 \\
\hline \multirow{2}{*}{ Overall Mean } & $\mathbf{4 . 2 0}$ \\
\hline
\end{tabular}


Factor 2 - $\quad$ Span of Service

Major loadings - $\quad$ SQ16 Earliest weekends

SQ17 Latest weekends

SQ15 Latest weekdays

SQ14 Earliest weekdays

Minor loadings - $\quad$ SQ5 Frequency of service

This construct relates to customer satisfaction with span of transit service availability.

The components are satisfaction with earliest weekday, latest weekday, earliest weekend day, and latest weekend day service. Frequency of service also slightly loaded on this factor.

\begin{tabular}{|lc|c|}
\hline \multicolumn{2}{|c|}{ Table 35-II: VOTRAN Factor 2 - Span of Service } \\
\cline { 2 - 3 } & \multicolumn{2}{c|}{ Scores } \\
\cline { 2 - 3 } Item & Index & Mean \\
\hline Latest weekdays & 112.45 & 3.49 \\
\hline Latest weekends & 114.72 & 3.56 \\
\hline Earliest weekends & 112.59 & 3.89 \\
\hline Earliest weekdays & 105.85 & 3.97 \\
\hline Frequency of Service & 109.54 & 3.79 \\
\hline \multirow{2}{*}{ Overall Mean } & & 3.85 \\
\hline
\end{tabular}




\section{Factor 3 - $\quad$ Perceptions of Safety}

Major loadings - $\quad$ SQ22 Safety at bus stop

SQ23 Safety on bus

SQ24 Safety getting off bus

SQ20 Clean buses and stop

\section{Minor loading - $\quad$ SQ26 Temperature of bus \\ SQ27 Availability of seats}

This construct relates to customer satisfaction with safety at bus stop, safety getting off bus, clean buses and stops, safety on bus, temperature on bus, and availability of seats. The temperature/availability of seats part of the construct may reflect some feeling of bus crowding as a safety issue - the more crowded the bus, the less safe the patrons feel. The connection between safety and cleanliness requires some explanation. One possibility is that the passenger construct relates to the apparent level of concern and respect the transit agency has for their passengers. Cleanliness is a very common way of showing respect and concern for customers - in restaurants, retail businesses, even in personal interactions. One of the correlates of transit agency concern for customers could be safety at bus stops, since the perception that the agency is taking care of its equipment and facilities may transfer over into perceptions of safety.

\begin{tabular}{|c|c|c|}
\hline \multirow[b]{2}{*}{ Item } & \multicolumn{2}{|c|}{ Scores } \\
\hline & Index & Mean \\
\hline Safety at Bus Stop & 110.53 & 4.33 \\
\hline Safety on Bus & 108.24 & 4.48 \\
\hline Safety Getting Off Bus & 108.57 & 4.41 \\
\hline Clean Buses and Stop & 113.25 & 4.27 \\
\hline Temperature of Bus & 116.04 & 4.46 \\
\hline Availability of Seats & 113.88 & 4.44 \\
\hline Overall Mean & & 4.40 \\
\hline
\end{tabular}


Factor 7 - $\quad \underline{\text { Schedule }}$

Major loadings - $\quad$ SQ12 Ease to obtain schedule

SQ13 Ease to use schedule

Minor loadings - $\quad$ SQ11 Value of fare/cost

The construct relates to customer experience using schedules. The main variables in this construct are satisfaction with the ease of using and obtaining the schedule. The loading of the value component indicates that, as in many other systems, making schedules easy to obtain and easy to use are primary elements in improving value perceptions.

\begin{tabular}{|c|c|c|}
\hline \multirow[t]{3}{*}{ Table 35-IV } & $7-\operatorname{Sch}$ & \\
\hline & \multicolumn{2}{|c|}{ Scores } \\
\hline & Index & Mean \\
\hline Ease to Obtain Schedule & 108.43 & 4.44 \\
\hline Ease to Use Schedule & 103.18 & 4.24 \\
\hline Value of Fare/Cost & 106.53 & 4.37 \\
\hline Overall Mean & & 4.35 \\
\hline
\end{tabular}




\section{Factor 9 - $\quad$ Experience of Bus Ride}

Major loadings - $\quad$ SQ29 Driver courtesy

SQ28 Ability to drive

SQ27 Availability of seats

Minor loadings - $\quad$ SQ26 Temperature in bus

This construct includes customer satisfaction with driver courtesy, ability to drive, and availability of seats. The variable temperature on bus also slightly loaded on this factor. The construct encompasses all the major experiential elements involved in the bus ride meeting the driver, finding a seat, the ride itself and the heat in the bus.

\begin{tabular}{|lc|c|}
\hline \multicolumn{2}{|c|}{ Table 35-V: VOTRAN Factor 9 - Experience of Bus Ride } \\
\cline { 2 - 3 } & \multicolumn{2}{c|}{ Scores } \\
\cline { 2 - 3 } & Index & Mean \\
\hline Driver Courtesy & 105.21 & 4.54 \\
\hline Ability to Drive & 107.72 & 4.62 \\
\hline Availability of Seats & 113.88 & 4.62 \\
\hline Temperature in Bus & 116.04 & 4.46 \\
\hline Overall Mean & & $\mathbf{4 . 5 1}$ \\
\hline
\end{tabular}


Factor 10 - $\underline{\text { Timeliness of Service }}$

Major loadings - $\quad$ SQ10 Time to make trip

SQ9 How regularly buses arrive on time

SQ5 Frequency of service

Minor loadings - $\quad$ SQ11 Value of fare/cost

SQ6 Ability to get where you want to go

This construct relates to issues of buses running frequently and on time, and the time to make trip. Also, convenience of routes slightly loaded on to this factor. All of these elements have an impact on customer value perception as well. These variables are connected with the customer's ability to arrive at their destination in a timely manner.

\begin{tabular}{|lc|c|}
\hline \multicolumn{2}{|c|}{ Table 35-VI: VOTRAN Factor 10 - Timeliness of Service } \\
\cline { 2 - 3 } & \multicolumn{2}{c|}{ Scores } \\
\cline { 2 - 3 } & Index & Mean \\
\hline Time to Make Trip & 106.36 & 3.96 \\
\hline How Regularly Buses Arrive On Time & 126.40 & 4.18 \\
\hline Frequency of Service & 109.54 & 3.79 \\
\hline Value of Fare/Cost & 106.53 & 4.37 \\
\hline Ability to Get Where You Want to Go & 111.18 & 4.35 \\
\hline Overall Mean & & $\mathbf{4 . 1 3}$ \\
\hline
\end{tabular}




\section{$\underline{\text { Customer Satisfaction Model }}$}

The following chart indicates which of VOTRAN's factors are the most important. The most influential factors are those with the largest importance values. For instance, since System Design, Perceptions of Safety, and Timeliness of Service have the highest importance values, VOTRAN should devote the most time and energy to these categories in order to improve customer satisfaction ratings. By looking at the individual items in each of these constructs, management can determine which items to focus on.

\section{Customer Satisfaction Model: VOTRAN}

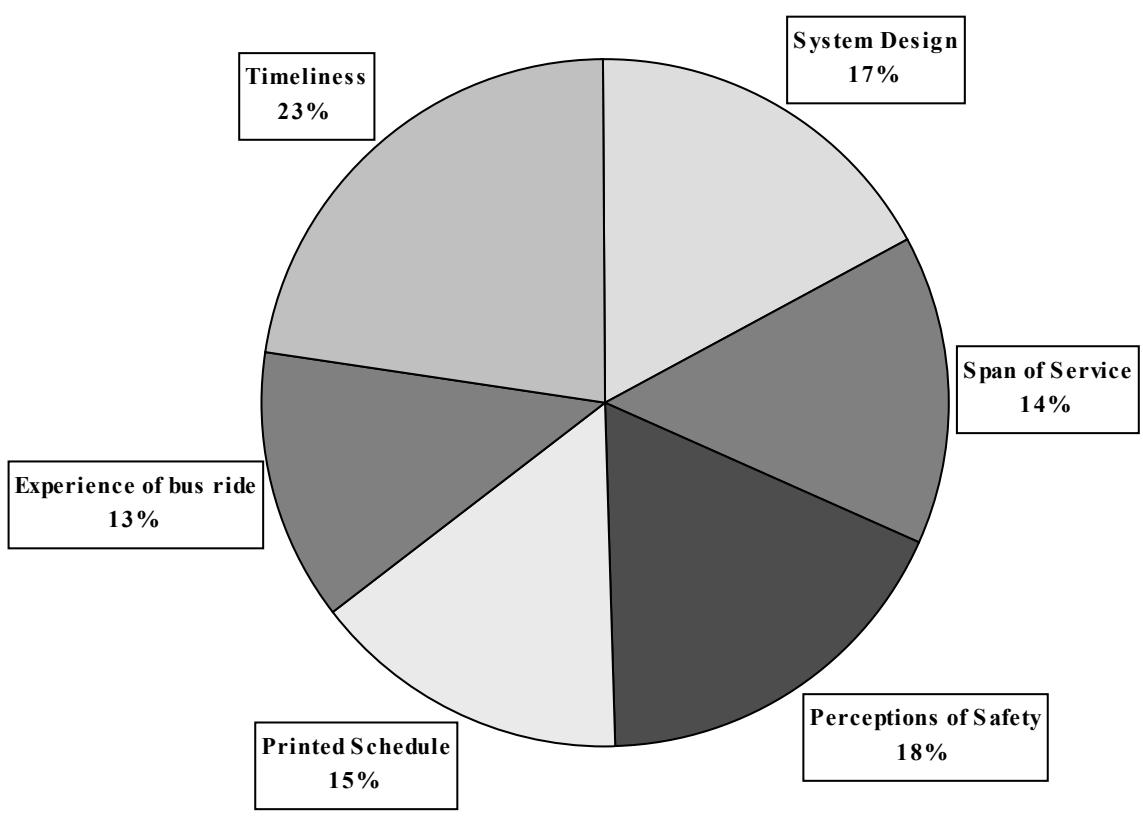

Figure 60: VOTRAN Customer Satisfaction Model 
Demographic Analysis

\section{Income}

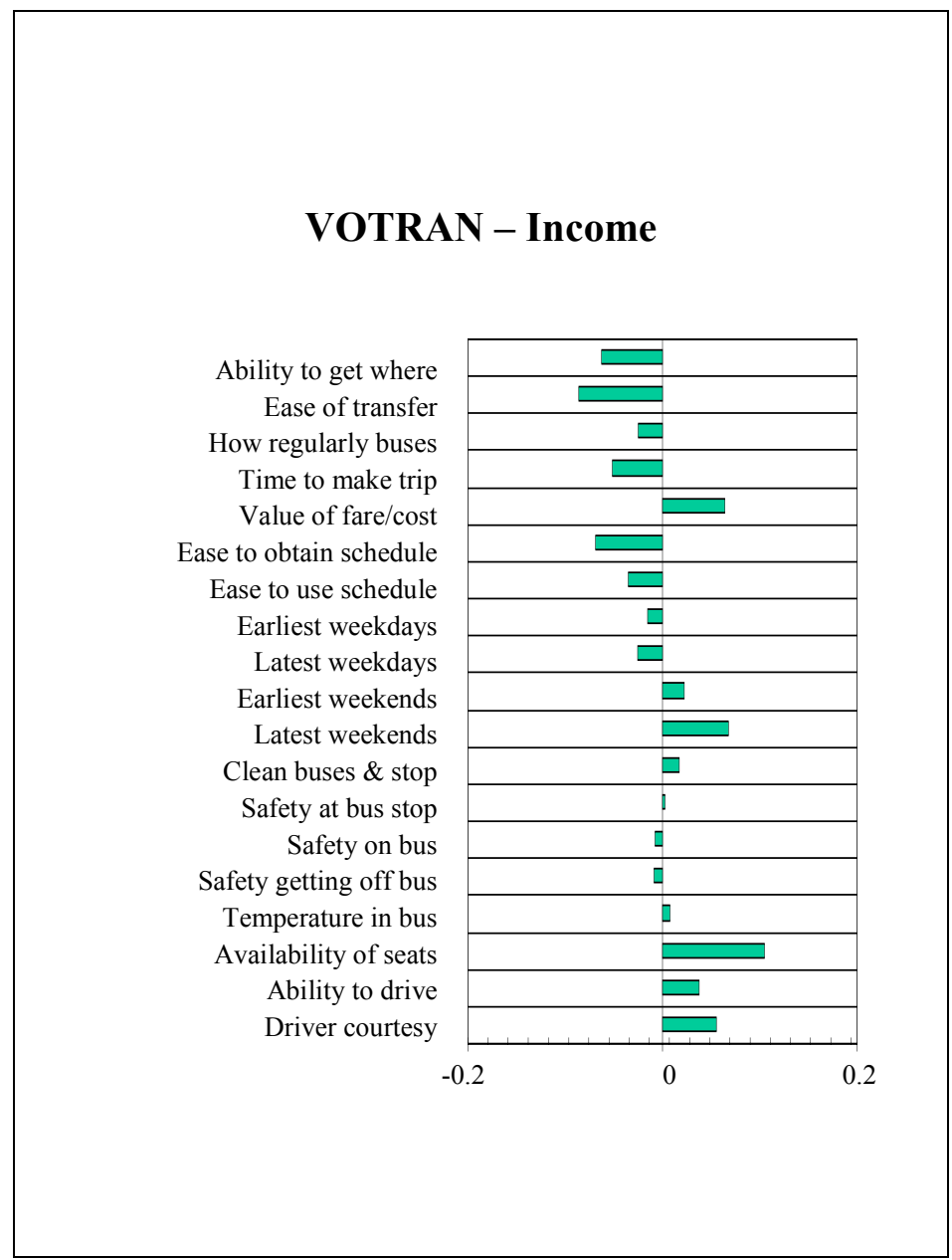

Figure 61: VOTRAN Correlation of income with satisfaction items

An analysis of non-linearly related items, frequency of service and number of transfers, revealed that riders with incomes over $\$ 50,000$ had lower levels of satisfaction with these items than any other group.

VOTRAN's lower income riders' experiences may be different than its higher income riders' experiences. Since lower income individuals usually ride transit more often, they tend to be more familiar with bus routes and schedules. The implication of this familiarity is that lower income individuals are inclined to be more satisfied with bus regularity, time to make trip, ease to obtain schedule, ease to use schedule, and ease of 
transfer. Lower income riders also probably use their familiarity of transit to set more realistic expectations for elements of the bus-riding experience than higher income riders. Thus the experiences that occur for which no prior expectation can reasonably be formed without prior use of the bus (such as on-time arrivals and ease of transfer) may be expected to get higher ratings among lower-income users. In contrast, higher income riders are more likely to be choice riders and hence will only use the bus when it is convenient for them. Thus those of higher income who are potential riders who feel that the bus is not convenient (in terms of routes, weekday span of service, etc.) will choose not to ride the bus and would therefore not be sampled. Lower income riders do not have this choice.

There are other mitigating factors to be considered. High-income riders are probably more satisfied with the temperature on the bus because the bus routes in the high-income neighborhoods may be less crowded. For similar reasons, satisfaction with availability of seats is higher among high-income riders. The 'ability to drive' rating may be higher due to better maintenance (i.e. fewer potholes) of the roads used on these routes. Assuming higher income individuals have access to other forms of transportation, they tend to be more satisfied with weekend service start and end times. Moreover, higher income individuals perceived comfort factors and value of fare to be more satisfactory. These high-income passengers rated seat availability, ability to drive, and driver courtesy higher than low-income riders. This probably relates to the fact that higher income individuals tend to ride buses in the nicer neighborhoods. Firstly, less passenger crowding causes seat availability to be rated higher. Secondly, roads that are located within high-income neighborhoods tend to be maintained better. Therefore, the 'ability to drive' rating may be higher due to better maintenance (i.e. fewer potholes) of the roads used on these routes. Moreover, drivers may be more relaxed and friendly when encountering a wellmaintained stretch of road. The result of high-income passengers being more satisfied with fare value/cost can be derived from a combination of circumstances (such as fare as a percentage of income and riding the bus only when it is convenient). 


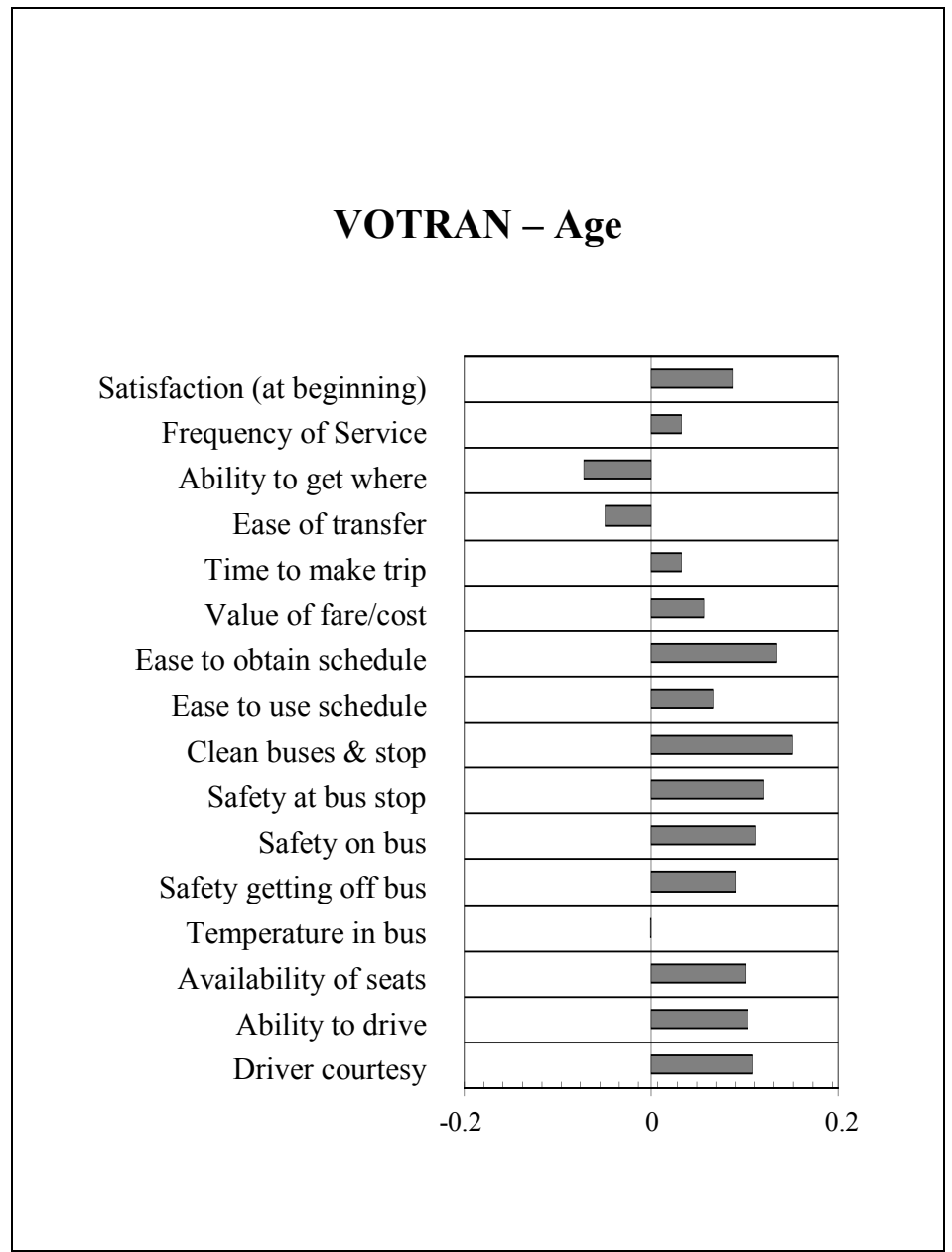

Figure 62: VOTRAN Correlation of age with satisfaction items

An analysis of non-linearly related items revealed that riders aged 45-59 had the lowest levels of satisfaction with number of transfers, and that riders aged 18-59 had the lowest levels of satisfaction with span of service.

Almost all of the satisfaction factors are positively correlated with age. Older respondents seem to be more satisfied with VOTRAN's transit service. As age increases, satisfaction increases for all but two categories, ability to get where and ease of transfer. Different experiences between the older and younger riders may cause the satisfaction differences between younger and older individuals. 
Individual time constraints and amount of leisure time can influence a passenger's satisfaction level. For instance, younger people are usually on a tighter schedule, such as arriving to work on time. Therefore, younger people will usually be more conscience of time delays. This can lead younger people to be less satisfied with frequency of service. Also, younger passengers usually have less leisure time. Assuming that they would like to gain additional leisure time, their dissatisfaction with time to make trip is understandable.

A combination of familiarity with surroundings as well as non-peak, short trips can lead to higher satisfaction ratings. Elderly people tend to utilize transit services in their particular neighborhood. Therefore, they are usually more familiar with their surroundings. Those who are more familiar with their surroundings tend to be generally more comfortable. Familiarity can lead older people to bestow higher ratings to driver courtesy, ability to drive, ease to obtain schedule, ease to use schedule, and safety factors. Riding a bus during non-peak trips increases the tendency of passengers to be more satisfied with the number of seats available on bus and cleanliness/comfort of bus. For example, a bus is perceived to be cleaner when there is less passenger congestion. Therefore, since older clients tend to ride the buses during non-peak service times, they tend to perceive the bus as being more clean/comfortable than younger passengers. Moreover, since younger people generally use the bus for longer trips, they will usually rate ease of transfer higher.

Finally, fare structure causes passengers to rate the value of transit differently. VOTRAN offers discount fares to senior citizens. Hence, it is not surprising that the value of trip satisfaction increases with age. 


\section{VOTRAN - Auto}

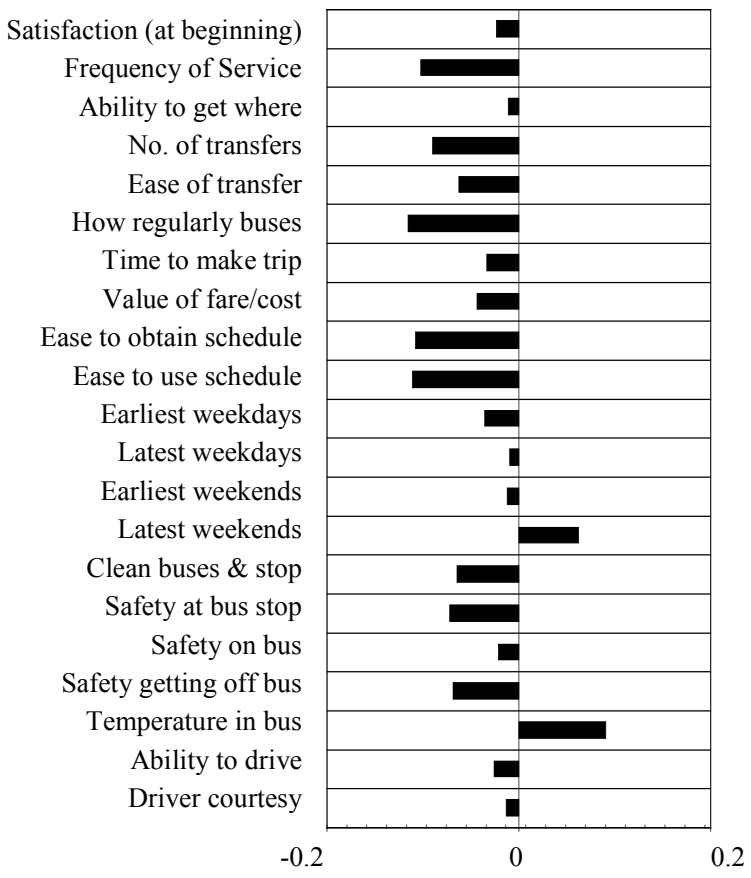

Figure 63: VOTRAN Correlation of auto ownership with satisfaction items

Auto ownership impacts these satisfaction levels because there is a difference between riding buses due to convenience versus riding them out of necessity. For instance, a positive correlation exists between number of automobiles and satisfaction with time service ends on weekends. Households that own automobiles have the luxury of 24-hour transportation. In contrast, households without vehicles are constrained by transit service times, taxi costs, and/or friends' schedules. Therefore, usually persons without automobiles are less satisfied with transit service begin and end times.

Satisfaction of the value of fare/cost is negatively correlated with number of automobiles in household. Those without cars perceive transit as more valuable because they utilize it for all types of trips. Without transit, these passengers would not be able to work, go to 
school, run errands, and/or utilize public facilities. In contrast, customers who own automobiles tend to underestimate the value of transit. Automobile owners do not know the difficulties associated with trying to obtain rides for errands when transit is not available.

Familiarity with transit service may also cause negative correlations between automobile ownership and transit satisfaction. For instance, ease to obtain schedule, ease to use schedule, ease of transfer, driver courtesy, dependability of buses and safety are all issues that are negatively correlated with ownership. Much can be explained by the fact that those who do not have cars use transit the most. Therefore, familiarity with bus schedules and bus routes will lead persons without cars to be more satisfied. A negative correlation between ease of transfer and ownership suggests that since the non-owners frequently depend upon bus transfers, they are used to the routine method and therefore perceive it as easier. Those who use transit often will also be more familiar with bus drivers and bus conditions, thereby inciting a higher satisfaction with driver courtesy and safety. Also, the riders who use transit the most will have a more realistic view of bus dependability and allow for more time to make trip.

Automobile owners may have extremely high expectations of transit service. For instance, owners tend to be less satisfied with the bus driver's ability to drive. A likely explanation is that automobile owners are used to driving and controlling a vehicle's whereabouts. Therefore, owners feel out of control when their rides depend on a complete stranger's driving abilities. Finally, lower income passengers perceived bus cleanliness and comfort to be more satisfactory than higher income passengers. This suggests that the riders who own automobiles are used to choosing their passengers. Auto owners may not be as comfortable with their fellow transit clientele and may be especially uncomfortable on crowded buses.

The positive correlation between number of automobiles in household and temperature in bus is difficult to explain. A cross-reference of low income/high auto households (across all systems, to allow sufficient sample size) shows that their level of satisfaction with temperature on the bus is marginally higher than those with low incomes and fewer vehicles (3.90 to 3.80). Those with high incomes and 3 or more autos have average ratings of 4.17. This finding may relate to the presence of vehicles with air conditioning in the household, i.e. that those who have vehicles with air conditioning find bus temperature comfortable, but perhaps others find it too cold. 
Transit riders who are black have higher ratings for VOTRAN service than those who are white, Hispanic, or of other ethnic origins. This is a very unusual finding, and indicates that service into predominantly black neighborhoods may be superior to service provided into other neighborhoods. 


\section{Ethnicity}

\begin{tabular}{|c|c|c|c|}
\hline & Table 36-I: VOTRAN Ethnicity & White & Black \\
\hline Sample size & & $\mathbf{9 5 9}$ & $\mathbf{6 4 8}$ \\
\hline & & & \\
\hline SQ08 & Ease of transfer & 4.21 & 4.53 \\
\hline SQ05 & Frequency of Service & 3.69 & 3.98 \\
\hline SQ15 & Latest weekdays & 3.42 & 3.68 \\
\hline SQ10 & Time to make trip & 3.90 & 4.15 \\
\hline SQ07 & No. of transfers & 3.83 & 4.08 \\
\hline SQ13 & Ease to use schedule & 4.19 & 4.41 \\
\hline SQ22 & Safety at bus stop & 4.28 & 4.47 \\
\hline SQ17 & Latest weekends & 3.49 & 3.69 \\
\hline
\end{tabular}

VOTRAN is unusual in that black riders have generally higher levels of satisfaction than whites. The elements of bus service for which blacks have higher levels of satisfaction include frequency of service, transfers, latest service, ease of using schedule, and time to make trip. This may be due to the level of service provided to neighborhoods that are predominantly populated by bus users of different ethnic origins. If neighborhoods with more blacks tend to use bus service more than neighborhoods that are predominantly white, it is likely that the bus service to those neighborhoods is more frequent and runs later and would be more likely to go directly to desired destinations (or require one transfer), and hence ratings would be higher.

\begin{tabular}{|l|c|c|c|}
\hline & Table 36-II: VOTRAN Ethnicity & White & Hispanic \\
\hline Sample size & & $\mathbf{9 5 9}$ & $\mathbf{8 3}$ \\
\hline & & & \\
\hline SQ17 & Latest weekends & 3.49 & 3.89 \\
\hline SQ22 & Safety at bus stop & 4.28 & 4.47 \\
\hline SQ10 & Time to make trip & 3.90 & 3.70 \\
\hline SQ05 & Frequency of Service & 3.69 & 3.48 \\
\hline SQ13 & Ease to use schedule & 4.19 & 3.94 \\
\hline SQ27 & Availability of seats & 4.46 & 4.17 \\
\hline SQ14 & Earliest weekdays & 3.96 & 3.66 \\
\hline
\end{tabular}


Hispanics have higher satisfaction levels than whites for latest weekend service and for safety. On several other items, including frequency, time to make trip, and earliest service, as well as availability of seats and ease of using schedule, whites have higher satisfaction levels. Whites are more likely to be choice riders, and may not ride at peak hours as much. However, the comfort level of whites regarding safety issues is a little lower, perhaps reflecting less experience using the bus.

\begin{tabular}{|l|c|c|c|}
\hline & Table 36-III: VOTRAN Ethnicity & Black & Hispanic \\
\hline Sample size & & $\mathbf{6 4 8}$ & $\mathbf{8 3}$ \\
\hline & & & \\
\hline SQ34 & Cleanliness/comfort (combined SQ 20 \& 21) & 4.22 & 4.48 \\
\hline SQ17 & Latest weekends & 3.69 & 3.89 \\
\hline SQ28 & Ability to drive & 4.72 & 4.51 \\
\hline SQ12 & Ease to obtain schedule & 4.53 & 4.32 \\
\hline SQ32 & Convenience of routes (combined SQ 6 \& 18) & 4.48 & 4.26 \\
\hline SQ31 & Satisfaction (combined) & 4.54 & 4.29 \\
\hline SQ27 & Availability of seats & 4.46 & 4.17 \\
\hline SQ07 & No. of transfers & 4.08 & 3.71 \\
\hline SQ08 & Ease of transfer & 4.53 & 4.14 \\
\hline SQ14 & Earliest weekdays & 4.08 & 3.66 \\
\hline SQ10 & Time to make trip & 4.15 & 3.70 \\
\hline SQ13 & Ease to use schedule & 4.41 & 3.94 \\
\hline SQ05 & Frequency of Service & 3.98 & 3.48 \\
\hline
\end{tabular}

Except for cleanliness/comfort and latest weekend service, blacks have uniformly higher satisfaction levels than Hispanics. Evidently VOTRAN is providing excellent service for black riders, as all of the ratings given are quite high. 


\begin{tabular}{|l|c|c|c|}
\hline & Table 36-IV: VOTRAN Ethnicity & White & Other \\
\hline Sample size & & $\mathbf{9 5 9}$ & $\mathbf{9 8}$ \\
\hline & & & \\
\hline SQ05 & Frequency of Service & 3.69 & 3.94 \\
\hline SQ07 & No. of transfers & 3.83 & 4.08 \\
\hline SQ33 & Dependability of buses (on time) (combined SQ 9 \& 19) & 4.16 & 3.94 \\
\hline SQ10 & Time to make trip & 3.90 & 3.63 \\
\hline SQ32 & Convenience of routes (combined SQ 6 \& 18) & 4.31 & 4.03 \\
\hline SQ16 & Earliest weekends & 3.82 & 3.52 \\
\hline SQ26 & Temperature in bus & 4.50 & 4.18 \\
\hline SQ14 & Earliest weekdays & 3.96 & 3.63 \\
\hline SQ34 & Cleanliness/comfort (combined SQ 20 \& 21) & 4.30 & 3.96 \\
\hline SQ24 & Safety getting off bus & 4.39 & 3.97 \\
\hline SQ22 & Safety at bus stop & 4.28 & 3.74 \\
\hline SQ15 & Latest weekdays & 3.42 & 2.88 \\
\hline SQ17 & Latest weekends & 3.49 & 2.93 \\
\hline SQ11 & Value of fare/cost & 4.39 & 3.76 \\
\hline
\end{tabular}

\begin{tabular}{|l|c|c|c|}
\hline & Table 36-V: VOTRAN Ethnicity & Black & Other \\
\hline Sample size & & $\mathbf{6 4 8}$ & $\mathbf{9 8}$ \\
\hline & & & \\
\hline SQ28 & Ability to drive & 4.72 & 4.53 \\
\hline SQ23 & Safety on bus & 4.53 & 4.31 \\
\hline SQ31 & Satisfaction (combined) & 4.54 & 4.29 \\
\hline SQ34 & Cleanliness/comfort (combined SQ 20 \& 21) & 4.22 & 3.96 \\
\hline SQ13 & Ease to use schedule & 4.41 & 4.14 \\
\hline SQ26 & Temperature in bus & 4.46 & 4.18 \\
\hline SQ33 & Dependability of buses (on time) (combined SQ 9 \& 19) & 4.22 & 3.94 \\
\hline SQ08 & Ease of transfer & 4.53 & 4.21 \\
\hline SQ14 & Earliest weekdays & 4.08 & 3.63 \\
\hline SQ32 & Convenience of routes (combined SQ 6 \& 18) & 4.48 & 4.03 \\
\hline SQ16 & Earliest weekends & 4.00 & 3.52 \\
\hline SQ24 & Safety getting off bus & 4.48 & 3.97 \\
\hline SQ10 & Time to make trip & 4.15 & 3.63 \\
\hline SQ11 & Value of fare/cost & 4.45 & 3.76 \\
\hline SQ22 & Safety at bus stop & 4.47 & 3.74 \\
\hline SQ17 & Latest weekends & 3.69 & 2.93 \\
\hline SQ15 & Latest weekdays & 3.68 & 2.88 \\
\hline
\end{tabular}


Members of 'Other' races, probably mainly Asians and native Americans, are significantly less satisfied with almost all elements of bus service than whites and blacks. This may be partially a cultural phenomenon (i.e., a tendency to give lower ratings on surveys) but may also reflect lower level of service (i.e., less frequency, and later start / earlier end times) to neighborhoods predominantly populated by these racial groups.

\begin{tabular}{|l|c|c|c|}
\hline & Table 36-VI: VOTRAN Ethnicity & Hispanic & Other \\
\hline Sample size & & $\mathbf{8 3}$ & $\mathbf{9 8}$ \\
\hline & & & \\
\hline SQ05 & Frequency of Service & 3.48 & 3.94 \\
\hline SQ07 & No. of transfers & 3.71 & 4.08 \\
\hline SQ27 & Availability of seats & 4.17 & 4.42 \\
\hline SQ13 & Ease to use schedule & 3.94 & 4.14 \\
\hline SQ33 & Dependability of buses (on time) (combined SQ 9 \& 19) & 4.16 & 3.94 \\
\hline SQ32 & Convenience of routes (combined SQ 6 \& 18) & 4.26 & 4.03 \\
\hline SQ26 & Temperature in bus & 4.42 & 4.18 \\
\hline SQ24 & Safety getting off bus & 4.43 & 3.97 \\
\hline SQ16 & Earliest weekends & 4.00 & 3.52 \\
\hline SQ34 & Cleanliness/comfort (combined SQ 20 \& 21) & 4.48 & 3.96 \\
\hline SQ11 & Value of fare/cost & 4.33 & 3.76 \\
\hline SQ15 & Latest weekdays & 3.60 & 2.88 \\
\hline SQ22 & Safety at bus stop & 4.47 & 3.74 \\
\hline SQ17 & Latest weekends & 3.89 & 2.93 \\
\hline
\end{tabular}

Hispanics are more satisfied than members of 'other' races for all elements except frequency, number of transfers, availability of seats, and ease of using schedule. Examination of service frequency and $\mathrm{O} / \mathrm{D}$ information appears to be in order for service to Hispanic neighborhoods. Also, the schedules may not be well understood by Hispanics.

As with most systems, however, satisfaction of members of 'other' races is lower on almost all elements of satisfaction, for reasons listed above. 
Gender

\begin{tabular}{|c|c|c|c|}
\hline & Table 37: VOTRAN Gender & Male & Female \\
\hline Sample size & & $\mathbf{7 6 2}$ & $\mathbf{9 5 5}$ \\
\hline & & & \\
\hline SQ33 & Dependability of buses (on time) (combined SQ 9 \& 19) & 3.99 & 4.32 \\
\hline SQ07 & No. of transfers & 3.74 & 4.06 \\
\hline SQ15 & Latest weekdays & 3.34 & 3.60 \\
\hline SQ16 & Earliest weekends & 3.76 & 3.99 \\
\hline SQ17 & Latest weekends & 3.44 & 3.63 \\
\hline SQ14 & Earliest weekdays & 3.87 & 4.06 \\
\hline
\end{tabular}

For span of services issues, as well as number of transfers required and dependability, women are more satisfied than men. Usage patterns of women may differ significantly, as they may be using transit more in midday periods and are less concerned about the ends of the schedule. They may also be using transit for shorter trips that are thus more likely to be provided with direct service rather than requiring transfers. 


\section{$\underline{\text { Recommendations }}$}

From the customer satisfaction model, it is possible to construct an "importanceperformance" matrix that graphically illustrates current bus riders' perceptions of VOTRAN operations.

\section{Importance / Performance Matrix Volusia County Riders' perceptions of VOTRAN service}

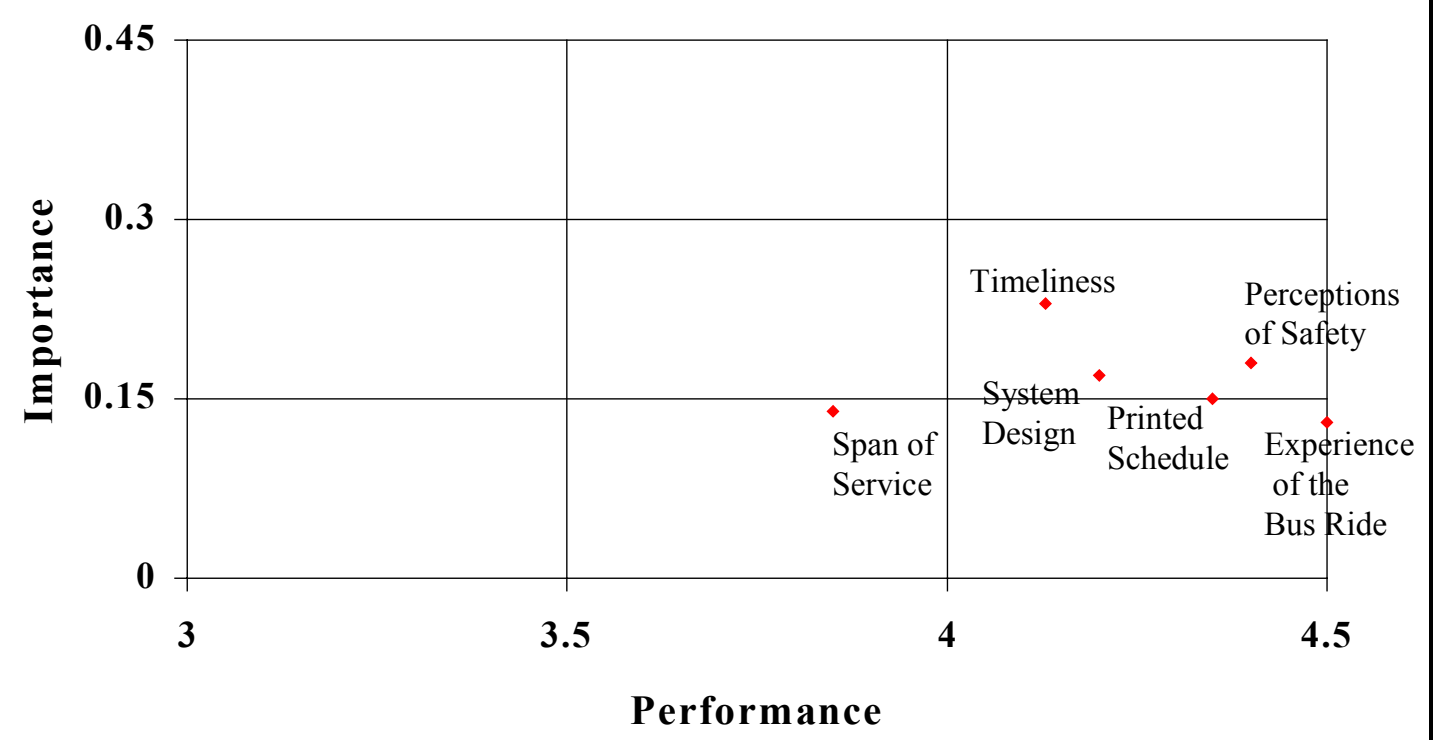

Figure 64: VOTRAN Importance/Performance Matrix

The chart has been divided into nine regions, reflecting various combinations of low, medium, and high performance and low, medium, and high importance. Borderline figures are interpreted as being in the higher of the importance categories they border on, but the lower of the performance categories. This provides the most conservative interpretation of the results. The interpretations of the chart regions are done as follows: 


\begin{tabular}{|c|c|c|c|}
\hline \multicolumn{4}{|c|}{$\begin{array}{c}\text { Table } 38 \\
\text { Interpretations of VOTRAN Chart Regions }\end{array}$} \\
\hline \multicolumn{2}{|c|}{ Chart region } & \multirow[b]{2}{*}{ Interpretation } & \multirow[b]{2}{*}{ Areas } \\
\hline Importance & Performance & & \\
\hline Low & High & Possibly reduce focus on this area & Experience of bus ride \\
\hline Low & Medium & Maintain performance - no action & Span of Service \\
\hline Low & Low & Maintain performance - no action & \\
\hline Medium & High & Maintain performance - no action & $\begin{array}{l}\text { System design, } \\
\text { Timeliness, Perceptions } \\
\text { of Safety, Printed } \\
\text { Schedule }\end{array}$ \\
\hline Medium & Medium & Maintain performance - no action & \\
\hline Medium & Low & Investigate for improvements & \\
\hline High & High & $\begin{array}{l}\text { Maintain performance - vigorous } \\
\text { quality checks, constant attention }\end{array}$ & \\
\hline High & Medium & Investigate for improvements & \\
\hline High & Low & Critical improvement area & \\
\hline
\end{tabular}

No factors fell into an area requiring immediate action.

According to the individual index and mean scores shown in Table I-J, VOTRAN is doing a good job with respect to the System Design factor category. All of the items indicate that VOTRAN's passengers perceive them better than average. VOTRAN scored exceptionally high index values for each of the items in this category: "number of transfer," "ease of transfer," and "ability to get where." However, VOTRAN's 
passengers seemed to score "number of transfers" lower than the rest of the items. Perhaps unproductive segments of the route schedule can be eliminated. Also, installing more bus shelters, benches, and other comfort factors at transfer locations will help to alleviate passengers' discomfort while waiting for transfers.

Table III-J reveals that VOTRAN's customers are satisfied with the Perceptions of Safety category, too. The overall mean was high. Also, compared to the other systems in Florida, VOTRAN's passengers are extremely satisfied with the items contained within this category: "safety at bus stop," "safety on bus," "clean buses and stop," "temperature of bus," "availability of seats" and "safety getting off bus." Since VOTRAN has been very successful at obtaining high safety perceptions from its clients, the only recommendation is to continue its exceptional service in safety related issues.

Finally, Table VI-J reveals another success. Compared to other systems within Florida, Timeliness of Service is regarded highly by VOTRAN's transit riders. In fact, all items within this category indicted very high index marks. The Timeliness of Service category contains five items: "time to make trip," "how regularly buses arrive on time," "frequency of service," "value of fare/cost," and "ability to get where you want to go." The only item that may need some reworking is "frequency of service." A few suggestions include adding buses to schedule, reevaluating routes, utilizing HOV lanes, adding express routes, et cetera.

Demographic correlations with satisfaction yielded few specific findings that could be translated into recommendations. Most of the findings were related either to choice use or familiarity, and did not provide a recommendation. 